\title{
WestVirginiaUniversity
}

THE RESEARCH REPOSITORY @ WVU

Graduate Theses, Dissertations, and Problem Reports

2004

\section{Development of coker feeds from aromatic oil and bituminous coal digests}

\author{
L. Mitchell Clendenin \\ West Virginia University
}

Follow this and additional works at: https://researchrepository.wvu.edu/etd

\section{Recommended Citation}

Clendenin, L. Mitchell, "Development of coker feeds from aromatic oil and bituminous coal digests" (2004). Graduate Theses, Dissertations, and Problem Reports. 1750.

https://researchrepository.wvu.edu/etd/1750

This Thesis is protected by copyright and/or related rights. It has been brought to you by the The Research Repository @ WVU with permission from the rights-holder(s). You are free to use this Thesis in any way that is permitted by the copyright and related rights legislation that applies to your use. For other uses you must obtain permission from the rights-holder(s) directly, unless additional rights are indicated by a Creative Commons license in the record and/ or on the work itself. This Thesis has been accepted for inclusion in WVU Graduate Theses, Dissertations, and Problem Reports collection by an authorized administrator of The Research Repository @ WVU. For more information, please contact researchrepository@mail.wvu.edu. 


\title{
Development of Coker Feeds from Aromatic Oil and Bituminous Coal Digests
}

\author{
L. Mitchell Clendenin \\ Thesis submitted to the \\ College of Engineering and Mineral Resources \\ at West Virginia University \\ in partial fulfillment of the requirements \\ for the degree of

\section{Master of Science \\ in} \\ Chemical Engineering
}

\author{
Peter G. Stansberry, Ph.D., Chair \\ Alfred H. Stiller, Ph.D. \\ John W. Zondlo, Ph.D. \\ Charter D. Stinespring, Ph.D. \\ Department of Chemical Engineering \\ Morgantown, West Virginia \\ 2004
}

Keywords: Coal, solvent, hydrogenation, digestion, coke, air-blowing 


\title{
ABSTRACT \\ Development of Coker Feeds from Aromatic Oil and Bituminous Coal Digests
}

\author{
L. Mitchell Clendenin
}

Kingwood coal has been digested with two coal derived (anthracene oil and carbon black base) and two petroleum derived (slurry oil and Maraflex oil) aromatic oils, both raw and catalytically hydrogenated. After a parametric study to determine reaction conditions ( $\mathrm{T}, \mathrm{P}$, solvent, hydrogenation level), six one-gallon digestions were performed, using hydrogenated carbon black base (HCBB-L3), hydrogenated slurry oil (HSO-L3), hydrogenated Maraflex oil (HMO-L3) and combinations thereof. After solids separation, the coal digest liquids were air-blown at $250^{\circ} \mathrm{C}$ for 5 hours. Air-blown digests were carbonized and all products analyzed physically and chemically to determine the feasibility of using this process in producing anode coke feeds. Though solvent digestions proved successful in coal conversion, particularly using HCBB-L3 at $425^{\circ} \mathrm{C}$, which achieved $93.89 \%$ (daf) coal conversion, the air-blown digests do not form anisotropic cokes upon carbonization. However, vacuum distillation of the digests results in material that becomes anisotropic upon carbonization. 


\section{ACKNOWLEDGEMENTS}

This research is made possible through grant \# CE-FC26-02NT41596 from the Department of Energy and the National Energy Technology Laboratories. The project title is "Production of Carbon Products Using a Coal-Extraction Process".

The author would like first to thank his research advisor, Dr. Peter G. Stansberry, for his encouragement, guidance and support. This would not be possible without his hard work over many years in the WVU Carbon Products Group. Additionally, many thanks to the other committee members, Dr. Al Stiller, Dr. Charter Stinespring and Dr. John Zondlo, for their support and advice. Special thanks to Dr. John Zondlo, whose advice and assistance made returning to graduate school possible. Also, the contribution of co-workers and other graduate students is noted, especially Nathan King, Jennie Wheeler, Morgan Summers, Jason Hissam, Jimmy Rhoades, Mike Velez, Liviu Magean and Elliot Kennel.

The author also wishes to thank his parents, Larry and Judy Clendenin, his mother-in-law, Elaine Cawthon, and his extended family whose support has made this experience less difficult to get through. Thanks also to the many friends whose support and encouragement has been invaluable.

The author would like to give thanks and praise to his Lord and Savior, Jesus Christ, with whom all things are possible.

Lastly, the author wishes to give a special note of thanks to his wife, Sherry, for her sacrifice and hard work during this time, without which this would not have been possible. I could not possibly have made it through this without her love and encouragement, and God willing, these first few years of sacrifice as a married couple will provide us with the opportunity to pursue our dreams in the future 


\section{TABLE OF CONTENTS}

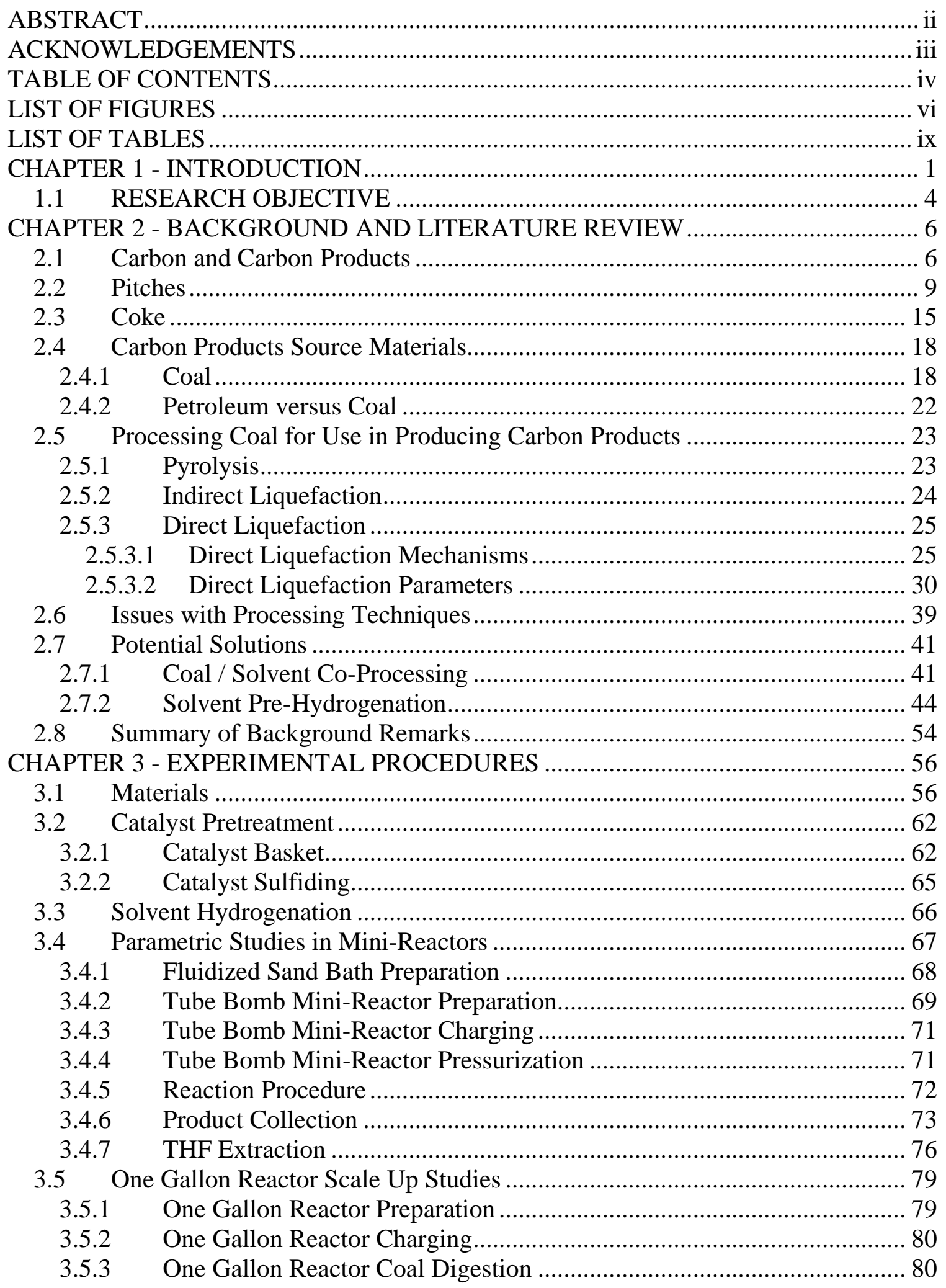


3.5.4 One Gallon Reactor Products Collection.............................................. 81

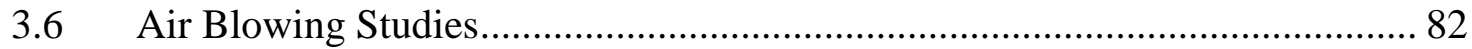

3.6.1 Air Blowing Reactor Preparation........................................................... 82

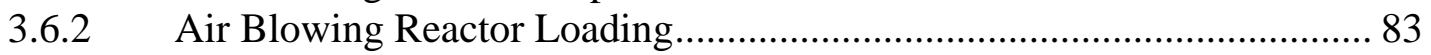

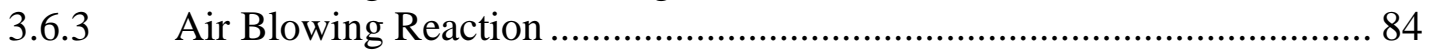

3.6.4 Air Blowing Reactor Product Recovery ........................................... 84

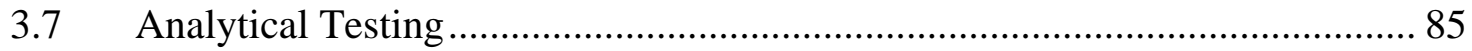

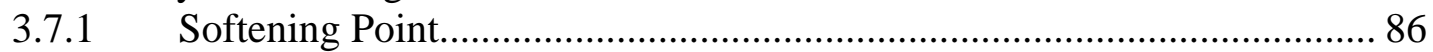

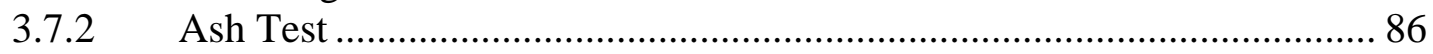

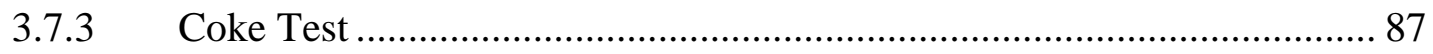

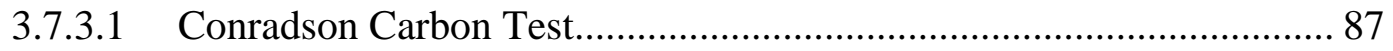

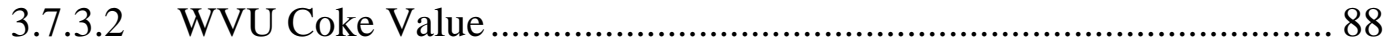

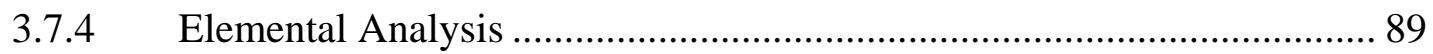

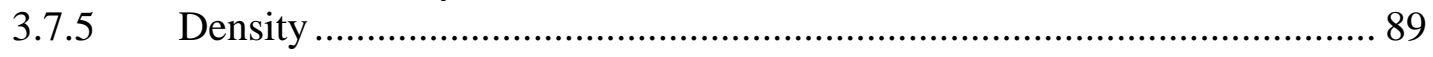

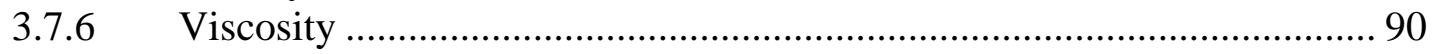

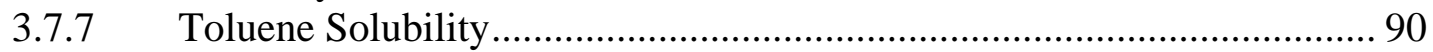

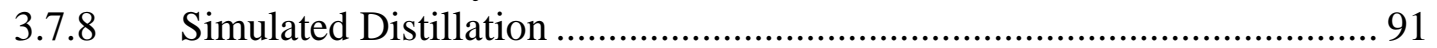

3.7.9 Fourier-Transform Infrared Spectroscopy (FTIR) ............................... 93

Experimental Error............................................................................... 94

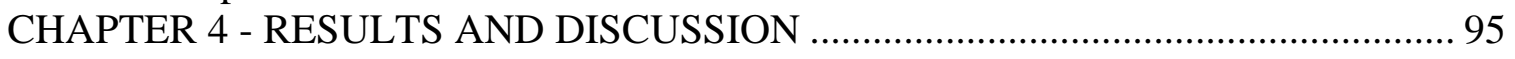

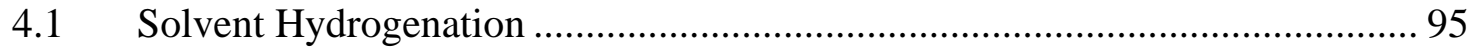

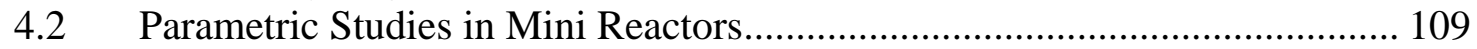

4.3 One Gallon Scale-Up Studies ................................................................ 114

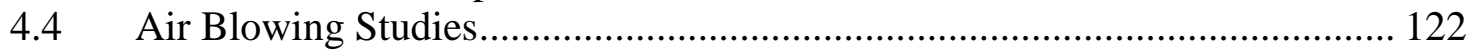

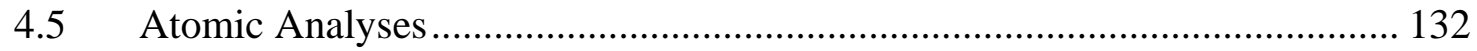

4.6 Vacuum Distillation of Coal Digest Liquids ............................................. 139

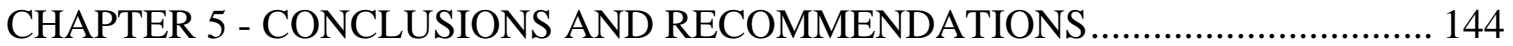

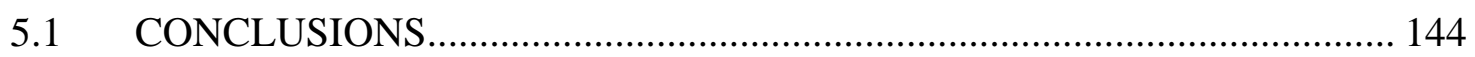

5.2 RECOMMENDATIONS FOR FUTURE WORK ................................... 147

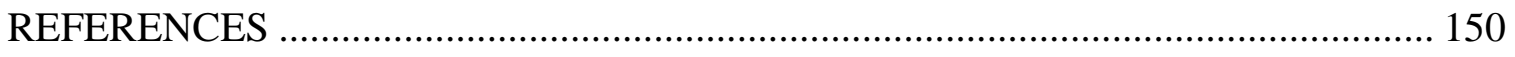

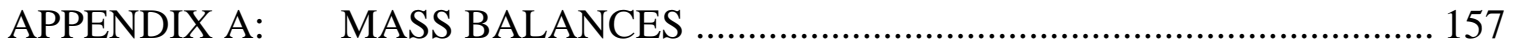

APPENDIX B: $\quad$ ANALYTICAL DATA TABLES............................................... 163

APPENDIX C: $\quad$ MATERIAL SAFETY DATA SHEETS ....................................... 185 


\section{LIST OF FIGURES}

Figure 2.1: Optical Textures of Isotropic and Anisotropic Cokes ...........................8

Figure 2.2: Softening Point of coal tar, hydrogenated coal tar, and petroleum

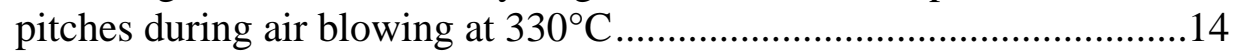

Figure 2.3: One Theoretical Molecular Unit of Coal...........................................21

Figure 2.4: Proposed Free Radical Mechanism of Liquefaction .............................26

Figure 2.5: Solvent Mediated Hydrogenolysis of a Strong Linkage in Coal............27

Figure 2.6: Conceptual Reaction Sequences in Coal Liquefaction..........................29

Figure 2.7: Effect of Carbon Content on Liquid Product Yield ...............................33

Figure 2.8: Dependence of solvation yield from a sub-bituminous coal on the reactive maceral content ....................................................................34

Figure 2.9: Wyodack Coal Conversion vs. Ash Content .......................................38

Figure 2.10: Schematic flow diagram of the NEDOL 150 ton/day pilot plant.........47

Figure 2.11: Variation of naphthenic $\mathrm{H}_{2}$ contents in feed and hydrogenated solvent as a function of $\mathrm{H}_{2}$ gas consumption in solvent treatment.......48

Figure 2.12: Influence of Solvent Pretreatment with Fresh Anthracene Oil and Hydrogenated Anthracene Oil..................................................50

Figure 2.13: Conversion as a function of $\mathrm{H}$ n.m.r. aromaticity of Hydrogenated solvents using $\mathrm{H}_{2}$ gas and $\mathrm{N}_{2}$ gas atmospheres ................................54

Figure 3.1: Process Flow Diagram for Coal Digestion (small scale) ......................60

Figure 3.2: Process Flow Diagram for Coal Digestion (1-gallon scale)..................61

Figure 3.3: Catalyst Basket Design....................................................................64

Figure 3.4: Completed and Loaded Catalyst Basket..............................................64

Figure 3.5: Diagram of the Tube Bomb Mini-Reactors.........................................70

Figure 3.6: Product Removal Thimble / Flask Apparatus .......................................75

Figure 3.7: Soxhlet Condenser Apparatus ...........................................................78

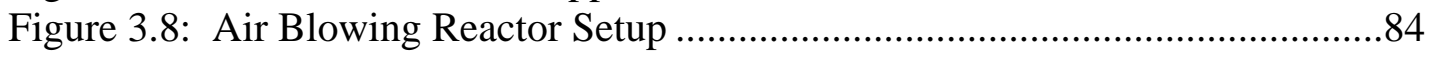

Figure 4.1: Temperature and Pressure for HCBB Level 1 Hydrogenation................96

Figure 4.2: Temperature and Pressure for HCBB Level 2 Hydrogenation...............97

Figure 4.3: Temperature and Pressure for HCBB Level 3 Hydrogenation...............97

Figure 4.4: Temperature and Pressure for HSO Level 3 Hydrogenation .................98

Figure 4.5: Temperature and Pressure for HMO Level 3 Hydrogenation .................98

Figure 4.6: Boiling Point Curves for Raw Solvents ............................................100

Figure 4.7: Boiling Point Curves for CBB Solvents.............................................101

Figure 4.8: Boiling Point Curves for Slurry Oil Solvents........................................101

Figure 4.9: Boiling Point Curves for Maraflex Oil Solvents .....................................102

Figure 4.10: First Derivative of Boiling Point Curve for CBB versus

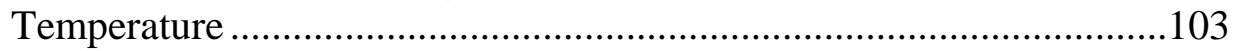

Figure 4.11: First Derivative of Boiling Point Curve for HCBB-L1 versus Temperature ................................................................................104

Figure 4.12: First Derivative of Boiling Point Curve for HCBB-L2 versus Temperature ...............................................................................104

Figure 4.13: First Derivative of Boiling Point Curve for HCBB-L3 versus

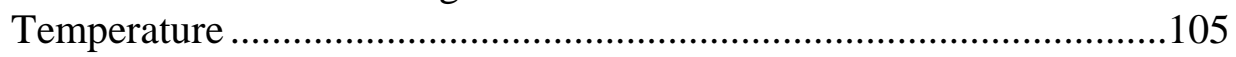


Figure 4.14: First Derivative of Boiling Point Curve for Slurry Oil versus

Temperature 105

Figure 4.15: First Derivative of Boiling Point Curve for HSO-L3 versus

Temperature. 106

Figure 4.16: First Derivative of Boiling Point Curve for Maraflex Oil versus Temperature. 106

Figure 4.17: First Derivative of Boiling Point Curve for HMO-L3 versus Temperature. 107

Figure 4.18: Amount of Sulfur and Nitrogen (by wt \%) in Solvents........................109

Figure 4.19: Conversion versus Temperature at $\mathrm{P}=0$ psig $\mathrm{N}_{2} \ldots \ldots \ldots \ldots \ldots \ldots \ldots \ldots \ldots \ldots . . . . . . . . . . .110$

Figure 4.20: Conversion versus Temperature at $\mathrm{P}=500$ psig $\mathrm{N}_{2} \ldots \ldots \ldots \ldots \ldots \ldots \ldots \ldots . . . . . . .110$

Figure 4.21: Coal Conversion for Raw Solvents at $\mathrm{T}=400^{\circ} \mathrm{C}$

Figure 4.22: Conversion versus Hydrogenation Level at $\mathrm{T}=400^{\circ} \mathrm{C}$......................113

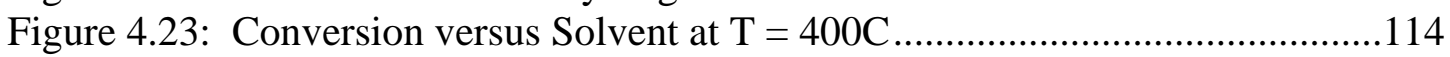

Figure 4.24: Scale-Up Reaction Conversion versus Solvent Choice........................117

Figure 4.25: Solvent C/H Atomic Ratio versus Digestion Conversion (daf) ............119

Figure 4.26: Solvent Aromaticity versus Digestion Conversion (daf) .....................120

Figure 4.27: Solvent $\mathrm{H}_{\mathrm{ar}} / \mathrm{H}_{\mathrm{al}}$ Ratios versus Digestion Conversion (daf)...................120

Figure 4.28: Solvent $(\mathrm{C}=\mathrm{C})_{\mathrm{ar}} /\left(\mathrm{H}_{\mathrm{ar}}+\mathrm{H}_{\mathrm{al}}\right)$ Ratios versus Digestion Conversion (daf) 121

Figure 4.29: Solvent $(\mathrm{C}=\mathrm{C})_{\mathrm{ar}} / \mathrm{H}_{\mathrm{ar}}$ Ratios versus Digestion Conversion (daf) ............121

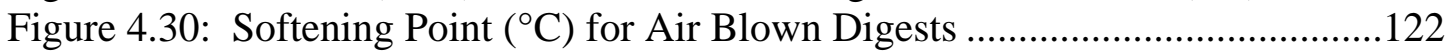

Figure 4.31: Ash Content in Coal Digest Liquids and Air Blown Digests................124

Figure 4.32: Conradson Carbon Coke Yield of Coal Digests..................................125

Figure 4.33: WVU Coke Yield of Coal Digests ...................................................125

Figure 4.34: Toluene Insolubles of Coal Digests...................................................126

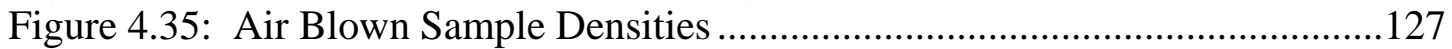

Figure 4.36: Viscosity versus Temperature $\left({ }^{\circ} \mathrm{C}\right)$ for Coal Digests and Air

Blown Coal Digests: A) A086, B) A090, C) A095, D) A098,

E) A100, F) B003

Figure 4.37: Photomicrographs (160X) of green cokes made from A090 Coal

Digest and Air Blown A090 Coal Digest ...........................................130

Figure 4.38: Photomicrographs (160X) of green cokes made from A095 Coal

Digest and Air Blown A095 Coal Digest .........................................130

Figure 4.39: Photomicrographs (160X) of green cokes made from A098 Coal

Digest and Air Blown A098 Coal Digest ..........................................131

Figure 4.40: Photomicrographs (160X) of green cokes made from A100 Coal Digest and Air Blown A100 Coal Digest .131

Figure 4.41: Photomicrographs (160X) of green cokes made from B003 Coal

Digest and Air Blown B003 Coal Digest..........................................131

Figure 4.42: Sulfur Concentrations in Digest Processing .......................................133

Figure 4.43: Nitrogen Concentration in Digest Processing .....................................134

Figure 4.44: Hydrogen Concentration in Digest Processing ..................................135

Figure 4.45: Carbon Concentration in Digest Processing........................................135

Figure 4.46: Solvent Atomic C/H Ratio versus Air-Blown Softening Point $\left({ }^{\circ} \mathrm{C}\right)$....136

Figure 4.47: Solvent Aromaticity versus Air-Blown Softening Point $\left({ }^{\circ} \mathrm{C}\right)$...............137 
Figure 4.48: Coal Digest Atomic C/H Ratio versus Air-Blown Softening

Point $\left({ }^{\circ} \mathrm{C}\right)$

Figure 4.49: Coal Digest Aromaticity versus Air-Blown Softening Point $\left({ }^{\circ} \mathrm{C}\right)$........138

Figure 4.50: Softening Point for Air Blown and Vacuum Distilled Digests $\left({ }^{\circ} \mathrm{C}\right)$....141

Figure 4.51: WVU Coke Yields for Air-Blown and Vacuum Distilled Digests .......141

Figure 4.52: Photomicrographs (160X) of green cokes made from Air Blown A090 Coal Digest and Vacuum Distilled A090 Coal Digest... 142

Figure 4.53: Photomicrographs (160X) of green cokes made from Air Blown A095 Coal Digest and Vacuum Distilled A095 Coal Digest.

Figure 4.54: Photomicrographs (160X) of green cokes made from Air Blown A098 Coal Digest and Vacuum Distilled A098 Coal Digest..... 142

Figure 4.55: Photomicrographs (160X) of green cokes made from Air Blown A100 Coal Digest and Vacuum Distilled A100 Coal Digest.....

Figure 4.56: Photomicrographs (160X) of green cokes made from Air Blown B003 Coal Digest and Vacuum Distilled B003 Coal Digest 


\section{LIST OF TABLES}

Table 2.1: Characterizations of Various Commercial Pitches .................................11

Table 2.2: ASTM Std. D 388-66 System for Coal Rank Classification ....................20

Table 2.3: Percent Elemental Composition of Various Coal Ranks ..........................22

Table 2.4: Definition of Primary Liquids from Berkowitz ...................................29

Table 2.5: Fundamental Properties Important in Coal Liquefaction .........................31

Table 2.6: Total Conversion for Illinois No.6 coal and Oil Solvents at $430^{\circ} \mathrm{C}$ in Tubing Reactors for 1 hour under 1000 psig $\mathrm{H}_{2}$ (cold) ........................43

Table 2.7: Properties of Heavy Solvents..............................................................49

Table 2.8: Effect of Using Hydrogenation Catalysts in Preparing HVPTO .............52

Table 3.1: Characteristics of Kingwood Coal.........................................................59

Table 3.2: Elemental Analysis Results for Coal and Four Candidate Solvents........59

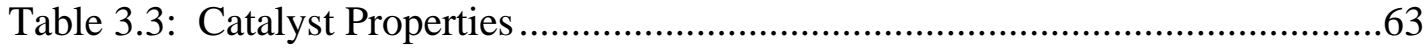

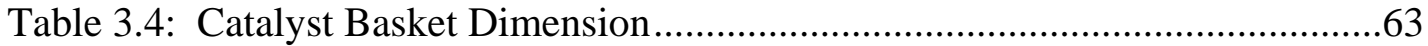

Table 3.5: Solvent Hydrogenation Reaction Conditions .....................................67

Table 3.6: One Gallon Reactor Dimensions ..........................................................80

Table 4.1: Solvent Hydrogenation Results ............................................................99

Table 4.2: Elemental and FTIR Analysis Data for Solvents..................................108

Table 4.3: One-Gallon Scale-Up Digestion Details..............................................115

Table 4.4: Atomic C/H Ratios for Coal Liquid Digests and Feed Coal ...................118

Table 4.5: Details of Vacuum Distillation of Coal Digests ....................................140 


\section{CHAPTER 1 - INTRODUCTION}

Carbon in one form or another has been used throughout the history of mankind. From wood used to make fire for heat in prehistoric times to polymers and pharmaceuticals made from petroleum fractions, carbon has been an integral part of human existence. Carbon is the very basis of an entire division of science, organic chemistry, and in fact, human beings are made up in part of carbon. However, the carbon products industry has been going through a major period of change and adaptation over the past few years, particularly with respect to source materials.

The most common uses of carbonaceous materials are as fuels, specifically, from the natural gas, petroleum and coal deposits that are scattered throughout the world. Petroleum, in addition to being the chief source of automobile fuel, is the major source of carbon used in products such as petrochemicals, coke, asphalt and road tar, etc. ${ }^{(1)}$. However, there are some problems associated with all of these fossil fuels. Traditional coal use, combustion, is seen as environmentally unfavorable, and the US petroleum reserves are declining in both quantity and quality. This is due to the rising concentrations of impurities like sulfur, vanadium, nickel, and other mineral matter, and this condition is only expected to worsen.

Many of the carbon products currently produced from petroleum could be produced from coal, which is both a readily available and commercially inexpensive domestic feed material. In non-fuel uses, coal is currently processed in some areas to produce metallurgical coke, granular activated carbons, coal tars, and pitches. The products are all either a direct result or a by-product of the metallurgical coke process, in which volatile matter is evolved during the coking process, and then is collected and 
condensed into coal tar. The coal tar is then distilled to produce both lighter products (tar acids/bases, creosote oils, naphthalenes, etc.) and a heavy product called coal tar pitch. Pitch can be made, not only as a by-product of the metallurgical coking process, but also as a petroleum derivative, or through the solvent extraction of coal.

Coal-tar pitch is used as a binder in the production of carbon electrodes for electric furnaces and for anodes used by the aluminum industry. Other pitches are used as impregnants to enhance the density and the strength of manufactured carbon, while others can be made into nuclear grade graphite or even carbon fibers and foams. In some instances, pitches are converted into cokes for electrode applications.

Anodes for the aluminum industry are generally made from delayed petroleum coke (about $60-70 \%$ as filler), coal-tar pitch (10-15\%, as a binder) and anode butts (spent anodes used as recycle material). This petroleum coke is a by-product in petroleum refining, and often only a small portion of the coke made in the delayed coking process is suitable for use as anode coke ${ }^{(2)}$. Graphite electrodes, on the other hand, are made from selected cokes (either petroleum or coal-tar based), which are crushed and milled, then mixed with a coal-tar binder pitch and then extruded into the final rod shapes which are then baked, first to $800^{\circ} \mathrm{C}$ and then to $3000^{\circ} \mathrm{C}$, to form crystalline graphite ${ }^{(3)}$. In both of these cases, the quality of the coke fillers (specifically the petroleum-derived cokes) is decreasing rapidly.

An alternate method of producing the coke precursors would help in the growth of this industry. One method of producing a coke material is the liquefaction of coal. This can be done indirectly, which produces $\mathrm{CO}$ and $\mathrm{H}_{2}$ gas (that can then be reformed into a variety of hydrocarbon materials) and a by-product tar. In direct liquefaction, on the 
other hand, the coal is thermally treated in a solvent to produce lower molecular weight organic species. This can be done using a hydrogen donor solvent.

A common direct liquefaction solvent used extensively in research is tetralin (1,2,3,4-tetrahydronaphthalene). It is a very effective liquefaction solvent, but it is very expensive. Also, most of the tetralin is converted to naphthalene during the liquefaction process. Thus, any attempt at recycling the solvent will require additional, typically costly, re-hydrogenation steps. Even if recycle was not economically attractive, the spent solvent must still be removed, which is itself costly. At West Virginia University, NMP (N-methyl-2-pyrrolidone) has been used as an extraction solvent to remove unconverted coal and mineral matter from the liquefied coal. NMP is also an expensive solvent, and use of it introduces additional solvent removal and purification steps. Much work has been performed, both at West Virginia University and elsewhere, on using industrial byproduct liquids (such as pyrolyzed tire oil, anthracene oil, petroleum residues, even creosote oils) as the liquefaction medium. While some success in this research has been made, there remains the need for solvent recovery. Also, in most direct liquefaction studies, the desired product is liquid fuel, and thus hydrogen gas must be used in significant quantities to accomplish the desired conversions.

To bypass the use of expensive process solvents like tetralin and NMP, to eliminate the expensive process steps solvent recovery and re-hydrogenation, and to help lessen the costly use of high-pressure hydrogen gas, it is proposed that coal and an industrial by-product solvent can be "co-processed" into a pitch-like feed suitable for coke making. Here, there is no recovery or recycle, as the solvent is incorporated into the final product. This material can then be further treated so that the resultant product 
would have the desired characteristics of a coke feed, and be relatively free of mineral impurities that degrade carbon products made from traditionally produced coke feeds.

This study is very timely, as the concerns in the aluminum industry with regards to the quality of the coke used in the production of aluminum metal are growing. As the coke quality decreases, the amount of coke required in producing the same amount of aluminum metal increases and the amount of $\mathrm{CO}_{2}$ evolved in the aluminum production increases. Thus, the production of a high quality coker feed material is of vital importance.

\subsection{RESEARCH OBJECTIVE}

In this thesis study, the objective is to develop and characterize coal and heavy oil digests as coker feeds, specifically for use in carbon anodes, as well as investigate the effectiveness of solvent pre-hydrogenation and air-blowing as a method of digest modification. Kingwood coal was digested with a variety of industrial by-product aromatic oils, both raw (as received) and treated (hydrogenated to varying degrees). Carbon Black Base (a creosote oil) was received from Koppers Industries. Reilly Industries donated a heavy oil (anthracene oil). Both materials are derived from the metallurgical coking process. Also received were two petroleum derived solvents, RCC slurry oil and Maraflex oil, both from Marathon-Ashland Petroleum, LLC. Most of these solvents were hydrogenated to varying degrees over a supported Ni-Mo catalyst. The solvents were processed with coal in a parametric study using tubing bomb reactors, and the products extracted using tetrahydrofuran (THF). The optimal digestion conditions are determined from the results of this parametric study. The THF-insoluble portion of the 
products was used to calculate the coal conversion, and the results of this parametric study were then used to design scaled-up coal digestions in a larger reactor (1-gal). This scale-up study would not only test the digestion on a larger scale, but provide large amounts of material for use in additional process studies. Finally, these digests were subjected to air-blowing treatments to modify the physical and chemical properties, and then carbonized. The air-blown digests and carbonized air-blown digests were analyzed to determine if they would be acceptable feed materials for anode cokes. Air blowing was chosen as the method of treatment in this thesis study because of its ease of application and effectiveness, as reported by N. King in his thesis study at West Virginia University ${ }^{(4)}$. 


\section{CHAPTER 2 - BACKGROUND AND LITERATURE REVIEW}

\subsection{Carbon and Carbon Products}

Carbon, the sixth element on the periodic table, is perhaps the most special element. There are other elements that have singular characteristics and are even more valued and precious than carbon, but carbon stands alone. Perhaps the chief reason for this is the ability of carbon to bond, not only to elements like oxygen and hydrogen, but also to itself. These carbon-carbon bonds are at the heart of the study of organic chemistry. A compound with two or three of these carbon-carbon bonds may exist as a vapor, such as ethylene or propane, while a string of carbons several hundred long, even several thousand long, make up a polymer, like polyethylene and nylon 66. Carbon is present in a wide variety of forms naturally and, as technology has advanced, an increasing number of man-made, synthetic forms.

Carbon in its natural elemental form is available in three different allotropic structures ${ }^{(3)}$. Diamond, a tetrahedrally bonded crystal, is a result of thousands of years of heat and pressure and is sought after the world over as a precious stone. Carbon can also appear as a soccer-ball-shaped form known as fullerenes. Finally, graphite, which is the most abundant naturally occurring form of elemental carbon, is a crystalline material where the carbon atoms are arranged in large planar sheets. These planes are held together by van der Waal's forces. Graphite has a number of desirable qualities that make it a valuable commodity. Properties such as the thermal conductivity, electrical conductivity, hardness, and porosity result from the crystal structure of the material. 
Most carbon products are made based on the graphite structure and control of the crystal structure is of paramount importance to industry.

A material that is highly graphitic, or in other words, highly ordered structurally on the microscopic level, is referred to as an anisotropic material. Graphite can be ground to a fine enough powder to eliminate essentially the bulk anisotropy, but this material would still be anisotropic on the microscopic scale. The opposite of anisotropic, naturally, is isotropic, which is used to describe a material that contains no real longrange order in the microstructure. This property of carbonaceous materials can usually be detected using reflected-light optical microscopy, and is a key factor in determining the end use of the material. Therefore, carbon product precursors are deemed graphitizable or non-graphitizable, based on the isotropy or anisotropy present in the material after carbonization (Figure 2.1). A graphitizable carbon will, under high-temperature treatment (up to $3300^{\circ} \mathrm{K}$ ), convert to graphite, which is described as soft carbon, anisotropic in properties, and possessing low surface areas and little porosity. Nongraphitizing carbons, however, do not develop any extensive crystalline graphite lattices upon heating to $3300^{\circ} \mathrm{K}$, and are described as hard carbons, isotropic in bulk properties, with appreciable surface area and pore volume within a micro-porous network ${ }^{(5)}$. 

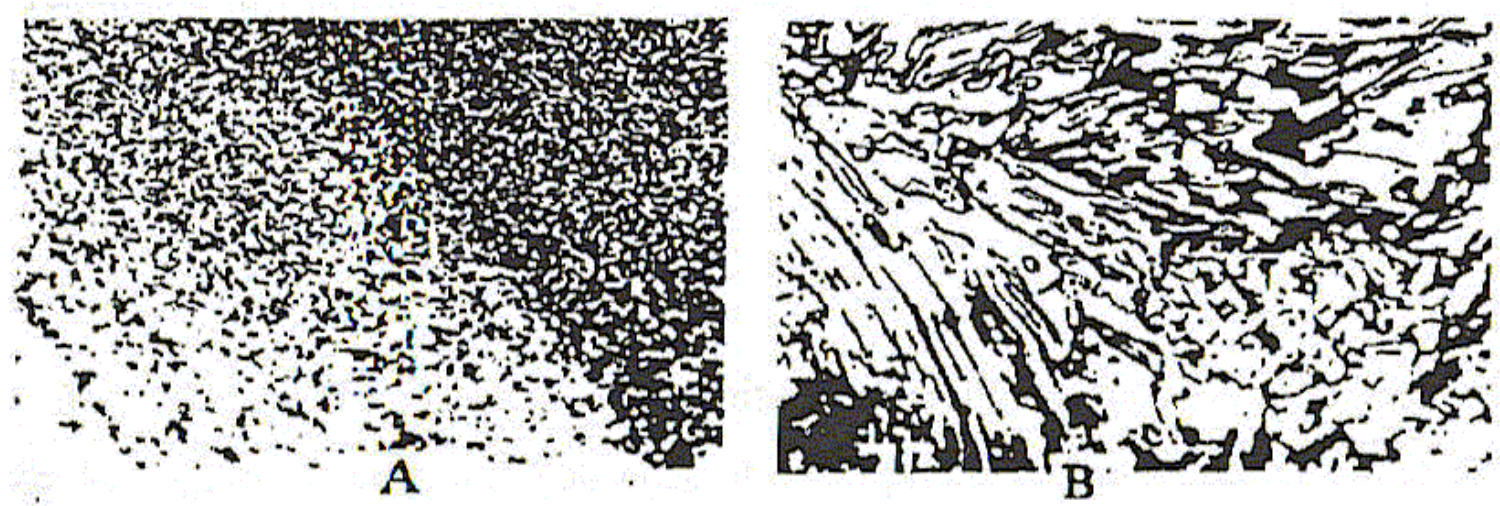

Figure 2.1 Optical Textures of A) Isotropic and B) Anisotropic Cokes ${ }^{(6)}$

One of the larger scale uses of carbon products is in the aluminum industry, which is a $\$ 33$ billion dollar industry in America alone. This industry revolves around the use of carbon anodes, which are used in the production of aluminum metal as it is derived from alumina. The electrochemical process uses the anode as a source of carbon, and during the cell operation the carbon anode is sacrificially converted to carbon dioxide according to the reaction:

$$
2 \mathrm{Al}_{2} \mathrm{O}_{3}+3 \mathrm{C} \rightarrow 4 \mathrm{Al}+3 \mathrm{CO}_{2}
$$

Since the production of aluminum metal is considerable, huge quantities of anode material are required each year. About $85 \mathrm{wt} \%$ of the anode is comprised of calcined petroleum coke and recycled anode butt material. These materials are typically held together with about 15 wt\% coal-tar binder pitch and baked into large solid blocks of carbon. Currently, approximately 2,000,000 tons of calcined petroleum coke and 746,000 tons of coal-tar pitch are utilized domestically in the rendering of aluminum. 


\subsection{Pitches}

Chief among materials that are used to produce carbon products of all kinds is the material known as pitch. Most people would hear the name "pitch" and think of a dark, thick, gooey mass of black, tar-like material. This is close, but for the purpose of this thesis it is important to have a clear understanding of what pitch actually is. Pitch is a manufactured organic material that is, along with materials known as tars, a member of a family known as bituminous materials ${ }^{(7)}$. Tars are usually the immediate precursors for pitch materials. Coal and petroleum are also members of this family, as are many other naturally occurring and manufactured materials. What distinguishes tars and pitches is, 1) they are man-made and 2) they are obtained through either thermal degradation or destructive distillation of organic precursors. These precursors can literally be almost any organic material, including wood, coal and petroleum. Each tar or pitch is distinct, due to the distinct nature of the precursor. Different woods will give different wood tars, and coals from two different mines will likely give different coal tars. The tars obtained are then further processed to remove some of the more volatile matter. After this is done, the heavy material remaining is referred to as pitch, which is a complex mixture of organic compounds, made up mostly of polycyclic aromatic hydrocarbons.

Coal-tar pitch is made during the production of metallurgical coke. Many years ago, coke production was done without the collection of the gases and vapors that are evolved during the heating of coal. This material would either condense into large pools of a black tar like substance, which could then leach into the soil around the oven, or drift off as a vapor and enter the ecological system. Since the development of recovery coke ovens, these volatiles are collected, along with any tar like materials. This coal tar is then 
distilled to remove some of the lighter compounds in the tar, which are used to make tar acids/bases, creosotes and naphthalenes. The heavy matter left after the distillation is called coal-tar pitch ${ }^{(8)}$ a residue with a required softening point, typically $110^{\circ} \mathrm{C}$.

Alternatively, pitch can also be made through the processing of petroleum, usually through catalytic cracking. The by-product heavy residue obtained from the cracking of petroleum can then undergo thermal treatment, vacuum or steam stripping, oxidation or distillation ${ }^{(9)}$. A specific type of pitch that can be produced, called mesophase pitch, is made when an isotropic pitch is subjected to careful thermal treatment. During this treatment, small pockets of mesophase, a liquid crystal state, form. As the treatment progresses, these pockets grow larger and coalesce. Eventually, the pitch becomes a $100 \%$ bulk mesophase pitch. These pitches have the properties of both an ordered solid state and a fluid liquid state, and are often used for high-modulus fibers and composites. However, consideration of mesophase pitches is beyond the scope of this study, and they will not be discussed any further.

Pitch use is determined by the structure and properties of the pitch, which are typically determined by the feed material (i.e. coal tar vs. petroleum) and the treatment used to convert this feed into pitch. Some examples of the characteristics of various commercial pitches are shown in Table 2.1. Some pitches are used as binders in the production of anodes used in the aluminum industry and electrodes used in the steelmaking industry. These binders help to strengthen the cokes and to hold the particles together, and should have high-carbon yields and low softening points. Other pitches can be used to reduce the porosity of carbon products, so these pitches should have low viscosities and ash contents. Pitches can also be the feed material in the production of 
carbon fibers. These pitches must have a low solids content, be highly reactive towards oxygen stabilization, and have a low enough softening point to allow for relatively easy spinning. Pitches can also be used as coke precursors, and the properties desired in the coke will determine the type of pitch used. This coke could be produced as metallurgical coke or as feed material for carbon anodes used in the aluminum industry.

\section{Table 2.1 Characteristics of Various Commercial Pitches ${ }^{(10)}$}

\begin{tabular}{|l|c|c|c|c|c|c|}
\hline & \multicolumn{3}{|c|}{ Binder Pitch } & \multicolumn{3}{c|}{ Impregnating Pitch } \\
\hline Supplier & Allied & Aristech & Koppers & Ashland & Kawasaki & Mitsubishi Kasai \\
\hline Feedstock Base & Coal Tar & Coal Tar & Coal Tar & Petroleum & Coal Tar & Coal \\
\hline Softening Point $\left(\mathbf{(}^{\mathbf{C}} \mathbf{C}\right)$ & 109.1 & 109.8 & 110.3 & 121.1 & 99.5 & 95.3 \\
\hline Coking Value (wt\%) & 58.5 & 57.6 & 58 & 49.1 & 50 & 44.3 \\
\hline Ash Content (wt\%) & 0.17 & 0.16 & 0.21 & 0.03 & 0 & 0.003 \\
\hline Sulfur (wt\%) & 0.61 & 0.62 & 0.59 & 3.1 & 0.41 & 0.43 \\
\hline Carbon (wt\%) & 93.84 & 92.84 & 93.83 & 91.25 & 92.7 & 92.49 \\
\hline Hydrogen (wt\%) & 3.66 & 4.42 & 3.92 & 5.08 & 4.44 & 4.27 \\
\hline \% H aromatic & 85.4 & 85.8 & 86 & 55.5 & 86.1 & 82.8 \\
\hline
\end{tabular}

All pitches must go through some additional treatment, as they are typically not usable directly after their immediate production. Typically, the main goal of the additional processing is to remove additional low molecular weight compounds, which affect the softening point, coke yield and viscosity. There are basically two different types of pitch treatment: physical treatment and chemical treatment.

Blending is the simplest of the physical methods of pitch pretreatment. Two pitches with different properties are blended in a ratio that results in the formation of a new pitch with property values that lie between the values of the two raw pitches. Another method of physically treating a pitch is through distillation. The more light materials that are removed from the pitch, the higher the softening point, density, and coke yield. 
A method of physically treating a pitch that has undergone much research, especially at West Virginia University, is solvent extraction. In this procedure, a solvent, typically N-methyl-2-pyrrolidone (NMP), is used to dissolve a pitch material, usually at the normal boiling point of NMP, $202^{\circ} \mathrm{C}$. This mixture is either filtered or centrifuged to remove any undissolved material, and then the NMP is removed from the soluble portion, usually by rotary evaporation. Mineral matter (ash) is removed during this process, along with high molecular weight materials which do not dissolve in the NMP. This could lower the softening point of the refined pitch product (due to the removal of the higher MW compounds). Additionally, and often more importantly, as the pitch is relatively ash free, this material is an ideal feedstock for the production of certain carbon products, namely pitches and cokes.

There are several different chemical methods of modifying pitches. Sulfur is used as a cross-linking agent in the chemical polymerization of pitches. Catalysts can be used in pitch treatment as well, and often compounds are added to pitches simply as an additive that adjusts the properties of the pitch. Thermal treatment of the pitch (different than distillation) is often used to treat pitches. Typically, the pitch is heated under an inert atmosphere, and this induces both the removal of lighter molecular weight compounds along with some cross-linking and other chemical changes.

In a recent study at West Virginia University by N. King ${ }^{(6)}$, thermal treatment of pitches using air blowing was studied. This method of pitch modification heats the pitch while introducing air into the mixture. A few volatile compounds are driven off from the pitch, while the air induces several chemical changes in the pitch. Cross-linking and dehydrogenation are among the different types of chemical effects that the pitch 
undergoes during the treatment. Air blowing is mainly used in the industry for producing asphalts, precursors for isotropic carbon materials, in addition to pitch precursors used for general purpose carbon fibers (GPCF) ${ }^{(11)}$. Little work has been undertaken on the application of air blowing for producing coker feeds from coal digests.

There has been extensive research into the effectiveness of air blowing in altering the properties of pitches. The softening point of a pitch is the most obvious property affected by this or other thermal treatments. The results of one study on air blowing a QI-free coal tar at three different temperatures proved that the softening point of a pitch does indeed increase with air blowing (Figure 2.2). It was also shown that at higher temperatures, the desired softening point of $280^{\circ} \mathrm{C}$ was achieved in shorter times ${ }^{(12)}$. Similar results using hydrogenated coal tars and petroleum tars were noted. It is believed that the pitches undergo an intramolecular linking during treatment. 


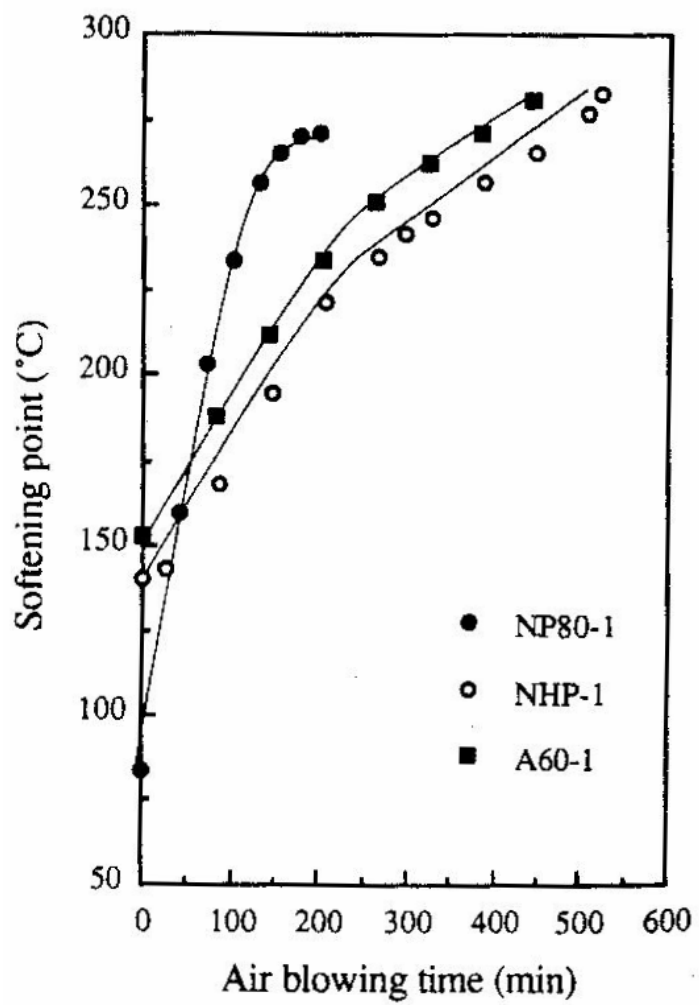

Figure 2.2: Softening point of coal tar (NP80-1), hydrogenated coal tar (NHP-1) and petroleum pitches $(\mathrm{A60-1})$ during air blowing at $330^{\circ} \mathrm{C} .{ }^{(13)}$

Coke yields are also affected significantly by air blowing. This is a key property, because a coker feed must retain most of the carbon during the coking process. In a study by Fernandez et al., it was found that coke yields increase dramatically with increasing time of air blowing ${ }^{(14)}$. This study also noted that the density and strength of cokes increased with increasing times of air blowing, and that, conversely, the porosity and reactivity of the cokes decreased with increasing air blowing time.

The viscosity and solubility of pitches are also greatly affected by air blowing. In a study on the viscosity of an impregnating coal tar pitch, air blowing caused an increase in the elastic behavior of a pitch at shear viscosities of $50 \mathrm{~Pa} / \mathrm{s}$. In contrast, the parent pitch showed a purely viscous behavior ${ }^{(15)}$. Choi et al. found that the toluene insoluble 
content of a coal tar pitch increased much more rapidly with air blowing than with nitrogen blowing ${ }^{(16)}$. Other studies verified that the solubility of pitches in general decrease with increased air blowing times.

For this thesis study, coal digests underwent air blowing at elevated temperatures. It was demonstrated in a previous study that this method of pitch treatment is very effective in modifying and controlling the properties of pitches. Additionally, this

method was studied in depth by N. King for pitch modifications ${ }^{(6)}$. However, it is unknown whether or not air blowing will be effective in coke feed production from coal digests.

\subsection{Coke}

Coke, made from the carbonization of coal, is a significant feedstock for the steel industry. Commercial coke production first started in the 1700's. Darby heated coal to drive off the volatiles and this process left a carbon rich solid called coke ${ }^{(17)}$. Iron was being extracted from iron ore previously by using charcoal, which is made from wood. Coke, it was discovered, was an even better source of carbon in this process. Iron ore is heated along with coke (carbon source) and the resultant oxidation/reduction reaction liberates the iron metal and releases carbon dioxide. Molten iron sinks in the blast furnace and the waste material (slag) is removed off of the top of the pool of material. Modern-day coke production occurs in large slot ovens that heat the coal under oxygen lean conditions. The coal is heated to temperatures as high as $2000^{\circ} \mathrm{F}\left(\sim 1093^{\circ} \mathrm{C}\right)$ for an extended time period (up to 18 hours). During this process, volatile organic materials escape from the coking chamber, and the solid material that is left is coke, which has a 
very high percent carbon. The amount of volatile material that comes off during the process depends of the type of coal that is used in the coking oven. Typically, the best type of coal for coke production is a medium-volatile bituminous coal, which usually contains approximately $20 \%$ volatile matter and as much as $85 \%$ carbon ${ }^{(17)}$. Coke comes out of the ovens and is usually water-quenched to cool it, and then it is shipped to the steel factories.

Old style coke "ovens" were simply mounds of coal with channels dug in them. Wood was stuffed into these channels and lit, and the heat generated from this fire would start the coking process. This would give a low yield of coke (20-30\%) and was very polluting. The volatile material that didn't combust in the process would condense and stream from the mounds. Later designs of coke ovens were called "beehive" ovens, and produced a much better yield of coke ( 65\%). However, there were no by-products, as most of the non-coke material would be combusted in the process as fuel. Most coke ovens in use today are designed similar to the Koppers By-Product Coke Oven. This oven can give a 75\% coke yield and takes only hours per batch, rather than days. The byproducts are pulled off of the ovens, collected, and processed into other useful products.

Coke is also made from petroleum heavy fractions. This heavy material is typically converted to coke using the delayed coking process. This is a thermal process designed to generate distillates at an elevated temperature (usually $470-487^{\circ} \mathrm{C}$ ) over a period of time (12 to $36 \mathrm{~h}$ ). Delayed coking is a combination of a continuous and batch process which produces gas, gasoline, gas oils, and coke. It is performed in a coking drum and is designed primarily to produce transportation fuels. The coker furnace itself has an inlet temperature between $343-357^{\circ} \mathrm{C}$ and an outlet temperature of around $500^{\circ} \mathrm{C}$. 
Most of the coke produced from petroleum is only usable as cement kiln fuel. Coke yields of this process vary from $20-40 \%$, depending on the feed types. About $20 \%$ of the total delayed coke produced is high-grade coke suitable for use by the aluminum industry in the production of the anodes that are utilized in the reduction of aluminum oxide to elemental aluminum. Even less is usable in graphite electrodes and some carbon-carbon composites ${ }^{(18)}$.

There are basically four types of petroleum coke: fuel, sponge, needle and shot. Fuel coke is used typically as a fuel for cement kilns or the utility industry. Worldwide, about $80 \%$ of the coke produced is fuel coke. Most of the remaining coke is sponge coke, which is calcined and sold to the aluminum industry. Needle coke is made from highly aromatic feed stock such as FCC decant oil or solids-free coal-tar pitches. This type of coke is used primarily in the steel industry. The formation of shot coke, used as packing materials and fillers, is not fully understood. It is usually the result of using heavier crudes and low recycle ratios in the processing, and is usually formed as small, hard pellets. This coke can also be used as a catalyst in the titanium dioxide industry ${ }^{(18)}$.

The end use of a coke is directly influenced by the properties of the material that is used to make the coke. Like pitches, there are both anisotropic cokes, which have large crystalline domains, and isotropic cokes, which are devoid of any long-range order in the structure (refer to Figure 2.1). Anisotropic cokes are used to manufacture graphite electrodes and carbon anodes. These artifacts can carry huge electrical currents at very high temperatures. Isotropic cokes are more suited for nuclear graphites, which require high chemical purity, necessary to prevent the adsorption of low energy neutrons. Also, 
the bulk isotropic nature of the nuclear-grade graphite gives it a high degree of dimensional stability when heated, due to the lack of any preferred dimensional changes.

\subsection{Carbon Products Source Materials}

Due to the fact that most, if not all, carbon products are made today from either coal or petroleum, a more detailed look at each of them would be beneficial. As this study is concerning the production of coke precursors from coal, knowledge of coal would be useful in the analysis of the results. Also, petroleum, coal's chief competitor in the carbon products industry, is examined and the two are compared.

\subsubsection{Coal}

While the first use of coal is not exactly known, it is believed that the first large scale use of coal was in China over thousands of years ago ${ }^{(17)}$. Marco Polo helped spread the knowledge of coal uses after his stay in China in 1295. Coal mining has actually led to some very significant inventions over the years, such as the steam engine, used to pump water out of deep mines to prevent flooding, and coke as an iron-smelting carbon source, which led to the production of more affordable iron.

Coal is formed from plants that have undergone millions of years of chemical and geological alteration ${ }^{(17)}$. The process that turns the remains of plants into coal is called coalification. Plants die and fall to the ground, where, under certain conditions, much of this matter is covered with water. These peat beds are the beginning of the biochemical phase of coalification. During this time, hydrogen eating microbes begin to digest some of this matter. After a time, the peat is completely covered with inorganic material, and 
the organics are compressed. The pressure and temperature both increase as the depth of this material increases. Next, the geochemical phase begins, where the hydrogen and oxygen concentrations are decreased, while the carbon concentration increases. As the coal forms, the material undergoes a variety of chemical reactions; these include dealkylation, aromatization, and condensation. After a very long time, the organic matter could eventually become graphite. The progression of coal matter generally follows the following diagram,

\section{Peat $\rightarrow$ Lignite $\rightarrow$ Sub-Bituminous Coal $\rightarrow$ Bituminous Coal $\rightarrow$ Anthracite}

Each successive level on the above diagram is a different rank of coal. Peat and lignitic coal both have undergone significantly less coalification than the other higher ranks, and the carbon content of these is about $60 \%$ and $70 \%$, respectively ${ }^{(17)}$. Bituminous coals usually have around $85 \%$ carbon, and they have lost a great deal of the oxygen in the molecular structures of the original material, down to a value of about $7 \%$. Sub-bituminous coal has properties between bituminous and lignite. Anthracite, the highest rank coal, is the most coalified. This coal typically has a carbon concentration as high as 95\%. The ASTM standards for ranking coals are given in Table 2.2. 
Table 2.2 ASTM Std. D 388-66 System for Coal Rank Classification ${ }^{(17)}$

\begin{tabular}{|c|c|c|c|c|}
\hline Class & Group & Fixed Carbon ${ }^{a}$ & Volatile Matter $^{a}$ & Heating Value $^{b}$ \\
\hline \multirow[t]{3}{*}{ Anthracite } & Meta-anthracite & $>98$ & $<2$ & \\
\hline & Anthracite & $92-98$ & $2-8$ & \\
\hline & Semi-Anthracite & $86-92$ & $8-14$ & \\
\hline \multirow[t]{5}{*}{ Bituminous } & Low Volatile & $78-86$ & $14-22$ & \\
\hline & Medium Volatile & $69-78$ & $22-31$ & \\
\hline & High Volatile A & $<69$ & $>31$ & $>14,000$ \\
\hline & High Volatile B & & & $13,000-14,000$ \\
\hline & High Volatile C & & & $11,500-13,000$ \\
\hline \multirow[t]{3}{*}{ Sub-Bituminous } & Sub-Bituminous A & & & $10,500-11,500$ \\
\hline & Sub-Bituminous B & & & $9,500-10,500$ \\
\hline & Sub-Bituminous C & & & $8,300-9,500$ \\
\hline \multirow[t]{2}{*}{ Lignite } & Lignite A & & & $6,300-8,300$ \\
\hline & Lignite B & & & $<6,300$ \\
\hline \multicolumn{5}{|c|}{ a - The fixed carbon and volatile matter percentages are determined on a dry, mineral-free basis. The mineral matter is calculated from the ash conten } \\
\hline \multicolumn{5}{|c|}{ by the Parr formula: mineral matter $=1.08[$ percent ash +.55 (percent sulfur)]. $\mathrm{b}-$ The heating value, reported in BTU/b, is expressed on a moist, } \\
\hline \multicolumn{5}{|c|}{ mineral-free basis. The moisture content is the bed moisture or equilibrium moisture of the coal after equilibration with nominally $100 \%$ relative } \\
\hline \multicolumn{5}{|c|}{ humidity atmosphere. Some overlap occurs in the heating-value range of sub-bituminous and high volatile $\mathrm{C}$ coals. Coals with heating values between } \\
\hline
\end{tabular}

Since coal is derived from plant matter, and since a great many plants are significantly different from each other and the conditions of deposition vary, coals of the same rank can often differ greatly. Microscopically, coals can be differentiated by examining the banded components, classified by Stopes in 1919, called lithotypes ${ }^{(19)}$. The lithotypes vitrain, clarain, durain and fusain, can be further broken down into smaller microscopic groups called macerals. The three main types of macerals are vitrinite, exinite and inertinite. Vitrinite macerals are typically derived from more woody tissues, while exinites come from the resins, fatty secretions, cuticles and spores from the plants. Inertinites are derived from the plant matter that has been partially carbonized during the peat stage of coalification. These macerals, studied by scientists called petrographers, are important in the study of the processing of coal, such as in the determination of soluble or convertible portions of the coal. 
In addition to carbon, coals contain hydrogen, oxygen, sulfur and nitrogen, as well as inorganic mineral matter, depending on the original plant material and depositional environment. Though there is no real molecular formula for coal, there are estimations (Figure 2.3). Higher rank coals typically have a higher percentage of carbon, and lower rank coals have more heteroatoms (Table 2.3).

To determine the composition of the coal, both elemental and proximate analyses are used. Elemental analysis, described in greater detail in a later section, gives the percentages of carbon, hydrogen, oxygen, nitrogen and sulfur. Proximate analysis will give the percent moisture, percent volatile matter, percent fixed carbon and the percent ash in a sample. The inorganic matter in the original coal will be converted to ash (metal oxides) during any combustion process.

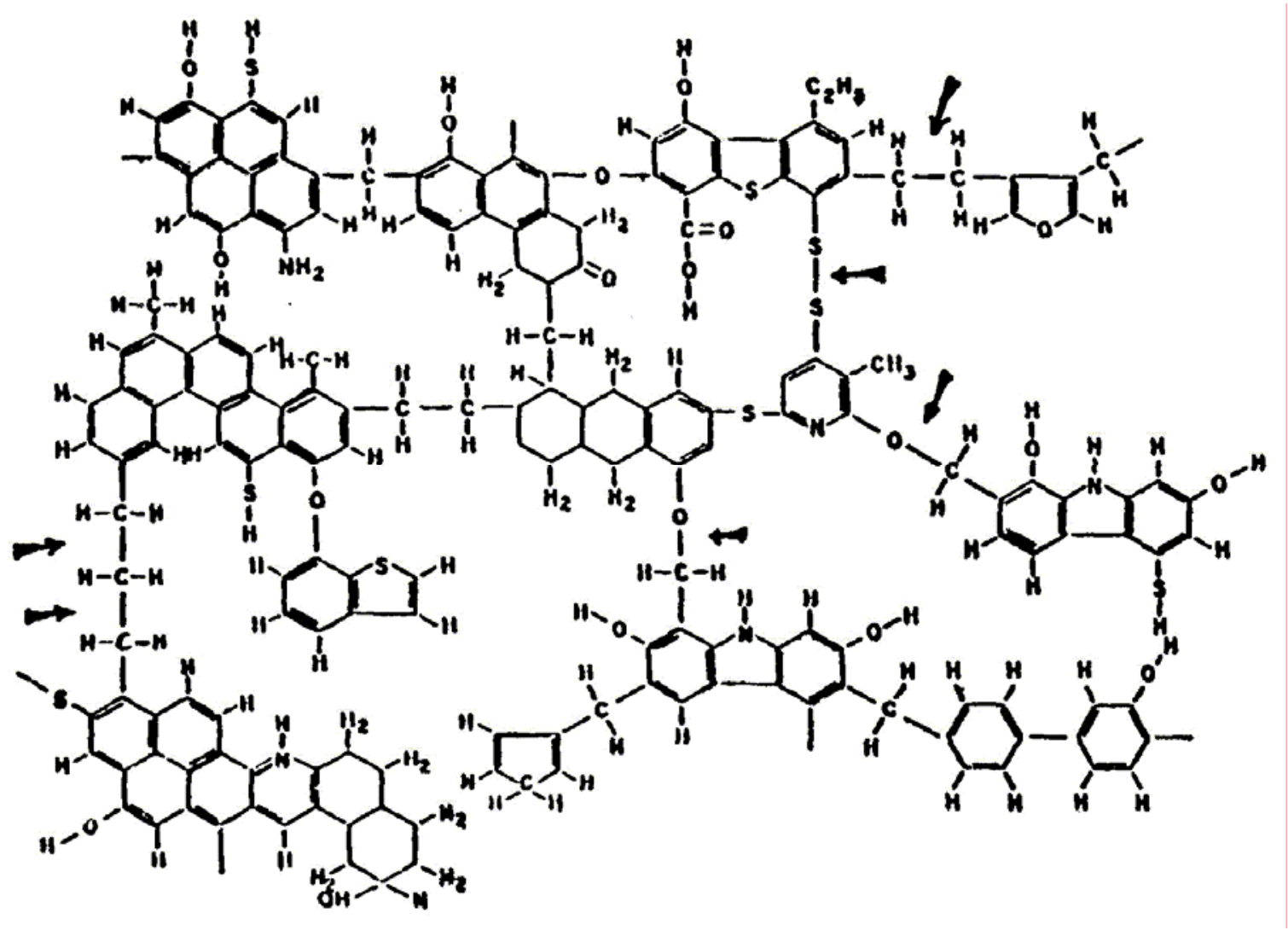

Figure 2.3 One Theoretical Molecular Unit of Coal ${ }^{(20)}$ 
Table 2.3 Percent Elemental Composition of Various Coal Ranks ${ }^{(21)}$

\begin{tabular}{l|ccccc|}
\cline { 2 - 6 } Sample & \multicolumn{5}{c|}{ Element, \% wt (dry, ash-free basis) } \\
\cline { 2 - 6 } & C & H & O & N & S \\
\hline Meta-Anthracite & 97.90 & 0.21 & 1.70 & 0.20 & 0.00 \\
Anthracite & 95.90 & 0.89 & 1.80 & 0.30 & 1.80 \\
Semi-ahthracite & 90.50 & 3.90 & 3.40 & 1.50 & 0.70 \\
Low Volatile Bituminous & 90.80 & 4.60 & 3.30 & 0.70 & 0.60 \\
Medium Volatile Bituminous & 89.10 & 5.00 & 3.60 & 1.70 & 0.60 \\
High Volatile Bituminous A & 84.90 & 5.60 & 6.90 & 1.60 & 1.00 \\
High Volatile Bituminous B & 81.90 & 5.10 & 10.50 & 1.90 & 0.60 \\
High Volatile Bituminous C & 77.30 & 4.90 & 14.30 & 1.20 & 2.30 \\
Sub-Bituminous A & 78.50 & 5.30 & 13.90 & 1.50 & 0.80 \\
Sub-Bituminous B & 72.30 & 4.70 & 21.00 & 1.70 & 0.30 \\
Sub-Bituminous C & 70.60 & 4.80 & 23.30 & 0.70 & 0.60 \\
Lignite & 70.60 & 4.70 & 23.40 & 0.70 & 0.60
\end{tabular}

\subsubsection{Petroleum versus Coal}

Webster has defined petroleum as “a dark brown or greenish inflammable liquid, which, at certain points, exists in the upper strata of the earth, from whence it is pumped or forced by pressure of the gas attending it. It consists of a complex mixture of various hydrocarbons, largely of the methane series, but may vary much in appearance, composition, and properties. It is refined by distillation, and the products include kerosene, benzene, gasoline, paraffin, etc.” ${ }^{(22)}$. For this discussion, the important issues regarding petroleum are its differences from coal. Chief among these differences is the molecular weight and the carbon-to-hydrogen ratio. Petroleum samples typically have a molecular weight range of 150 to $250^{(23)}$ and $\mathrm{C} / \mathrm{H}$ ratios between 0.53 and $0.73^{(24)}$. Coal however, typically has molecular weights in excess of 1000 , while the $\mathrm{C} / \mathrm{H}$ ratio is higher than petroleum, with an average value of 1.25.

Petroleum has become the feed stock of choice for most carbon products, due to the lower carbon-to-hydrogen ratio. This causes the petroleum to be less viscous than 
coal liquids, thus transportation is easier, and processing of the liquid petroleum is easier than processing coal, a solid. However, the quality of the petroleum feedstocks is becoming more and more suspect, and the supplies of petroleum in the world and especially in the US are being depleted at an alarming rate. The concentrations of sulfur, nickel and vanadium in existing supplies of petroleum are leading to advanced deterioration in carbon products, specifically anodes and electrodes. These metals and sulfur cause the lifetime of the product to decrease greatly. Additionally, petroleum prices have risen over the past several years to record highs, and this trend is not expected to end anytime soon, due to the continued depletion of the world's oil supplies and the political and cultural instability of the Middle East. Coal is relatively cheap, and is available domestically to a much greater extent than petroleum. Using coal as a source for carbon products would lessen the country's dependence on foreign oil sources for things other than fuels and petrochemicals.

\subsection{Processing Coal for Use in Producing Carbon Products}

\subsubsection{Pyrolysis}

There are typically only two methods to process coal for use in carbon product manufacturing: either pyrolysis or liquefaction. Pyrolysis is a process where coal is heated up to high temperatures in the absence of oxygen, to prevent combustion. This process drives off volatile compounds, which are collected and condensed into a hydrogen-rich liquid, leaving a carbon rich solid residue (either char or coke). The heating causes bonds to break in the coal, initially causing the aromatic cluster units to break apart at temperatures around $400^{\circ} \mathrm{C}$. As the temperature rises over $450^{\circ} \mathrm{C}$, the 
aliphatic side chains and lower molecular weight coal fragments begin to break apart and volatilize. These are the compounds that become the tars and gases that are evolved during the pyrolysis, and subsequently this coal tar is collected for further treatment. As the process nears completion, the residue remaining is formed from the aromatic clusters re-polymerizing at the elevated temperatures. Pyrolysis results in the formation of char or coke, which contains the mineral matter present originally in the coal. The coal tar, which is richer in hydrogen than the original coal, is typically processed further after it is collected, such as desulfurization. This coal tar is then used in a wide variety of products.

\subsubsection{Indirect Liquefaction}

Liquefaction, as the name implies, is the process of liquefying coal, either through the addition of a solvent (direct) or through first completely breaking down the coal into synthesis gases (mainly $\mathrm{H}_{2}$ and $\mathrm{CO}$ ) and then recombining them into liquid fuels (indirect). Indirect liquefaction is also referred to as gasification. The process of recombining the gases usually occurs in the presence of a Fischer-Tropsch catalyst so the various higher hydrocarbons are formed. It has the advantage of minimizing the amount of mineral matter present in the final product. The properties of different FischerTropsch catalysts allows for the final products to be highly preferential towards gas, gasoline, kerosene, diesel fuel, fuel oil, methanol or acetone. The chief disadvantage of the process is the production of a variety of by-products. This is due to the fact that the gasification of the coal is typically done with steam and oxygen. Additionally, this is an expensive process in terms of the thermal efficiency in the destruction of the original coal structure. 


\subsubsection{Direct Liquefaction}

Direct liquefaction can occur through either dissolution of the coal in an organic, hydrogen-donor solvent or through the catalytic hydrogenation of the coal. Catalytic hydrogenation of coal is not covered in this present research; thus, the discussion hereafter will pertain only to the dissolution method. Direct liquefaction is the process of dissolving the coal in an organic solvent, which is typically also a hydrogen-donor solvent. The mixture is heated and stirred to a temperature high enough to promote the breakdown of the coal molecules and the transfer of hydrogen from the solvent (or gaseous atmosphere) to the coal fragments. Hydrogen caps the coal radical fragments which are created from the heating and dissolution. Direct liquefaction results in a liquid product and, typically, a solid residue. This residue is usually concentrated with the mineral matter from the original coal, along with "unconverted" coal. This unconverted portion of the coal is made up of larger molecular weight species that do not break down during the process.

\subsubsection{Direct Liquefaction Mechanisms}

The specific mechanism that takes place during direct liquefaction is the subject of some discussion. Most researchers agree that it occurs via some type of free radical mechanism, but the exact form of this mechanism is disputed. According to research by

Curran et al. ${ }^{(25)}$ and Vernon ${ }^{(26)}$, hydrogen is transferred to the coal from the solvent in this free radical process. The formation of the free radicals occurs during the heating of the coal-solvent mixture. The radicals that are formed as the coal structure thermally degrades are capped in the most thermodynamically favorable process. The propositions 
and observations made by Curran et al. have been simplified down into a set of five basic reactions, which form the framework of much of the discussion of the mechanism of liquefaction (Figure 2.4).

$$
\begin{aligned}
\text { Coal } & \rightarrow 2 \mathrm{R} \cdot \\
\mathrm{R} \cdot+\mathrm{DH}_{2} & \rightarrow \mathrm{RH}+\mathrm{DH} \cdot \\
\mathrm{R} \cdot+\mathrm{Coal}-\mathrm{H} & \rightarrow \mathrm{RH}+\mathrm{Coal} \cdot \\
\mathrm{R} \cdot+\mathrm{DH} \cdot & \rightarrow \mathrm{RH}+\mathrm{D} \\
\mathrm{R} \cdot+\text { Coal-H } \cdot & \rightarrow \mathrm{RH}+\text { Coal }
\end{aligned}
$$

\section{Figure 2.4 Proposed Free Radical Mechanism of Liquefaction ${ }^{(25)}$}

It is believed that the rate-controlling step here is the thermal dissociation of the labile bonds in the coal, which generates two free radical species $(\mathrm{R} \cdot)$. These radicals may then abstract hydrogen from the donor solvent $\left(\mathrm{DH}_{2}\right)$, from the coal or from the free radicals that are produced by either of these two steps ${ }^{(25)}$.

According to research conducted by Wiser ${ }^{(27)}$ the radicals formed during direct liquefaction are capped in one of three possible ways. The first method is the addition of hydrogen atoms to close the radical end of the coal fragment. This method is obviously the desired result in this process, as the liquid product needs to have a lower $\mathrm{C} / \mathrm{H}$ ratio than the feed coal. The larger coal molecules are broken up during the process and then capped with hydrogen to form smaller, more soluble and more hydrogen-rich species. Another possible method that radicals are capped is through the intra-molecular rearrangement of atoms within the free radical. The third method occurs when the free radical undergoes polymerization with other radicals formed during the breakdown of the

coal structure. Both the second and third method of capping the free radicals results in formation of undesirable species. The formation of char or coke-like materials occurs 
mostly through the third method. In controlling these reactions, it is essential to design the process in such a way that there is an excess of hydrogen available to cap the radicals that have been formed.

A slightly different mechanism has been proposed for coal liquefaction by Malhotra and McMillen ${ }^{(28,29)}$. They state that while there is a free radical mechanism, it does not account for all of the coal conversion that occurs in the process. According to their theory, if a solvent is used to quench the radicals, its ability as a liquefaction solvent should correlate with the weakness of its carbon-hydrogen bonds. The researchers found, however, that solvents of equal ability as radical scavengers (such as indane, ethylbenzene and tetralin) were not necessarily equally effective in their ability as a liquefaction solvent ${ }^{(29)}$. They proposed that solvents not only play a role by stabilizing radicals (which is the traditional theory), but they are also active participants in cleaving molecular bonds. The solvent donates a hydrogen atom into a particular position on a large coal molecule, and this hydrogen atom causes the strong bond to be cleaved and this creates additional radicals (Figure 2.5).

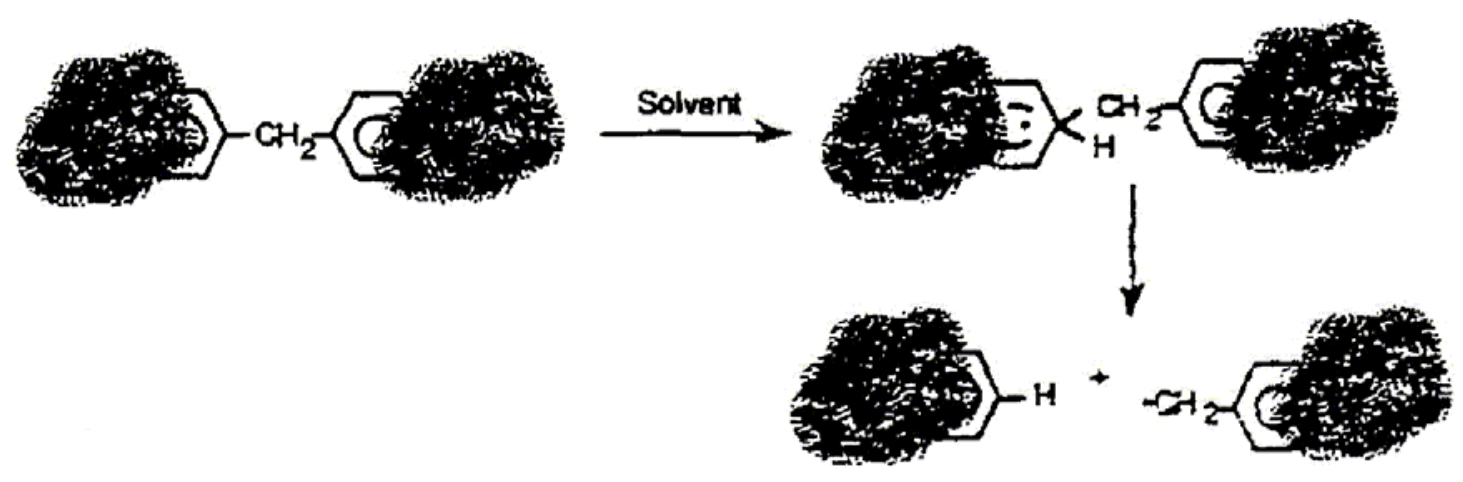

Figure 2.5 Solvent Mediated Hydrogenolysis of a Strong Linkage in Coal ${ }^{(29)}$ 
The hydrogenation process, according to Berkowitz ${ }^{(30)}$, occurs through a complicated system of reactions, reversible reactions and side reactions, as can be seen in Figure 2.6. The process is broken down into two parts, solubilization and then hydrogenation. The soluble portions of the coal are dissolved in the solvent, to varying degrees depending on the content and makeup of the coal. After this, secondary hydrogenation occurs, based on the specific reaction conditions. As the process continues, certain coal fragments are converted to lower and lower molecular weight species (i.e. oils), but there are also polymerizations and condensations which take the lower molecular weight species and turn them into substances more like char and coke. If the process is properly controlled, a large portion of the coal is recovered in the oil fragment, depending on the solvent used. Often these oils are used as fuel sources. However, this thesis research is concerned with making coke precursors, so the goal is not necessarily to make all oils, but to simply convert as much of the coal as possible into lower molecular weight species (i.e. asphaltenes and oils). The different liquids formed in coal liquefaction are defined in Table $2.4^{(30)}$. 

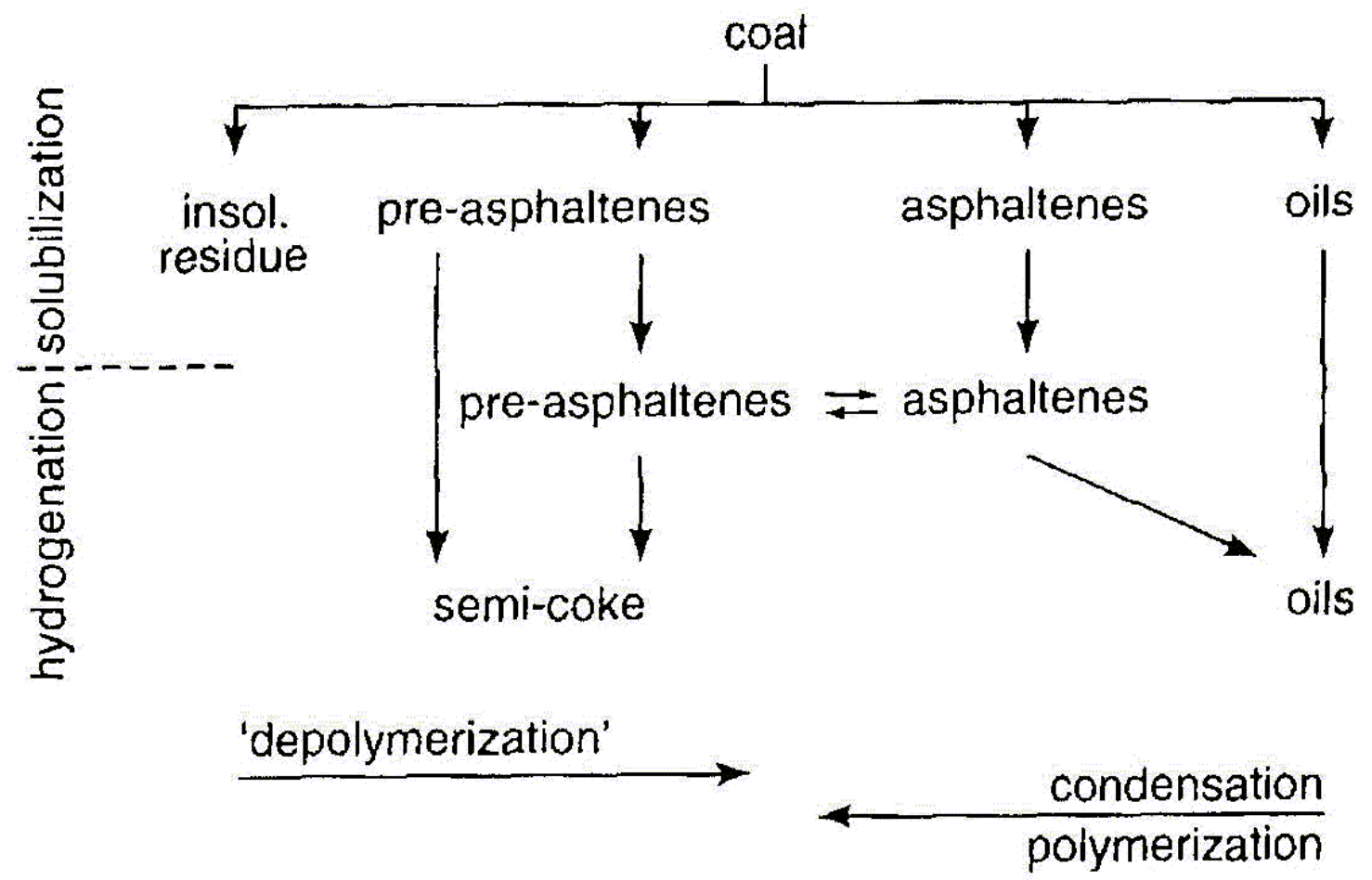

Figure 2.6 Conceptual Reaction Sequences in Coal Liquefaction ${ }^{(30)}$

Table 2.4 Definition of Primary Liquids from Berkowitz ${ }^{(30)}$

\begin{tabular}{|c|c|c|}
\hline Liquid & Soluble In & Insoluble In \\
\hline Carbenes & Carbon Disulfide & n-Pentane \\
\hline Carboids & n-Hexane & Carbon \\
\hline Asphaltenes & Benzene or Toluene & n-Hexane \\
\hline Preasphaltenes & Tetrahydrofuran & Benzene \\
\hline
\end{tabular}

Much of the theory on coal liquefaction points towards a stepwise mechanism in which hydrogen is transferred one atom at a time. However, the possibility exists that simultaneous, concerted transfers of two hydrogen atoms occurs and is in fact a significant portion of what takes place in direct liquefaction. In work by Virk et al., it is proposed that these pericyclic reactions play a larger role in liquefaction than the homolytic scissions ${ }^{(31)}$. However, pathways for model reactions investigated under 
liquefaction conditions thus far do not seem to bear up under the weight of experimental results. This does not mean, though, that these reactions cannot play a role in coal liquefaction, just that the means to reveal their possible consequences have not yet been devised.

Much information on liquefaction parameters has been obtained over the years from reactions with coal and a model hydrogen donor solvent, often tetrahydronaphthalene, or tetralin. Coal and tetralin are reacted together to form coalderived liquid products and a significant portion of naphthalene. The naphthalene forms from the tetralin surrendering four of its hydrogen atoms to the coal molecules during the reaction. The tetralin could also simply give up its hydrogen atoms to form hydrogen

gases, which are often developed during the liquefaction process ${ }^{(32)}$. Naphthalene could also be formed from the combination of naphthalene and other molecules found in the coal by combination to release methane (from methylnaphthalene, for instance) ${ }^{(30)}$.

\subsubsection{Direct Liquefaction Parameters}

The properties of coal that affect the results of liquefaction have been reported, through many laboratory studies and pilot plant operations. The chief factors affecting the liquefaction process include the coal rank, ash content, moisture content, oxygen content, particle size and hydrogen content. These properties are summarized in the following table (Table 2.5). 
Table 2.5 Fundamental Properties Important in Coal Liquefaction ${ }^{(30)}$

\begin{tabular}{|c|c|c|}
\hline Property & Influence & Desired Level \\
\hline \hline Rank & Liquids Yield & Medium \\
\hline Ash Content & Operations and Handling & Low \\
\hline Moisture content & Thermal efficiency & Low \\
\hline Hydrogen content & Liquids yield and hydrogen & High \\
\hline Oxygen content & Gas make and hydrogen consumption & Low \\
\hline Extractability & Liquids yield and quality & High \\
\hline Aliphatic character & Liquids yield and quality & High \\
\hline Reactive Macerals & Liquids yield & High \\
\hline Particle Size & Operations & Fine to very fine \\
\hline
\end{tabular}

The parameters that affect the ability of a coal to be processed by direct liquefaction have also been determined over many studies and tests. The composition of the coal, type of solvent, mineral matter in the coal and the presence of hydrogen gas all affect the success or lack thereof during direct liquefaction.

The composition of the coal and how it affects liquefaction has been studied extensively and there have been a variety of results. Most of the differences arise from the very heterogeneous nature of coal One rank of coal from one geographic region can have a widely different composition than the same rank of coal from a different geographic region. The petrographic composition of the coal can also vary from coal to coal. This is based on the vegetation components that are incorporated in the coalification process. The amount of biochemical degradation of organic matter before the coalification process occurs can also alter the coal composition.

One of the more basic results of studies that have been conducted is that coals with more than $89 \%$ carbon content were unsuitable for hydrogenation and gave a low liquid yield because of the larger, more condensed polynuclear structures ${ }^{(33)}$. High 
volatile bituminous coals, however, were found to be the best coals for liquefaction, while low rank coals such as lignite and sub-bituminous coals gave lower liquid yields and were affected more by the reaction conditions than the other coals.

It has also been found that the coal rank can affect the mechanism of coal liquefaction ${ }^{(34)}$. Keogh and Davis found in their research that both bituminous and subbituminous coals have two distinct liquefactions stages. For bituminous coals, as conversion increases in the initial stage, the production of asphaltenes and preasphaltenes increase while oil and gas production remains constant. In the second stage, a maximum conversion is achieved, and at this point the asphaltenes and preasphaltenes begin to be converted to oils and gases. For sub-bituminous coals, the production of oil and gas increases as well as the asphaltenes and preasphaltenes in the first stage, while the second stage is essentially the same as for bituminous coals (Figure 2.7). 


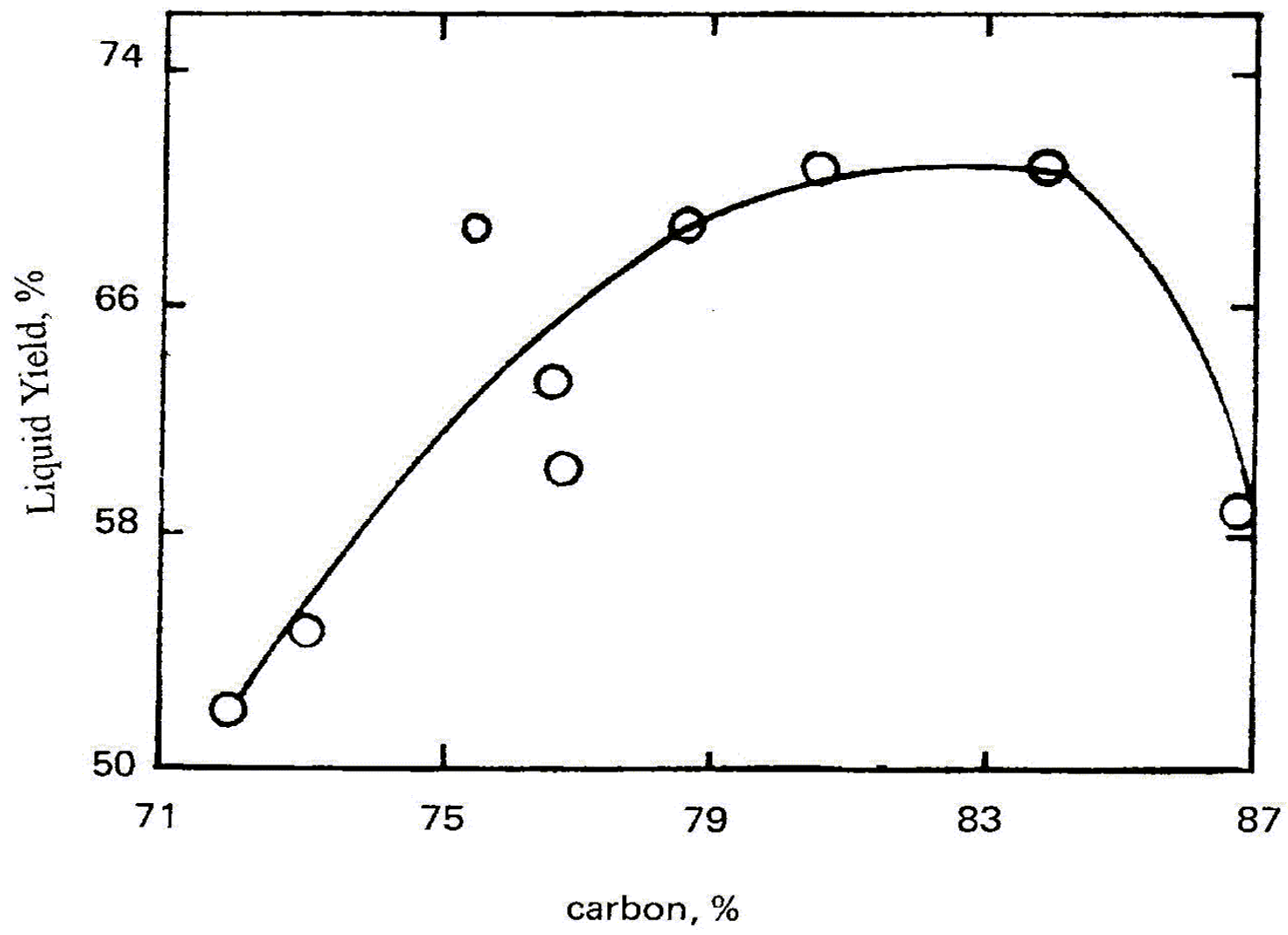

Figure 2.7 Effect of Carbon Content on Liquid Product Yield ${ }^{(33)}$

The petrographic composition of a coal can also affect the conversion from liquefaction. Each maceral type has a different behavior during liquefaction. Keogh et al. ${ }^{(35)}$ found that, after separation of the maceral groups through gradient centrifugation, at temperatures below $385^{\circ} \mathrm{C}$ the conversions are essentially the same. As the temperature of the process increases, the liptinites were found to be converted more than the vitrinites, which were in turn converted more than the inertinites. However, they also noted that the weighted sum of the conversions of the macerals in a coal do not add up to the actual conversion of the parent coal. This implies that there is some synergistic effect when the maceral groups are in the presence of each other. This research is somewhat substantiated through research from Cloke and Wang ${ }^{(36)}$, who noted that the vitrinites 
were hydrogenated by $350^{\circ} \mathrm{C}$, and that most liptinites were converted by $400^{\circ} \mathrm{C}$. They also found that inertinite could be partially converted with temperatures over $400^{\circ} \mathrm{C}$, but that this was not necessarily beneficial, as the rate of re-polymerization becomes more significant than the rate of hydrogenation. Given et al. ${ }^{(37)}$ found that as the reactive maceral content of a sub-bituminous coal increased, the yield from a solvation process increased (Figure 2.8).

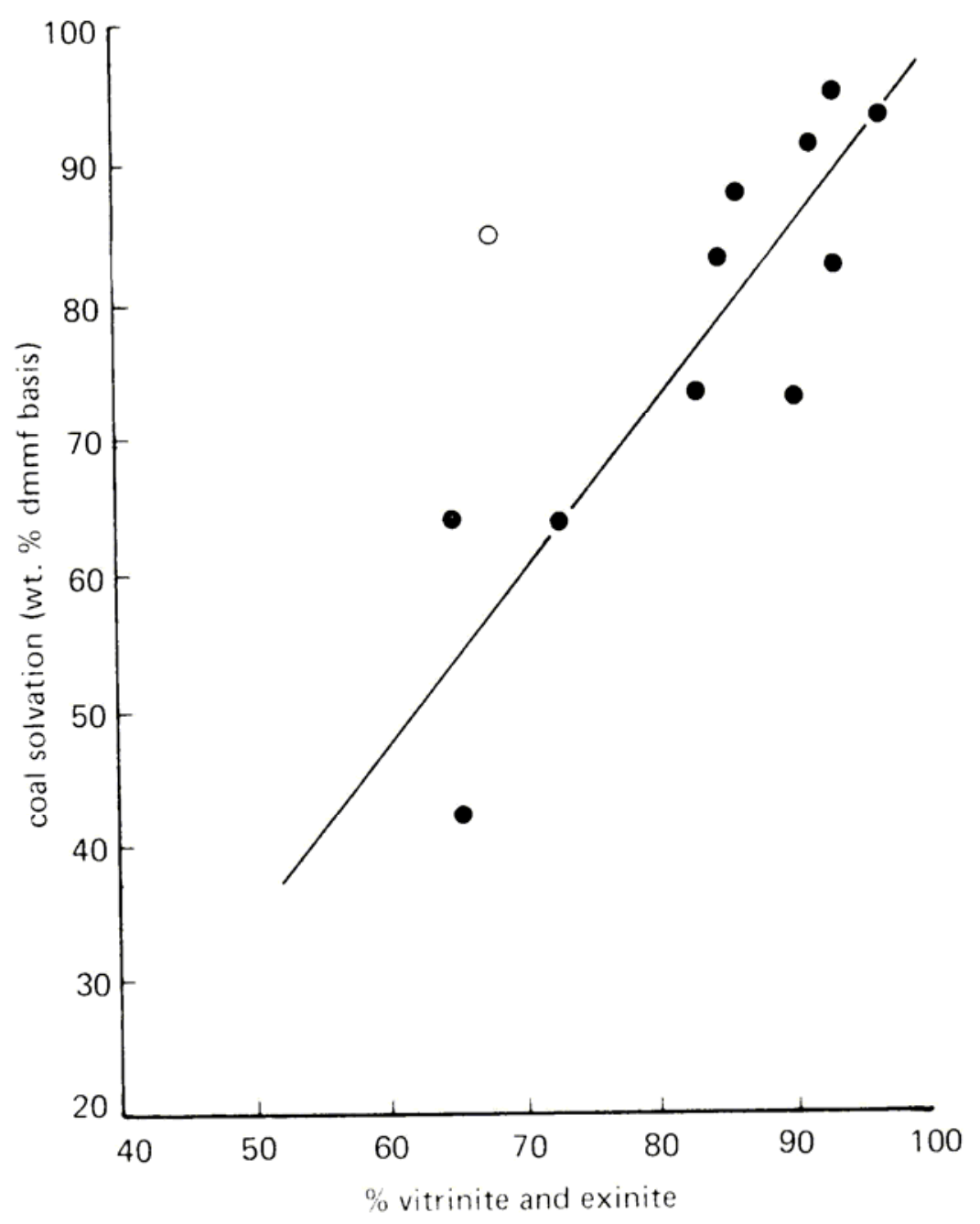

Figure 2.8 Dependence of solvation yield from a sub-bituminous coal on the reactive maceral content ${ }^{(37)}$ 
The selection of the solvent to use in the liquefaction process can obviously have an enormous effect on the process success. This can affect not only the conversion, but also the amount of consumed hydrogen, the quality of the liquid products and the degree and quantity of the retrograde reactions that will occur ${ }^{(38)}$. The selection of liquefaction solvent is done so that the most efficient transfer of hydrogen to the free radicals formed during the thermal decomposition can be reached. According to Whitehurst et al ${ }^{(39)}$, there are four chemical properties that can influence the ability of a solvent to be an effective liquefaction solvent. They are: 1) the hydrogen donor capacity of the solvent, 2) the physical solubilization of the coal products in the solvent, 3) hydrogen transfer capacity, or the ability of the solvent to "shuttle" hydrogen from a gaseous atmosphere to the coal fragments, and 4) the presence of species that promote the formation of char and coke.

The ability of a solvent to donate hydrogen is thought of as the key parameter in its ability to convert coal successfully in direct liquefaction. The labile hydrogen present in the solvent helps in stabilizing the free radicals that are formed during the initial steps of the liquefaction. A solvent with a greater ability to give up hydrogen will traditionally be the better liquefaction solvent. However, this is not always the case, as the other solvent properties are important in liquefaction.

The ability of hydrogen donors or shuttling agents to solvate and / or swell the coal structure is widely recognized as being beneficial to liquefaction. In a study made by Shibaoka ${ }^{(40)}$, vitrinite macerals were heated under hydrogen pressure at moderate temperatures $\left(305-390^{\circ} \mathrm{C}\right)$ for $1 \mathrm{hr}$ each in three solvents, tetralin, naphthalene and decalin. It was observed that the tetralin causes the vitrinite particles to swell, and certain 
portions became plastic and formed spheres termed vitroplasts. In the presence of naphthalene, the vitrinite macerals were also greatly expanded but did not become plastic. Finally, in decalin, vitrinite was neither greatly expanded nor did it become plastic. These solvents are known to have decreasing success as liquefaction solvents in the order of tetralin, naphthalene and decalin. While hydrogenation and dissolution may occur on the surfaces of the vitroplasts, polymerization may occur simultaneously in the hydrogen starved interior, and thus dissolution capacities may improve the contact between donor solvent and the reacting mass of coal to the benefit of liquefaction yields. Miller et al. suggest that the optimum hydrogen content of coal-derived solvents decreases as their aromaticity increases. Less hydrogen would be required to bring the more aromatic of these parent coal-derived solvents to their optimum donor potential ${ }^{(41)}$.

Hydrogen shuttling can be thought of as any reaction in which an organic compound acts as an agent for the transfer of hydrogen to coal either from the gas phase or from other organic compounds that may undergo dehydrogenation. Typically, the transfer compounds are thought to be relatively large polynuclear aromatics. Condensed aromatics are recognized as assisting in liquefaction by physical solvation. Davies et al. noted that the standard extraction efficiency measured for non-donor aromatic hydrocarbons in small tubing bombs generally increases with the number of condensed aromatic rings present in the solvent ${ }^{(42)}$. Reactions run at West Virginia University and by others support the idea that liquefaction conversion is increased with hydrogen pressure, indicating that the solvent acts as a shuttling medium for hydrogen in the gas phase to get to the coal. 
Solvents have been arranged into four groups ${ }^{(43)}$ based on their effects on coals. There are non-specific, specific, degrading and reactive solvents. Of the four solvent types, the specific and reactive solvents are of significance to the topic of direct coal liquefaction. Specific solvents will typically dissolve 20 to $40 \%$ of the original coal at temperatures about or below $200^{\circ} \mathrm{C}$. These solvents are electron donors, and the use of these solvents is simply a physical solution process. The solvents most commonly associated with this type are pyridine and N-methyl-2-pyrrolidone (NMP). Degrading and reactive solvents undergo a chemical reaction with the coal species being dissolved. These solvents are the most common solvents used in high temperature direct liquefaction. The solvent reacts with the coal by donating hydrogen to the free radicals that are formed during the initial thermal degradation. Anthracene oil and tetralin are the chief examples of degrading and reactive solvents, respectively.

The amount of mineral matter present in a coal will have an effect on the conversion, but the effect may in fact be positive. The presence of metals in a coal sample (for example, $\mathrm{FeS}_{2}$ ) can actually have a catalytic effect on the process. This effect was studied by Mukherjee and Chowdry ${ }^{(43)}$ when they characterized the ash content of a high-vitrinite coal. They carried out liquefactions with this coal without using a donor solvent. However, specific minerals were added to the coal. The best catalytic activity, from their results, was obtained using added sulfur, both organic and pyritic. Other minerals that also showed an effect on liquefaction were iron, titanium, and kaolinite. In a separate study, conducted by Whitehurst et al ${ }^{(39)}$, the rate of solventsolvent hydrogen transfer reactions occurred at higher rates in the presence of coal which 
contained pyritic sulfur than in reactions with no coal present. The effect of mineral matter content in the feed coal on coal conversion can be seen in Figure $2.9^{(39)}$.

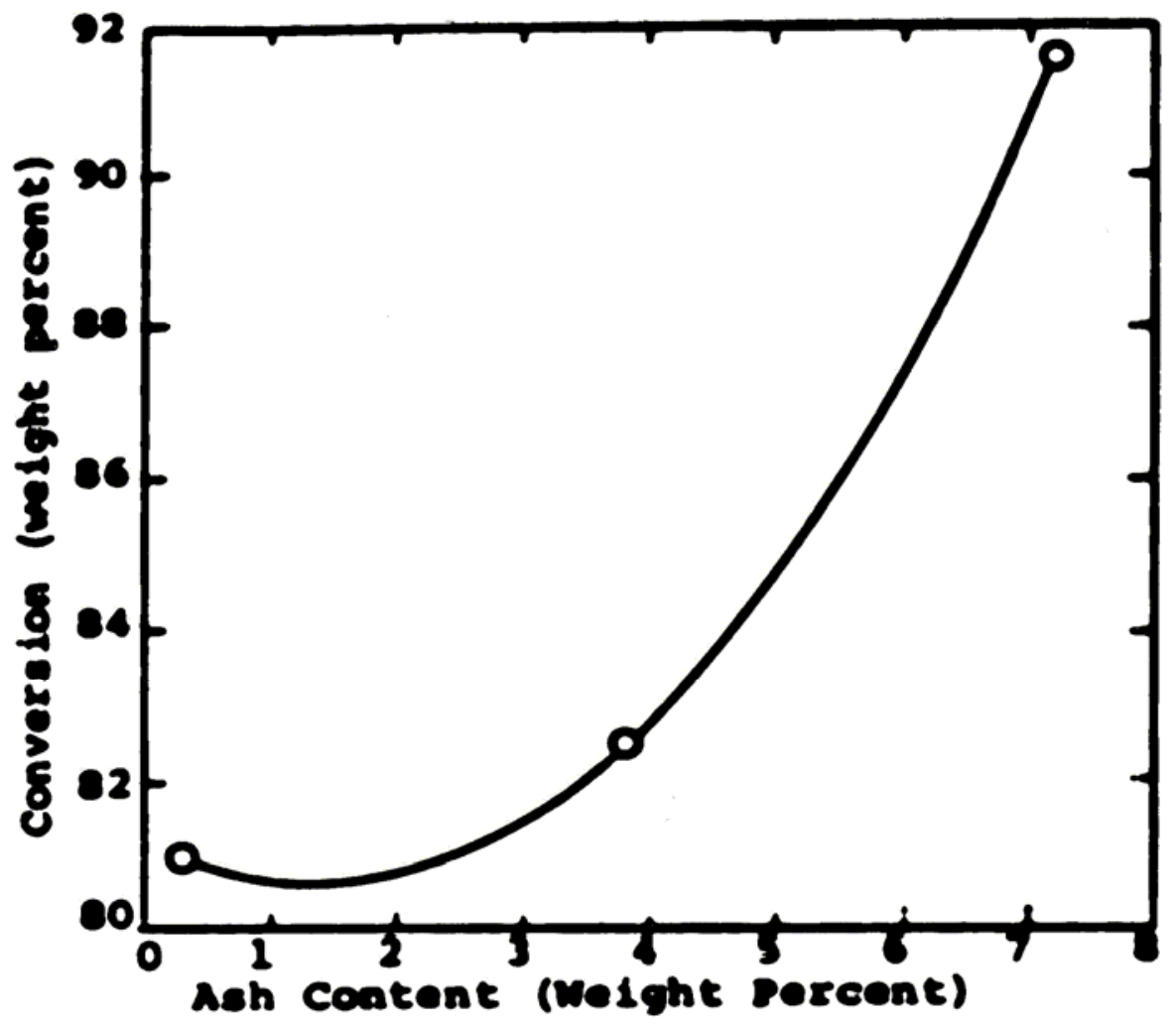

Figure 2.9 Wyodak Coal Conversion vs. Ash Content ${ }^{(39)}$

While the presence of a hydrogen atmosphere can increase the coal solubilization and conversion, the exact role the hydrogen gas has in liquefaction is not known specifically. Hydrogen gas could be used to stabilize free radicals either directly or by being transferred to the donor solvent, which then transfers the hydrogen to the radicals. It has been shown by Yen et al. ${ }^{(45)}$ that a hydrogen atmosphere has a positive effect on the coal conversion observed. The tests used tetralin as a donor solvent, and the benzene 
insolubles decreased from $25.3 \%$ with a nitrogen atmosphere to $11.5 \%$ when hydrogen was used. This indicates that there is a greater conversion of the benzene soluble portion of the coal structures during the reaction. This work has been substantiated by Tomic and Schobert ${ }^{(46)}$ where they observed that the conversion increased when using a hydrogen atmosphere as opposed to an inert atmosphere. They theorized that this was due to the ability of the hydrogen to reduce the amount of retrograde reactions occurring at the higher temperatures.

Though the effect of hydrogen is observable, the exact mechanism by which hydrogen gas affects liquefaction is still uncertain. Finseth et al. ${ }^{(47)}$ state that the bulk of the hydrogen that is consumed during uncatalyzed solvent liquefaction above $400^{\circ} \mathrm{C}$ is consumed from generating other gases (i.e. methane), removing heteroatoms, and hydrogenolysis of the coal matrix. Chawla et al. ${ }^{(48)}$ propose that hydrogen is consumed from secondary reactions as asphaltenes and preasphaltenes are converted to oils and gases at long times (greater than 15 minutes). Another study by Wilson et al. ${ }^{(49)}$ reported that hydrogen is used for alkyl fission and hydrogenolysis, but not for hydrogenating aromatic rings.

\subsection{Issues with Processing Techniques}

The current technologies used to produce carbon products and their precursors, while established, are becoming more expensive and less effective. The gradual decline in petroleum quality and rising prices of petroleum products will necessitate a switch to coal as the chief feedstock in carbon products. Coal processing itself has issues that must 
be resolved for coal to be effectively used as a carbon product feedstock. Coal, of course, is a very heterogeneous material, and the unwanted matter (inorganics, unconvertible matter) must be dealt with for coal to be used efficiently. The current technologies that are employed to process coal have difficulties that must also be overcome. Coal tar produced from carbonization must be further treated, and has many environmental issues related to it that prohibit the industry from becoming the chief supplier of pitches. Indirect liquefaction could produce very tailorable products, but the process is energy intensive and it completely breaks down the coal structure, which can be detrimental to a carbon pitch or coke, thus the process is seen as unreasonable for use in producing carbon products.

Direct liquefaction can be a process most amenable for carbon products, but the solvents used are typically expensive. These solvents, due to the high expense, must be recovered in any process which employs them. For example, in the laboratory it is difficult to recover all of the NMP from the solvent extraction process, but recovery of most is possible. However, tetralin recovery is a problematic process, mostly due to the fact that some of the tetralin used in a direct liquefaction process is converted to naphthalene. Reuse of this naphthalene would require first recovery and rehydrogenation of the naphthalene, which is costly. This defeats the purpose of hydrogenating the coal, as now the donor solvent requires hydrogenation to be reused. Naphthalene recovery can also be problematic, due to the very limited temperature range over which the chemical will remain a stable liquid in vacuum distillation (the typical laboratory scale recovery method). All of this points towards the need for a better solvent 
which is more easily recovered, is less expensive or, alternatively, a solvent which does not require any recovery at all.

\subsection{Potential Solutions}

A possible solution to the problem of using expensive extraction/liquefaction solvents that involve complicated and inefficient solvent recovery is the co-processing of coal and an industrial by-product solvent. There has been a large amount of work on coprocessing coal and industrial by-products, but most of the focus has been on producing liquid fuels. Additionally, the hydrogen donation ability of a given solvent can be easily enhanced by pre-treating via hydrogenation, and while the introduction of hydrogen gas in any process can be costly, the impact would be minimized as only mild hydrogenations of the solvent would be necessary to improve the conversion yield for a given coal.

\subsubsection{Coal / Solvent Co-Processing}

Co-processing is simply the processing of two separate materials together to upgrade them both. In much of the literature, coal is co-processed with an industrial byproduct solvent and the resultant material is more valuable material than either of the starting materials. Occasionally the coal is co-processed with recycled polymers, but this is not applicable to the present research. Most often, though, this is done with either heavy oils of petroleum origin or coal derived heavy oils. The technology is investigated as a method to develop synthetic fuels to bridge the gap between the current petroleum based technology and a possible future coal based fuel technology. The idea is to use the 
petroleum residuum as the solvent / slurrying agent and the coal as a hydrocarbonaceous feedstock ${ }^{(50)}$.

As early as 1935 , Boomer and Saddington ${ }^{(51)}$ pioneered the testing of such a concept. They used crude Athabasca tar sand bitumen for the conversion of Alberta subbituminous and bituminous coals. The oils and coals were combined and heated together at $425-450^{\circ} \mathrm{C}$ for 3 to 8 hours under a hydrogen atmosphere, whereby the conversion of coal to liquid and gaseous products exceeded $50 \%$. The solvolysis process, for the dissolution of coal in asphalt or residual oils, is under development in Japanese laboratories. Here, one part coal is dissolved in 3 parts asphalt at $390^{\circ} \mathrm{C}$ and $100 \mathrm{kPa}$ hydrogen or nitrogen. These conditions are optimal for the operation of the first stage of their liquid fuels process since they minimize the formation of coke by the "polycondensation of asphaltenes" and the formation of gases ${ }^{(52)}$. The solvolysis pitch, or the effluent from this first-stage, after solids removal, can then be hydrocracked over a catalyst to obtain liquid fuels.

Much of the work done is in the area of utilizing by-products or waste streams as liquefaction solvents. Waste oils such as vacuum pyrolyzed tire oils, waste automobile crankcase oil and vacuum pyrolyzed plastic oils are used in the study by Orr, et al. ${ }^{(53)}$. The results of their work showed that the vacuum pyrolyzed tire oil gave the best overall conversion under the conditions examined. They attributed the high conversion to the presence of polyaromatic non-donor molecules present in the oil and the high pyrite content of the coal used in the study (Table 2.6) 
Table 2.6 Total conversion for Illinois No. 6 coal and oil solvents at $430^{\circ} \mathrm{C}$ in tubing reactors for 1 hour under 1000 psig $\mathrm{H}_{2}$ (cold) ${ }^{(53)}$

\begin{tabular}{|r|ccc|}
\hline & $\begin{array}{c}\text { Automotive } \\
\text { Crankcase Oil }\end{array}$ & $\begin{array}{c}\text { Vacuum Pyrolyzed } \\
\text { Plastic Oil }\end{array}$ & $\begin{array}{c}\text { Vacuum Pyrolyzed } \\
\text { Tire Oil }\end{array}$ \\
\hline Gas + Oil & 66.1 & 64.4 & 65.8 \\
Asphaltenes & 10.4 & 7.2 & 17.8 \\
Total Conversion & 76.5 & 71.6 & 83.6 \\
\hline
\end{tabular}

Other examples exist in the literature, including liquefying coal by extraction with a petroleum pitch that has been hydrogenated at high pressure over a Ni/Mo catalyst. Also, when small coal particles are added to residua and the slurry is heated up to $420^{\circ} \mathrm{C}$ for 3 hours, liquid fuels are produced. The COIL (catalytic hydrocracking of coal-oil mixtures) process for the catalytic hydrocracking of coal-oil mixtures was being developed at Hydrocarbon Research, Inc. (HRI). This process was a follow up to the HCOAL process for coal liquefaction and the H-OIL process for heavy oil hydrocracking. Other workers have investigated the ebullated bed processing of a mixture of 1 part coal and 1-2 parts vacuum residual oil at $450^{\circ} \mathrm{C}$ and a hydrogen partial pressure of $14 \mathrm{MPa}$ in the presence of a Co/Mo catalyst ${ }^{(51)}$. In studies by Wallace et al. ${ }^{(54)}$, heavy petroleum residues were used as co-processing solvents, typically with bi-metallic catalysts. Yoshinari Inukai found that there is a synergistic effect when coal and petroleum atmospheric residue are co-processed ${ }^{(55)}$.

Generally, the use of this coal-oil co-processing, as mentioned previously, results in a technological and economical advantage over the conventional liquefaction processes. Still, most of the work has been in the area of producing synthetic fuels or fuel precursors. To use this in the production of a carbon product precursor, the 
conditions should be much milder, which increases the economic advantages in the technology. One aspect of the co-processing that would carry over into this research is the upgrading of the solvent prior to use in the reaction via hydrogenation. While the basis of this research is the direct liquefaction process, and while the practice of coprocessing is used in this thesis, the resultant process is neither true liquefaction nor a typical co-processing scheme. Thus from this point forward the process will simply be called a digestion, i.e. the coal is digested in an industrial by-product solvent to produce pitch as coker feeds.

\subsubsection{Solvent Pre-Hydrogenation}

The idea of using industrial by-product solvents in the treatment of coal is not a new one. Much research has been conducted on using these solvent as a processing medium for the production of fuels from coal. Since transportation fuels are the intended products in these studies, these processes are much more severe in nature than what would be required for the use in producing carbon products, such as coke feeds or binder pitches.

During coal digestion in aromatic solvents, prior research suggests that the destruction of the coal structure occurs in two stages. First, the alkyl chains and crosslinks in the coal are thermally cleaved. This is then followed by the transfer of hydrogen from the solvent, which stabilizes the radicals formed and prevents retrograde reactions

from occurring ${ }^{(56)}$. These industrial by-product solvents must be compatible with the essentially aromatic species generated from the coal to retain the fragments in solution. Some of the most promising petroleum derived solvents are, conveniently, derived from 
the less valuable heavy ends of the refinery streams. Solvents recovered from by-product coking ovens are also very good candidates for use as liquefaction solvents.

Still, the effectiveness of the solvents themselves can be limited, depending on the availability of transferable hydrogen. While digestion can be carried out in a hydrogen atmosphere, it is more cost effective to limit the use of hydrogen gas in a digestion process. The design of a process could be such that hydrogen gas is only used in the pretreatment of the solvent to be used in the process.

The idea of pretreating solvents is substantiated in the literature, such as in the research conducted by Kouzu, et al. ${ }^{(57)}$. They found that there was a clear increase in the hexane soluble fraction with an increase in the hydroaromatic content of the solvent. The NEDOL process (Figure 2.10), a coal liquefaction process developed by the New Energy and Industrial Technology Development Organization (NEDO) in Japan, is run in a 150 t/d pilot plant. Coals are directly converted into oils in the presence of a hydrogen donor solvent and iron catalyst under hydrogen pressure. The oils are divided into three fractions, and the heavy fraction is recycled as the hydrogen donor solvent after hydrotreatment. This heavy oil fraction contains a large amount of aromatics such as naphthalene, phenanthrene and pyrene. These aromatics, however, do not possess any hydrogen-donating ability, so they are hydrogenated into hydroaromatics.

In this particular study, a sub-bituminous coal was processed (75.0\% C and 5.8\% $\mathrm{H}$, daf). The prepared solvents used in this study were hydrotreated in a fixed bed reactor over a Ni/Mo catalyst. The results show an increase in the naphthenic hydrogen content of the solvent with increasing hydrogen gas consumption in the hydrotreatment stage (Figure 2.11). This indicates that aromatic hydrogenation occurred in this stage. They 
also found that as the content of naphthenic hydrogen increased in the hydrotreatment stage, the amount of hydrogen utilized in the liquefaction stage increased. The yield of oils increased by nearly 4wt\%-daf with an increase in hydrogen gas consumption of about 0.6wt\%-daf in the hydrotreatment stage. Simultaneously, the yield of liquefaction residue decreased by almost $7 \mathrm{wt} \%$-daf and that of the organic gases increased almost 3 wt\%-daf. In addition to this, the increased hydrogenation of the solvent results in a lower concentration of nitrogen in the oil fraction after the liquefaction. Kouzu and his research associates also found that the naphthenic hydrogen in the solvent acts more efficiently than the gaseous hydrogen for the formation of the oil fraction in the liquefaction stage, as well as the reduction of asphaltenes. These results are consistent with results reported with using model compounds. 


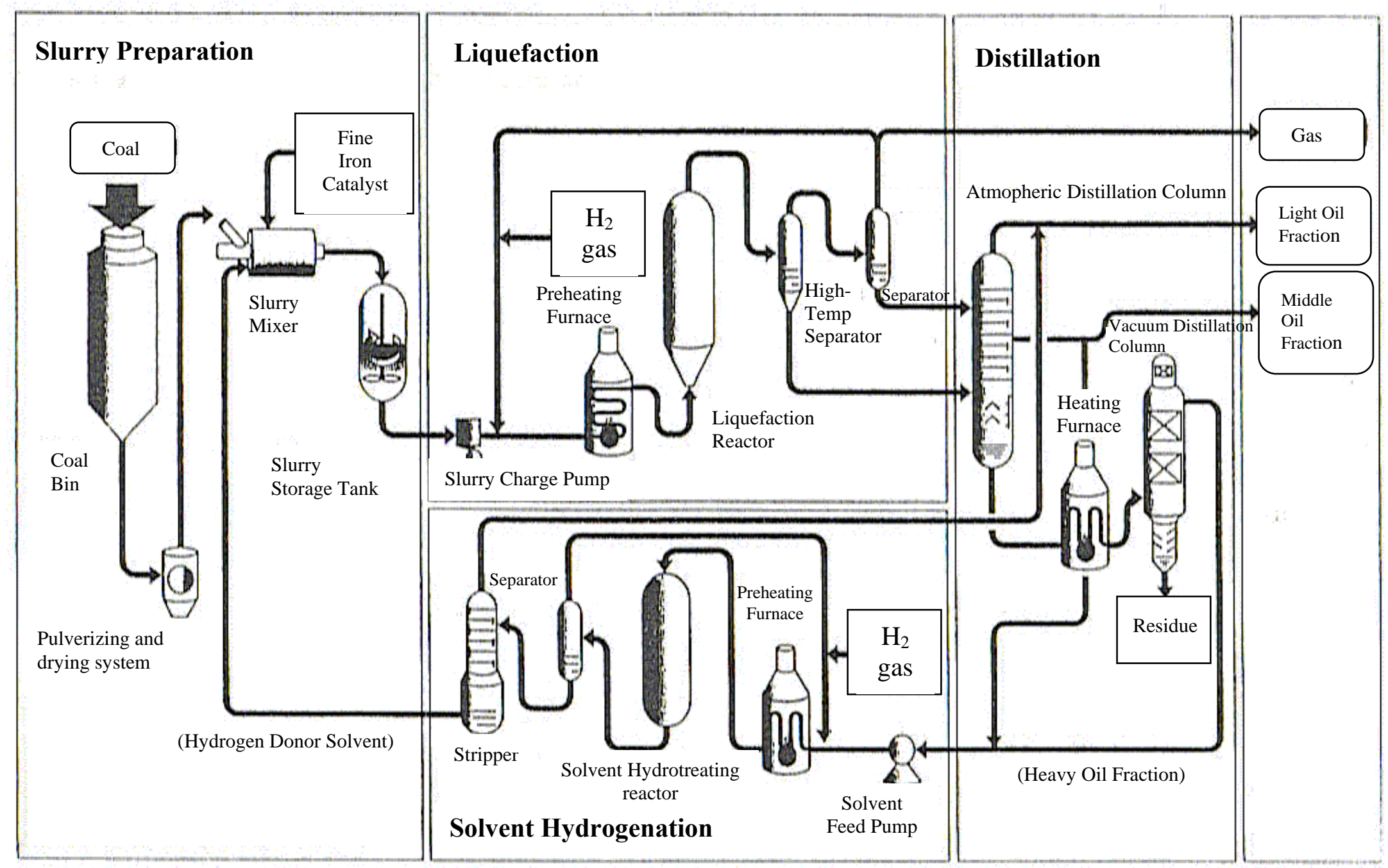

Figure 2.10 Schematic flow diagram of the NEDOL 150ton/day pilot plant ${ }^{(57)}$ 


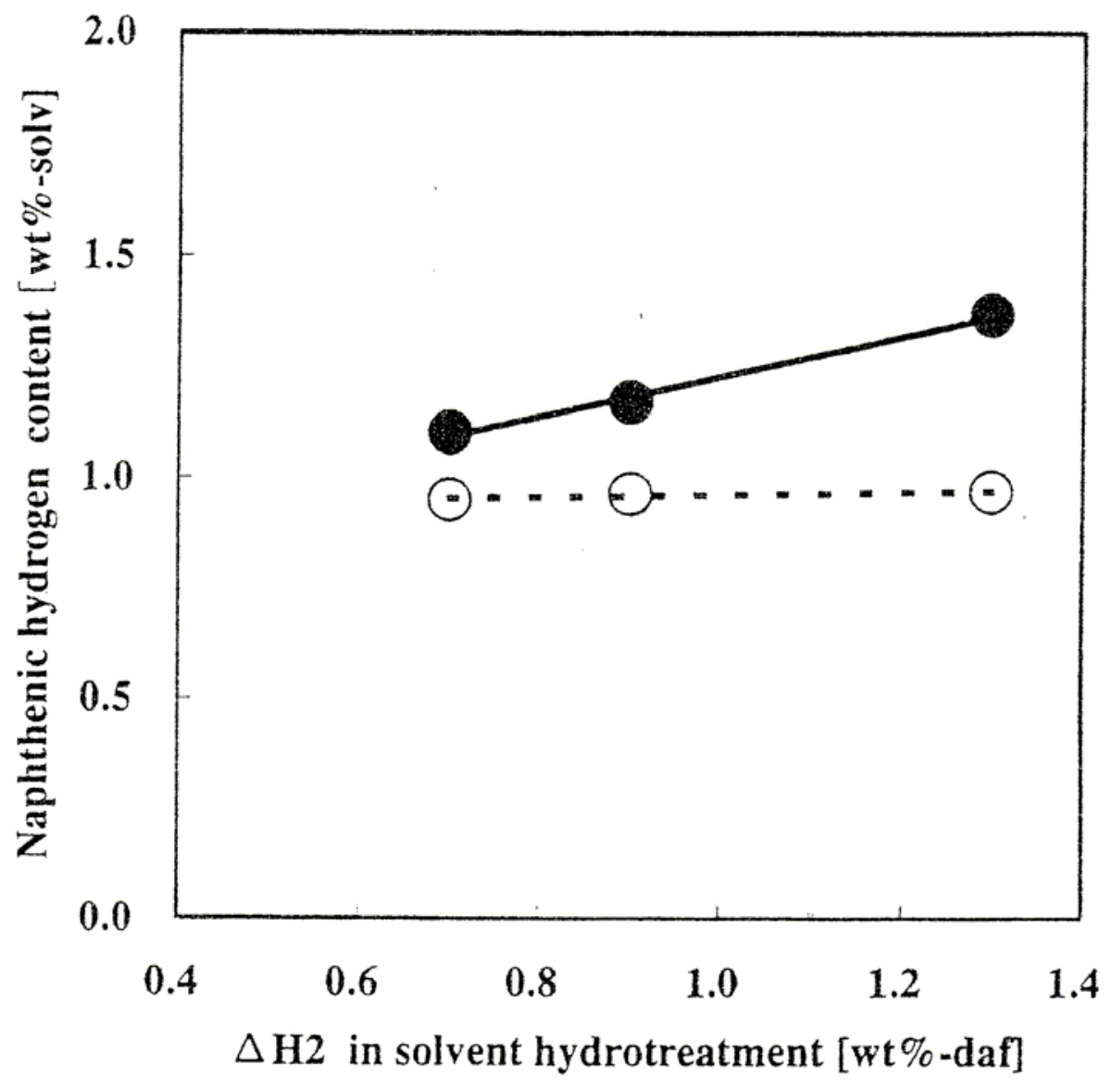

Figure 2.11 Variation of naphthenic $\mathrm{H}_{2}$ contents in feed ( $(0)$ and hydrogenated solvent $(\bullet)$ as a function of $\mathrm{H}_{2}$ gas consumption in solvent treatment ${ }^{(57)}$

In a study by Sato et al. ${ }^{(58)}$, the properties of solvents commonly used in liquefaction research were examined before and after hydrogenation. The amount of hexane insoluble material in a coal liquid, shale oil, tar sand bitumen and anthracene oil were essentially eliminated with hydrogenation, and the vacuum distillation bottoms decreased, usually by as much as $50 \%$. The one exception was the anthracene oil, which did show a slight increase in vacuum distillation bottoms with hydrogenation (Table 2.7). After the solvents were characterized, they were used as liquefaction solvents with a Japanese sub-bituminous coal. Their results show that oil yields increased with 
hydrotreatment of the solvents, particularly with the tar sand bitumen after severe hydrotreatment. Their results showed that while conversion could be increased with hydrotreatment of the shale oil and tar sand bitumens, these solvents are not good solvents without pretreatment. This is mostly a result of their poor affinity for coal due to their paraffinic character, which would subsequently give rise to retrogressive reactions with the coal fragments. The anthracene oil, as received and hydrogenated, was the better of the solvents tested, in spite of it higher $\mathrm{C} / \mathrm{H}$ ratio.

Table 2.7 Properties of Heavy Solvents ${ }^{\text {a }(58)}$

\begin{tabular}{|c|c|c|c|c|}
\hline \multirow{2}{*}{$\begin{array}{l}\text { Sample } \\
\text { Name }\end{array}$} & \multicolumn{2}{|c|}{ Hexane Extraction } & \multicolumn{2}{|c|}{ Vacuum Distillation $^{\text {b }}$} \\
\hline & Soluble & Insoluble & Distillate & Bottom \\
\hline \multicolumn{5}{|l|}{ Coal Liquid } \\
\hline \multirow{2}{*}{ Hydrotreated @ $\begin{array}{r}\text { Neat } \\
390^{\circ} \mathrm{C}\end{array}$} & 98.8 & 1.20 & 95.66 & 4.34 \\
\hline & 100.0 & - & 97.64 & 2.36 \\
\hline \multicolumn{5}{|l|}{ Shale Oil } \\
\hline \multirow{3}{*}{$\begin{array}{l}\text { Neat } \\
\text { Hydrotreated @ } 390^{\circ} \mathrm{C} \\
\text { Hydrotreated @ } 420^{\circ} \mathrm{C}\end{array}$} & 98.8 & 1.20 & 75.36 & 24.64 \\
\hline & 100.0 & - & 85.70 & 14.30 \\
\hline & 100.0 & - & 89.90 & 10.10 \\
\hline \multicolumn{5}{|l|}{ Tar Sand Bitumen } \\
\hline Neat & 85.0 & 15.0 & 37.89 & 62.11 \\
\hline \multirow{2}{*}{$\begin{array}{l}\text { Hydrotreated @ } 390^{\circ} \mathrm{C} \\
\text { Hydrotreated } @ 420^{\circ} \mathrm{C}\end{array}$} & 100.0 & - & 52.45 & 47.55 \\
\hline & 100.0 & - & 76.91 & 23.09 \\
\hline \multicolumn{5}{|l|}{ Anthracene Oil } \\
\hline Neat & 99.7 & 0.3 & 98.51 & 1.49 \\
\hline Hydrotreated @ $390^{\circ} \mathrm{C}$ & 100.0 & - & 98.29 & 1.71 \\
\hline Hydrotreated @ $420^{\circ} \mathrm{C}$ & 100.0 & - & 97.65 & 2.35 \\
\hline
\end{tabular}

Other research substantiates these findings. Work conducted by Miranda et al. ${ }^{(59)}$ on the hydrogenation of a Spanish coal indicated that the best results can be obtained with hydrogenated anthracene oil at low temperatures and reaction times. Caballero et al. ${ }^{(60)}$ also found that hydrotreating anthracene oil prior to its use as a liquefaction solvent 
greatly enhanced the coal conversions obtained. In Figure 2.12, the difference in liquefaction yields using fresh anthracene oil and hydrogenated anthracene oil are very apparent. However, it should be noted that at high enough temperatures, even the solvent can be converted to chars via retrogressive reactions with the coal. The conversions diminish to near zero at a temperature of $475^{\circ} \mathrm{C}$, but once the solvent is prehydrogenated, conversions are obtainable even at these more extreme temperatures. This is most likely due to a greater $\mathrm{H}$-donor capacity now present in the treated solvent. Yoshinari Inukai found that hydrogenated petroleum atmospheric residue (HAR) gives better coal conversion than the as received residue, while solvent heat-treatment alone in fact causes the residue to give lower coal conversions ${ }^{(55)}$.
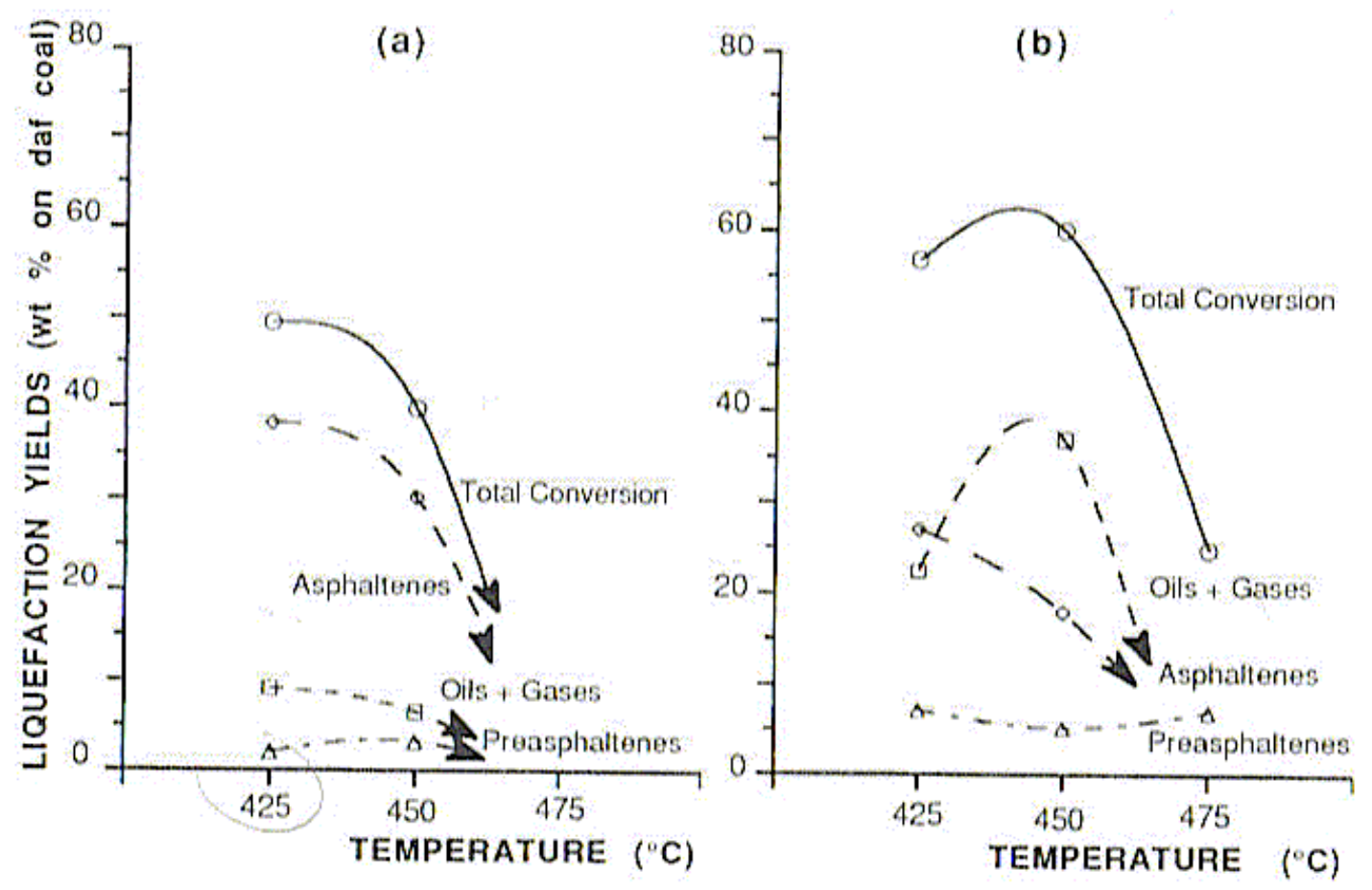

Figure 2.12 Influence of Solvent Pretreatment: (a) Fresh Anthracene Oil; (b) Hydrogenated Anthracene Oil ${ }^{(60)}$ 
The upgrading of coal-derived and petroleum-derived liquids for use as liquefaction solvents is typically done with a catalyst present. Much research has been conducted on the types of catalyst to be used. In a study by Yanlong Shi, William F. Olson and Edward M. Eyring ${ }^{(61)}$, vacuum pyrolyzed tire oil (VPTO) was partially prehydrogenated (HVPTO) for use as a co-processing solvent. The solvent hydrogenation was done using several types of pre-sulfided hydrogenation catalysts, including $\mathrm{Ni} / \mathrm{Al}_{2} \mathrm{O}_{3}$, $\mathrm{CoMo} / \mathrm{Al}_{2} \mathrm{O}_{3}, \mathrm{NiMo} / \mathrm{Al}_{2} \mathrm{O}_{3}, \mathrm{NiW} / \mathrm{Al}_{2} \mathrm{O}_{3}, \mathrm{Ni} / \mathrm{SiO}_{2}-\mathrm{Al}_{2} \mathrm{O}_{3}, \mathrm{Pd} /$ carbon, unsulfided Pt/ $\mathrm{Al}_{2} \mathrm{O}_{3}$, and Pt/carbon. The solvents were hydrogenated at $325^{\circ} \mathrm{C}$ and $1000 \mathrm{psig} \mathrm{H}_{2}$ (cold) for 1 hour. Their results indicated that the $\mathrm{NiMo} / \mathrm{Al}_{2} \mathrm{O}_{3}\left(6.7 \%\right.$ of $\mathrm{NiO}$ and $27 \% \mathrm{MoO}_{3}$ ) and $\mathrm{Ni} / \mathrm{Al}_{2} \mathrm{O}_{3}$ were the best catalysts for converting the polyaromatics to hydrogen donor-rich hydroaromatics (Table 2.8). The best conversions were compared to those obtained using model compounds such as tetralin, anthracene, 9,10-dihydroanthracene and phenanthrene, and the tire oil compared very well once hydrogenated. 
Table 2.8 - Effect of using hydrogenation catalysts in preparing HVPTO ${ }^{(61)}$

\begin{tabular}{|l|c|}
\hline \multicolumn{1}{|c|}{ Hydrogenation Catalyst } & $\begin{array}{c}\text { Total Conversion } \\
\text { (\%) for maf Coal }\end{array}$ \\
\hline $\mathrm{Ni} / \mathrm{Al}_{2} \mathrm{O}_{3}{ }^{\mathrm{a}}$ & 63.8 \\
$\mathrm{CoMo} / \mathrm{Al}_{2} \mathrm{O}_{3}{ }^{\mathrm{a}}$ & 59.8 \\
$\mathrm{NiW} / \mathrm{Al}_{2} \mathrm{O}_{3}{ }^{\mathrm{a}}$ & 61.6 \\
$\mathrm{NiMo} / \mathrm{Al}_{2} \mathrm{O}_{3}\left(6.7 \% \mathrm{NiO}, 27 \% \mathrm{MoO}_{3}\right)^{\mathrm{a}}$ & 67.1 \\
$\mathrm{NiMo} / \mathrm{Al}_{2} \mathrm{O}_{3}\left(5.4 \% \mathrm{NiO}, 20 \% \mathrm{MoO}_{3}\right)^{\mathrm{a}}$ & 57.2 \\
$\mathrm{Pt} / \mathrm{Al}_{2} \mathrm{O}_{3}$ & 55.7 \\
$\mathrm{Pt} /$ carbon & 61.1 \\
$\mathrm{Pd} /$ carbon & 50.3 \\
$\mathrm{Ni} / \mathrm{Al} \mathrm{O}_{3}-\mathrm{SiO}{ }_{2}{ }^{\mathrm{a}}$ & 50.2 \\
$\mathrm{No}$ catalyst & 34.1 \\
\hline
\end{tabular}

a - presulfided at $350^{\circ} \mathrm{C}$ for $2 \mathrm{hrs}$

The study of hydroliquefaction of Illinois No. 6 coal with petroleum atmospheric residue ${ }^{(55)}$ found that the best conversion occurs using an oil-soluble Mo catalyst. Even pre-hydrogenated residue worked better with the oil-soluble catalyst. This is no doubt due to the higher accessibility of the catalyst to the solvent, as compared to the granular catalyst. Other work has been done on using a variety of catalysts on heavy oils and distillates in preparation for using them as liquefaction solvents. Townsend and Larkins have an extensive study on the upgrading of Australian coal-derived liquids for use in liquefaction processing ${ }^{(62)}$. Their results cover the physical properties of the catalysts and how these affect their efficiency, including the catalyst porosity, surface area, as well as the support type, catalyst acidity and type of metal. While this work is exceptional in its meticulousness and scope, it is beyond the scope of this study. 
Care must be taken when treating the solvents against over-hydrogenation, which can result in the production of alicyclics that are poor H-donors in comparison to hydroaromatics ${ }^{(63)}$. When hydrogenating creosote oils or anthracene oils (both coalderived), it was observed that there was a maximum conversion obtained when plotted against the $\mathrm{H}$ n.m.r. aromaticity of the solvents (Figure 2.13). While this maximum was rather broad, it does speak to the necessity of controlling the amount of pretreatment required for the desired conversion of coal. As the level of pretreatment increased, the production of alicyclics increased, thereby reducing the overall H-donor ability of the solvent. The trends in this study are indicative of a sequential reaction scheme, shown below:

Polynuclear Aromatics $\rightarrow$ Two-Ring Aromatics $\rightarrow$ One-Ring Aromatics $\rightarrow$ Saturates 

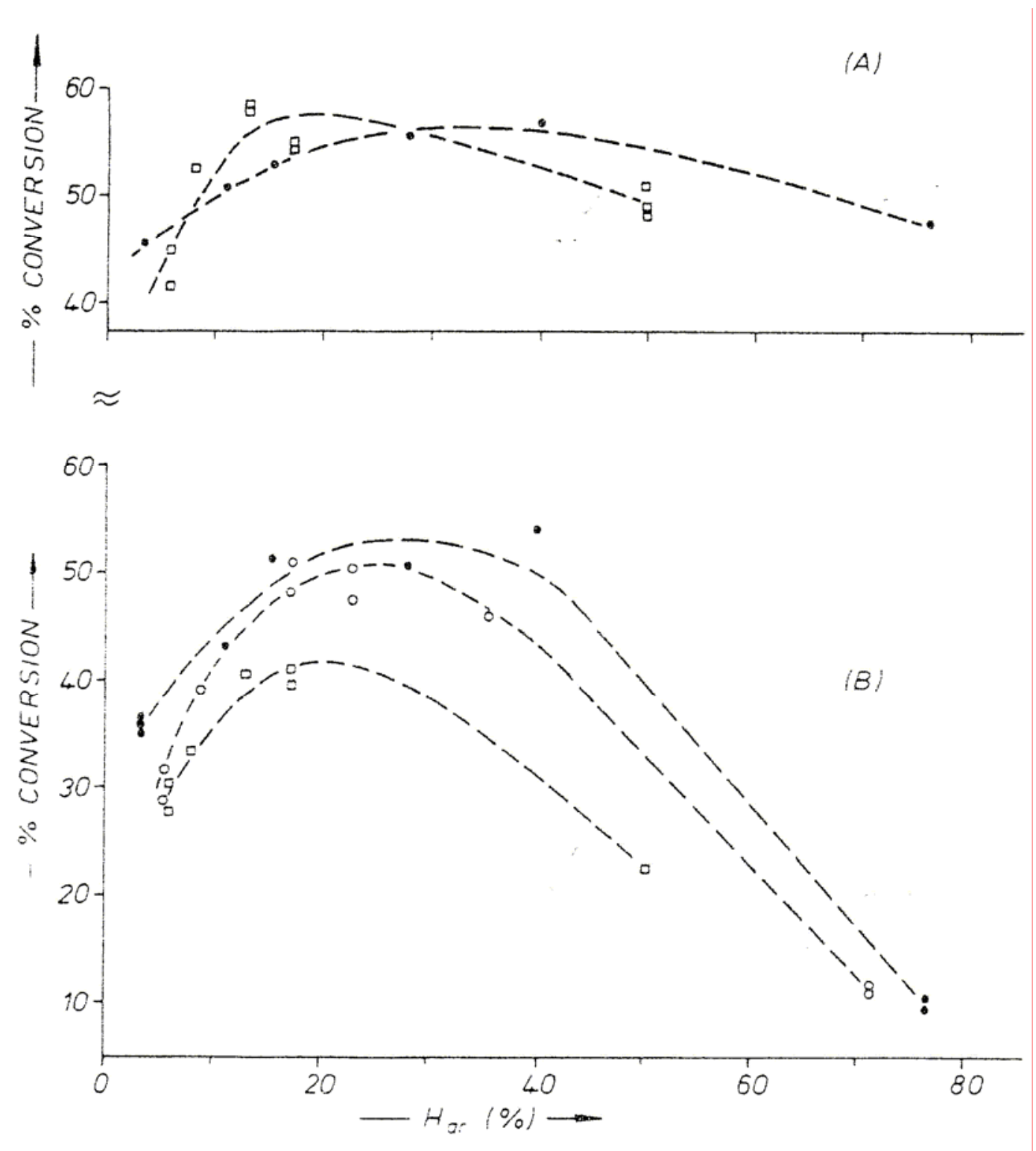

Figure 2.13 Conversion as a function of $\mathrm{H}$ n.m.r. aromaticity, $\mathrm{H}_{\mathrm{ar}}$, of hydrogenated solvents, using $\mathrm{H}_{2}$ gas (A) and $\mathrm{N}_{2}$ gas (B) atmospheres ${ }^{(63)}$

\subsection{Summary of Background Remarks}

All of this research points towards the viability of using an industrial by-product or waste stream as the solvent material for a "co-processing” digestion with coal. These solvents' H-donor abilities can be greatly enhanced using catalytic hydrogenation prior to 
the digestion step. As liquid fuels are not the desired product in this thesis, the hydrogenation and digestion steps would be mild comparatively, which lowers potential cost and safety issues. The final product would then be treated to turn it into a pitch, which would then be used as a potential coker feed or binder pitch in the production of a carbon anode for use by the aluminum industry. The physical and chemical characteristics of the coal and solvents would contribute to the final characteristics of the pitch product, which could be tailored for a particular industry, using the air-blowing method discussed above. 


\section{CHAPTER 3 - EXPERIMENTAL PROCEDURES}

In this chapter, all of the materials and equipment used in the experiments are discussed, along with the processes of catalyst sulfiding, solvent hydrogenation, coal digestion, THF extraction of the products, followed by the scale-up process and the pitch modification (air-blowing), carbonization of the coal digests, as well as the analytical tests performed on the feeds and products. Process flow diagrams for both the small scale $\left(30 \mathrm{~cm}^{3}\right.$ ) and large scale (one gallon) experiments are given in Figures 3.1 and 3.2.

\subsection{Materials}

Kingwood Coal - The coal was obtained and ground as received from Kingwood Mining Co. Several large pans of the coal were dried overnight in a vacuum oven, set to a temperature of $100^{\circ} \mathrm{C}$, with a slow nitrogen purge and $25-30 \mathrm{~mm} \mathrm{Hg}$ vacuum. Once the coal had cooled, the vacuum was broken, and the coal was then placed into dark-glass containers and stored in a cold room to prevent oxidation.

Carbon Black Base (CBB) - Similar to Creosote Oil, CBB was received from Kopper's Industries in a 55-gallon barrel. The barrel was heated using electric heating bands. A motorized stirring mechanism was attached to the top of the barrel to mix the material so a uniform sample could be retrieved in two 5-gallon buckets. The oil became a paste at room temperature. Before a sample was taken from the 5-gallon containers, the CBB was warmed to about $100^{\circ} \mathrm{C}$ in an oven. The $\mathrm{CBB}$ was very fluid at this temperature, thus the taking of a representative sample after stirring was assured. 
RCC Slurry Oil (SO) - This was obtained from Marathon-Ashland Petroleum, LLC, specifically from their Findlay, $\mathrm{OH}$ facility. Initially a small 1-gallon sample for testing was acquired. Later, more solvent was shipped from Marathon-Ashland Petroleum, LLC in two 5-gallon buckets.

Heavy Oil (Anthracene Oil - AO) - This solvent was received from Reilley Industries in a 5-gallon bucket. The sample obtained appeared to have some suspended solids material that did not melt even after heating to $100^{\circ} \mathrm{C}$. A heated filtration was performed, and the solids content was determined to be $1.60 \%$.

Maraflex 1000 Oil (MO) - Also received from Marathon-Ashland Petroleum, LLC in Findlay, OH. This oil is a lighter type of oil, which resembles clean engine oil.

1,2,3,4 Tetrahydronaphthalene (Tetralin) - Acquired from Aldrich Chemical Company. It was used as received with $97 \%$ purity. This solvent is the standard coal extraction / hydrogenation medium, used in this work as a control solvent.

Tetrahydrofuran (THF) - HPLC grade, acquired from Fisher Scientific. This solvent was used in all of the product extractions to determine the digestion efficiency.

Di-methyl Sulfoxide (DMSO) - Acquired from Fisher Scientific, certified ACS. This solvent was used as the sulfiding agent for the activation of the catalyst.

Carbon Di-sulfide ( $\left.\mathbf{C S}_{2}\right)$ - Acquired from Fisher Scientific. This solvent was used in the Simulated Distillation Chromatography analysis.

Toluene - Acquired from Fisher Scientific. This solvent was used in the determination of the solubilities of the digests and air-blown digests.

Catalyst - The catalyst chosen for these solvent hydrogenations was made available through a generous donation from the Criterion Catalyst and Technologies Corporation. 
Their 424 catalyst is a Ni/Mo catalyst on an alumina support and is used for heavy oil hydrotreaters at severe conditions (i.e. first stage hydrotreater, VGO hydrotreater, or lube oil hydrotreater). The catalyst is of proven high hydrogenation activity and was judged suitable for upgrading heavy, aromatic oils.

Gases - Hydrogen was used as the gaseous atmosphere in the solvent hydrogenations, and nitrogen was used as an inert gaseous blanket in the coal digestions. Compressed air was used in the coke precursor modification. All gases were obtained as laboratory standard grade from AirGas of West Virginia.

The characteristics of the Kingwood coal are given below in Table 3.1, and the elemental analyses of the four raw solvents and the coal sample are given in Table 3.2. 
Table 3.1 - Characteristics of Kingwood Coal

\begin{tabular}{|c|c|}
\hline Coal Bed & Kingwood \\
\hline Seam & Kittaning \\
\hline County & Preston \\
\hline State & WV \\
\hline ASTM Rank & High Volatile Bituminous \\
\hline \% Volatile Matter (dry) & 33.17 \\
\hline \% Ash (dry) & 8.92 \\
\hline \% Fixed Carbon (dry) & 57.91 \\
\hline \% Sulfur (dry) & 1.84 \\
\hline NMP Extraction Yield, \% & 66.70 \\
\hline Mean-Max reflectance of vitrinite & 1.08 \\
\hline Total Vitrinite & 74.60 \\
\hline Total Liptinite & 5.00 \\
\hline Total Inertinite & 19.40 \\
\hline
\end{tabular}

Table 3.2 - Elemental Analysis Results for Coal and Four (4) Candidate Solvents

\begin{tabular}{|c|c|c|c|c|c|}
\hline & $\begin{array}{c}\text { Kingwood } \\
\text { Coal }\end{array}$ & $\begin{array}{c}\text { Carbon } \\
\text { Black Base } \\
\text { (CBB) }\end{array}$ & $\begin{array}{c}\text { RCC } \\
\text { Slurry Oil } \\
\text { (SO) }\end{array}$ & $\begin{array}{c}\text { Maraflex } \\
\mathbf{1 0 0 0} \text { Oil } \\
\text { (MO) }\end{array}$ & $\begin{array}{c}\text { Riley's } \\
\text { Heavy Oil } \\
\text { (AO) }\end{array}$ \\
\hline $\mathbf{C}$ & 77.44 & 91.58 & 87.38 & 92.22 & 92.41 \\
\hline $\mathbf{H}$ & 4.95 & 5.71 & 9.56 & 7.77 & 6.21 \\
\hline $\mathbf{N}$ & 1.18 & 1.09 & 0.44 & 0.37 & 0.94 \\
\hline $\mathbf{S}$ & 1.58 & 0.48 & 2.62 & 0.96 & 0.27 \\
\hline $\mathbf{O}$ & 5.93 & 1.15 & - & - & 0.17 \\
\hline $\begin{array}{c}\text { C/H Atomic } \\
\text { Ratio }\end{array}$ & 1.52 & 1.34 & 0.76 & 0.99 & 1.24 \\
\hline
\end{tabular}




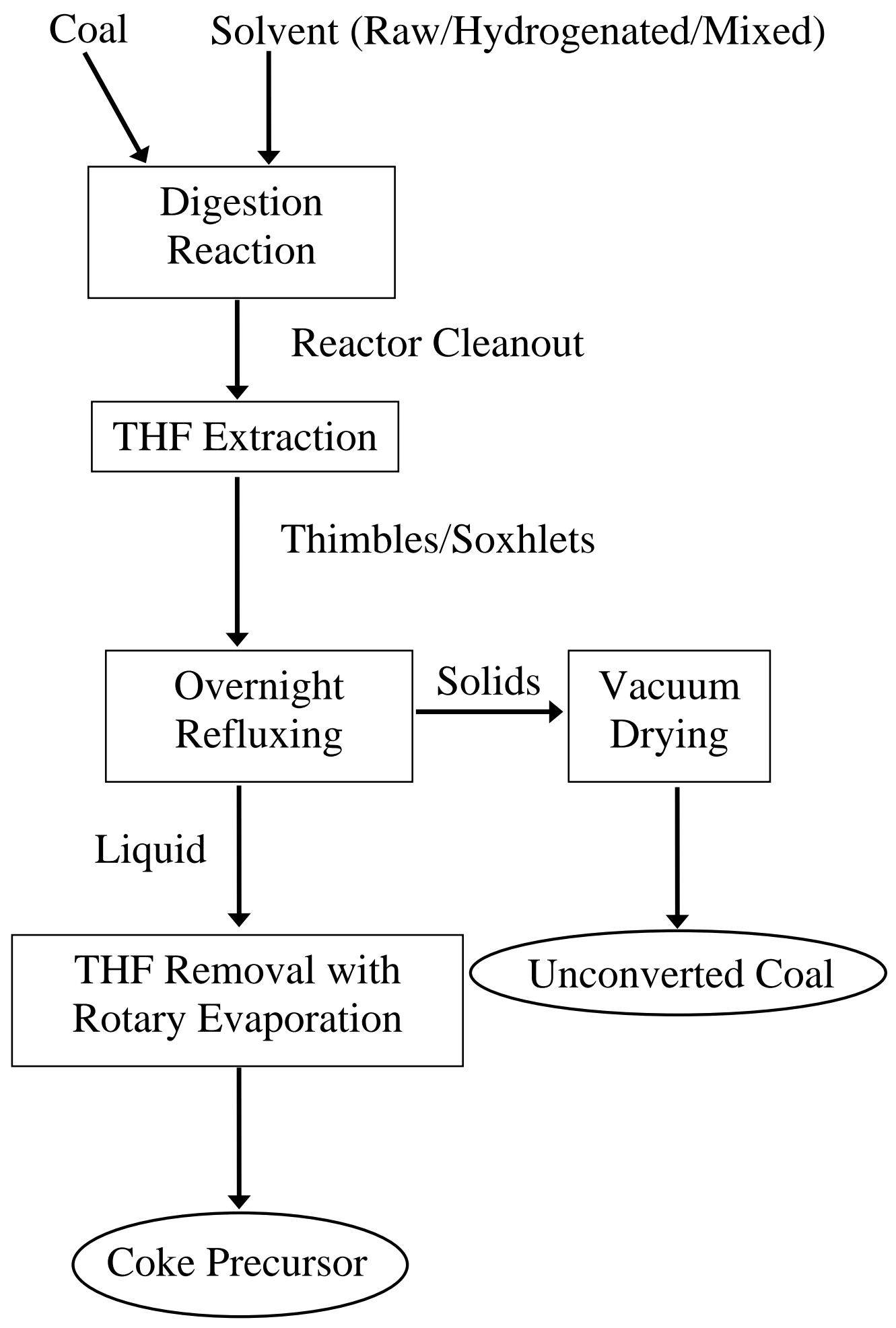

Figure 3.1 - Process Flow Diagram for Coal Digestion (small scale) 


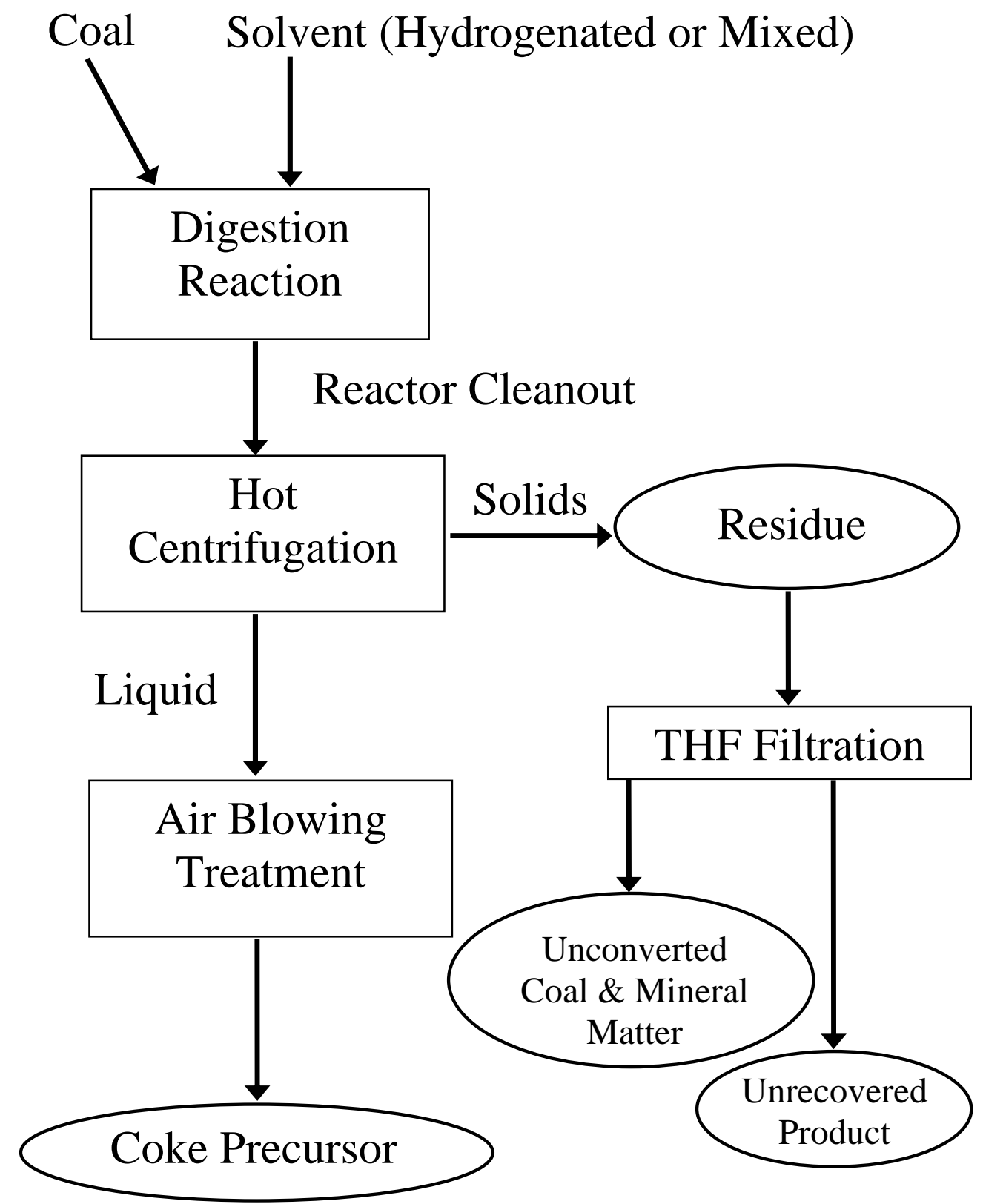

Figure 3.2 - Process Flow Diagram for Coal Digestion (1-gallon) 


\subsection{Catalyst Pretreatment}

\subsubsection{Catalyst Basket}

The Ni/Mo catalyst is typically used in plug-flow reactors, but there were only stirred tank reactors available for this research, hence some support structure needed to be fashioned. The stirred tank reactor used in this study had a volume of five gallons, but it was decided that only half of the volume would be used, so as not to produce large quantities of potentially unusable solvents. Using this volume and the dimensions of the reactor, the estimated liquid level for the solvent hydrogenation runs was calculated to be approximately 9.1 inches. Then, using the bulk density of the catalyst particles (see Table 3.3) and a reactant volume to catalyst volume ratio of 7 to 1 , the size of an annular basket was calculated (see Table 3.4). The 1/20” size catalyst was used.

The basket was constructed from 316 stainless steel materials purchased from McMaster-Carr. The basket was constructed in such a way to allow for solvent to flow through the screen, which held the catalyst particles securely. The solvent flows from inside to outside of the basket, with the reactor agitator set in the middle of the basket. The basket was propped up on legs to allow the solvent to flow under the basket, and the basket was short enough to allow for solvent to flow over the top of the basket when the agitator was on (see Figure 3.3). The finished basket fit into the reactor very snuggly, and allowed for the reactor thermowell to lower into the reactor properly (See Figure 3.4). 
Table 3.3 - Catalyst Properties

\begin{tabular}{|c|c|c|}
\hline \multicolumn{3}{|c|}{ TYPICAL PROPERTIES } \\
\hline Shape & TRILOBE & TRILOBE \\
\hline Nominal Size, mm (in.) & $1.3(1 / 20)$ & $1.6(1 / 16)$ \\
\hline Chemical Composition, wt\% dry basis & & \\
\hline Nickel & 3.0 & 3.0 \\
\hline Molybdenum & 13.0 & 13.0 \\
\hline Physical Properties & & \\
\hline Surgace Area, $\mathrm{m}^{2} / \mathrm{g}$ & 155 & 155 \\
\hline Pore Volume, cc/g $\left(\mathrm{H}_{2} \mathrm{O}\right)$ & 0.45 & 0.45 \\
\hline Flat Plate Crush Strength, N/cm (lb/mm) & $245(5.5)$ & $265(6.0)$ \\
\hline Attrition Index ${ }^{(1)}$ & 99 & 99 \\
\hline Compacted Bulk Density, $\mathrm{g} / \mathrm{cc}\left(\mathrm{lb} / \mathrm{ft}^{3}\right)^{(2)}$ & $0.81(50)$ & $0.81(50)$ \\
\hline
\end{tabular}

(1) - Wt\% retained on 20 mesh screen after tumbling $1 \mathrm{hr}$ at $40 \mathrm{rpm}$

(2) - Sock loading density is estimated at $90 \%$ of CBD and dense loading density is typically $105-110 \%$ of CBD.

Table 3.4 - Catalyst Basket Dimensions

\begin{tabular}{|c|c|}
\hline Reactor Volume (Gal) & 5.0 \\
\hline Volume Used (Gal) & 2.5 \\
\hline Estimated Liquid Level (in) & 9.1 \\
\hline Reactor Length (in) & 19.5 \\
\hline Reactor Inner Diameter (in) & 9.0 \\
\hline Stir Rod Length (in) & 17.75 \\
\hline Impeller Diameter (in) & 4.0 \\
\hline Catalyst bulk density (Ib/in ${ }^{3}$ ) & 0.029 \\
\hline Basket Length (in) & 8.0 \\
\hline Basket Outer Diameter (in) & 6.5 \\
\hline Basket Inner Diameter (in) & 5.4 \\
\hline Basket Volume (in ${ }^{3}$ ) & 82.5 \\
\hline Amt of Catalyst (Ib) & 2.39 \\
\hline Basket Thickness (in) & 0.6 \\
\hline Free Space from basket to & 1.25 \\
\hline reactor wall (in) & \\
\hline
\end{tabular}




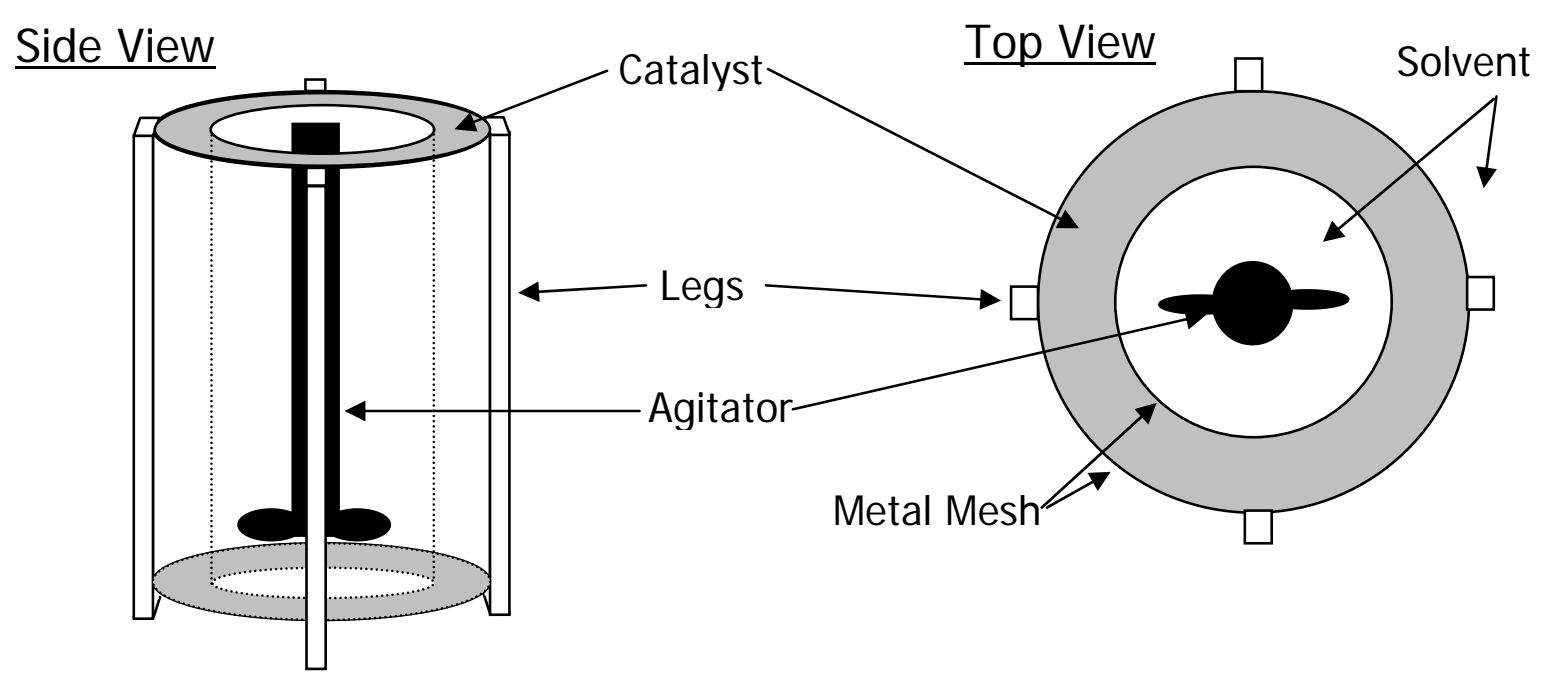

Figure 3.3 - Catalyst Basket Design

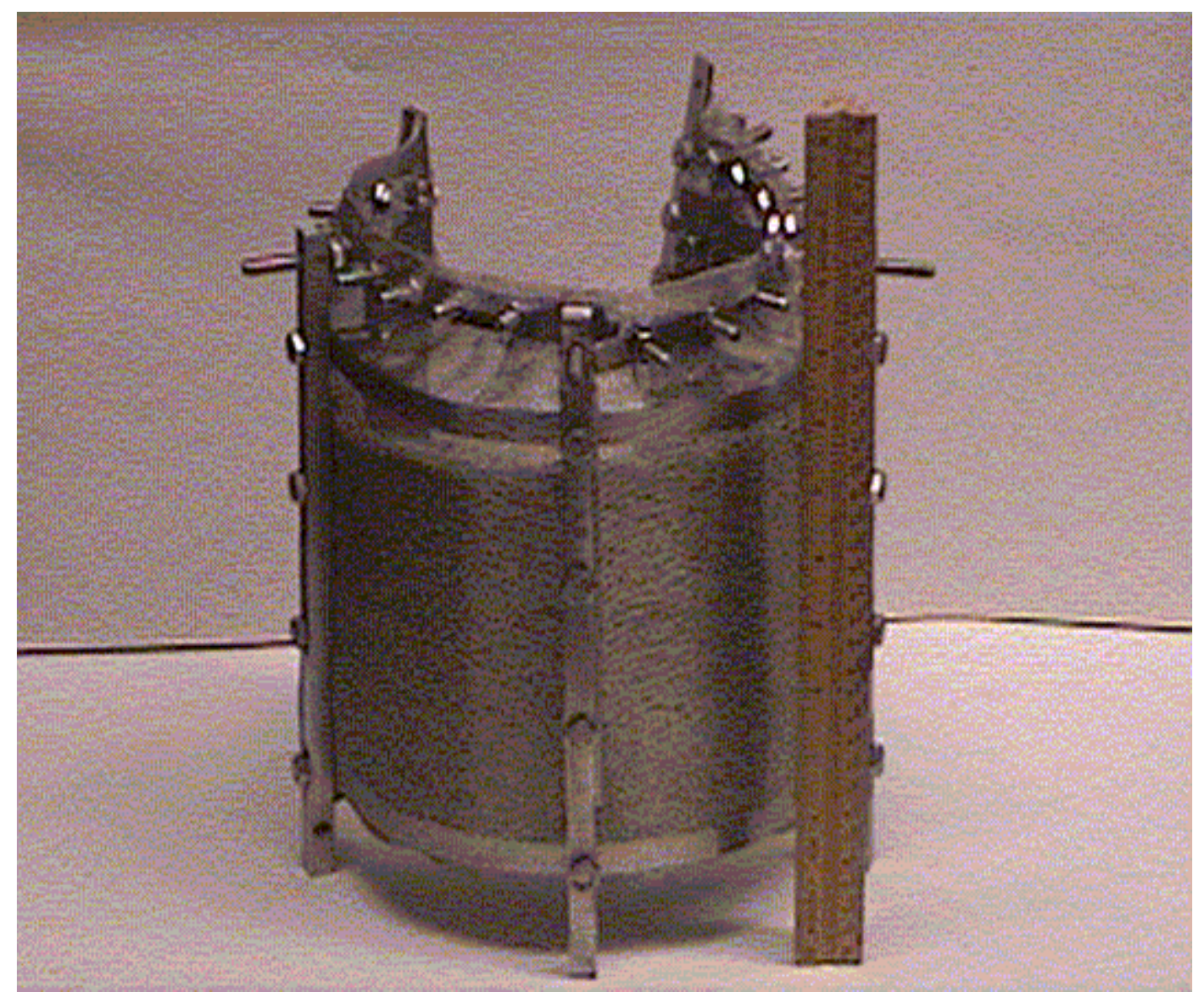

Figure 3.4 - Completed and Loaded Catalyst Basket 


\subsubsection{Catalyst Sulfiding}

The catalyst, as purchased, is a metal oxide combination. The most active form of these metals for hydrogenation is the sulfides. Therefore, a sulfiding step was necessary before any solvent treatment can begin. This sulfiding step was done according to the guidelines provided by Criterion. The sulfiding reactions occur at a temperature around $210^{\circ} \mathrm{C}$. Using the catalyst composition (see Table 3.3), it was calculated that $250 \mathrm{~mL}$ of dimethyl sulfoxide (DMSO - chemical formula $\mathrm{C}_{2} \mathrm{H}_{6} \mathrm{SO}$ ) would be sufficient to sulfide 2.5 pounds of catalyst (amount of catalyst used was rounded up to nearest half-integer). Once the catalyst had been weighed on the Mettler-Toledo PR 5002 scale, the catalyst was loaded into the basket from the top. This was done in a chemical fume hood, to prevent inhalation of any catalyst dust. The top of the basket was sealed shut, and manually shaken to ensure that the screen would prevent fines from escaping. The basket was then lowered into the 5-gallon reactor and the reactor was sealed shut using an electric impact wrench. Then each of the reactor bolts was tightened using a large torque wrench to $50 \mathrm{lbs}$, then $100 \mathrm{lbs}$, and finally $200 \mathrm{lbs}$. The DMSO was then loaded into the reactor through the top port, which was then sealed. The reactor heater was set to approximately $210^{\circ} \mathrm{C}$ and the impeller was turned on to $1000 \mathrm{RPM}$. Once the reactor temperature reached the desired set point, the timer was started and the reactor was held at temperature for one hour. The final pressure of the reactor at the end of the hour was 240 psig. The reactor heater was turned off, and the stirrer left on until the reactor cooled down. The reactor was vented while still warm, to prevent any un-reacted DMSO from condensing in the reactor. The reactor, while still warm, was pressurized up to 500 psig with cold nitrogen gas, and then vented. This process was repeated twice, and during the second venting process, the presence of a sulfur smell had decreased enough that it was 
felt it no longer necessary to continue the venting procedure. The reactor was kept sealed and unexposed to the atmosphere until needed.

\subsection{Solvent Hydrogenation}

Four solvents were utilized in this study to determine the effectiveness of solvent hydrogenation on the coal digestion. The 5-gallon reactor was loaded with Kopper's carbon black base to flush out any residual DMSO, emptied and then filled for the initial run with the CBB. The volume of solvent, as noted in table 3.4, was 2.5 gallons, or approximately $588 \mathrm{in}^{3}$. This volume would fill the reactor up to about 9.1 inches from the bottom of the reactor. Assuming the specific gravity of the warmed CBB was approximately 1.1 , the mass of CBB to be charged to the reactor was $27.5 \mathrm{lb}$ or $12.49 \mathrm{~kg}$. The specific hydrogenation conditions for each solvent are given in Table 3.5. Once the reactor was loaded with the solvent, the reactor was purged twice and pressurized with hydrogen gas up to the desired level (in the first run, this was 500 psig $\mathrm{H}_{2}$ cold). The reactor furnace was then heated to the reaction temperature while the impeller, controlled by the Reliance Electric SP500 VS Drive, rotated at $1000 \mathrm{rpm}$. The time, furnace temperature, reactor temperature, and reactor pressure were monitored during the entire process. Once the reactor reached the desired temperature, the reaction was allowed to proceed for one hour. At this time, the furnace was turned off, and the reactor was allowed to cool slowly. This typically took an overnight period, and the reactor temperature was at about ambient conditions when noted the following morning. The change in the cold pressure from before the reaction to after the reactor had cooled completely was recorded, as this is most likely the amount of hydrogen gas consumed. 
The reactor was then vented, and the furnace and impeller were then turned on again to warm the contents to allow for thorough draining. The contents were drained into 1gallon tin cans and the mass of product was noted. For this work, there were three "levels" of hydrogenation used on the solvents. The details of these are specified in the Table 3.5. In the instances when a different solvent was to be hydrogenated, that new solvent would be added to the reactor, stirred around while warm, and then drained without any reaction. This flushing out process was done to attempt to remove any residual solvent from the previous runs. For all solvents used in this research, simulated distillation and FTIR were performed on both the raw and hydrogenated solvents. This was done to analyze what differences existed between the raw and processed solvents.

Table 3.5 - Solvent Hydrogenation Reaction Conditions

\begin{tabular}{|c|c|c|c|c|}
\hline $\begin{array}{c}\text { Run \# I Hydrogenation } \\
\text { Level }\end{array}$ & Solvent & $\begin{array}{c}\text { Temperature } \\
\left({ }^{\circ} \mathbf{C}\right)\end{array}$ & $\begin{array}{c}\text { Pressure } \\
\text { (psig cold) }\end{array}$ & $\begin{array}{c}\text { Time } \\
\text { (hr) }\end{array}$ \\
\hline 1 / Level 1 & CBB & 275 & 500 & 1 \\
2 I Level 2 & CBB & 350 & 500 & 1 \\
3 / Level 3 & CBB & 375 & 750 & 1 \\
4 / Level 3 & SO & 375 & 750 & 1 \\
5 / Level 3 & MO & 375 & 750 & 1 \\
\hline
\end{tabular}

\subsection{Parametric Studies in Mini-Reactors}

The digestion ability of four base solvents and their hydrogenated variants was tested. Each solvent was either a coal processing or petroleum by-product. While this has been done with some success in the past by others, this investigation also looked into the viability of leaving the solvent in the final product, or co-processing the coal and the 
solvent. The coal digestions were performed in $30 \mathrm{~cm}^{3}$ tube bomb microreactors. Each tube bomb was fitted with a pressure gauge and valve to allow venting and pressurizing of the bomb. For all experiments, a solvent weight to coal weight ratio of 8:3 was chosen. The temperatures used in this study were $350^{\circ} \mathrm{C}, 400^{\circ} \mathrm{C}$ and $450^{\circ} \mathrm{C}$. Half of the runs were purged with nitrogen and half of them were pressurized up to 500psig. The products of the reactions were then extracted using THF as the solvent in order to calculate the coal conversion for each run. Each run was done in duplicate, to help validate the results. After the parametric experiments in the mini-reactors were complete, scale-up 1-gallon reactions were conducted using the best three solvents.

\subsubsection{Fluidized Sand Bath Preparation}

To heat the reactors for the hydrogenation runs, a Techne SBL-2 fluidized sand bath was used. The sand bath was filled to about $75 \%$ full with an aluminum oxide powder (app. 100 mesh). The temperature of this sand bath was maintained by the Techne TC-8D temperature controller, and the sand was fluidized by house air. The air flow was adjusted so there was a slight bubbling in the sand bath. This was low enough to prevent sand from escaping the overflow tray and high enough to ensure even heat distribution. To preheat the sand bath, the temperature was set above the desired reaction temperature (from $25^{\circ}$ to $50^{\circ}$ above reaction temperature, depending on the particular reaction temperature). The sand bath temperature was set to the desired temperature immediately prior to immersion of the reactors. 


\subsubsection{Tube Bomb Mini-Reactor Preparation}

Each tube bomb micro reactor would be cleaned out thoroughly before each use. As stated above, each run was done in duplicate. Each reactor's interior would be scoured out with a cylindrical wire brush and steel wool. The reactor end caps would also be cleaned with steel wool. Air was then blown in each cap and reactor to clean them out. Air was also blown down the reactor stems to remove any plugs or solid material left over from the previous use.

After cleaning, the reactors were capped on one end (typically the pressure gauge side). After placing the reactor in a vice, a small amount of copper anti-seize lubricant was applied to the threads on the reactor, and the Swagelock end cap was screwed on the end until hand-tight, and then tightened down with a wrench an additional quarter to half turn (see Figure 3.5). 


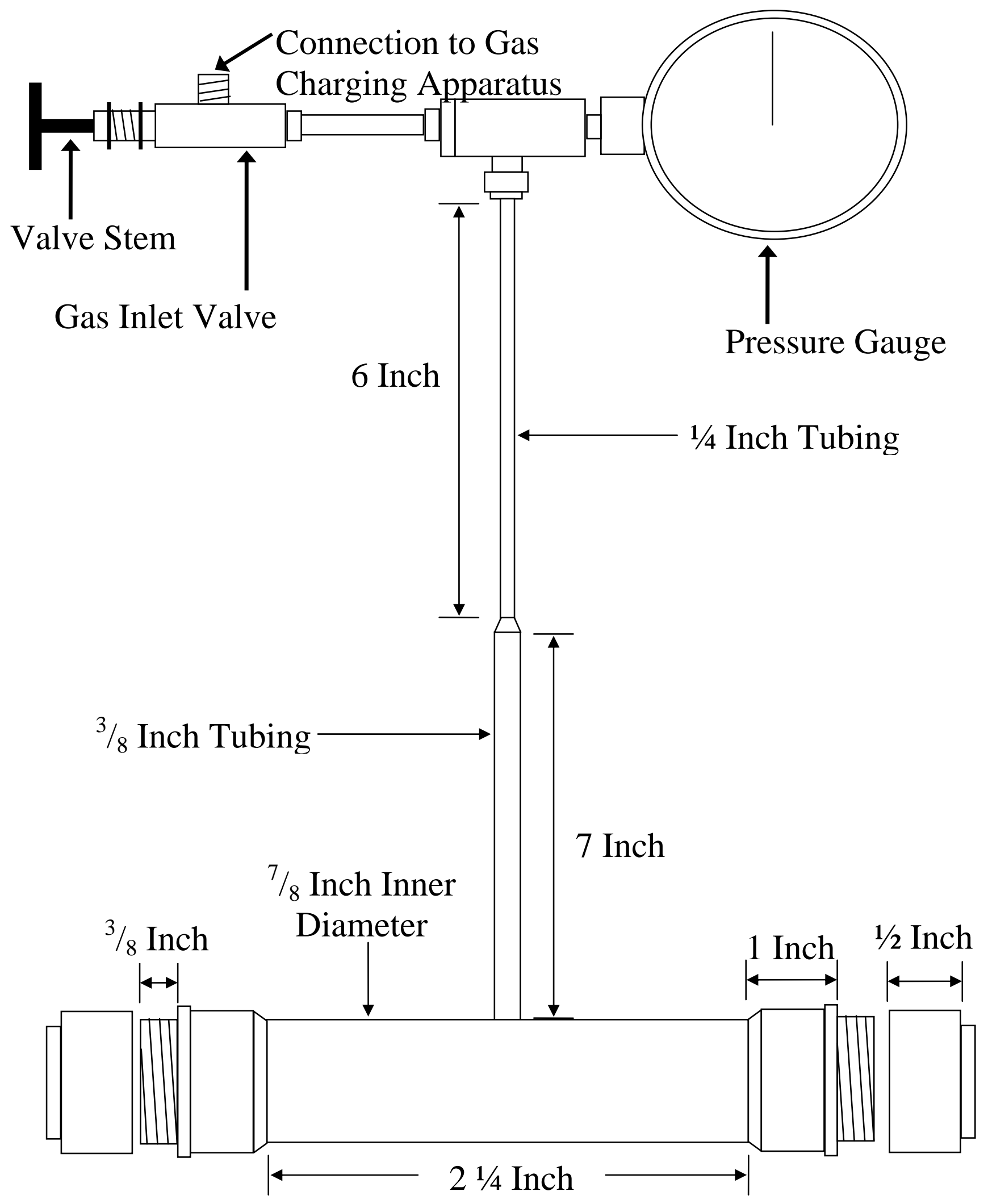

Figure 3.5 - Diagram of the Tube Bomb Mini-reactor 


\subsubsection{Tube Bomb Mini-Reactor Charging}

Each solvent was slightly different in texture and consistency. Carbon black base (CBB) was a paste at room temperature, while the other three were each thick, viscous liquids with varying amounts of solids. The CBB, AO and SO would each be heated in a oven (set at about $100^{\circ} \mathrm{C}$ ) for at least an hour, while being occasionally stirred, before a sample was obtained. While the MO was a viscous liquid, the viscosity was low enough to pour the material at room temperature.

The tube bomb would be placed into a small vice, which had been tared on the Mettler-Toledo PR 5002 scale. The empty reactor weight was noted. A sample of the solvent for this run was then placed into a small beaker. The beaker was then tared on a Denver Instruments Model A-200DS scale, so the amount of solvent could be determined by difference, since these solvents were all viscous fluids. The target weight for each run was $8 \mathrm{~g}$ total solvent. After the solvent had been added to the reactor, the coal (app. 3g) was then weighed in a small plastic tray and added to the reactor. After the solvent and coal had been loaded into the tube bombs, six (6) 316 stainless steel ball bearings were weighed and loaded to serve as agitators. The filled weight of the tube bomb was also noted. The same procedure used to place the first end cap on the reactor was then repeated to cap the other end of the reactor.

\subsubsection{Tube Bomb Mini-Reactor Pressurization}

Each tube bomb, once loaded and sealed, was pressurized with nitrogen gas, and tested for leaks. If there were any leaks, the caps were turned an additional quarter turn, or replaced if this did not work. If no leaks were detected, the tube bomb was then 
vented. This was done by opening the gas charging valve slowly, to prevent any fines from escaping the reactor or solvent from shooting up into the stem and causing a plug. The reactor was pressurized and purged two or three times with nitrogen. For each set of reaction conditions (solvent used, reaction temperature) there was a pressurized pair of reactors (nitrogen pressure of 500 psig) and an un-pressurized pair of reactors (purged with nitrogen but vented to atmosphere) to determine the effects of pressure on the coal digestion.

\subsubsection{Reaction Procedure}

After the reactors had been loaded, sealed and nitrogen purged/pressurized, they were mounted onto the shaking mechanism over the sand bath. The sand bath, which had been preheated, was set to the desired reaction temperature, and then raised up via a wench and pulley system to a level just below the reactors. The shaker was turned on at this point to a very low setting, and then the sand bath was raised the rest of the way, until the reactor bodies were completely submerged into the sand. Two formed sheets of metal were then placed on top of the sand bath, with a gap left open for the reactor stems, to prevent the sand from escaping the bath. Next, the shaker controller, a Dayton Industries DC Speed Controller, was turned up to a level of approximately 40-45. This corresponded to a shaking speed of about 500 rpm, with a stroke length of about 2 inches.

At this point, the time was noted, and the reaction was allowed to proceed for a total time of one (1) hour. The sand bath temperature and the reactor pressures were noted every 10-15 minutes. At about 58 minutes into the reaction, the shaker mechanism was turned off, and the sand bath lowered. The excess sand was shaken off of the 
reactors, and then the reactors were removed from the shaker mechanism and immediately placed into a cold water bath to quench the reaction. This was done at 58 minutes into the reaction to allow for the time to lower the sand bath, loosen the bolt keeping the reactors mounted, and then remove the reactors and take them to the water bath.

\subsubsection{Product Collection}

To determine the effectiveness of a particular solvent/solvent combination in the conversion of coal, the products of the coal digestion were subsequently processed by THF Extraction. For each reactor, a marked 250-mL glass round flask was weighed and placed on a ring stand. Two Teflon boiling chips were also weighed and added to the flasks. A glass funnel was placed on the flask, and in the funnel was placed a weighed Whatman Glass MicroFibre Thimble (19mm ID, $90 \mathrm{~mm}$ length). Another glass funnel was placed on a ring clasp above the thimble, with the tip of the funnel just down into the thimble (see Figure 3.6).

The reactor was then weighed, after air blowing all the water and sand off of the reactor body, to get an after-reaction weight for use in determining the mass of any gas produced in the reactor. Once this weight was obtained, the reactor was set into a fume hood and the gas charging valve was slowly opened to vent the reactor. Most of the unpressurized reactors had a negligible amount of gas produced during the reaction, and there was very little pressure to vent. Care was taken during venting to make certain that no liquid product was accidentally vented along with the gas. On certain samples where the product was likely to be a less viscous mixture, the reactors were placed in a 
container full of dry ice and allowed to cool, and then vented. Once the reactors had been vented, they were weighed again.

The reactor was next place back in a vice, and the end cap on the valve stem side was loosened. The threads were wiped clean using tissue paper, to remove the copper lubricant. The reactor was then suspended over the funnel-thimble-funnel-flask setup. A heat gun was typically used to warm up the products enough to allow them to flow out of the reactor and down into the funnel. After the majority of the material had emptied out of the reactor, the reactor was then turned over and allowed to cool a bit, and then THF was poured into the reactor. A metal spatula was used to scrape the sides of the reactor, and then this was poured out into the funnel. This process was repeated, usually four or five more times, until the liquid poured out was close to being clear. Care had to be taken to keep the liquid level in the thimble from getting too high and causing the thimble to overflow. THF was also sprayed down the stem of the reactor from the gas charging valve to clear out any product that had moved up into the stem. In the event that there was a clog, the top part of the reactor apparatus (valve, pressure gauge, etc.) would be removed, and the clog would be removed by using a length of copper wire.

Any product in the end cap was scraped out with a metal spatula, and then the caps were washed with THF. Finally, the other end cap was removed from the reactor, the threads were wiped clean of copper lubricant, any product in this cap was scraped and then washed out, and then THF was sprayed into the reactor to clear out any remaining particulates. Once this was done, the funnel was washed with THF (and scraped if necessary), until all the product was in the thimble. During this process, it was occasionally noticed that a gooey material would collect around the base of the thimble in 
the funnel. Since it had made it through the thimble, it was decided to include this material in the soluble portion, and any of this present would be washed into the flask.

For any reactions that resulted in a more solid or chunky material in the product (mostly the slurry oil runs), the material would be chipped out of the reactor with metal spatulas, and then the washing process would be used to clean out the reactor. Great care had to be taken to keep any product from falling out of the reactor and not into the funnel. Once this had been done, all of the product would be in either the thimble or flowing through the thimble with the THF and into the flask.

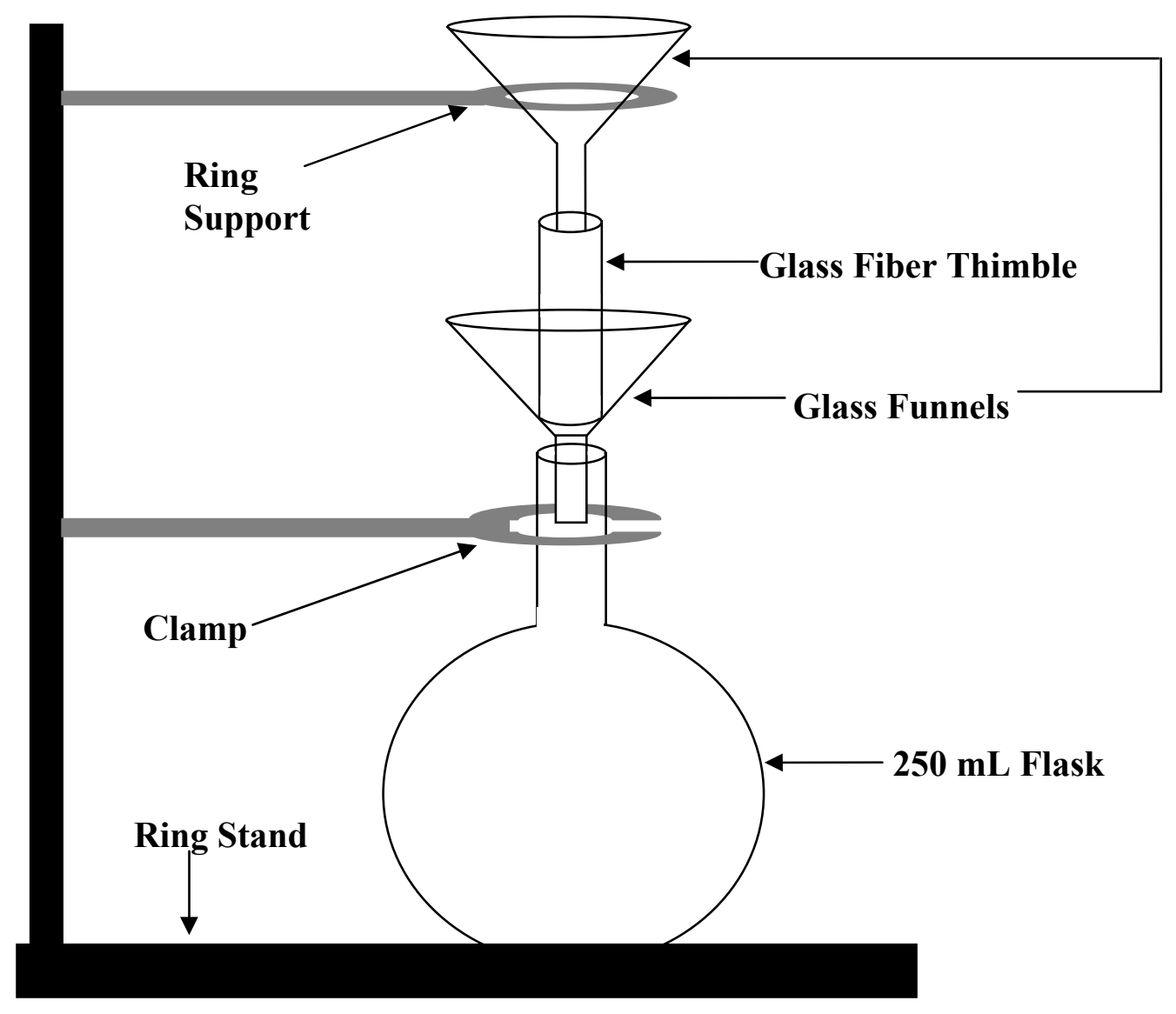

Figure 3.6 - Product Removal Thimble / Flask Apparatus 


\subsubsection{THF Extraction}

Once all of the products had been removed from the reactors, and the level of liquid in the thimble had receded down below the bottom of the funnel, the top funnel was removed. The thimble was carefully lifted from the bottom funnel and placed down into a glass Soxhlet extractor. The bottom funnel was then washed (and scraped if necessary), and the flask was filled with additional THF until it was about 2/3 to $3 / 4$ full. The flask was then fitted to the bottom of the Soxhlet, and this was then placed onto a heating mantle (see Figure 3.7). The top of the Soxhlet was fitted with a water cooled condenser. The cooling water was then turned on, and the heating mantle temperature was adjusted to get above the boiling point of THF (approximately $75^{\circ} \mathrm{C}$ ). This was done using a variable autotransformer, also called a variac, set at about 60-62 on the dial. Once the contents of the flask began to boil, this was left to boil and reflux overnight, until the liquid in the Soxhlet had become mostly clear, signifying that all the soluble material in the thimble had been dissolved and moved into the flask.

The variac was then turned off, and the cooling water left on, to allow the mixture to cool to room temperature. The liquid in the Soxhlet was emptied into the flask, and the thimble was allowed to drain into the soxhlet. Once the Soxhlet was mostly or all dried, the thimble was removed with tweezers and placed into a marked beaker to keep track of what run it was from. The Soxhlet was washed with THF and then emptied into the flask, and the flask was placed on a Buchler Instruments Rotary Evaporator using an oil bath set to $90^{\circ} \mathrm{C}$ to remove THF. After most of the THF had been removed from the flask, the temperature was increased to $110^{\circ} \mathrm{C}$ and left to run for a few minutes, and then a vacuum was applied. This was done until the boiling in the flask stopped. Care was taken to make certain that only the THF, not any product, was removed during the 
application of vacuum. After the flask had been allowed to cool, it was wiped clean of the oil and then taken to the Denver Instruments model A-200DS scale to be weighed. This mass was the THF soluble portion of the reaction products. The beaker containing the thimble was placed into a vacuum oven set to about $80-90^{\circ} \mathrm{C}$ and under a vacuum of 25-30 mm HG. A nitrogen purge was used to keep air out of the vacuum oven, and this was usually allowed to run overnight. The heat to the vacuum oven would be cut off, and it was allowed to cool, usually for a few hours. After the oven had cooled, the vacuum was broken, and the thimbles were weighed on the Denver Instruments model A-200DS scale. After subtracting the clean, dry thimble weight and the weight of the ball bearings, this was the THF insoluble portion of the reaction products. For the purposes of calculating a conversion percentage, it was assumed that all of the THF insoluble portion would have come from the coal and not from the solvent. The coal conversion was calculated using Equation 3.1.

\% Coal Conversion $($ daf $)=\frac{[(\text { Mass Dry Coal })-(\text { Mass THF Insolubles })] \text { X100 }}{\text { Mass Coal }(\text { daf })}$ Eqn 3.1

As an explanation on solvent choice, THF was used in this process, rather than NMP (N-methyl pyrrolidone), which has been the solvent of choice traditionally at West Virginia University. NMP has a much higher boiling point (app. $202^{\circ} \mathrm{C}$ ) which is in the range of many light hydrocarbon materials which would likely be present in the reaction products. During the rotary-evaporation step, this would likely lead to a loss of the soluble product while the NMP was removed. This was undesirable, and THF, having a 
much lower boiling point, was thought much better in determining coal conversion percentages.

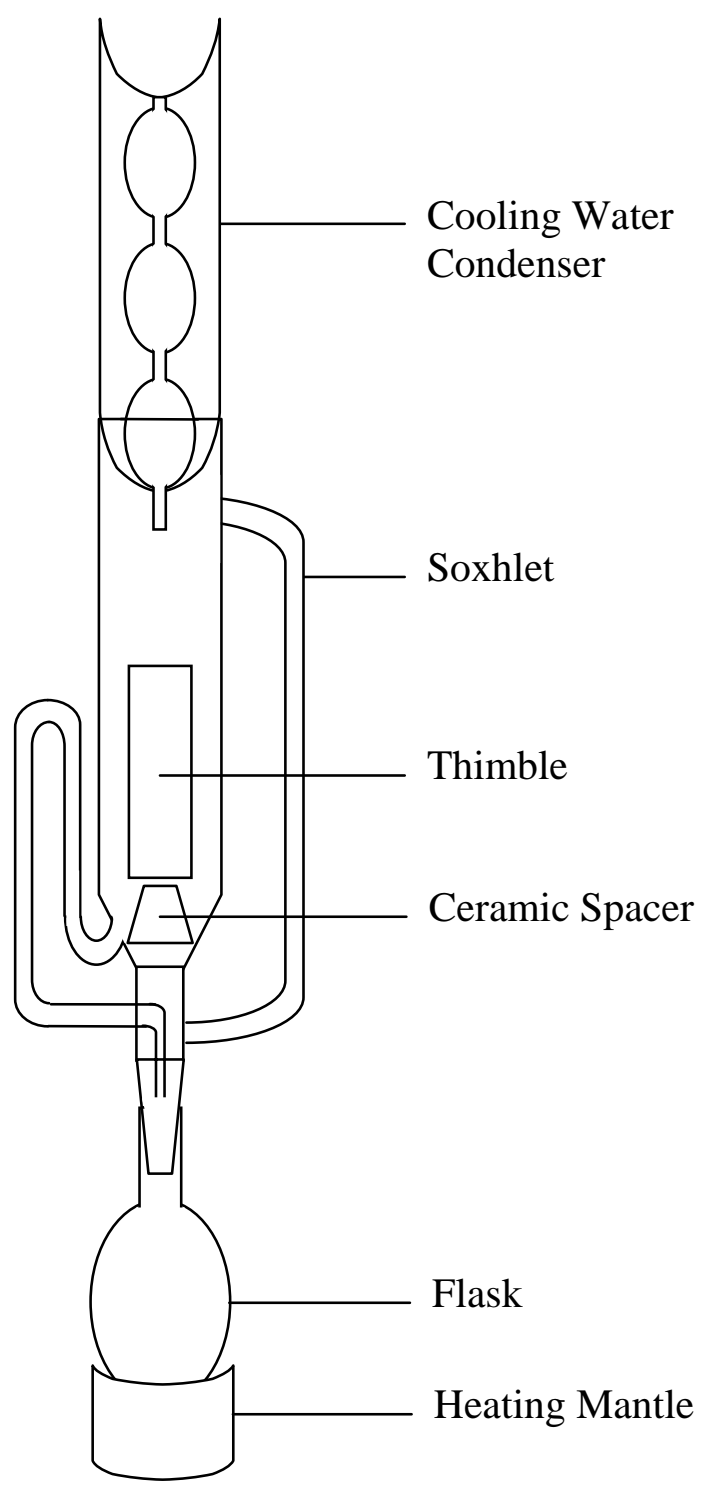

Figure 3.7 - Soxhlet Condenser Apparatus 


\subsection{One Gallon Reactor Scale Up Studies}

The next step in this study was to produce a more sizeable quantity of pitch precursor using the best three solvents, based on the results of the tube bomb digestions. This coke precursor would then be separated from the unconverted coal and thermally treated using an air blowing technique. The same coal and solvents used in the tube bomb reactions would be used in this scale up. The ratio used in the tube bomb experiments was kept for this scale up operation, along with the time, temperature and the pressure. For this scale up, based on the results from the tube bomb reactions, the solvents used were HCBB-L3, HSO-L3, and the 50/50 mixture of these two, as well as the HMO-L3.

\subsubsection{One Gallon Reactor Preparation}

The 1-gallon reactor (see Table 3.6), made by Autoclave Engineers, was first pressure tested to ensure it would hold the proper pressure. After several leaks were detected, the reactor was shipped back to the Autoclave Engineers to be retooled. After it was returned, pressure testing was again conducted, this time successfully. The reactor, having been retooled, was fairly clean and only required some wiping down with a solvent to remove any residual oil on the reactor. The lid was fitted with the required plugs, thermowell and stirring mechanism. 
Table 3.6 - One Gallon Reactor Dimensions

\begin{tabular}{|l|c|}
\hline Reactor Size (Gal) & 1 \\
\hline Reactor L (in) & 12 \\
\hline Reactor ID (in) & 5 \\
\hline Stir Rod L (in) & 11 \\
\hline Impeller D (in) & 2 \\
\hline
\end{tabular}

\subsubsection{One Gallon Reactor Charging}

Since the reactor was significantly larger, the amounts used would be increased to approximately 1.5L of solvent (measured in graduated beakers) and $600 \mathrm{~kg}$ of the coal. The solvent and coal masses were recorded from the Mettler-Toledo PR 5002 scale. Again, the solvent was loaded into the reactor first, to prevent any sort of caking effect on the coal at the bottom of the reactor. After the reactor was fully loaded with solvent and coal, the reactor lid was placed onto the reactor, and the lid bolts were tightened with the electric impact wrench. Next, the bolts were each tightened with a large torque wrench to $50 \mathrm{lbs}, 100 \mathrm{lbs}$, and finally $150 \mathrm{lbs}$. The reactor was purged with nitrogen gas, but left at 0 psig for the reaction.

\subsubsection{One Gallon Reactor Coal Digestion}

The temperature of the reaction was maintained by using the Autoclave Engineers Modular Control Series Process Controller. The impeller was turned on to $1000 \mathrm{rpm}$ and the reactor heater turned up to the desired reaction temperature. The first of the three reactions (HCBBL3) was run at the same conditions $\left(400^{\circ} \mathrm{C}\right.$ and 0 psig for one hour) as 
the tube bomb reactions. The subsequent reactions were run at a higher temperature of $425^{\circ} \mathrm{C}$, for reasons to be explained in the results, Section 4. The reactor pressure and temperature were monitored during the hour. At the end of the reaction time, the heater was turned off and the impeller was left on while the reactor cooled down.

\subsubsection{One Gallon Reactor Products Collection}

Once the reactor had cooled down to an acceptable temperature, the bolts were loosened on the lid, and then the lid was carefully taken off. This was all done while the reactor was still in the fume hood, since there would likely be noxious fumes as the products were still at a slightly elevated temperature (usually at least $100^{\circ} \mathrm{C}$ ). The products from these reactions were recovered using a vacuum line and an Erlenmeyer flask with a sidearm. Once the reactor had been emptied into the flask, the contents of the flask were reheated in an oven set to $\sim 100^{\circ} \mathrm{C}$, and then the product was transferred to plastic, pre-weighed centrifuge bottles. The bottles were weighed on the Mettler scale and then placed into the Thermo Electron Corporation PR700M Centrifuge and ran for 30 minutes at $4000 \mathrm{rpm}$ and $39^{\circ} \mathrm{C}$, which was the maximum temperature allowable for operation of the centrifuge. After centrifugation, the liquid product was decanted out of the centrifuge bottles into a glass, pre-weighed bottle and then the product mass was determined. This was the effective converted portion of the coal, and the residue from the centrifugation was then put through a simple warm THF filtration, to remove any entrained product still left in the residue. This was done in an effort to close the mass balance and to determine coal conversion. The THF insoluble portion left after filtration was dried in a vacuum oven, and this true unconverted coal along with any ash material. 
The THF was stripped off of the soluble portion using the rotary evaporator, and this was also vacuum dried overnight. The conversion was calculated according to Equation 3.3. These products were tested using proximate analysis (to determine ash and fixed carbon), simulated distillation, FTIR and elemental analysis.

\subsection{Air Blowing Studies}

The results of the scaled up digestions each underwent additional treatment by air blowing to convert the material into a coker feed. Air blowing was chosen as the treatment due to the effectiveness that was demonstrated in a recent study ${ }^{(4)}$.

\subsubsection{Air Blowing Reactor Preparation}

The air blowing reactions were done in a one-liter autoclave reactor. This reactor was scoured out with a wire brush and steel wool before the first use and after each use. The lid was fitted with an air tube, which, based on previous experience, was bent and then tied, with copper wire, to the thermowell. This was done to prevent the long air inlet tube from getting tangled up in the agitator as the mixture became more viscous during treatment. The tube was long enough to ensure that the air was blown into the material and not on top of it. This was also learned from experience. The lid was also fitted with the agitator. The reactor body was fitted with a distillate tube, wrapped in heating tape, so any volatiles evolved from the process could be condensed and collected. 


\subsubsection{Air Blowing Reactor Loading}

Once the reactor had been prepared, the coal digest was weighed and loaded into the reactor. A metal container was weighed empty and used as the distillate collection container. The weight used in this study was kept at about 300 grams, since previous experience in air blowing tests had shown that any more precursor material charged into the 1-liter reactor would result in excessive turbulent mixing and product being blown up into the vent line, which led to clogging. The furnace was then placed around the reactor body, and the temperature controller was turned on to begin to warm up the pitch precursor. The air flow was turned on and the reactor lid was then attached to the reactor. Since this was a process run at atmospheric pressure, the reactor lid bolts were only tightened with the electric impact wrench. The agitator was then turned on to $750 \mathrm{rpm}$, and the air flow was adjusted to approximately $1.2 \mathrm{~L} / \mathrm{min}$ on the Riteflow $150 \mathrm{~mm}$ flowmeter, and the heating tape on the distillate tube was turned on. The reactor setup used is shown in Figure 3.8. 


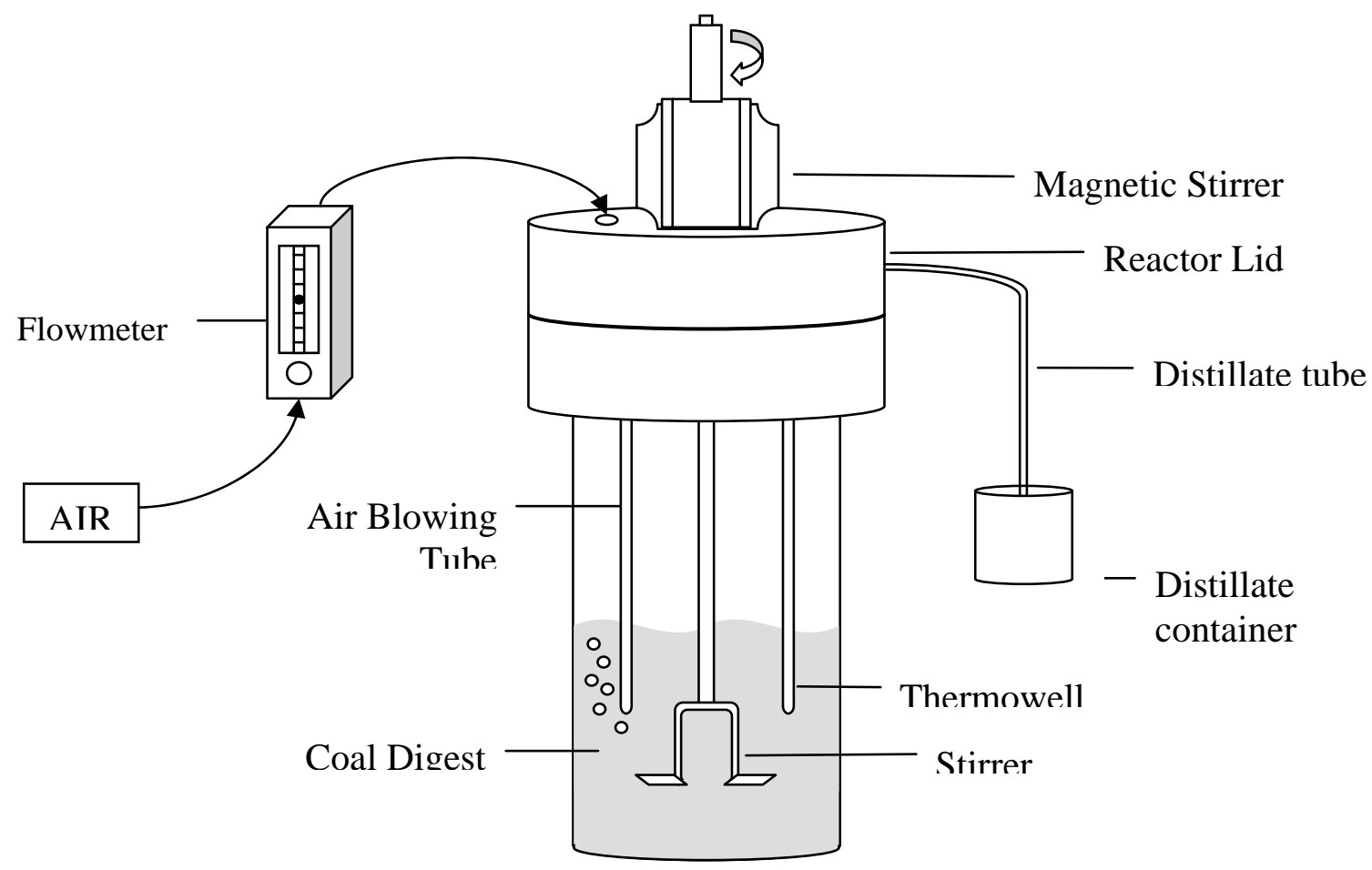

Figure 3.8 - Air Blowing Reactor Setup

\subsubsection{Air Blowing Reaction}

Once the reactor had reached the desired temperature, the timer was started. Air flow was monitored to make certain no clogs developed. The container placed under the distillate tube was typically covered with some fiberglass insulation, to assist in preventing any cooling which would cause clogs. The reaction conditions for the samples were kept identical, for comparative reasons. The reactor temperature was quickly brought up to $250^{\circ} \mathrm{C}$, and was held here for five (5) hours.

\subsubsection{Air Blowing Reactor Product Recovery}

Once the desired reaction time had elapsed, the heater and the agitator were both turned off. The distillate tube was removed, and the reactor lid bolts were loosened, all 
while the air flow continued. Once the reactor lid was unbolted, the lid was lifted off of the reactor, taking care of the agitator and the thermowell/air flow tube. The lid assembly was laid into a large metal pan and set aside. The reactor was then lifted off of the stand, and quickly placed into a cold water bath in a nearby sink. This was to quench quickly the reactor. A watch glass was placed on top of the uncapped reactor to prevent vapors from escaping into the air, and the reactor was allowed to cool off.

Once the reactor had cooled, the now solid material in the reactor was chipped out with a hammer and a spike. Dry ice was used in the cases where the product was gummy or still slightly ductile, to allow for easier chipping out of the reactor. Any product stuck on the stirrer was scrapped off into a pan used to collect the digest product from the reactor. The pan had been weighed empty, and was used to weigh the product and calculate the yield after air blowing. The product was then transferred into a plastic container which was stored in a cold room. The distillate container, which had also been pre-weighed, was also weighed and the amount of the distillate collected was then calculated.

\subsection{Analytical Testing}

The properties of the coal, solvents, coal digest liquids and air-blown digests obtained during this study were evaluated so as to assess the changes occurring during the processing, as well as to ascertain the viability of the final air-blown digests to be used as coke precursors. The evaluation tests included the softening point, ash content, coke yield, elemental analysis, density, viscosity, simulated distillation, Fourier-Transform infrared spectroscopy (FTIR), and toluene solubility. 


\subsubsection{Softening Point}

The softening point of a pitch material is required to characterize the air-blown digest. It is a measure of the temperature at which point the material will flow a particular distance. This property helps define the rheological consistency of the airblown digest material.

The softening point is determined according to ASTM 3104 procedure. In the laboratory, the test was done, in duplicate, using the Mettler FP83 HT drop cell, controlled by the Mettler FP80HT central processor. A sample of the air-blown digest was melted, carefully to prevent any excess oxidation or smoking, in a sample cup on a hot plate. This sample cup has an opening in the bottom of it, to allow the sample to flow out of it in the Mettler Drop Cell. Once the sample cup was properly loaded, a small lead ball was placed on top of the sample, and once it had cooled, the sample cup was attached to the rest of the drop container. The sample was then placed into the Drop Cell, and the ASTM procedure mentioned above was followed to determine the softening point.

\subsubsection{Ash Test}

This test is done to measure the ability of the solvent to remove or reduce the ash content in the original coal from the final digest product. This test was done according to ASTM D2415-98, again, in duplicate. Ceramic crucibles were heated to a red hot state, to drive off any moisture, and then placed into a desiccator to cool. Once cool, they were weighed, and the appropriate amount of the sample was placed into the crucible. The crucible was placed into the programmable oven and the lids were placed onto the crucible, left slightly open to allow for air flow into the sample for proper ashing. The 
temperature was ramped up in the oven at $5^{\circ} \mathrm{C} / \mathrm{min}$ up to $400^{\circ} \mathrm{C}$ and then at $3^{\circ} \mathrm{C} / \mathrm{min}$ up to $750^{\circ} \mathrm{C}$. This temperature was held for 180 minutes to ensure complete combustion, and then the oven temperature was lowered to room temperature. The crucible was carefully weighed, and the difference in weights (pre-heat less post-heat) was divided by the original sample weight to calculate the percent ash in the sample.

\subsubsection{Coke Test}

These are measures of the non-volatile material present in a pitch material. There are two different tests used regularly in studies conducted here at West Virginia University

\subsubsection{Conradson Carbon Test}

This test was done according to ASTM D189. Just as in the ash test, the crucibles to be used in this test were first heated to a red-hot state, and then cooled in a desiccator to remove any moisture. Then the crucible was weighed, and the sample was weighed (between 0.4 and $0.6 \mathrm{~g}$ ) and added to the crucible. This ceramic crucible was then placed into a metal crucible, which was then covered by a lid, and then placed into a larger metal crucible, with a small amount of Coke Breeze on the bottom, and then covered itself by a lid. Coke Breeze was used to scavenge the oxygen in the crucible during the process. Once the crucible was prepared, it was placed in a Meker-style burner. The crucible setup was heated at a "medium" level flame for 11.5 minutes, then at a "low" level flame for 13 minutes. This was the de-volatilization stage of the test. Lastly, the flame was turned up to a "high" level, and this was held for 7 more minutes. The crucible was then 
removed from the heat and placed aside to cool. The lid was kept on to prevent any oxygen from entering the system and causing any combustion to occur. After the crucible has cooled some, the ceramic crucible was removed from the setup and placed into a desiccator to cool completely. The crucible was then weighed, and the Conradson Coke value was calculated as the percentage of the mass remaining based on the original mass of the air-blown digest.

\subsubsection{WVU Coke Value}

This test was developed by the researchers at West Virginia University, and cokes a sample at a slower rate, which allows for better optical texture formation during the process than the more severe Conradson test. The sample crucible was heated red-hot, and then placed into the desiccator to cool. The crucible was then weighed, and the weighed sample (0.4-0.6 g) was added to it. Next, a small amount of coke breeze (an oxygen scavenger) was placed into a larger ceramic crucible, and the sample containing crucible (with lid) was placed into the larger crucible. Then this crucible was filled to the top (making sure to completely cover the lid of the sample crucible) with coke breeze, and a lid was placed on this crucible. This test was run in the programmable oven, and the heating rate was $5^{\circ} \mathrm{C} / \mathrm{min}$ up to $600^{\circ} \mathrm{C}$. This temperature was held for 120 minutes, and then the oven was cooled down to room temperature. Once cool, the smaller crucible was removed and weighed, and the WVU coke value was obtained via equation 3.2.

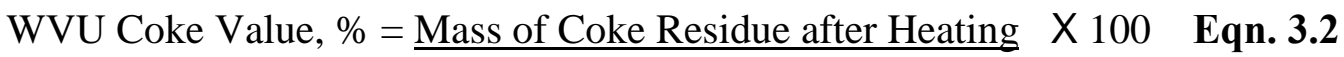
Mass of original sample
} 


\subsubsection{Elemental Analysis}

This was performed on all of the solvent samples, the coal and the coal digest liquids and air-blown coal digests. This test was done using the Thermoquest Flash EA 1112 equipped with two reactor columns. The first column gives the percentages of Carbon, Hydrogen, Nitrogen and Sulfur present in the sample. This is done by converting each of the elements present in the sample (only 2 to $3 \mathrm{mg}$ ) into their respective oxide ( $\mathrm{C}$ goes to $\mathrm{CO}_{2}, \mathrm{H}$ goes to $\mathrm{H}_{2} \mathrm{O}, \mathrm{N}$ goes to $\mathrm{NO}_{2}$, and $\mathrm{S}$ goes to $\mathrm{SO}_{2}$ ). These gases passed through a gas chromatographic column, where they were separated and analyzed. The second column gives the percent of the Oxygen in the sample, however, for the purposes of this study, the percent Oxygen was calculated by difference. These tests were done in quadruplicate. Vanadium pentoxide (usually about $3 \mathrm{mg}$ ) can be added to the sample containers to promote combustion, specifically with the air-blown samples.

\subsubsection{Density}

The density of the pitch was determined using the AccuPyc 1330 helium pycnometer according to ASTM 2320-98. From prior experience, air is often emulsified into the pitch samples taken from the air-blowing reactor. This can distort the density measurement, so to counteract this, samples of the pitch were annealed (at $100^{\circ} \mathrm{C}$ over the softening point, if the softening point was less than $200^{\circ} \mathrm{C}$, for 20 minutes, or for 30 minutes if the softening point is over $200^{\circ} \mathrm{C}$ ) in a crucible placed in an oven. This allowed the air trapped in the pitch to escape so an accurate density measurement could be taken. 


\subsubsection{Viscosity}

The viscosity of the coal liquid digests and the final air-blown digest products was determined using a Brookfield DV-III Rheometer, and the test was done according to ASTM D-5018-89. The sample chamber was filled with approximately 12 grams of pitch, and then placed into the rheometer, where it was then heated up to approximately $15-20^{\circ} \mathrm{C}$ above the softening point of the pitch. The test uses the Brookfield SC4-34 spindle, which delivers defined shear rates in order to determine shear stress. From these data, a dedicated computer system calculated the viscosity of the pitch at that particular temperature. Once this was done at the initial temperature, the temperature was raised $10^{\circ} \mathrm{C}$ and method repeated, until the pitch viscosity was less than $1,000 \mathrm{cP}$. The data obtained was viscosity versus spinning rpm versus temperature.

\subsubsection{Toluene Solubility}

This test was done to determine the amount of insoluble material in the final digest and air-blown digest products. The test was done essentially the same as the THF extractions that were performed on the tube bomb reactor products (Section 3.4.7); this time, however, the solvent was toluene. The same funnel-thimble-funnel-flask setup was used, and very similar procedures were followed. First, a beaker with about $125 \mathrm{~mL}$ of toluene was set on a stirring hot plate and a Teflon coated stirring rod was placed into the beaker. The stirrer was turned on, and the sample to be extracted was then weighed out. Approximately 3 grams of each sample was used, and once weighed out, it was added to the beaker of toluene, and the heat was turned on and adjusted so that the toluene would be heated to a point right around the boiling point, and a watch glass was placed on the 
top of the beaker to allow for refluxing of the toluene. The toluene refluxed for about 15 to 20 minutes, and then the beaker was removed from the hot plate. The stirring rod was removed from the beaker, and rinsed. Then the beaker was carefully emptied out of the beaker into the funnel-thimble-funnel-flask apparatus (Figure 3.6). After the beaker had been emptied and rinsed a couple of times, the thimble was placed into a Soxhlet refluxer and this was fitted onto the flask. This apparatus was then placed onto a heating mantle and fitted with a condenser. The toluene refluxed overnight, until no more pitch material leached out of the thimble. Once drained, the thimble was removed from the Soxhlet and dried in a vacuum oven, while the flask was heated in the rotary evaporator to remove the toluene, and then this too was dried overnight in a vacuum oven. The weights were recorded and the percent toluene insolubles and solubles were calculated.

\subsubsection{Simulated Distillation}

This was done using a Varian CP-3800 Gas Chromatograph, fitted with a special auto-sampler (model \# 8410) for liquid samples and special software, Star SD version 6.2, for simulated distillation. The samples were prepared in small $2 \mathrm{~mL}$ vials, and the solvent used was carbon disulfide $\left(\mathrm{CS}_{2}\right)$. A blank was prepared using $\mathrm{CS}_{2}$ only, and then a small amount of the sample to be tested (app. 2-3 mg) was placed into the vial, which was then filled with $\mathrm{CS}_{2}$ and shaken to mix thoroughly. This dilution should typically be a fraction of about $1 / 100$, which should be adequate for the analysis. Weights were not required, as the process is done on a volume basis. Once the samples were prepared, they were placed into the auto-sampler tray, along with a wash container of $\mathrm{CS}_{2}$, and the Sim-

Dist software was started. The software requires a method, a sample list, and prior 
preparation of the GC itself. For these runs, the Column and Injector temperatures ranged from $35^{\circ} \mathrm{C}$ to $425^{\circ} \mathrm{C}$ (this is the recommended maximum column temperature for the particular column used). Only one flame ionization detector (FID) was utilized, and the temperature was set to $380^{\circ} \mathrm{C}$. This requires both compressed air and hydrogen gas (at rates of $300 \mathrm{ml} / \mathrm{min}$ and $30 \mathrm{ml} / \mathrm{min}$, respectively). To prepare the method, a standard method's parameters were compiled, and the temperatures and other settings were modified as described above to fit the desired analysis. This method was saved and then activated, which started the heating/cooling of the oven, detector and injector. To prepare a sample table, a new sample table was opened, and the samples labeled according to their position in the sampler tray. At the beginning of the table, the $\mathrm{CS}_{2}$ sample was labeled a "Baseline", and the actual samples were marked for "Analysis". At the end of the table, the "Sleep Sim-Dist" method was set to activate, which caused the machine to go into its "Sleep" mode, which closed the gas valves and cooled off the ovens. Once the sample table was prepared, the "Begin" button was pressed, and the program took over. Each sample run lasted for approximately 40 minutes, but the cool-down in between the runs could take over an hour. After the data has been collected, the chromatograms were then analyzed by the StarSD software, and when compared with the appropriate baseline background $\left(\mathrm{CS}_{2}\right)$, boiling point distributions can be obtained. These data can then be analyzed via differentiation to determine if the peaks in the derivative correspond to the digestion efficiency, air-blown softening point or coke yield. 


\subsubsection{Fourier-Transform Infrared Spectroscopy (FTIR)}

Fourier-transform infrared spectroscopy (FTIR) was done to look at the aromaticity and functional group changes that occurred with the solvents, coal digest liquids and air-blown digests during the various processing performed. The test was done using a Nicolet 510P FT-IR Spectrometer, and was done with a KBr Diffuse Reflection method for the coal digests and the air-blown products and with the ATR (Attenuated Total Reflectance) method for the solvents.

The ATR method was used for liquids that cannot easily be made into solid pellets. A sample of the solvent was poured onto the $\mathrm{Zn}$-Se plate, which had been run clean as the background. This was then placed into the FTIR machine and the sample was taken.

To perform the FTIR on the digests, about $300 \mathrm{mg}$ of potassium bromide (KBr) were weighed and added to a metal sample capsule. Next, a small dab of coal digest (liquid) or air-blown pitch (solid) was added to the capsule. The capsule was then capped and a small piece of parafilm was wrapped around the cap to prevent any sample from escaping. The capsule was then placed in a Wig-L-Bug shaker mechanism and shaken for 2 minutes at $3800 \mathrm{rpm}$. After this was done, the parafilm was removed and the capsule was tapped on the counter firmly to keep the sample from remaining in the cap when it was removed. The cap was then removed and the contents were carefully poured into the sample holder tray. A small spatula was used to smooth out the sample level in the holder tray, and the holder tray was then placed into the FTIR machine. The tray was placed into the instrument carefully to make certain that the laser would hit the center of the pellet. The instrument was purged with dry air for approximately 15 minutes and then the analysis was run. 
Before any samples were analyzed in the FTIR, a background spectrum must be run. This was a KBr-only sample, made in the same manner as the pitch and coal digest samples. This must be done every day, as $\mathrm{KBr}$ will readily absorb any atmospheric moisture, to ensure that the correct background signal can be subtracted from the sample readings. Once the background was run, the samples could be run; as many as needed, provided the background is regularly redone.

\subsection{Experimental Error}

In order to determine the accuracy and consistency of the experimental procedures presented herein, a percent relative error has been calculated for the various quantitative characterization techniques. Unless otherwise indicated, all error in this report is given as a relative error. This value is a percent of the deviation from the average value of the number reported. For example, an error of $\pm 2 \%$ in a value of 90.0 indicates that the range of error is $88.2 \%$ to $91.8 \%$. The relative error was used to determine the error in two different values evaluated at the same conditions according to Equation 3.3

$$
\text { \% Relative Error }=\left(\frac{\mid \text { Value } 1-\text { Value } 2 \mid}{\left(\frac{\text { Value } 1+\text { Value 2 }}{2}\right)}\right) \times 100
$$




\section{CHAPTER 4 - RESULTS AND DISCUSSION}

This chapter covers the results of the solvent pre-hydrogenation, the parametric studies in the tubing bomb mini-reactors, the one-gallon scale-up digestions and the air blowing treatments. Three of the solvents were hydrogenated at different temperatures and cold hydrogen pressures. For the tubing bomb mini-reactors, the temperature, pressure, and solvent used were all varied, and the best conditions were then used to design the scale-up reactions. After six scale-ups were completed, the digests were then air-blown, all at the same conditions, and all of the products (solvents, hydrogenated solvents, coal-solvent digests, and air-blown digests) were analyzed using the methods listed in Chapter 3. Additionally, the mass balances for the one-gallon scale-up digestions are calculated and these results are used to find the best conditions for this process.

\subsection{Solvent Hydrogenation}

As can be seen from Figures 4.1 - 4.5, the hydrogen pressure in the reactor initially increases with the increasing temperature. However, once a temperature of approximately $250^{\circ} \mathrm{C}$ is reached, the hydrogen pressure begins to drop off sharply, and the final cold pressure of hydrogen was significantly less than the original cold hydrogen pressure in each case. This implies that the catalyst works very well in promoting the hydrogenation of the solvent. The mass of $\mathrm{H}_{2}$ gas reacted with the solvent and the weight

percent of $\mathrm{H}_{2}$ added for each run is in Table 4.1. While these results are compelling, it is important to note that only a small amount of hydrogen has actually been added to any of 
the solvents (maximum of $\sim 0.25 \% \mathrm{wt}$.), a consequence of this process being much milder than most of the research noted in the Section 2. The low level of hydrogenation could imply that the aromatic components were not totally saturated and that concentration of hydroaromatics was increased, which is desirable. This helps to keep the economical and safety concerns for the use of hydrogen gas in this process at a minimum. Hydrogen consumption was calculated using the initial and final cold pressure in the reactor (assuming hydrogen only in the vapor phase) with the ideal gas equation (Eqn. 4.1) to obtain the initial and final mass of hydrogen gas present, then finding the difference.

$$
\frac{\mathrm{P}_{\text {init }} \times \mathrm{V}_{\text {init }}}{\text { Mass }_{\text {init }} \times \mathrm{T}_{\text {init }}}=\frac{\mathrm{P}_{\text {final }} \times \mathrm{V}_{\text {final }}}{\text { Mass }_{\text {final }} \times \mathrm{T}_{\text {final }}}
$$

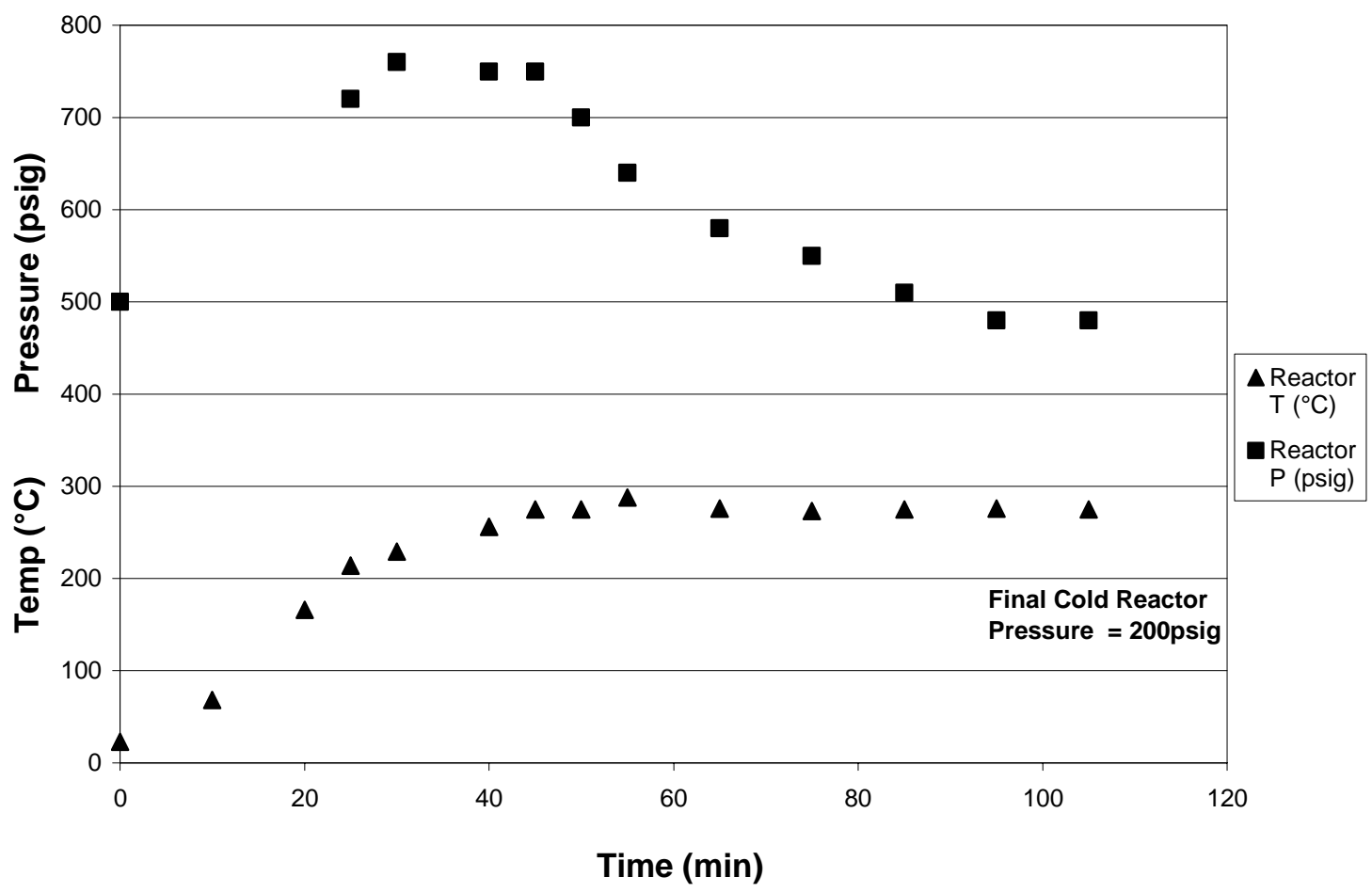

Figure 4.1 - Temperature and Pressure for HCBB Level 1 Hydrogenation 


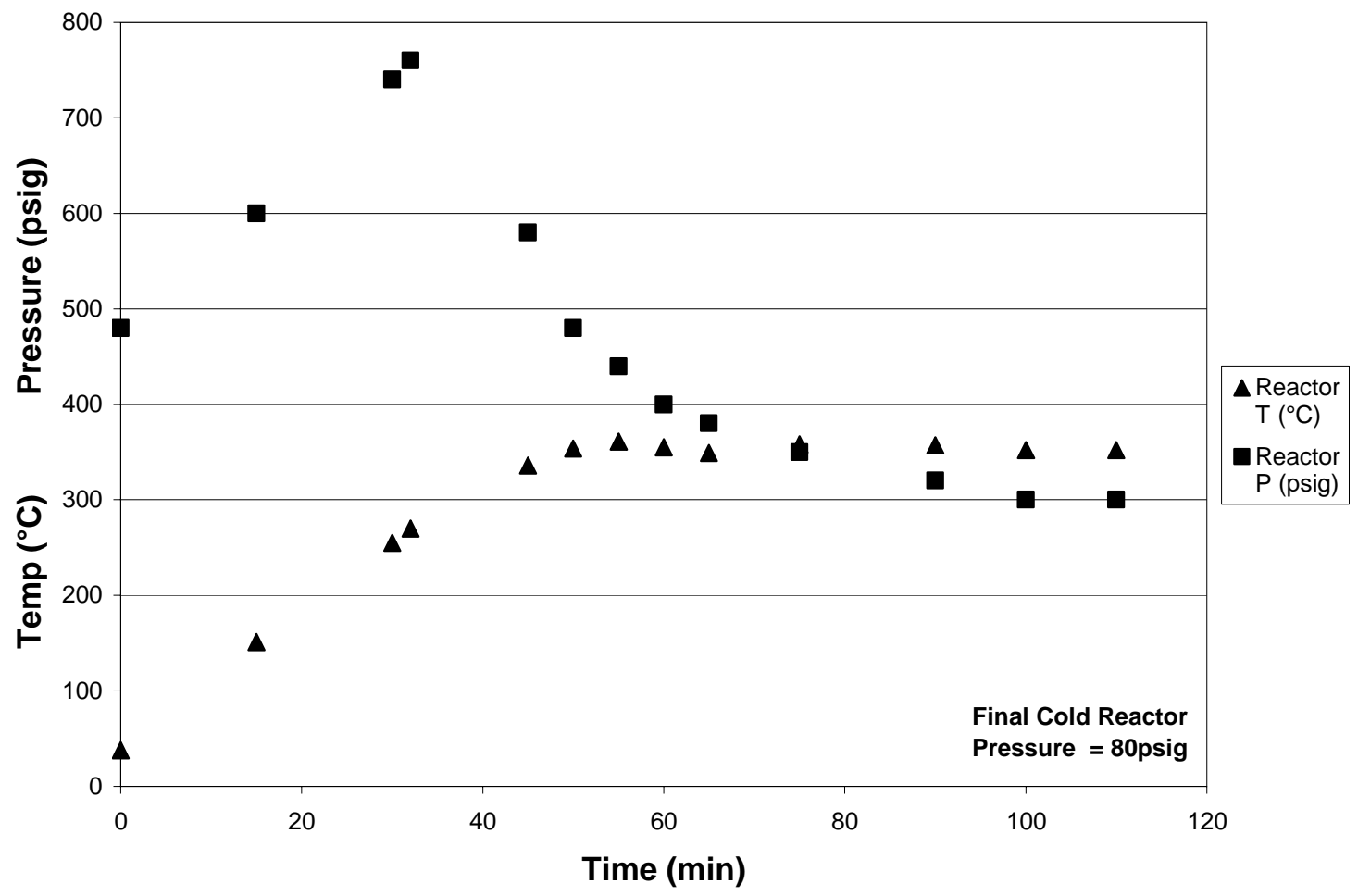

Figure 4.2 - Temperature and Pressure for HCBB Level 2 Hydrogenation

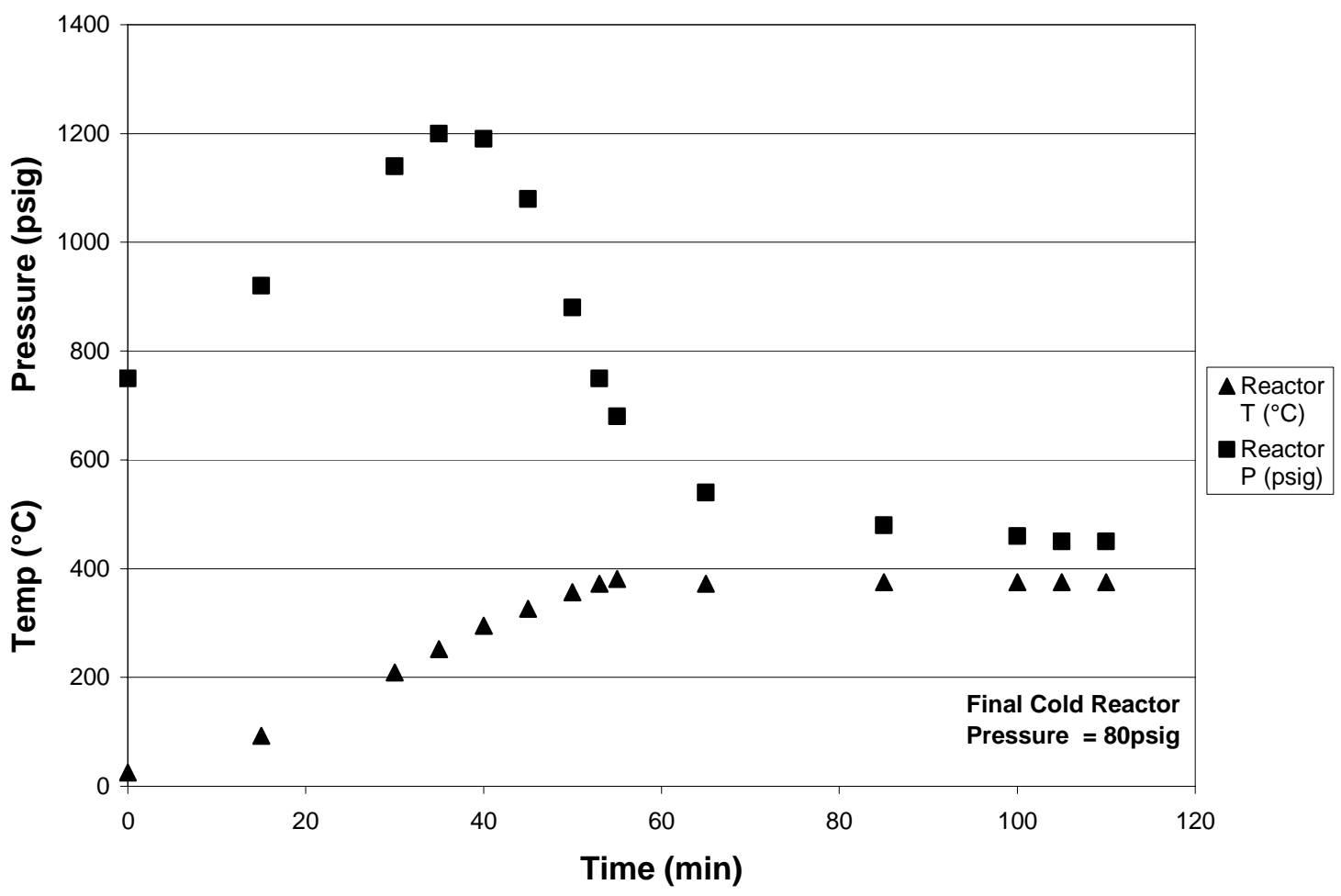

Figure 4.3 - Temperature and Pressure for HCBB Level 3 Hydrogenation 


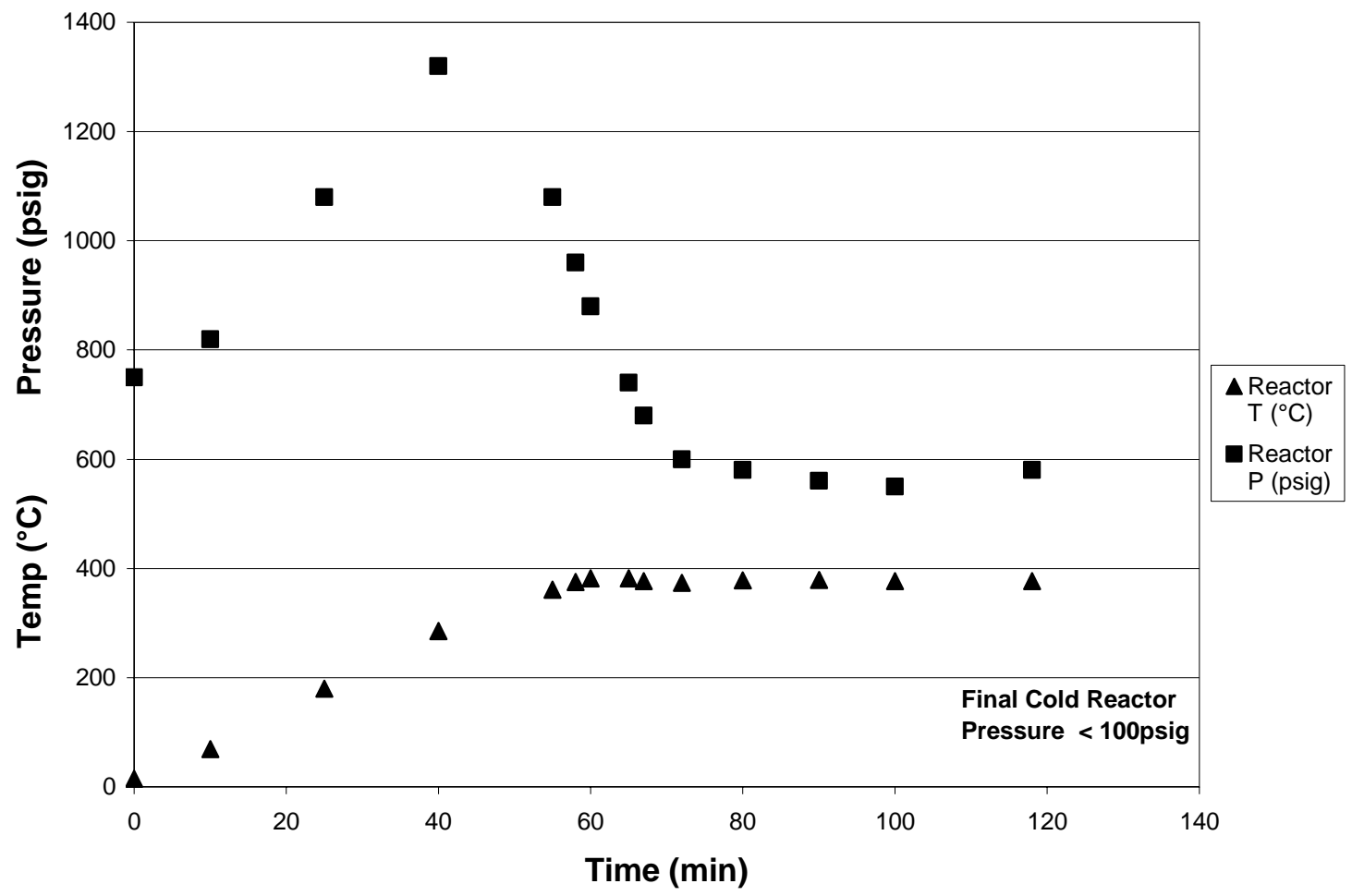

Figure 4.4 - Temperature and Pressure for HSO Level 3 Hydrogenation

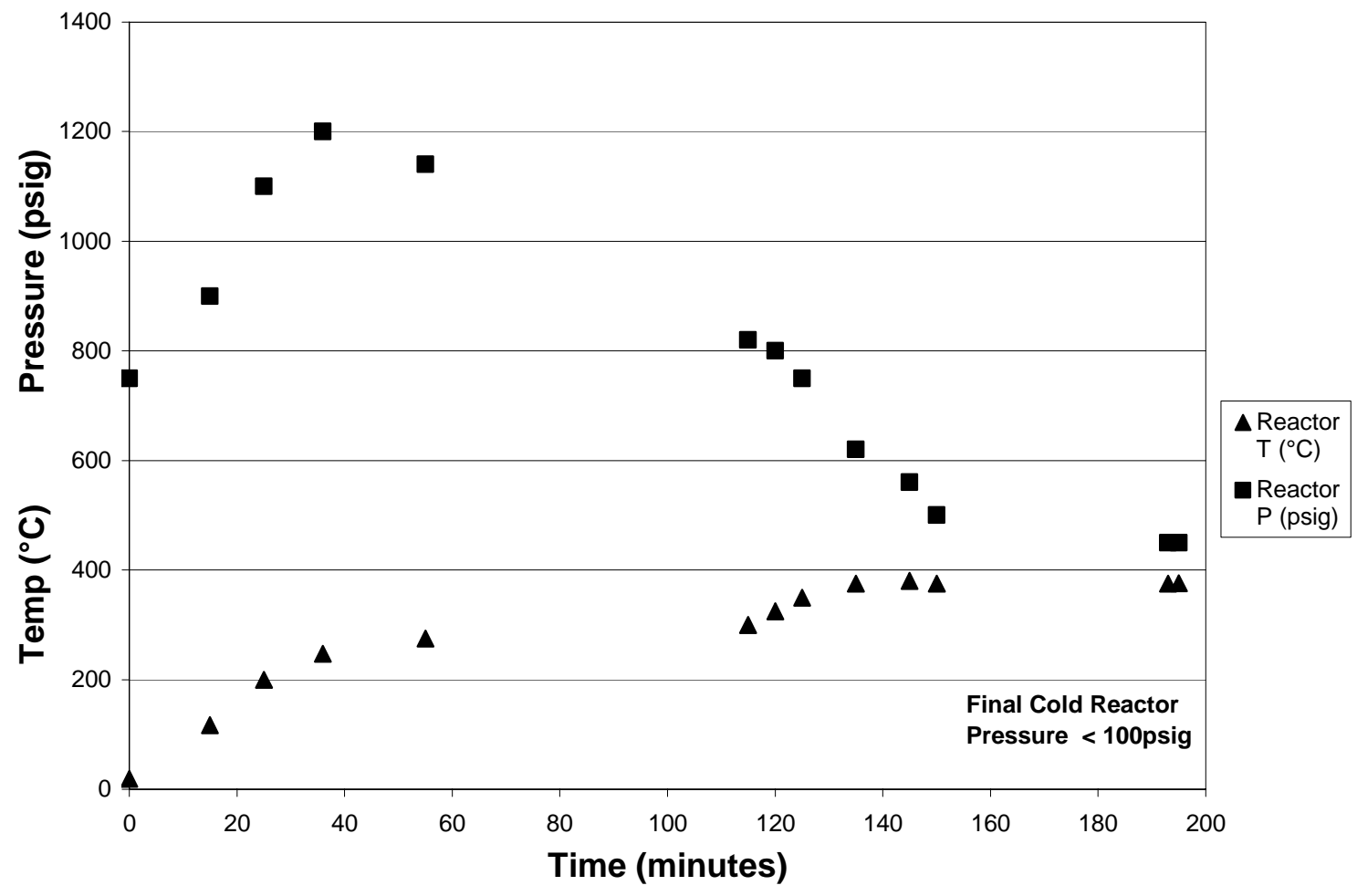

Figure 4.5 - Temperature and Pressure for HMO Level 3 Hydrogenation 
Table 4.1 - Solvent Hydrogenation Results

\begin{tabular}{|c|c|c|c|c|}
\hline Run Description & $\begin{array}{c}\text { Mass H2 } \\
\text { absorbed } \\
\mathbf{( g )}\end{array}$ & $\begin{array}{c}\text { Wt\% H2 } \\
\text { absorbed }\end{array}$ & $\begin{array}{c}\text { Reactor } \\
\mathbf{T},{ }^{\circ} \mathbf{C}\end{array}$ & $\begin{array}{c}\text { Initial cold } \\
\text { H2 Pressure } \\
\text { (psig) }\end{array}$ \\
\hline $\begin{array}{c}\text { CBB Hydrogenation } \\
\text { Level 1 }\end{array}$ & 12.99 & 0.10 & 275 & 500 \\
\hline $\begin{array}{c}\text { CBB Hydrogenation } \\
\text { Level 2 }\end{array}$ & 16.17 & 0.14 & 350 & 500 \\
\hline $\begin{array}{c}\text { CBB Hydrogenation } \\
\text { Level 3 }\end{array}$ & 28.48 & 0.24 & 375 & 750 \\
\hline $\begin{array}{c}\text { Slurry Oil } \\
\text { Hydrogenation Level } \\
3\end{array}$ & 28.64 & 0.24 & 375 & 750 \\
\hline $\begin{array}{c}\text { Maraflex Oil } \\
\text { Hydrogenation Level } \\
3\end{array}$ & 28.20 & 0.24 & 375 & 750 \\
\hline
\end{tabular}

Simulated Distillation performed on each of the solvents used in this study shows some distinct changes in the boiling point distributions with hydrogenation. Comparing the raw solvents against each other (Figure 4.6), one can see the smooth and broad distribution present with the petroleum-derived solvents. The carbon black base (CBB) shows a more narrow distribution range, but a much more erratic curve. This is the result of the fact that this solvent is composed of distinct classes of aromatics, the steps in the curve possibly related to the ring numbers. For example, naphthalene is believed to cause the pastiness the solvent exhibits at room temperature. With hydrogenation, each solvent's boiling point distribution shifts, as the compounds become slightly more saturated with hydrogen, or, in the case of the slurry oil, the molecules lose some small aliphatic side chains. Each solvent responds to the hydrogenation, but the carbon black 
base responds in a more significant way, as there is not just a shift, but a substantial narrowing of the boiling point range, especially for the level 3 hydrogenated carbon black base, HCBB-L3 (Figure 4.7-9).

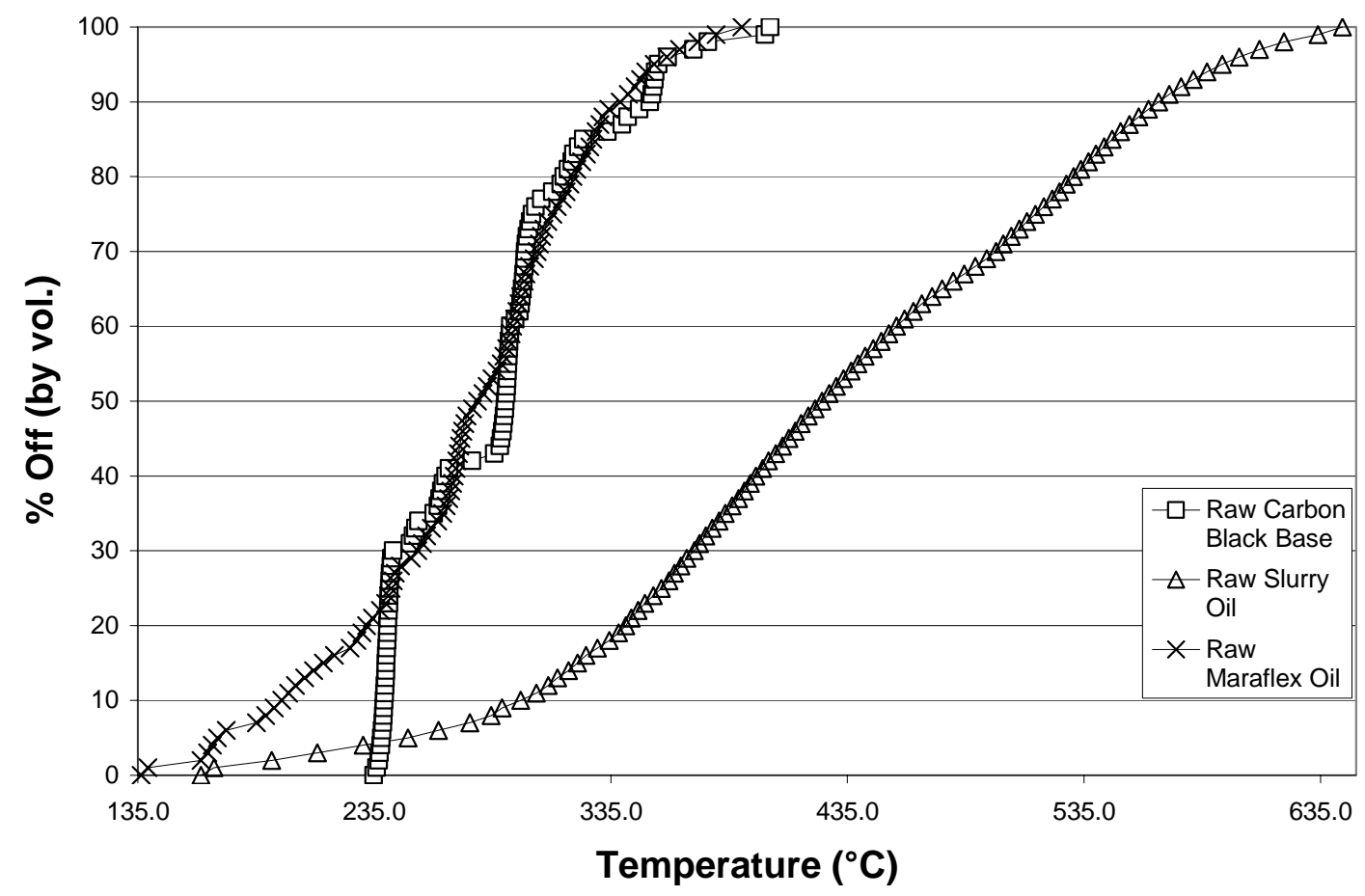

Figure 4.6 - Boiling Point Curves of Raw Solvents 


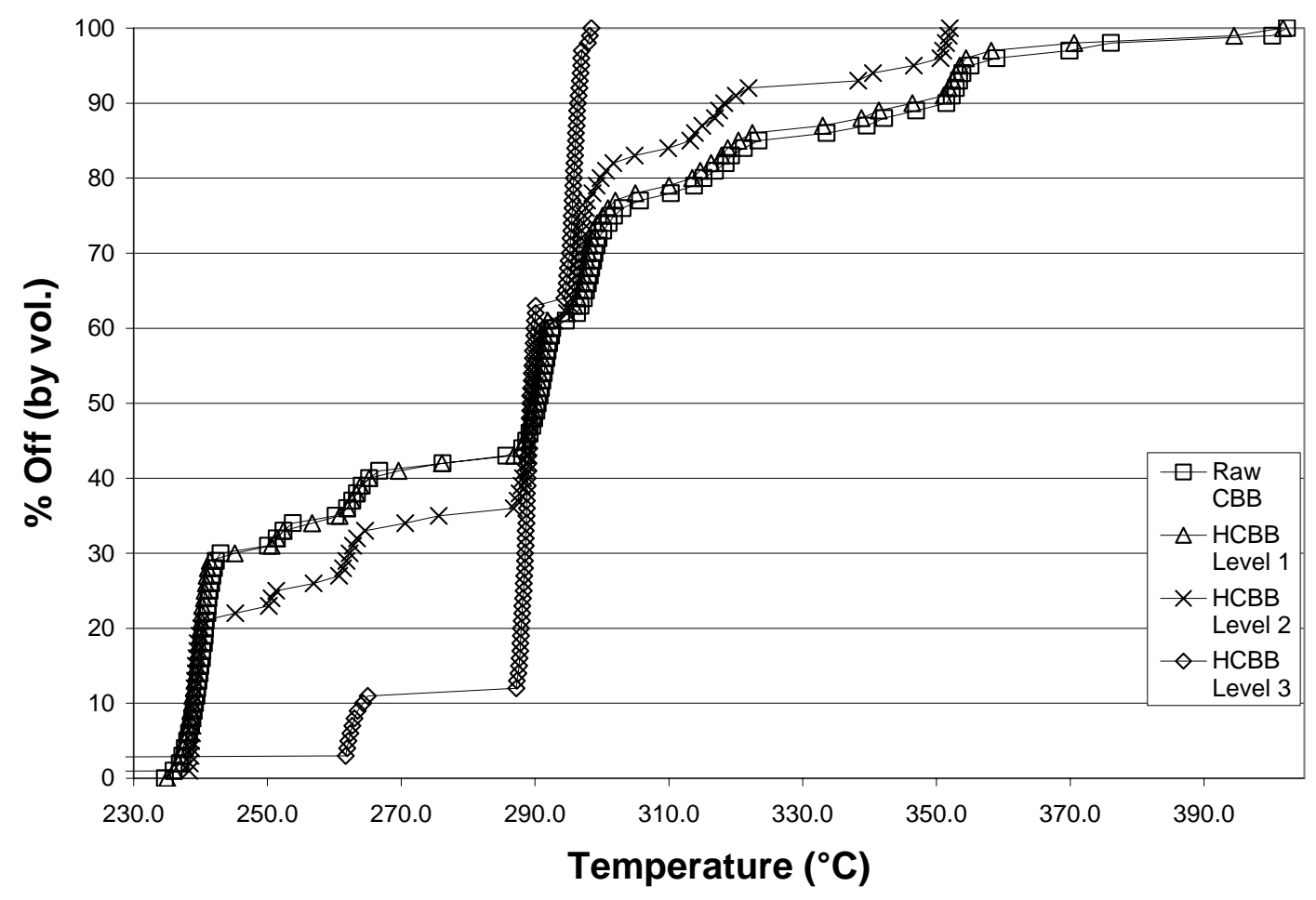

Figure 4.7 - Boiling Point Curves of CBB solvents

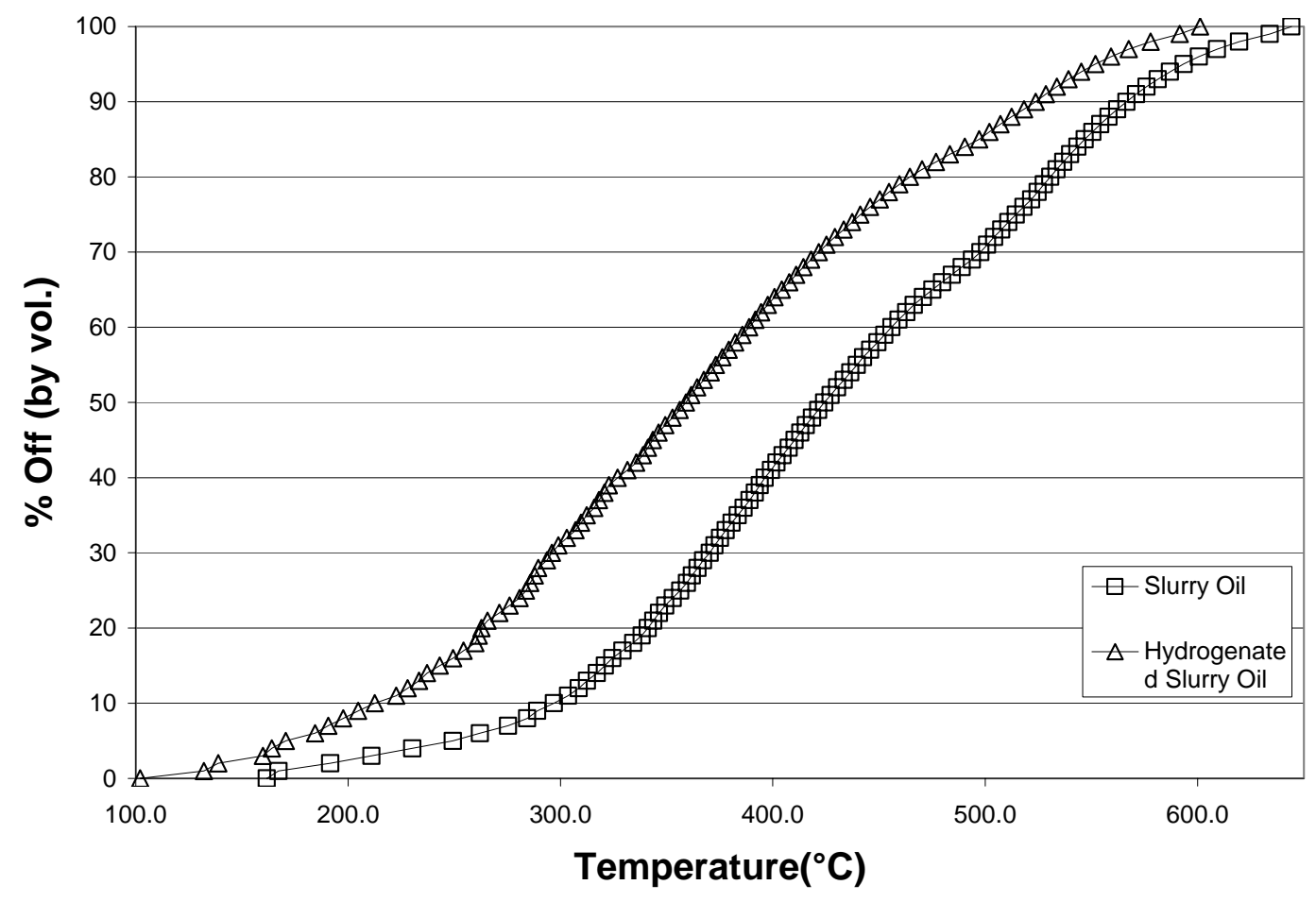

Figure 4.8 - Boiling Point Curves of Slurry Oils 


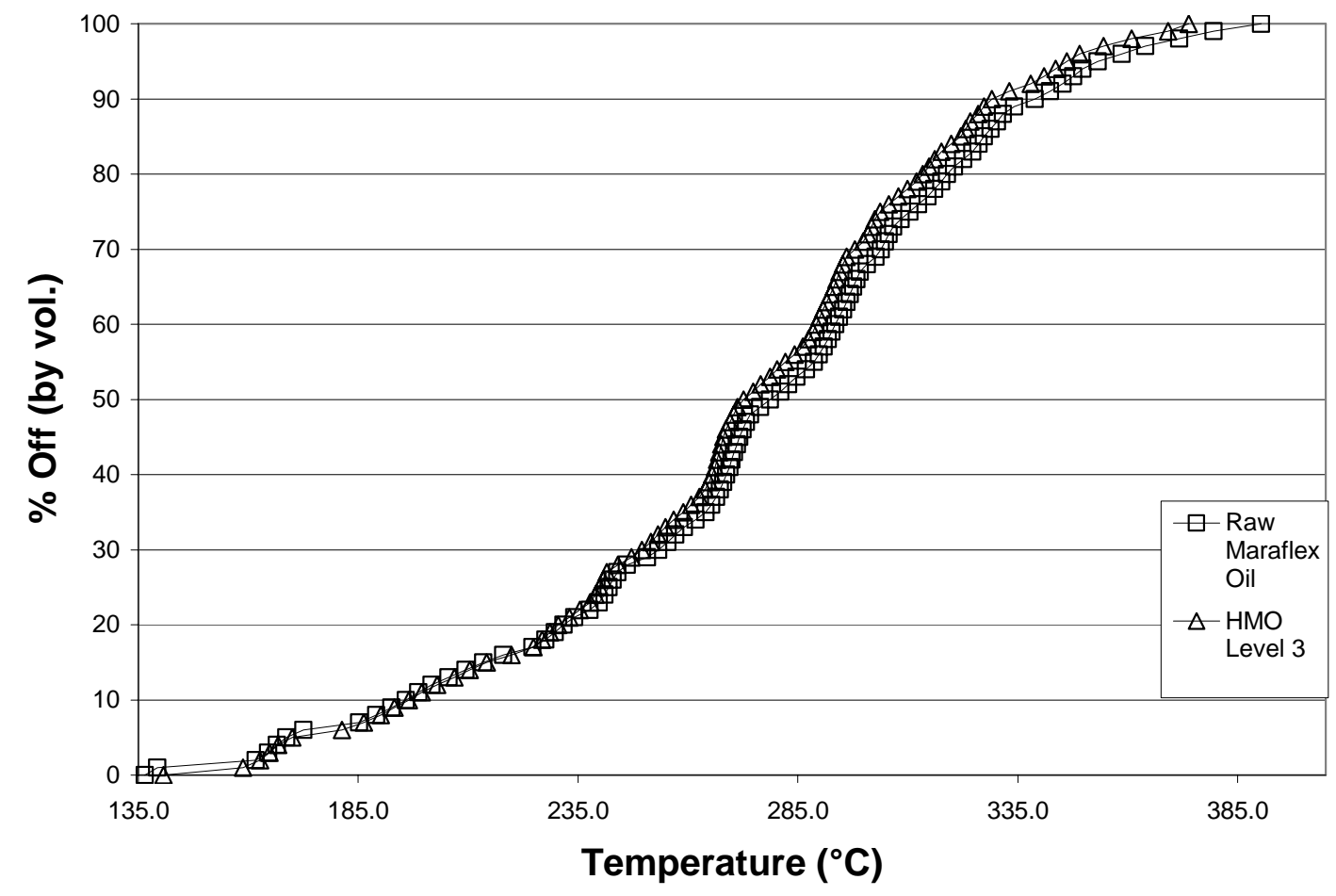

Figure 4.9 - Boiling Point Curves of Maraflex Oils

The first derivative of the boiling point curves was calculated using a simple slope calculation at each point in the curve (rise / run). Each curve shows the rate of change of the boiling point curves, and these were prepared in an effort to see if there is some pattern in the curves for the different solvents. While it is apparent from comparing the curves for the slurry oil and Maraflex oil versus their hydrogenated counterparts, respectively, that a change has taken place (often as significant change), it is not apparent how this might apply to the present study. It is interesting to note, though, that the changes with the carbon black base curves, much like the original boiling point distributions themselves, are erratic and, in fact, the level 3 hydrogenation curve is extremely different from the other CBB curves (Figures 4.10-17). This curve has many points, especially at higher temperatures, where the derivative is infinity (division by 0), 
but to keep this chart readable, the derivative marked as unchanged from the previous point. The highest peaks in the derivative (corresponding to the largest rate of change), all occur at 10 for the CBB samples; the placement of these peaks simply shifts to higher temperatures. For the slurry oil, the height of the highest peaks increases with hydrogenation, indicating a faster rate of change of the boiling point in the range of 350$400^{\circ} \mathrm{C}$. For the Maraflex oil, the fastest rate of change of the boiling point curves occurs at a different temperature in the raw solvent than in the hydrogenated solvent.

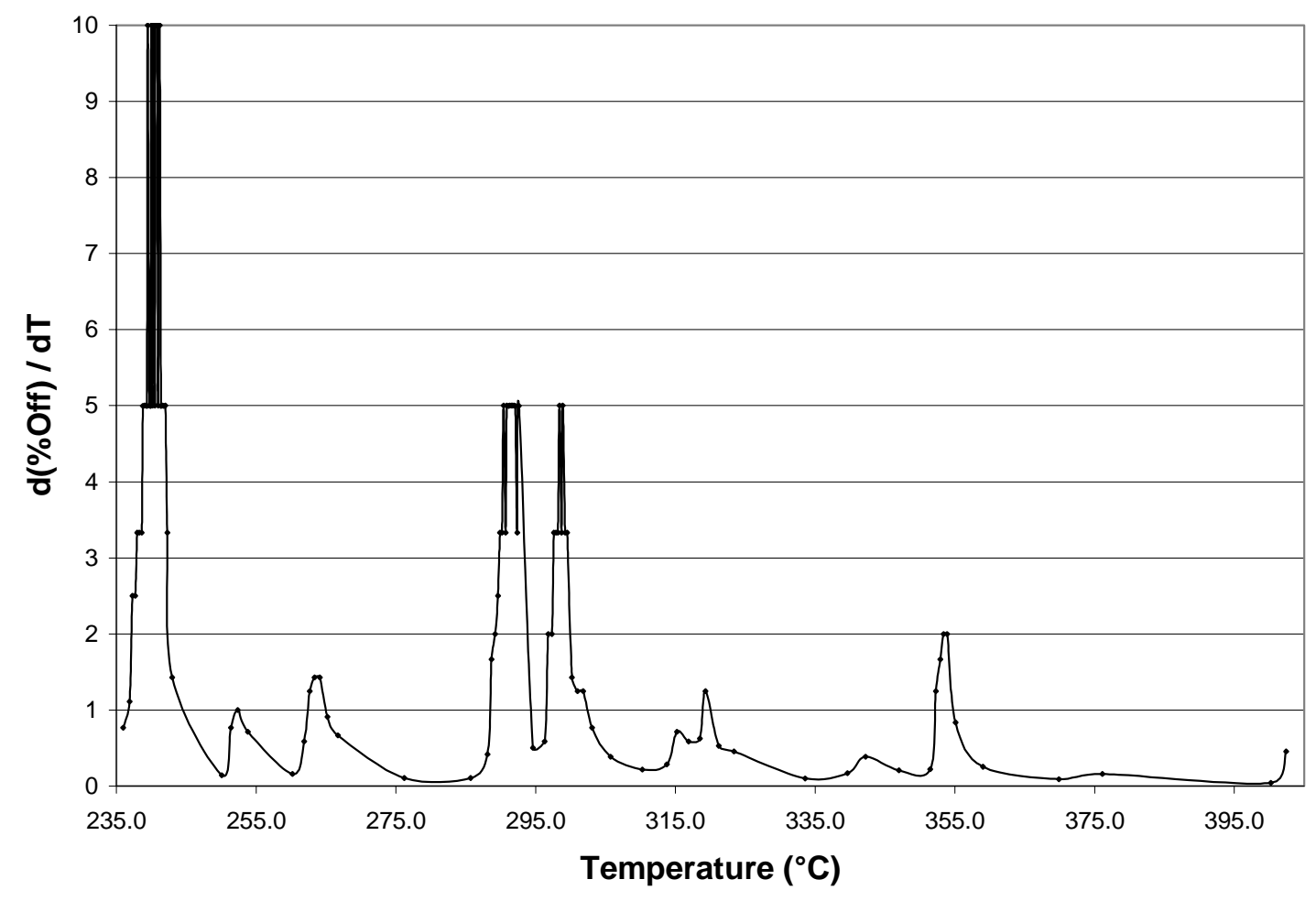

Figure 4.10 - First Derivative of Boiling Point Curve for CBB vs Temperature 


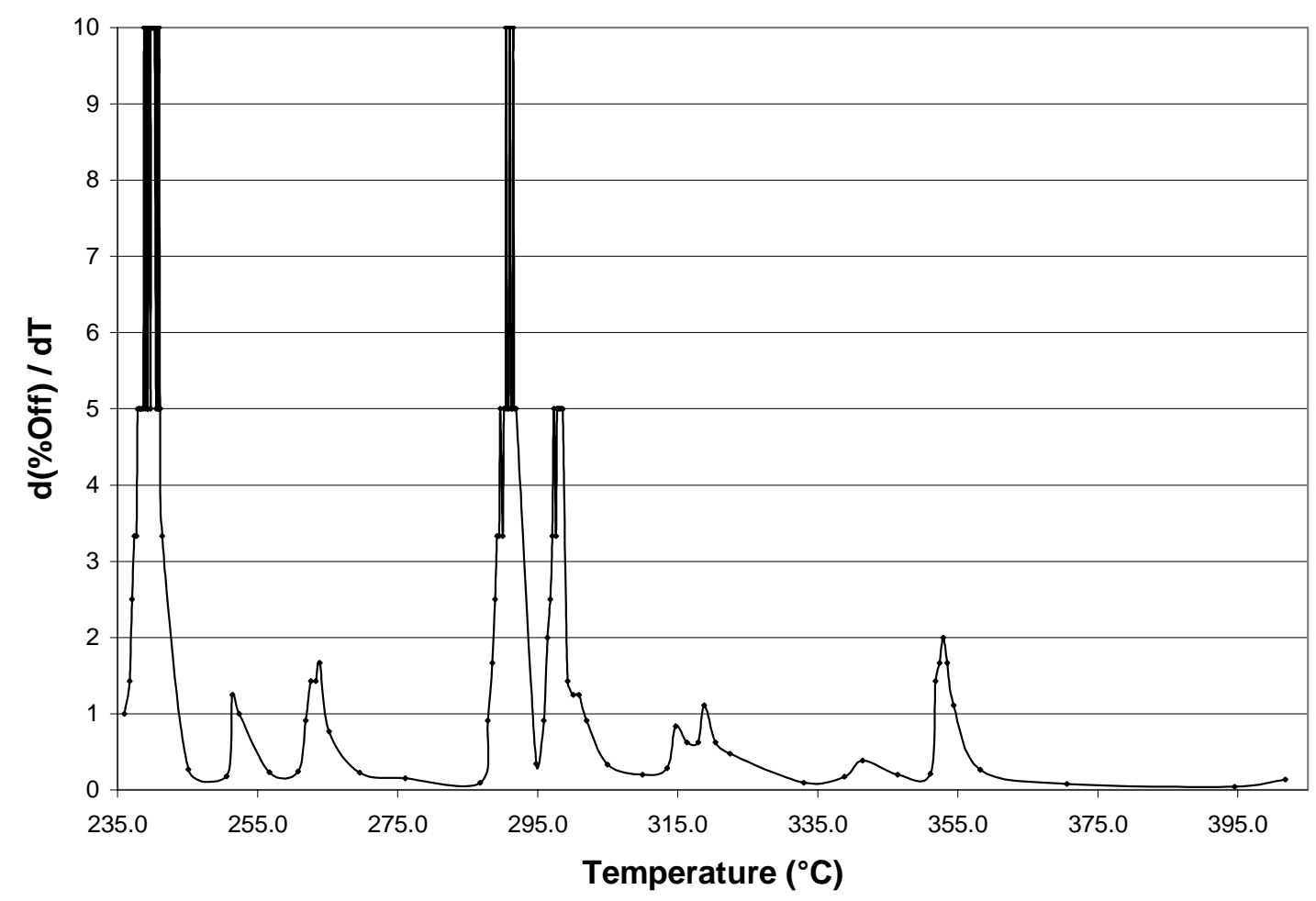

Figure 4.11 - First Derivative of Boiling Point Curve for HCBB-L1 vs Temperature

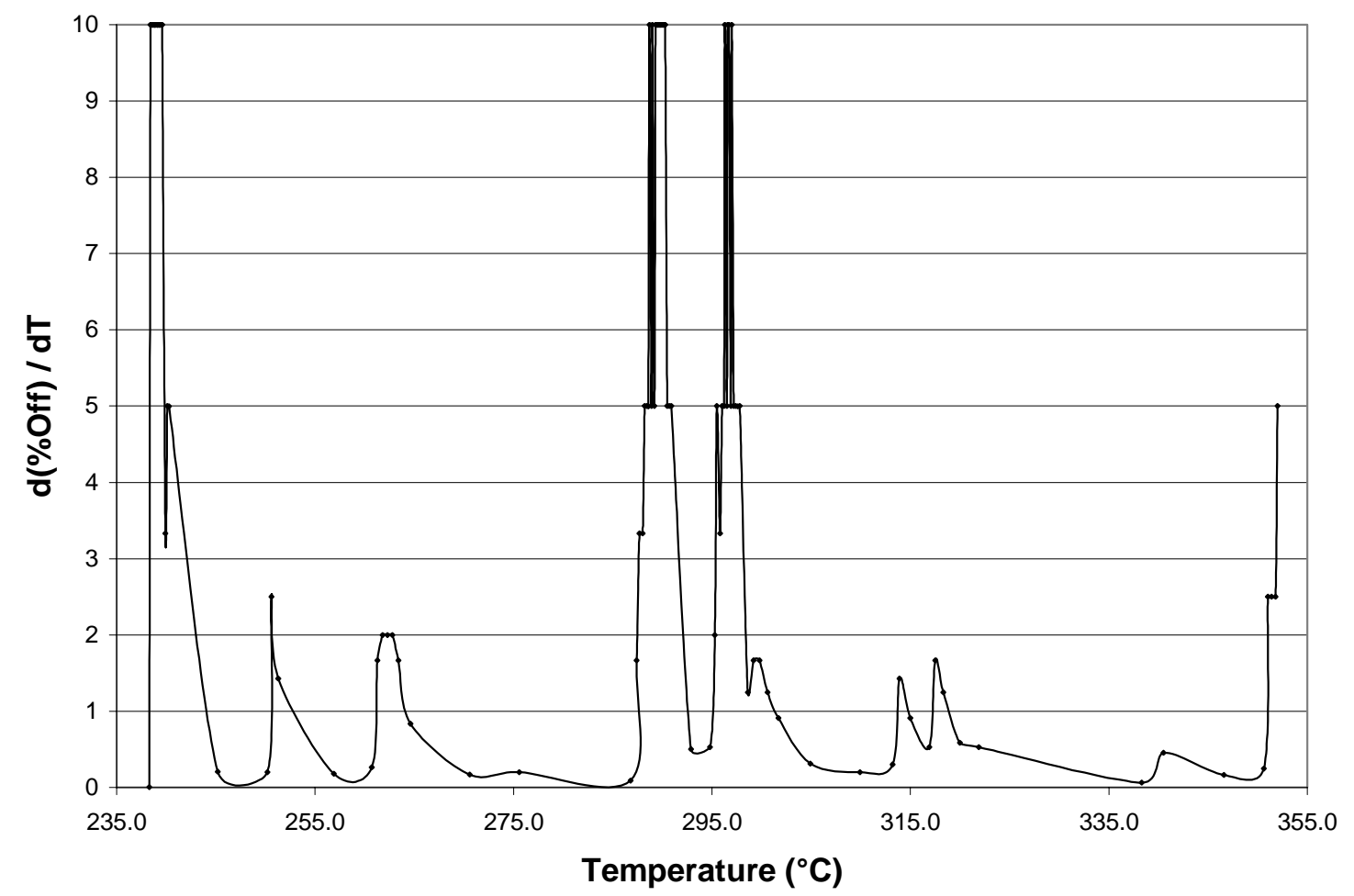

Figure 4.12 - First Derivative of Boiling Point Curve for HCBB-L2 vs Temperature 


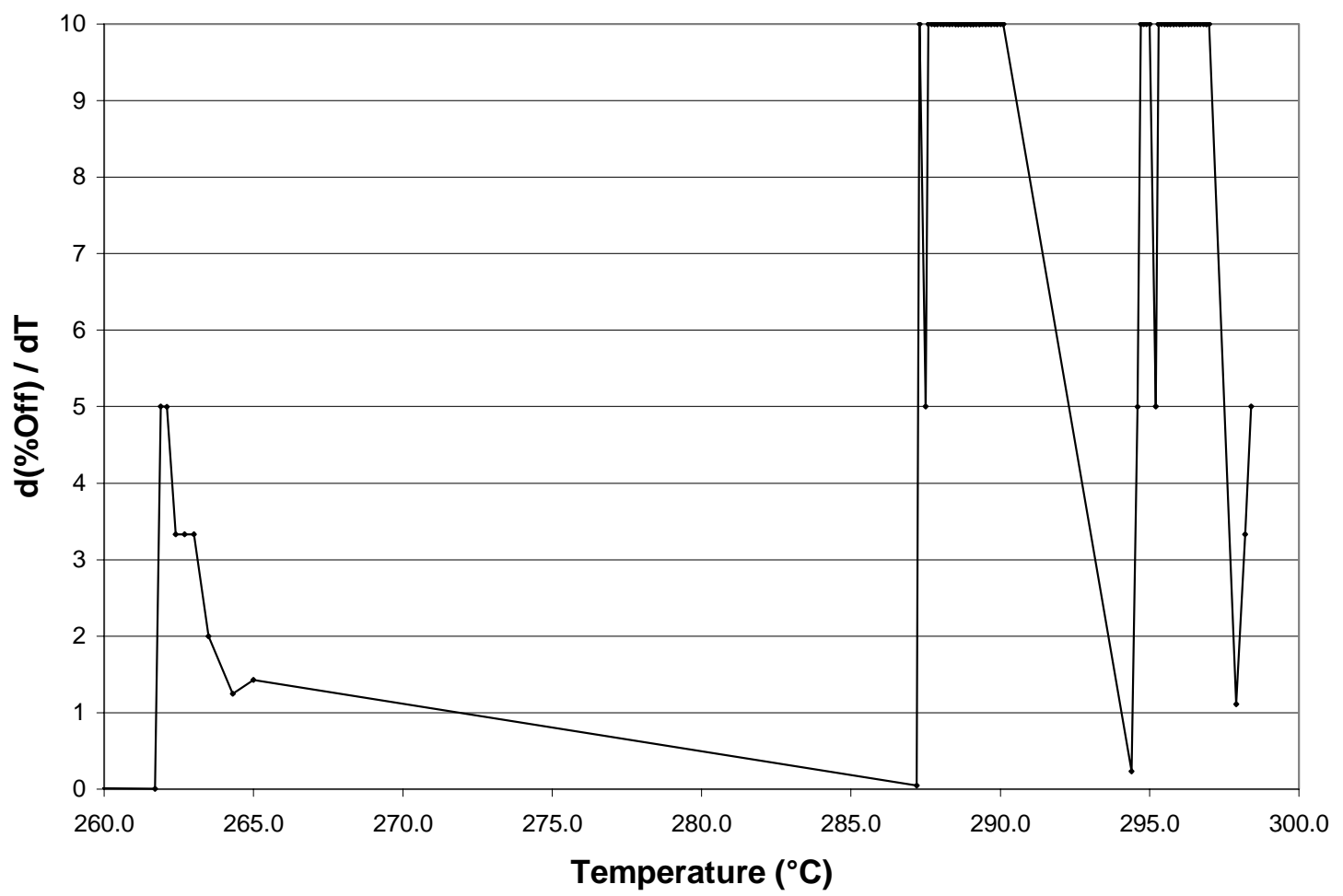

Figure 4.13 - First Derivative of Boiling Point Curve for HCBB-L3 vs Temperature

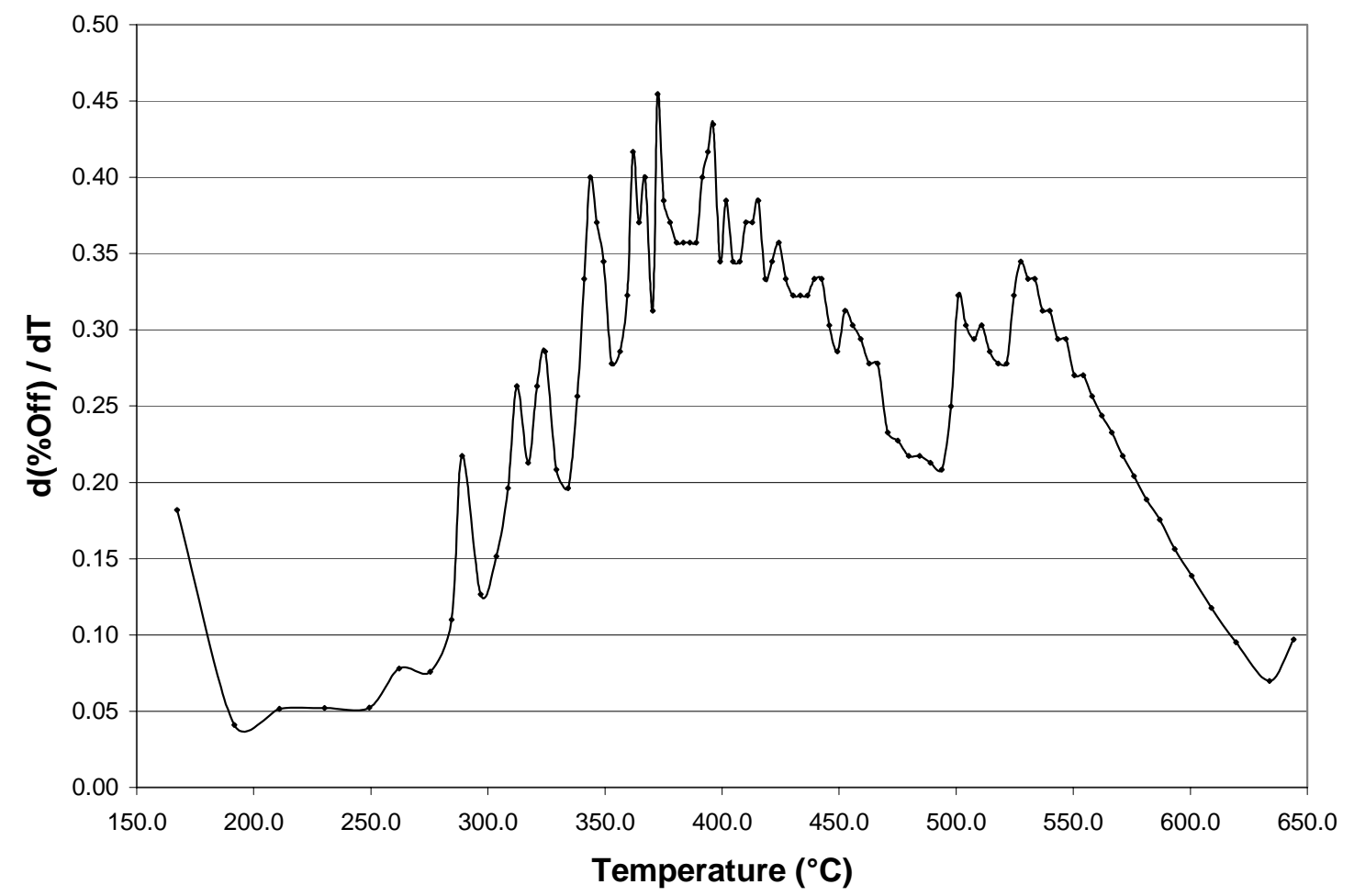

Figure 4.14 - First Derivative of Boiling Point Curve for Slurry Oil vs Temperature 


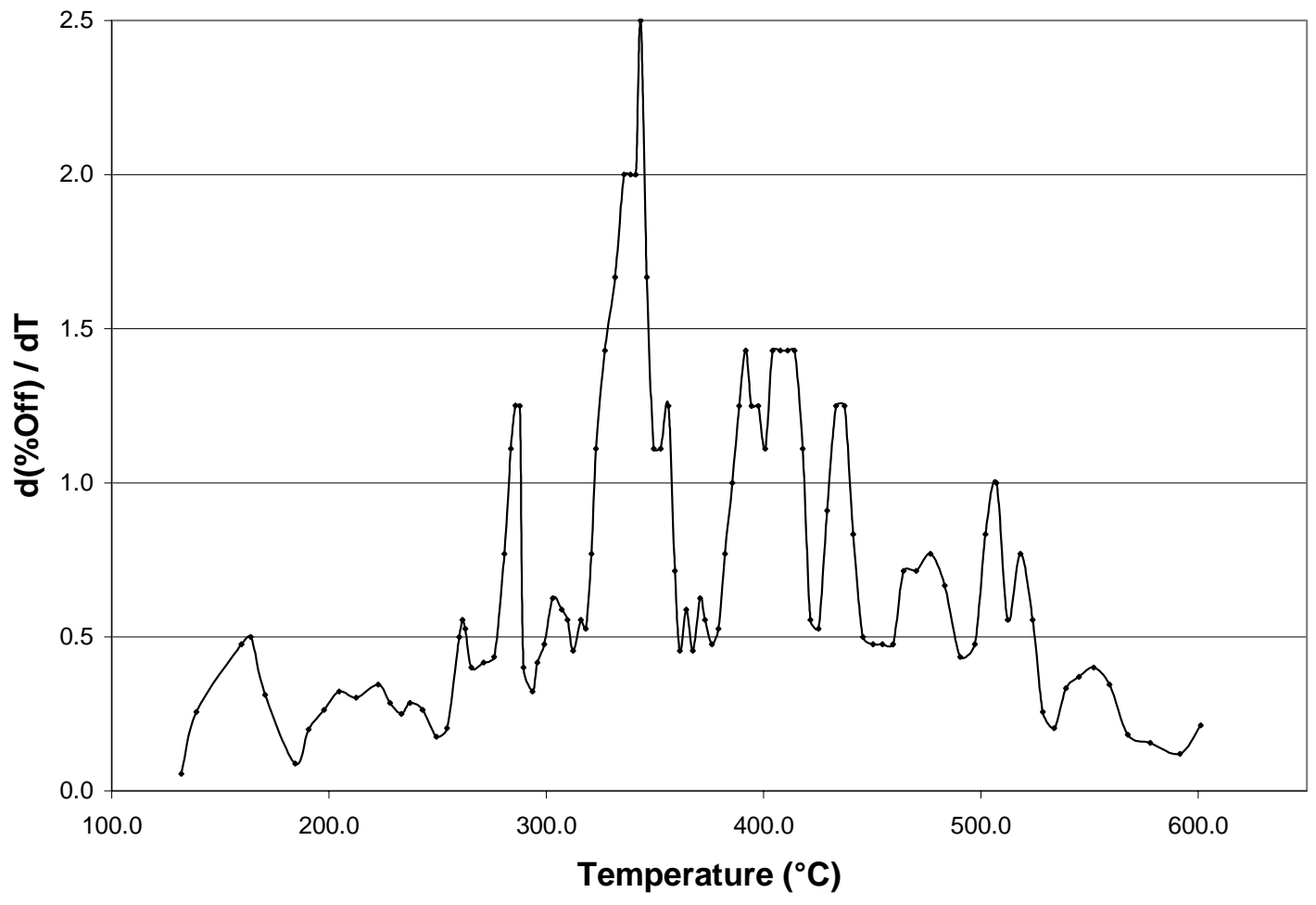

Figure 4.15 - First Derivative of Boiling Point Curve for HSO-L3 vs Temperature

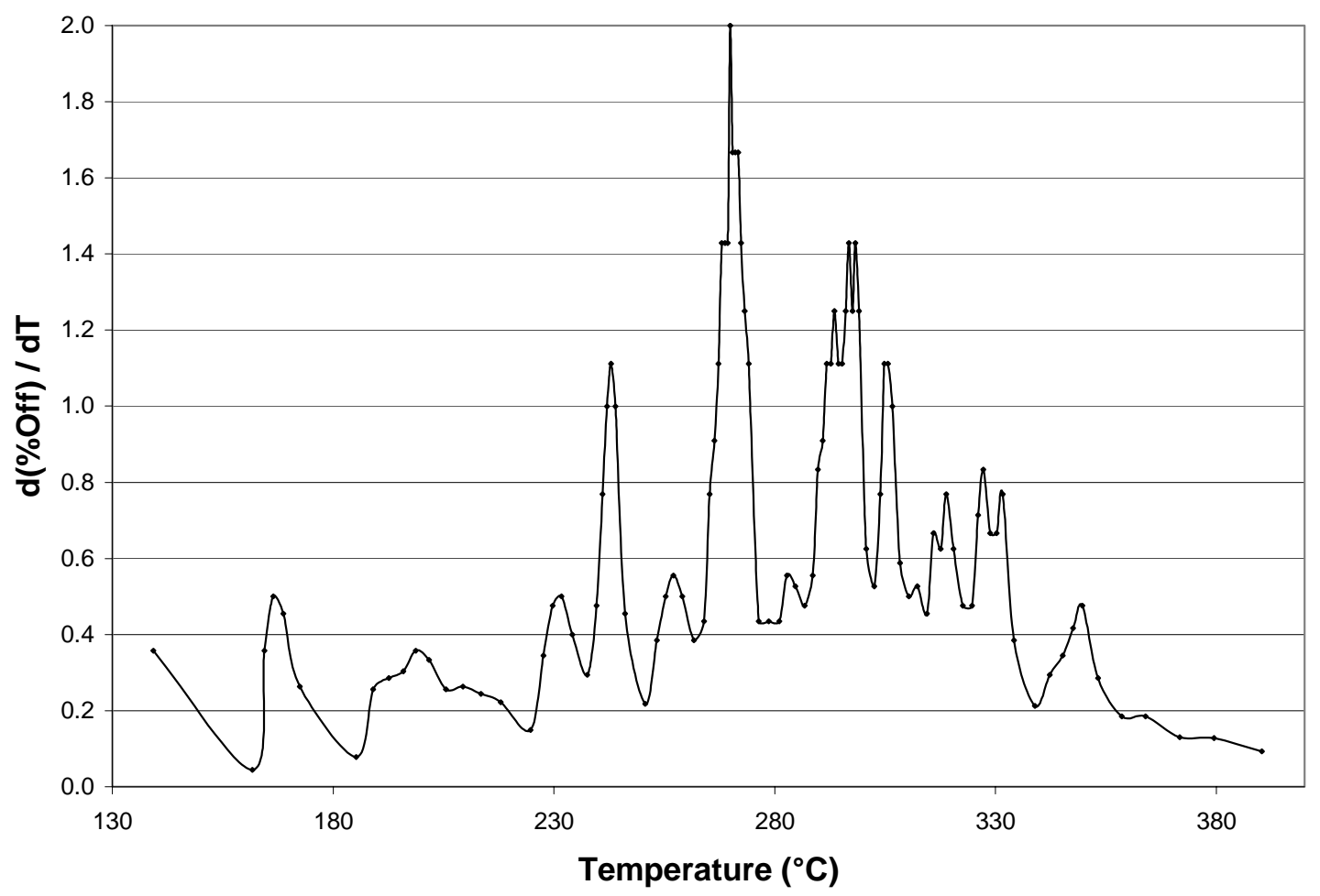

Figure 4.16 - First Derivative of Boiling Point Curve for Maraflex Oil vs Temperature 


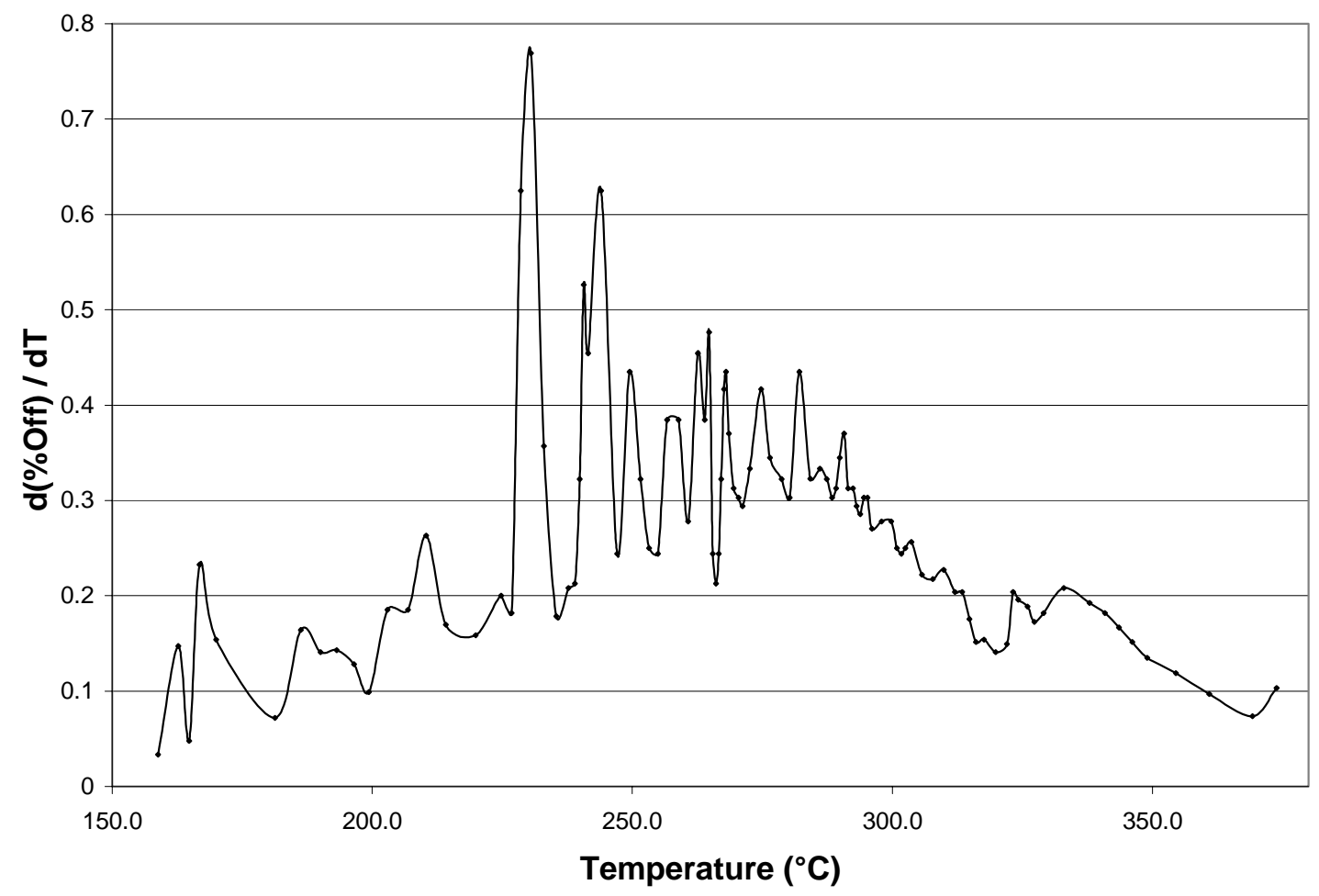

Figure 4.17 - First Derivative of Boiling Point Curve for HMO-L3 vs Temperature

Elemental Analysis and FTIR were also performed on each of the solvents and hydrogenated variant. These results will be looked at in much greater detail later in this section, but the $\mathrm{C} / \mathrm{H}$ ratio of the solvents used in this study, calculated from the elemental analysis results, do give an indication of what is happening in the solvent hydrogenation (Table 4.2). The first observation that can be made is the obvious difference in $\mathrm{C} / \mathrm{H}$ ratios of the coal derived liquids $(\mathrm{CBB}, \mathrm{AO})$ and the petroleum derived liquids (SO, MO). In both the carbon black base and Maraflex oil samples, as the hydrogenation level increases, the $\mathrm{C} / \mathrm{H}$ atomic ratio and aromaticity factor $\left\{\mathrm{H}_{\mathrm{ar}} /\left(\mathrm{H}_{\mathrm{ar}}+\mathrm{H}_{\mathrm{al}}\right)\right\}$ decrease, indicating that hydrogen is added to the solvents and is saturating the ring structures therein. However, for the slurry oil, the $\mathrm{C} / \mathrm{H}$ ratio and aromaticity factor increases with hydrogenation. During this reaction, it is hypothesized that in addition to hydrogen being 
added in bulk to the slurry oil, that perhaps small aliphatic side chains (i.e. ethane, propane) are being broken off of the solvent molecules and released into the vapor phase. Gas chromatographic analysis of the off-gas from the solvent hydrogenation would corroborate this theory, and samples were taken, but this analysis was not available during this thesis study.

Table 4.2 - Elemental and FTIR Analysis Data for Solvents

\begin{tabular}{|c|ccc|ccc|}
\hline Solvent & \% C & \% H & $\begin{array}{c}\text { C I H atomic } \\
\text { ratio }\end{array}$ & Har & Hal & $\begin{array}{c}\mathbf{H}_{\text {ar }} \mathbf{l} \\
\left(\mathbf{H}_{\text {ar }}+\mathbf{H}_{\text {al }}\right)\end{array}$ \\
\hline CBB & 91.58 & 5.71 & 1.336 & 73.77 & 50.51 & 0.594 \\
HCBB-L1 & 92.15 & 5.81 & 1.322 & 75.11 & 57.98 & 0.564 \\
HCBB-L2 & 91.97 & 5.82 & 1.316 & 70.71 & 58.57 & 0.547 \\
HCBB-L3 & 91.39 & 5.84 & 1.303 & 74.91 & 70.97 & 0.514 \\
SO & 87.38 & 9.56 & 0.762 & 23.39 & 362.55 & 0.061 \\
HSO-L3 & 87.13 & 8.98 & 0.808 & 28.18 & 295.39 & 0.087 \\
MO & 92.22 & 7.77 & 0.990 & 56.74 & 144.03 & 0.283 \\
HMO-L3 & 92.19 & 7.82 & 0.982 & 51.11 & 155.58 & 0.247 \\
AO & 92.41 & 6.21 & 1.241 & N/A & N/A & N/A \\
\hline
\end{tabular}

Additionally, the amount of sulfur (S) present in the solvents is affected in this hydrogenation step. The amount of sulfur in all of the solvents is decreased with hydrogenation, as can be seen from Figure 4.18. This is good, especially for the slurry oils, which contain a larger amount of sulfur than any of the other solvents initially. The nitrogen level in these solvents is not appreciably affected in the hydrogenation, but the level is reasonably low in this process, and the nitrogen level in the products is not of importance in this study. 


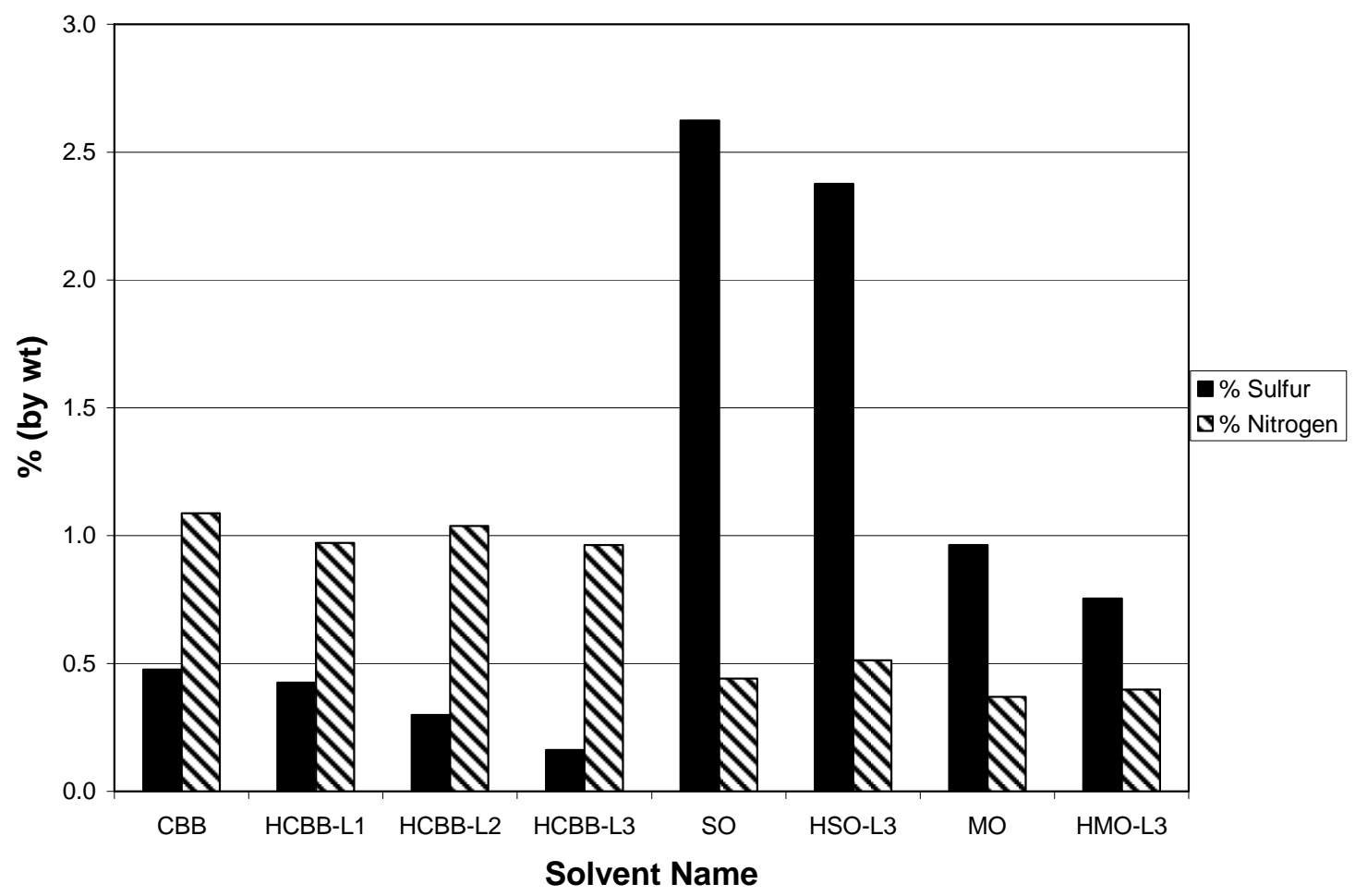

Figure 4.18 - Amount of Sulfur and Nitrogen (by wt \%) in Solvents

\subsection{Parametric Studies in Mini Reactors}

From the tubing bomb reactions, the coal conversions obtained indicate that there is an optimal temperature and pressure at which to conduct the scale-up reactions. As can be seen in Figure 4.19 and 4.20, the optimal conversion is achieved at $400^{\circ} \mathrm{C}$. The low conversion obtained at lower temperatures is due to an inability of the solvent to thoroughly digest the coal, while the lower conversion achieved at higher temperatures is likely due to retrograde reactions (polymerizations, combinations) that occur at elevated temperatures, at or near $450^{\circ} \mathrm{C}$. Note also that the maximum digestion conversions follow the level of hydrogenation. 


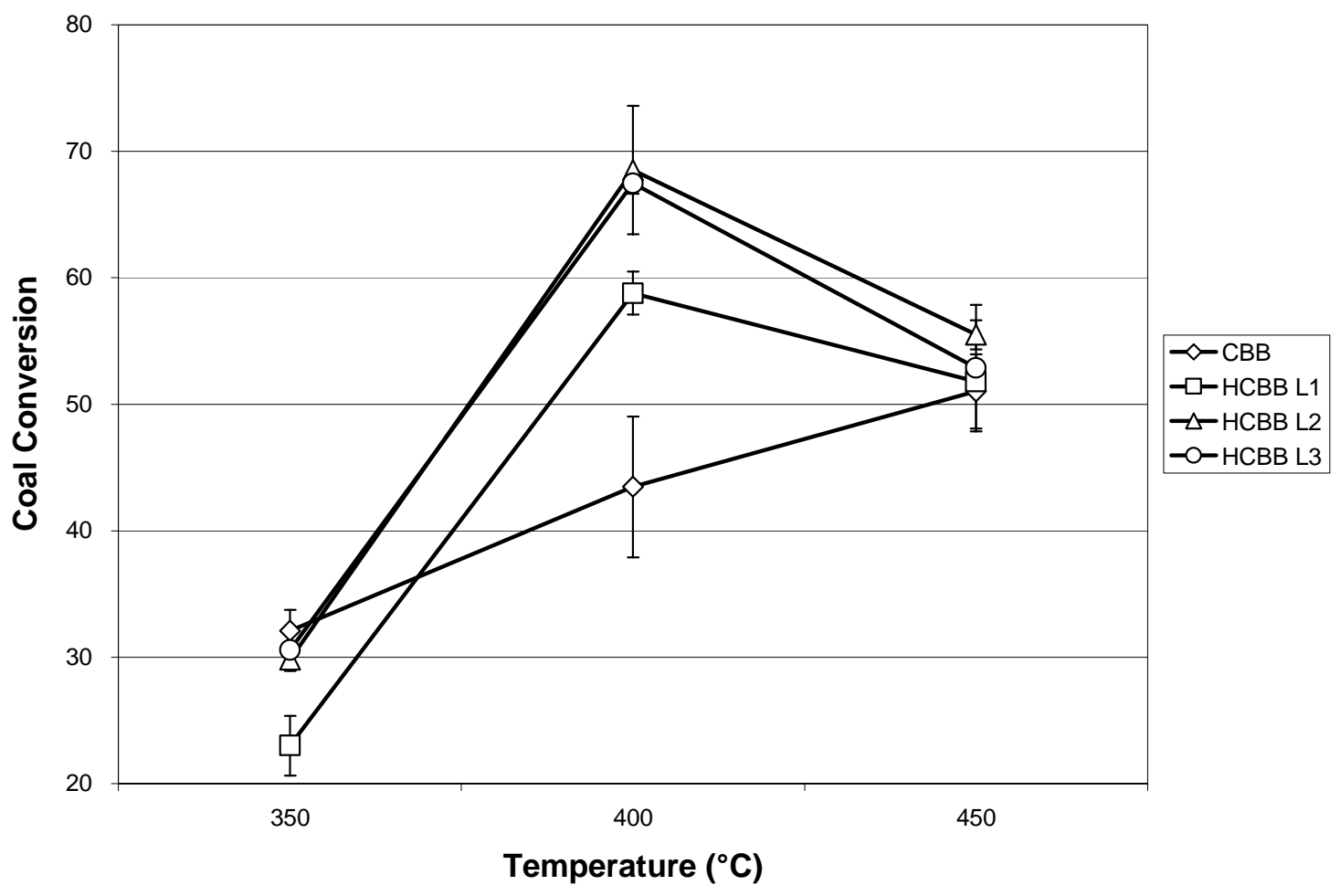

Figure 4.19 - Conversion vs. Temperature at $P=0$ psig $N 2$

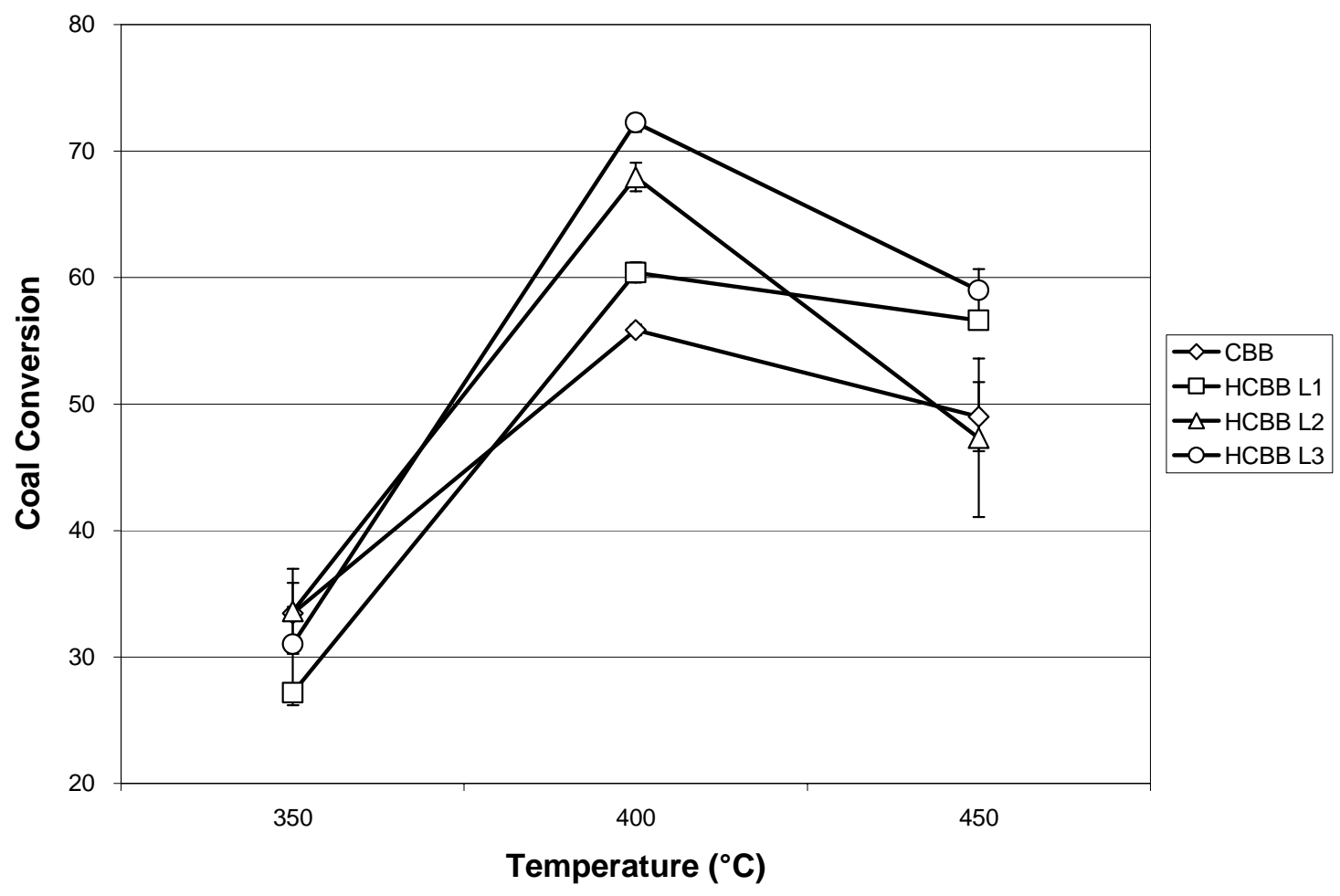

Figure 4.20 - Conversion vs. Temperature at $P=500$ psig N2 
In the determination of the best solvent or solvent combination to use in the scaleup reactions, the first set of data to observe is the conversion of coal in each of the raw solvents as compared to tetralin, which was used for comparison as a standard H-donor liquefaction solvent (Figure 4.21). It can be seen that the CBB works the best compared to tetralin, followed by the slurry oil, Maraflex oil and then the anthracene oil. While the anthracene oil is widely reported in the literature to be one of the better industrial byproduct oils for solvent digestion of coal, those results were typically at far more severe conditions, and typically the anthracene oil had already been pretreated. The anthracene oil used in this study contained visible solids and may not have been the exact same material as the anthracene oil used in other studies. It is, however, theorized here that the anthracene oil, if properly hydrogenated, would be an adequate digestion solvent for this type of process. However, as the anthracene oil (AO) performed so poorly, it was eliminated from any further research and discussion. 


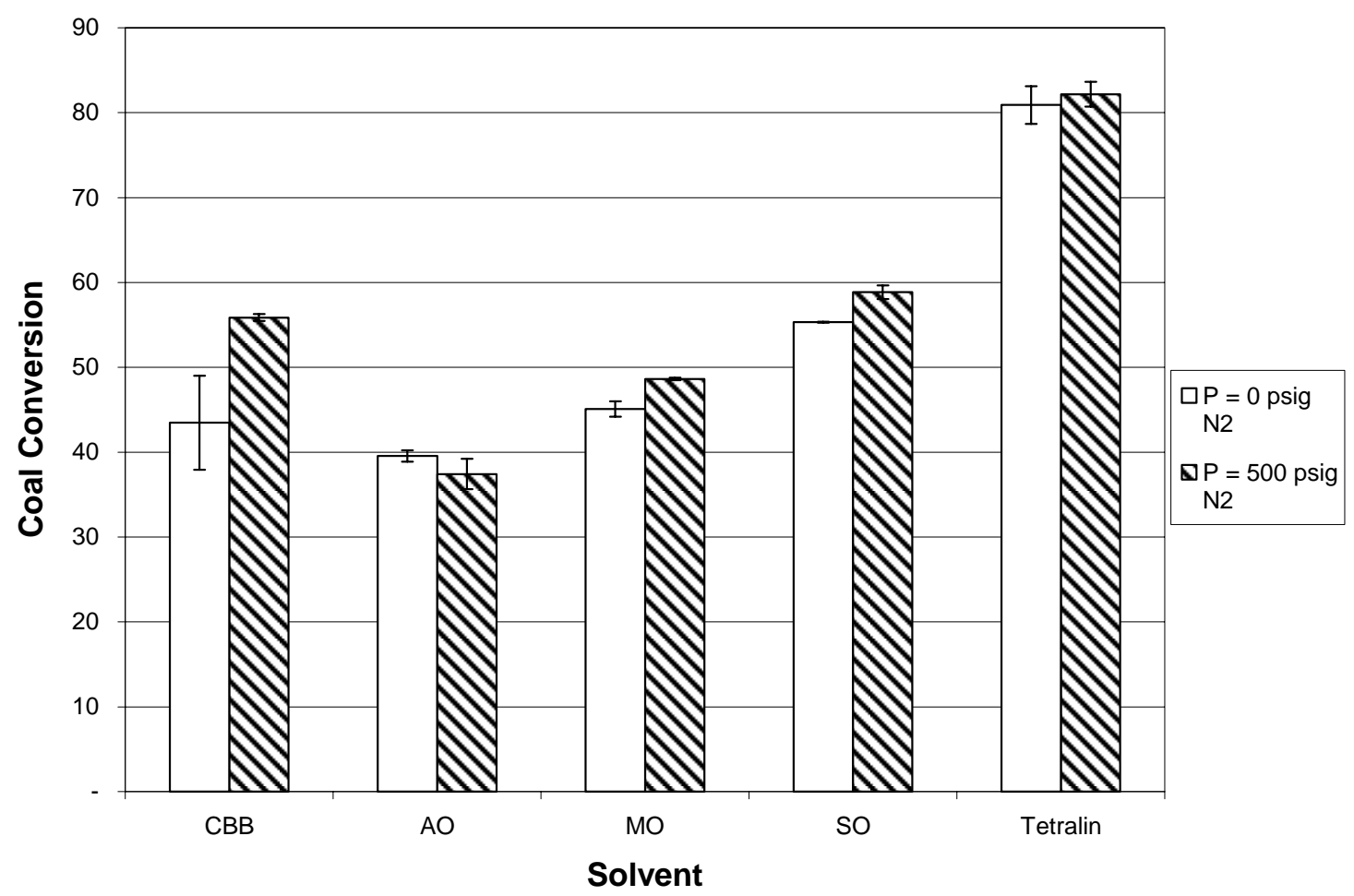

Figure 4.21 - Coal Conversion for Raw Solvents $\left(T=400^{\circ} \mathrm{C}\right)$

It can also be observed that the "level 3 " hydrogenation $\left(375^{\circ} \mathrm{C}\right.$ with $750 \mathrm{psig} \mathrm{H}_{2}$, cold - HCBB-L3) of the CBB performs the best over the other creosote oil variants (Figure 4.22). In fact, this solvent works almost as well as tetralin, with tetralin converting only about ten percent more of the coal. It can, however, be seen that the difference between the "level 2" and "level 3" hydrogenations of the CBB is small and they digest the coal to within a close range to each other. This would imply that it would not be necessary to hydrogenate the solvent at the "level 3" conditions. However, for the purpose of this study, the "level 3" hydrogenation was taken to be the best set of conditions. 


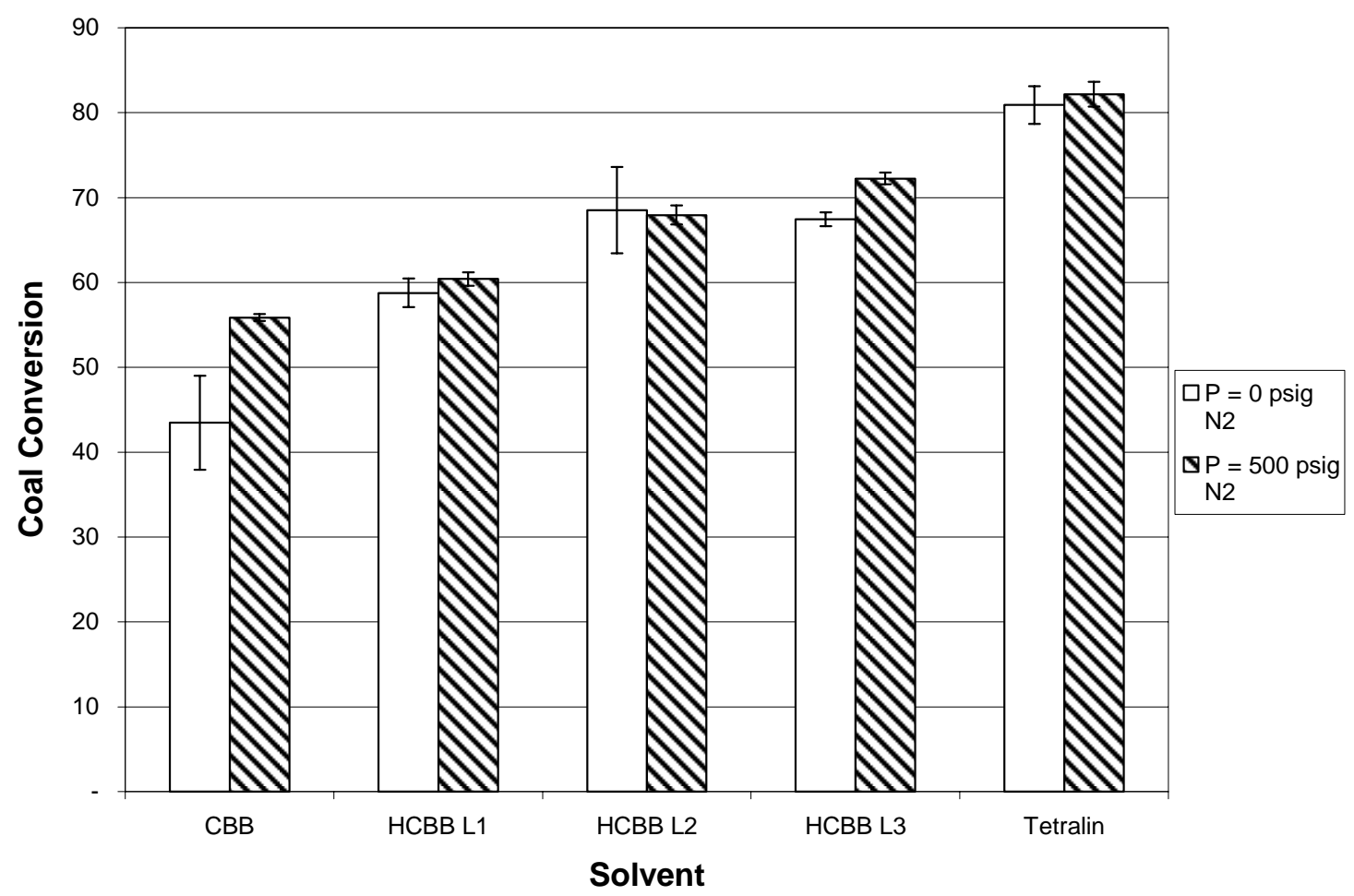

Figure 4.22 - Conversion vs. Hydrogenation Level $\left(T=400^{\circ} \mathrm{C}\right)$

It can be seen from Figure 4.23 that using combinations of solvents can often result in better conversion than the pure solvents alone. Specifically, the use of HCBB in combination with slurry oil (SO) or hydrogenated slurry oil (HSO) gives better results than the slurry oil alone. This is likely due to the better dispersive effects provided by the coal derived solvent. The runs made with slurry oil alone were often observed to be clumpy and even chunky during extraction from the reactor. This effect was not observed when the solvents were used in combination. It is believed that while the slurry oils might have good hydrogen donor capabilities, they do not have good solvation properties (i.e. it does not keep the coal or coal fragments in solution). 


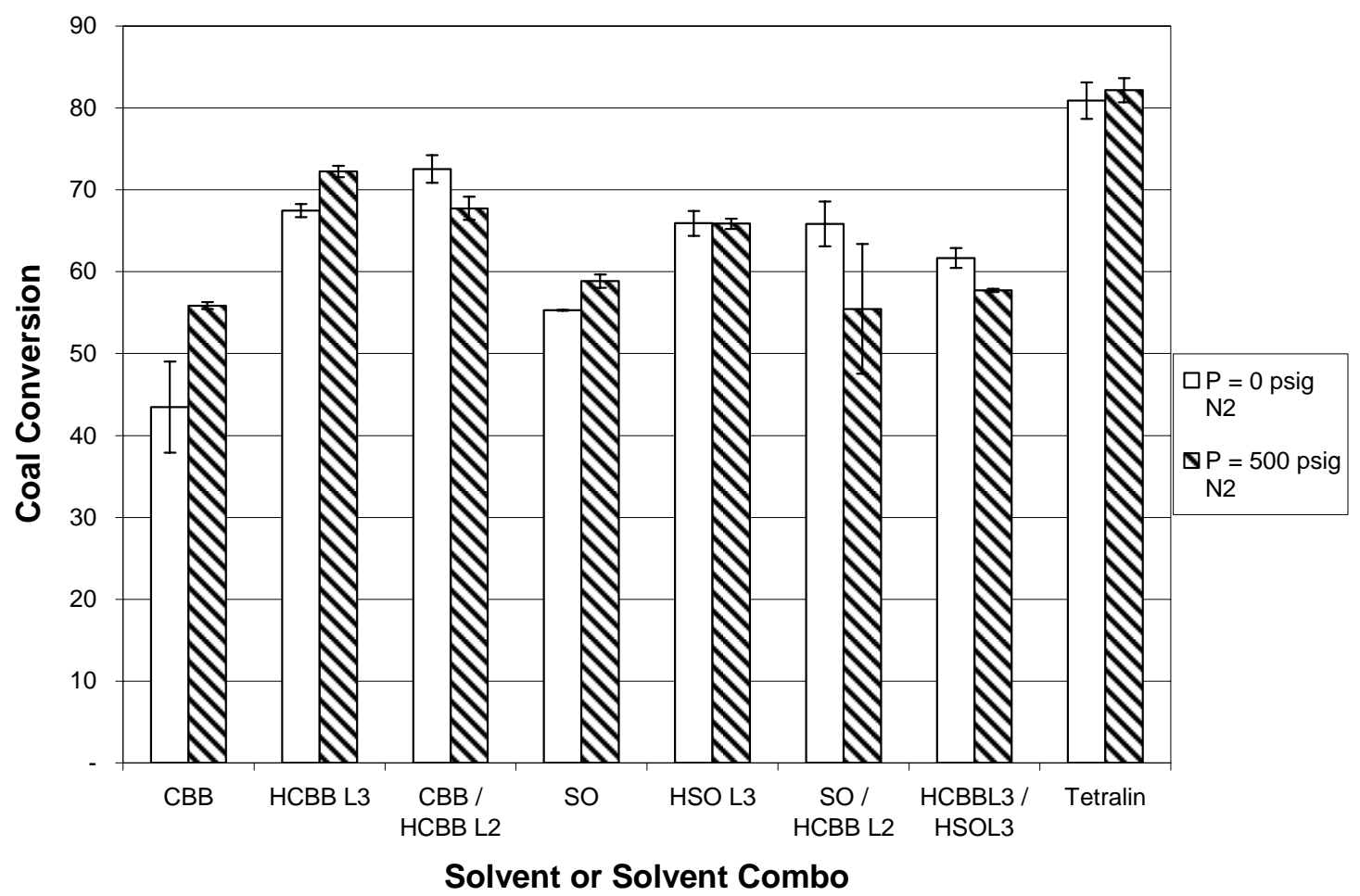

Figure 4.23 - Coal Conversion vs. Solvent Choice $\left(T=400^{\circ} \mathrm{C}\right)$

The final determination made from the tubing bomb reaction data is the fact that nitrogen pressure appears to have no appreciable effect. As can be seen from each of the figures of data above, the difference between the pressurized and un-pressurized runs is typically insignificant, and it is not consistently better with a nitrogen blanket or pressurized with nitrogen up to 500 psig.

\subsection{One Gallon Scale-Up Studies}

After the parametric studies run in the tubing bomb mini-reactors were complete, the best three solvents were chosen for scale-up work. It was decided to also use the Maraflex oil (after hydrogenating it at Level 3) in the scaled-up tests, as this oil was 
much lighter than the slurry oil, and was comparable with the slurry oil in coal conversion without the "chunkiness" problems associated with processing the products. The description of the six (6) scale-up liquefactions is given in Table 4.3.

Table 4.3 - 1-Gallon Scale-Up Digestion Details

\begin{tabular}{|r|c|c|c|c|c|c|}
\hline Name & A086 & A090 & A095 & A098 & A100 & B003 \\
\hline Solvent Used & HCBB-L3 & $\begin{array}{c}50 \% \text { HCBB-L3 } \\
\& 50 \% \text { HSO-L3 }\end{array}$ & HSO-L3 & HCBB-L3 & HMO-L3 & $\begin{array}{c}50 \% \text { HCBB-L3 \& } \\
50 \% \text { HMO-L3 } \\
\text { Temp. }\left({ }^{\circ} \mathrm{C}\right)\end{array}$ \\
400 & 425 & 425 & 425 & 425 & 425 \\
\hline
\end{tabular}

The first scale-up digestion, operated at $400^{\circ} \mathrm{C}$, resulted in a slightly rubbery material after air-blowing. This was believed to be a due to the coal fragments not being broken down enough. It was decided for all subsequent digestions that the temperature would be increased to $425^{\circ} \mathrm{C}$, to more thoroughly fracture the coal components and more thoroughly digest the coal. This rubberiness was greatly reduced in the second digestion using HCBB-L3 alone (A098). When examining the data from the scale-up experiments, it would appear that the larger reaction volume and more complete mixing causes an increase in conversion for the HCBB cases. The conversion here (A086 \& A098) is even better than typically obtained using tetralin (about 94\% using CBB vs. about $80 \%$ using tetralin in the tubing bombs). However, the slurry oil only case (A095) actually results in a lower coal conversion than was observed in the tubing bombs (see Figure 4.24). As with the tubing bomb studies, it is believed that this lower conversion is a result of the inability of slurry oil to keep the coal particles and fragments solubilized, thus resulting in the formation of solid chunks that resist conversion. The product liquid obtained from 
this scale-up was extremely difficult to process. The solids still present after stirring at $100^{\circ} \mathrm{C}$ for an additional few hours (an attempt to dissolve more of the material) prevented removal of the products via vacuum line. Eventually, as much material as possible was removed, then the remaining material in the reactor was thinned out by adding some THF and this material was processed with the centrifuge residue in the THF filtration step. Even including this "unrecoverable” material, the conversion was still below 50\%, and the effect of this processing difficulty can be observed from the percent mass balance obtained on that run, which is lower than all other runs (still over 90\%). On a more positive note, though, the hydrogenated Maraflex oil seemed to perform better than the slurry oil in the scale-up reactions. It is also important to note that the products from using the hydrogenated Maraflex oil processed much better than the products with slurry oil or even HCBB alone. The hydrogenated Maraflex oil coal digest liquid had a very low viscosity at room temperature, thus was easier to remove from the reactor and process with centrifugation than the other products. Also, the hydrogenated Maraflex oil and HCBB (B003) combination processed easier than the slurry oil runs or the HCBB alone runs. Even though the conversion is lower using hydrogenated Maraflex oil only (A100), the combination of HMO-L3 and HCBB-L3 (B003) gave a comparable conversion to the best case (A086/98). 


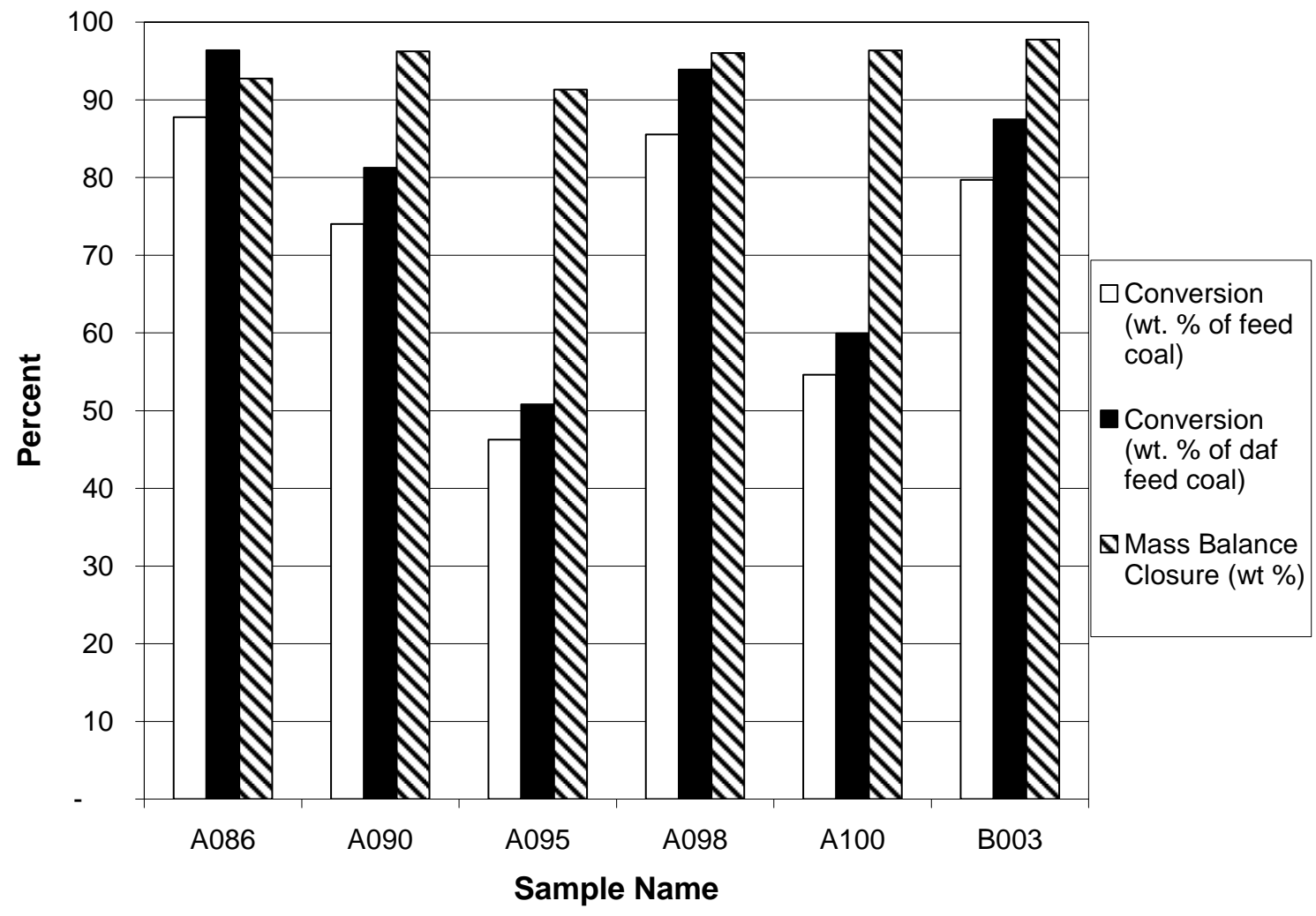

Figure 4.24 - Scale-Up Reaction Conversion vs. Solvent Choice

To determine how well the digestion has altered the starting coal material, the chemical nature of the coal liquid digests and the feed materials must be studied. The $\mathrm{C} / \mathrm{H}$ atomic ratio of the coal digests, when compared to the feed coal, decreases with the processing. The lower values are obtained, as would be expected, with the petroleum solvents, while use of the HCBB alone gives only a slightly lower $\mathrm{C} / \mathrm{H}$ ratio (Table 4.4). It is important to keep in mind the coal conversion data when considering the $\mathrm{C} / \mathrm{H}$ atomic ratio. The petroleum samples converted a much smaller amount of the coal, thus a much smaller amount of the original coal material is incorporated into the final coal liquid digest. 
Table 4.4 - Atomic C/H Ratios for Coal Liquid Digests and Feed Coal

\begin{tabular}{|c|ccccccc|}
\hline & $\begin{array}{c}\text { Kingw00d } \\
\text { Coal }\end{array}$ & A086 & A090 & A095 & A098 & A100 & B003 \\
\hline $\begin{array}{c}\text { C/H Atomic } \\
\text { Ratio }\end{array}$ & 1.304 & 1.272 & 1.121 & 0.975 & 1.283 & 1.052 & 1.179 \\
\hline
\end{tabular}

The effect of the solvent properties on the conversion and coal digest properties can be seen when the atomic $\mathrm{C} / \mathrm{H}$ ratio of the solvent used is compared with the digestion conversion (Figure 4.25). The ratios for the solvent mixtures were calculated by a weighted average of the pure solvent elemental analyses. The digestions with HCBB only (A086 and A098) give the best conversion, and yet have the highest $\mathrm{C} / \mathrm{H}$ ratio. This must be at least partially attributed to the inability of the slurry oil to properly solvate the coal, but the trend seems to indicate that a solvent with a higher $\mathrm{C} / \mathrm{H}$ ratio (one closer to that of the feed coal), or a greater aromaticity, will give the best coal conversion in this process. Beyond the total amount of hydrogen present in the solvent, the types of hydrogen present may have an effect on the conversions observed in the digestion. The results of FTIR analysis show the relative amounts of aromatic and aliphatic hydrogen present in each of the solvents, and when plotted against the conversions, it is obvious the type of hydrogen present in the solvent has an effect (Figures 4.26-29). Figure 4.26, a plot of the "aromaticity factor" versus conversion shows an increase in conversion as the aromaticity increases. This is substantiated by Figures 4.27 and 4.28 . Figure 4.27 shows that as the ratio of aromatic hydrogen to aliphatic hydrogen increases, so too does conversion. A comparison of the level of aromatic carbon-carbon bonding versus the 
total hydrogen against the conversion also indicates that a more aromatic solvent will work better in the digestion. In Figure 4.29, it becomes apparent that as the amount of aromatic hydrogen increases, or, conversely, as the amount of aromatic carbon decreases (through the partial/complete saturation of aromatic rings with hydrogen), the conversion increases. This reduction in aromatic carbon results as the rings are partially saturated with hydrogen and this hydrogen is then more labile, and is therefore more easily donated during the digestion process. So there is a point at which the addition of hydrogen ceases to break apart aliphatics and multi-ring aromatics into 2 or 3 - ring aromatics and begins to saturate the aromatics present in the solvent. The conclusion is that aromatic solvents are good digestion solvents, but hydroaromatic solvents are even better.

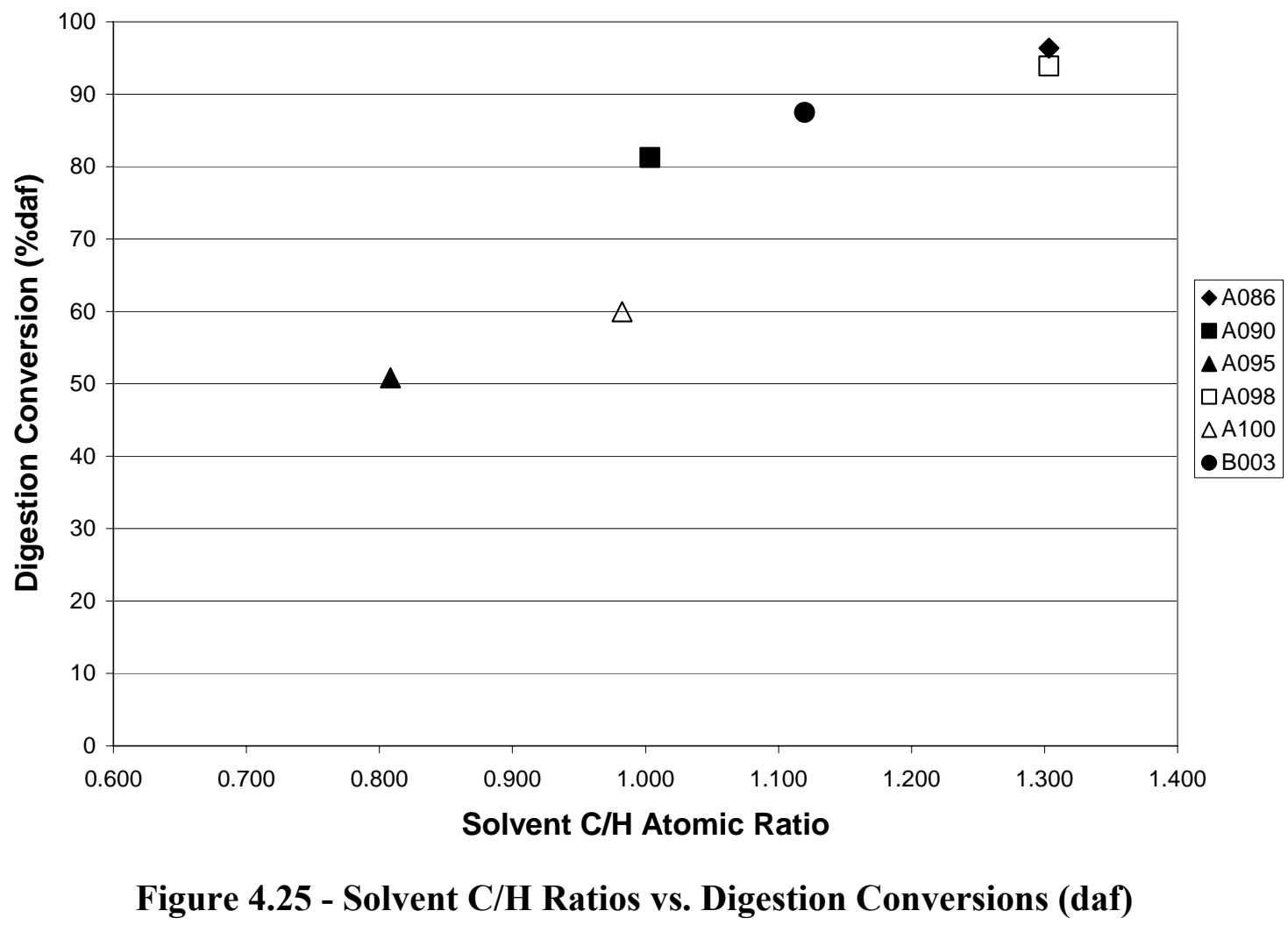




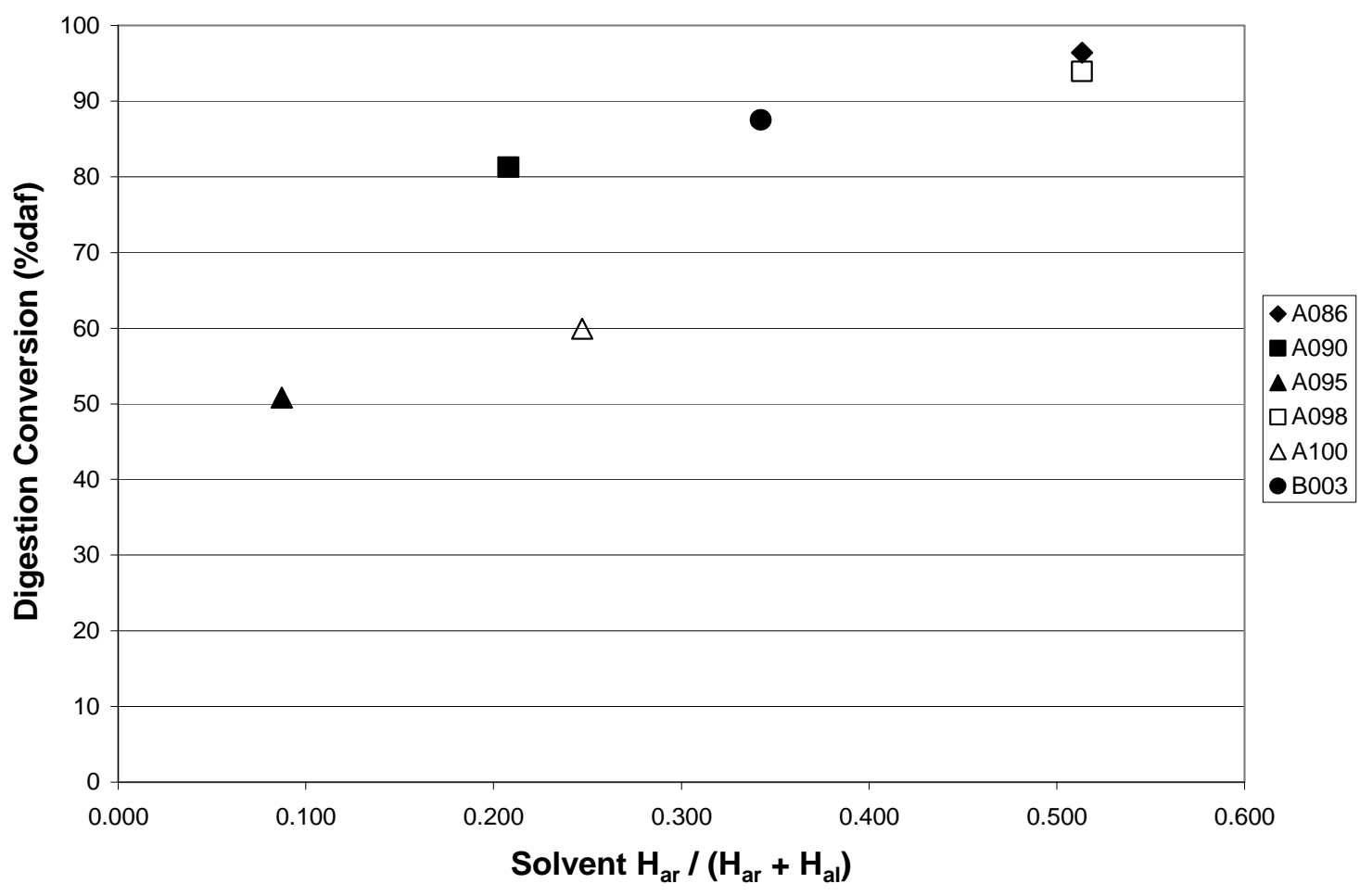

Figure 4.26 - Solvent Har / (Har + Hal) vs. Digestion Conversions (daf)

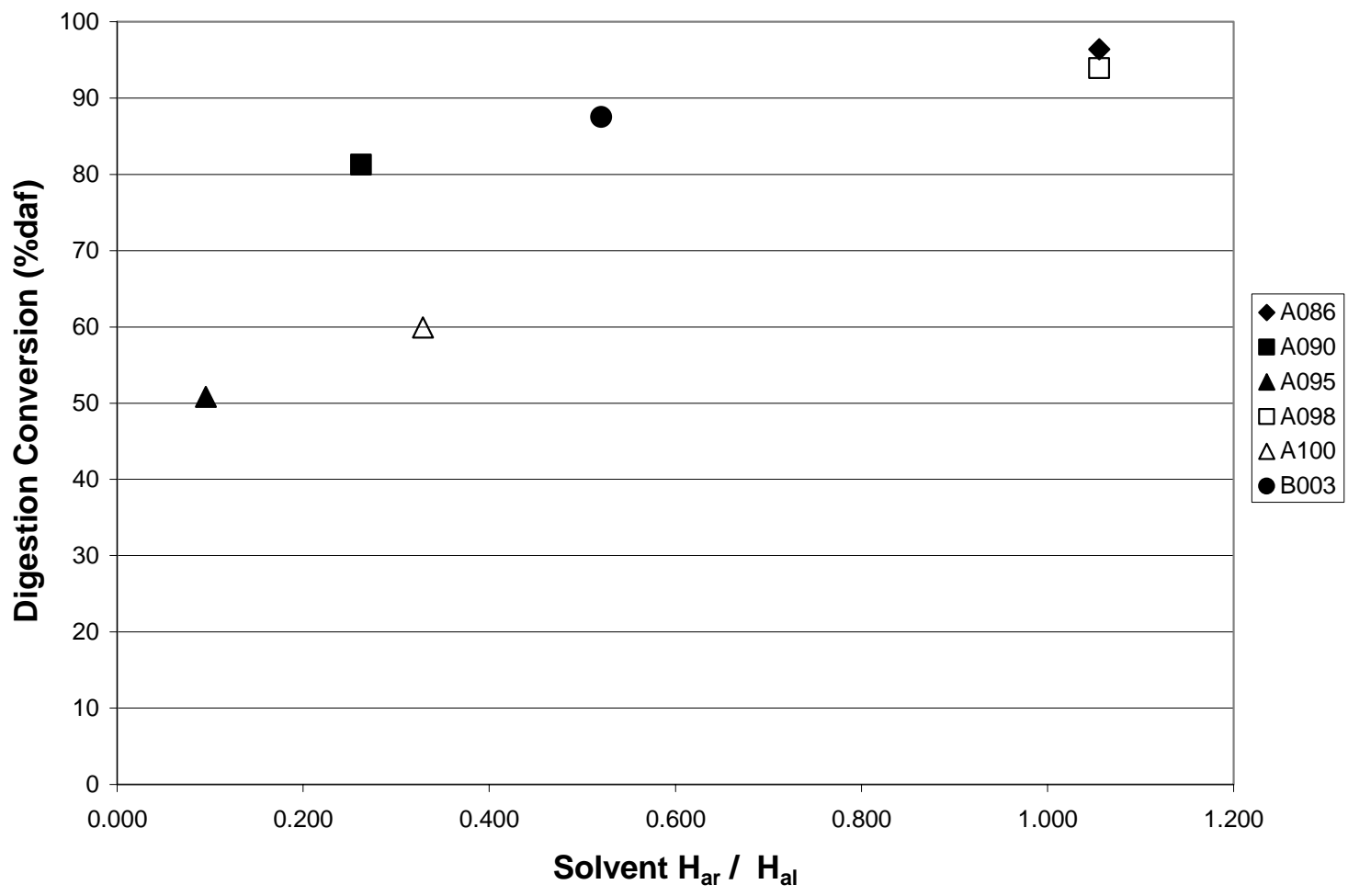

Figure 4.27 - Solvent Har / Hal Ratios vs. Digestion Conversions (daf) 


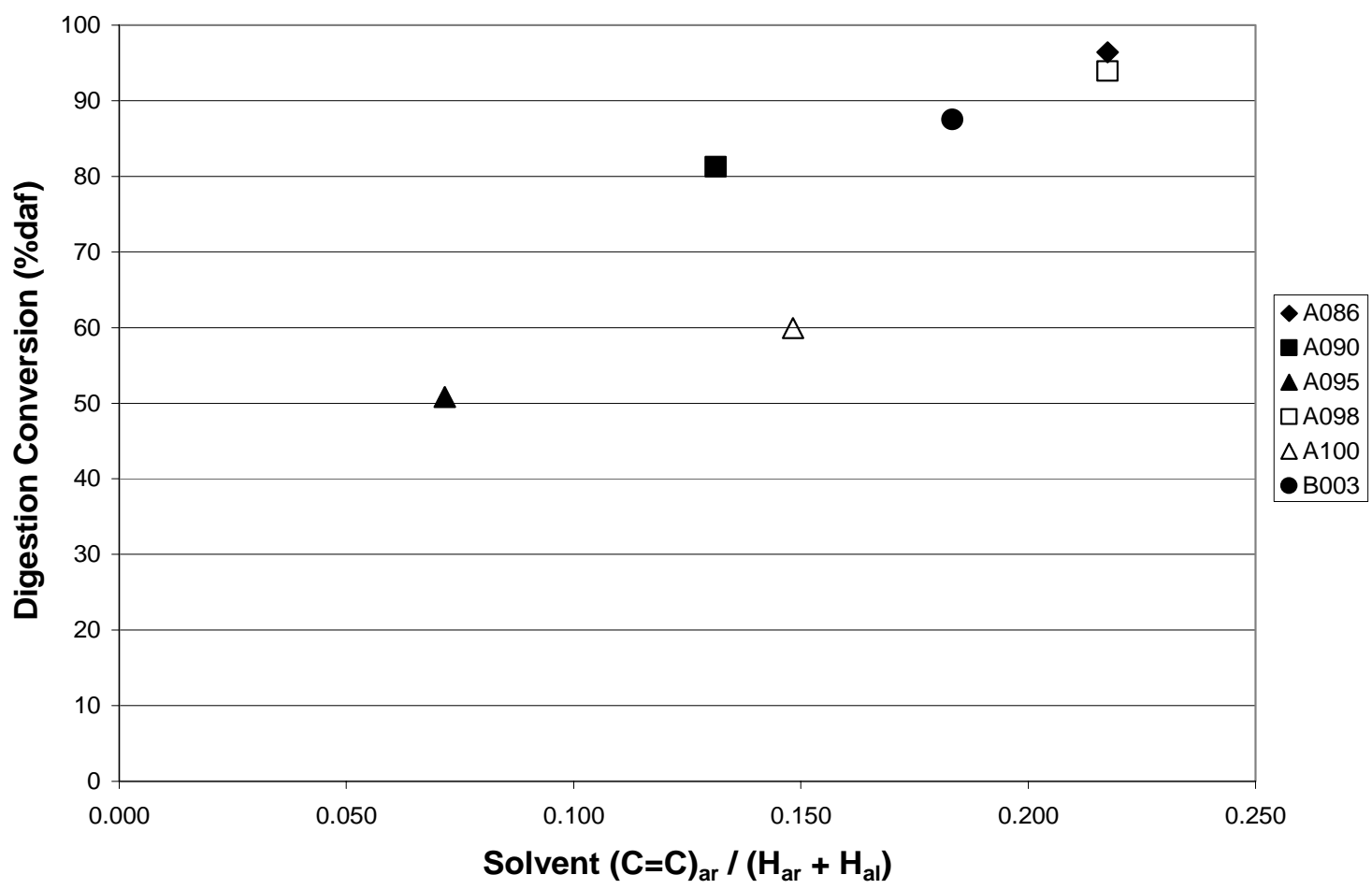

Figure 4.28 - Solvent $(\mathrm{C}=\mathrm{C})$ ar / (Har + Hal) Ratios vs. Digestion Conversions (daf)

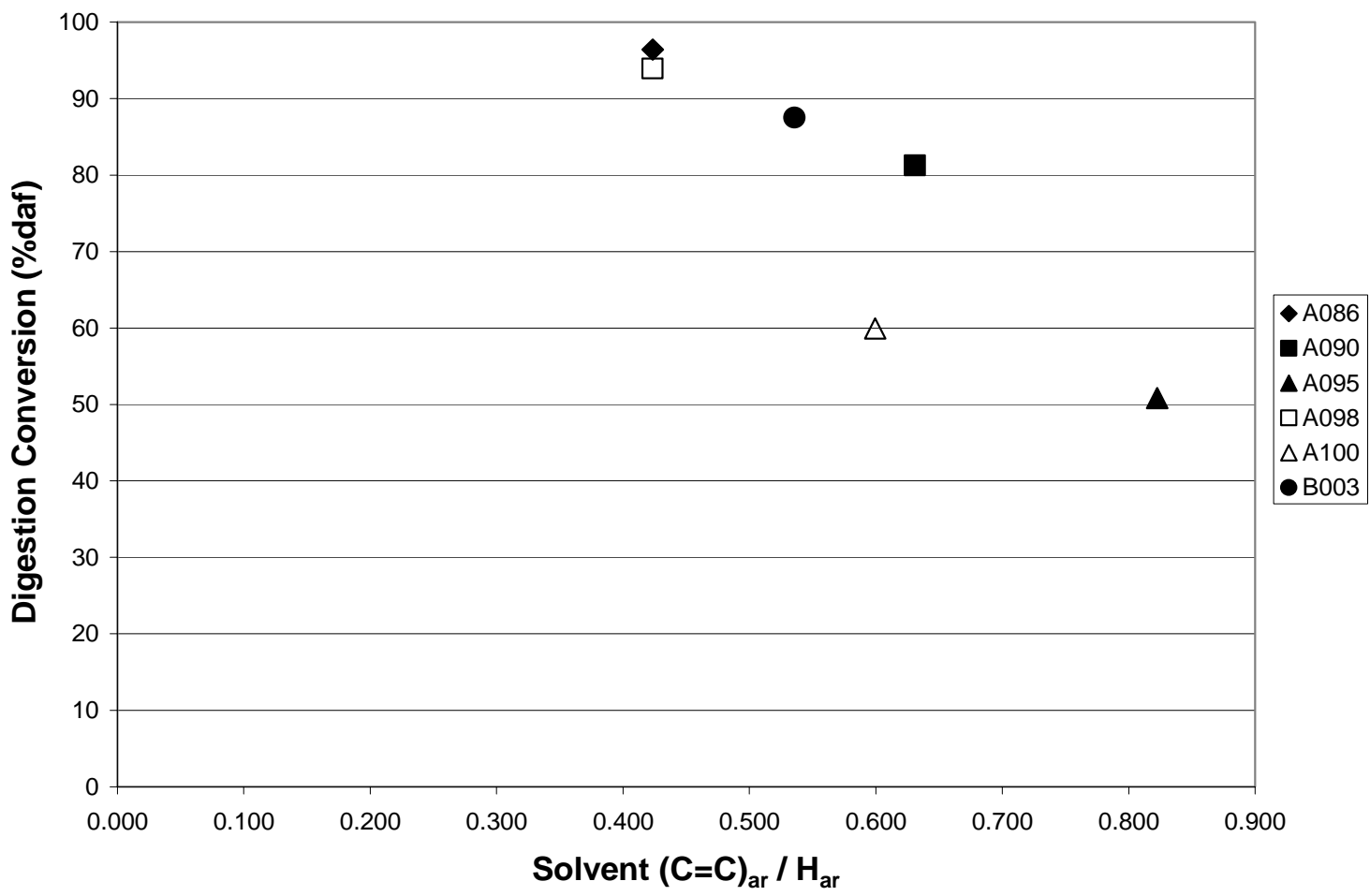

Figure 4.29 - Solvent (C=C)ar / Har Ratios vs. Digestion Conversions (daf) 


\subsection{Air Blowing Studies}

To judge the effectiveness of the entire process (solvent / coal digestion followed by air-blowing), the physical properties of the products must be examined. The softening point of the air-blown sample is a vital property in the determination of the end use for which the product is suitable. For example, binder pitches typically have softening points around $110^{\circ} \mathrm{C}$. After five hours of air blowing, the softening points of the samples produced show softening points in excess of $110^{\circ} \mathrm{C}$ (see Figure 4.30). This implies that very short air blowing times are required to increase the softening point of the coal digests to the desired level for producing binder pitch. This property is less important for producing coke feeds.

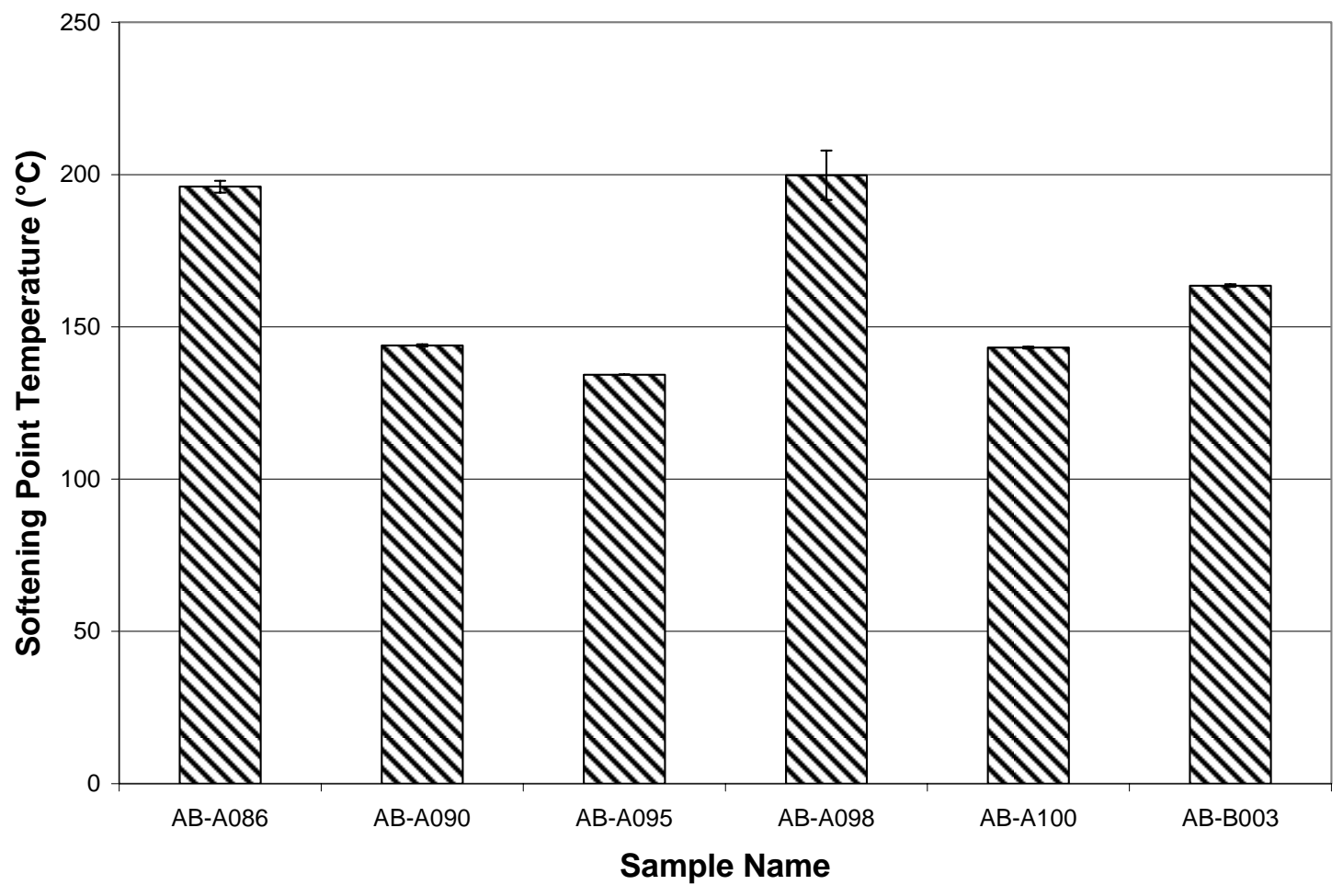

Figure 4.30 - Softening Point $\left({ }^{\circ} \mathrm{C}\right)$ for Air Blown Digests 
The ash content of the coal digests and air blown digests is also a significant parameter. High ash content will prevent the product from being used successfully in the formation of aluminum industry anodes. The mineral matter present in an anode increases the rate at which the anode is oxidized and therefore affects efficiency. An anode with high ash content will not last as long, and thus the aluminum plant will require more of the anodes more often. To decrease this need, the ash content in the coal must be reduced during the digestion to as low a level as is possible. This is because any subsequent processing the digest will undergo (air-blowing, coking, calcining) will decrease the amount of other constituents in the digest, and thereby increase the concentration of the residual mineral matter in the final anode. The ash contents of the coal liquids and air blown pitches are shown in Figure 4.31. They have all been decreased significantly from the $8.92 \%$ ash present in the feed coal. While these values are much better, they do not quite meet the stringent demands on coke feeds and binder pitches. However, this is not unexpected, as the separation step in processing the coal liquid would work best at an elevated temperature, which keeps the converted product in a much more fluid state. In this study, the centrifugation used to separate the products from the residues was done with the materials initially in excess of $100^{\circ} \mathrm{C}$. Unfortunately, the centrifuge used in this study is incapable of running at elevated temperatures, and the material in the bottles began to cool immediately, thus increasing the viscosity of the coal digest liquids, and hindering the separation of the residue. In a production scale facility, this problem could be eliminated using high temperature liquidsolid separation techniques. 


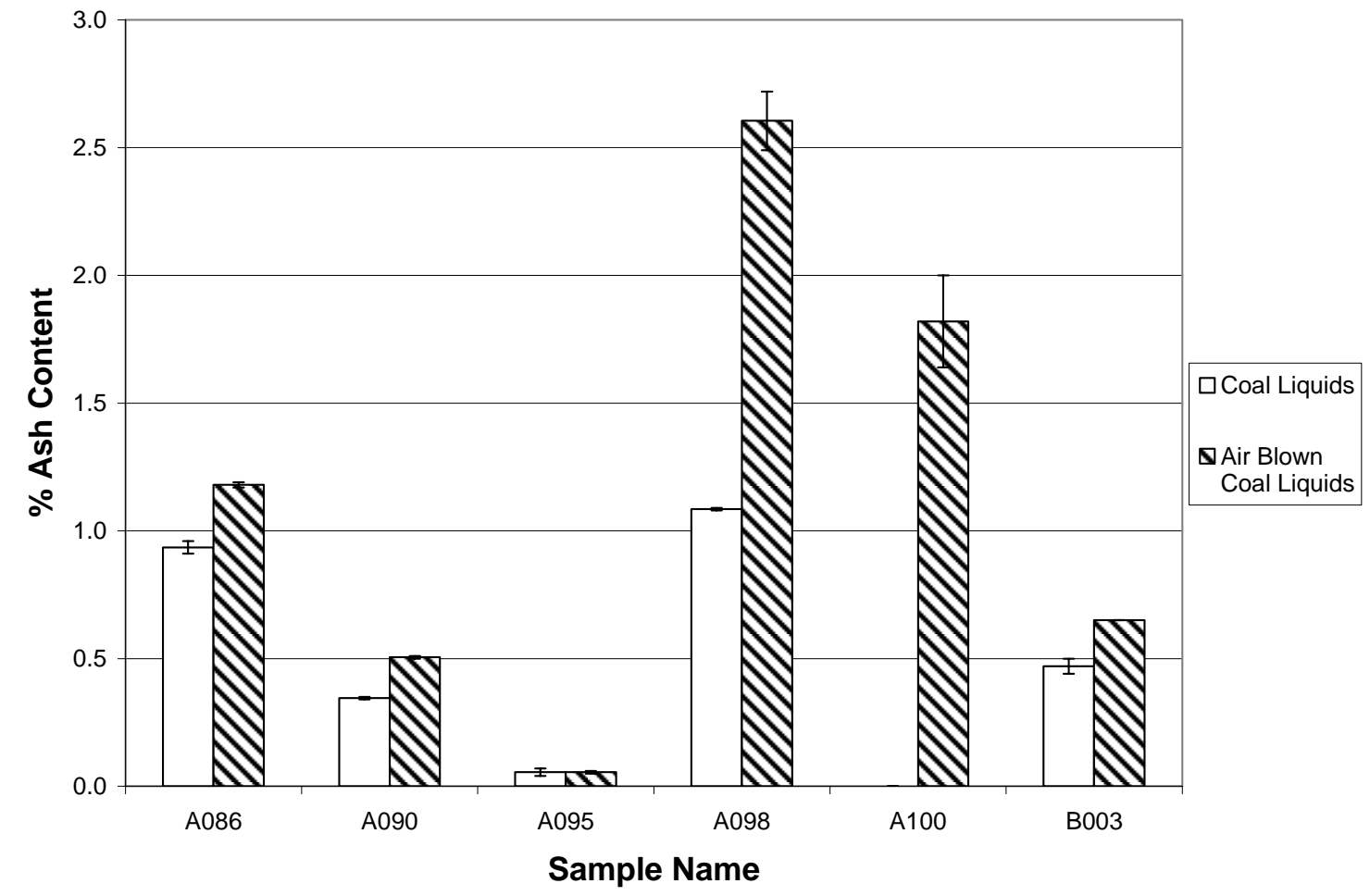

Figure 4.31 - Ash Content in Coal Digest Liquids and Air Blown Digests

The coke yields of these products are also essential data. As can be seen in Figures 4.32 and 4.33, the coke yields of the air-blown digests are greater than that of the liquid digests. This supports the use of air-blowing as a method to alter pitches to fit end uses. It must be noted, though, that the coke yields of the air blown digests are not as high as would be desirable for coke feeds or binder pitches. Additional air blowing, depending on the end product desired, could increase the coke yields, but would also increase softening points, which would negate the use of these materials as binders. This may be solved either through varying the temperature and residence time of the air blowing, or perhaps through some distillation prior to air blowing to remove some of the entrained solvent. 


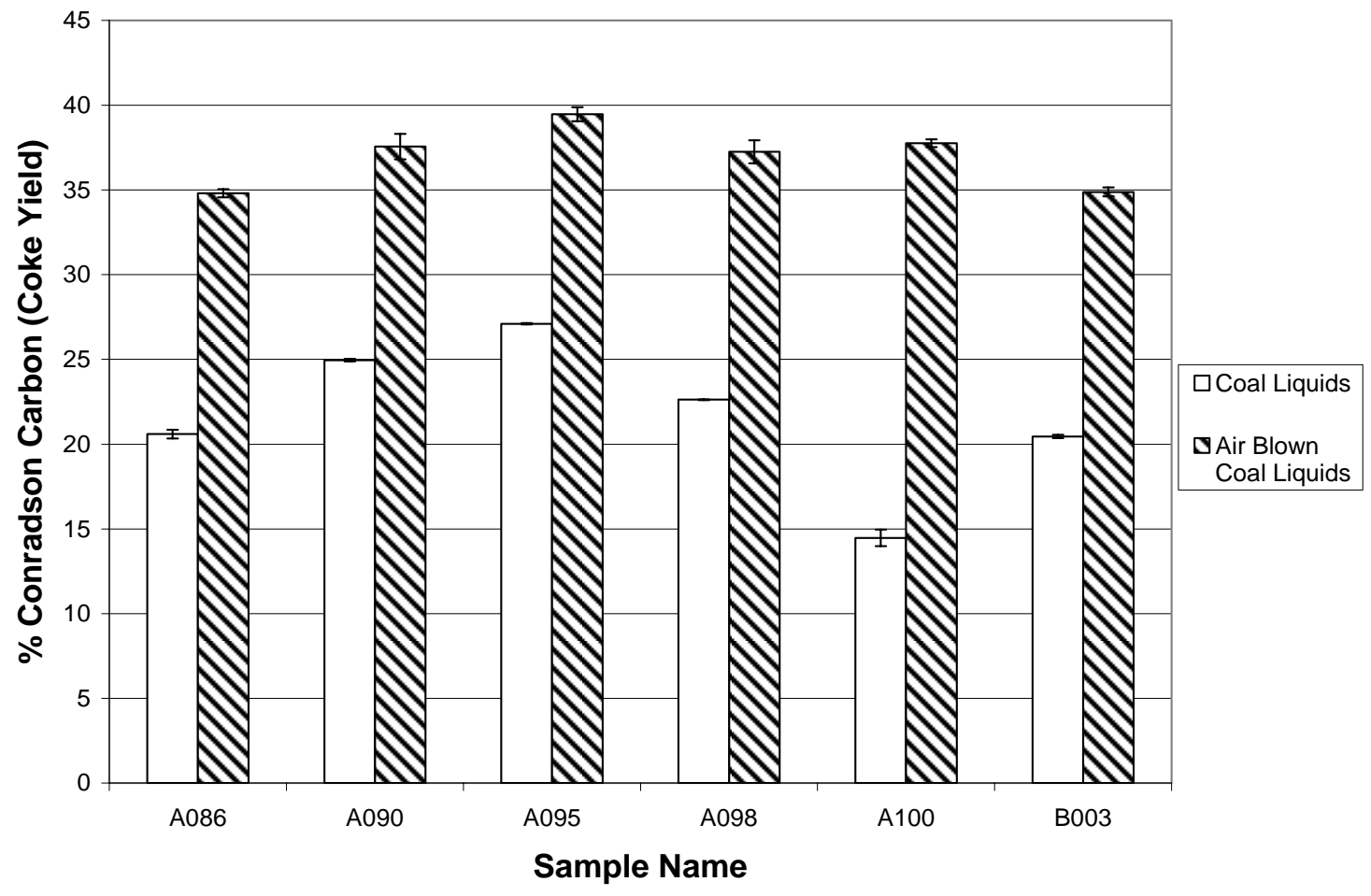

Figure 4.32 - Conradson Carbon Coke Yield of Coal Digests

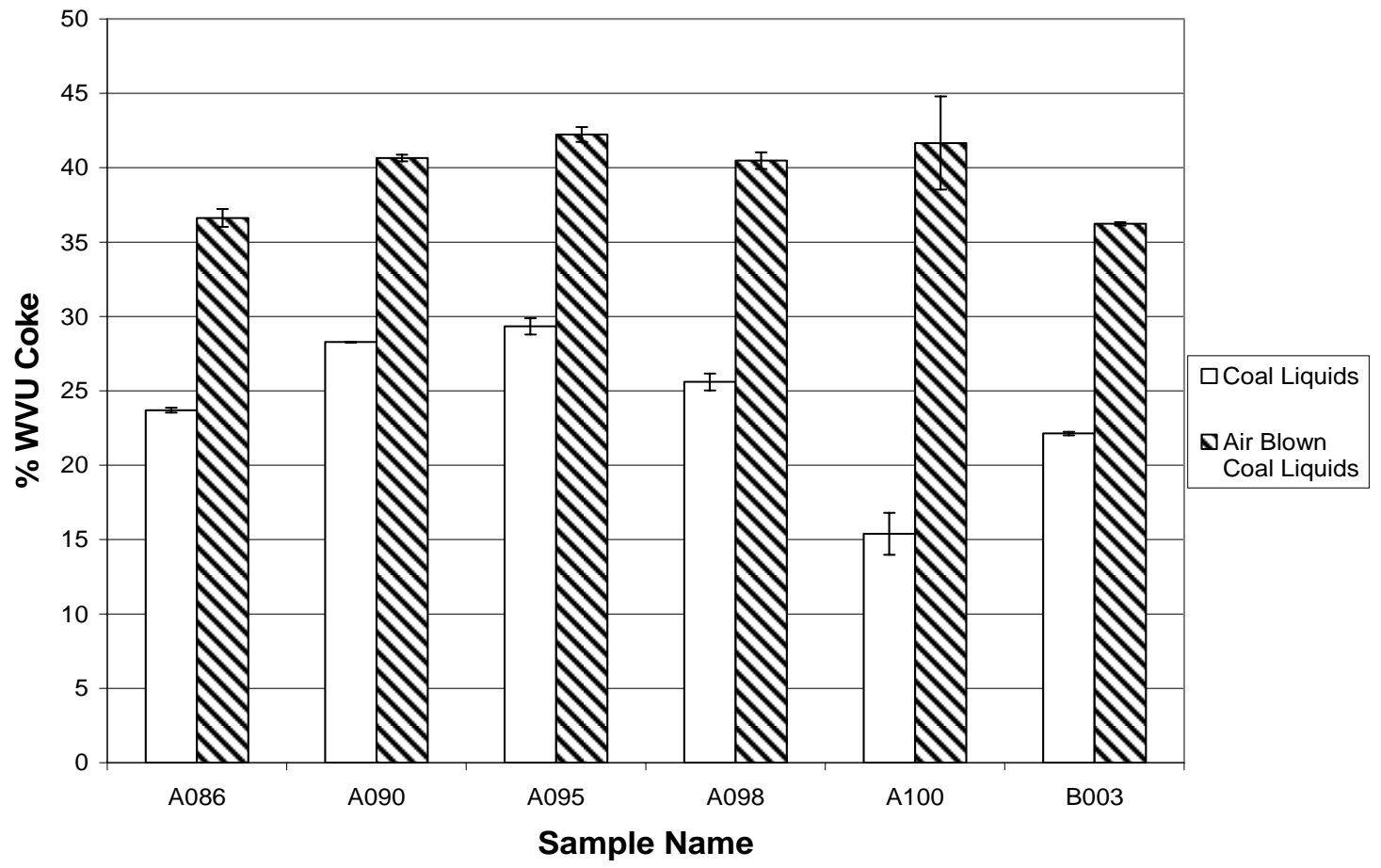

Figure 4.33 - WVU Coke Yield of Coal Digests 
The air blowing also affects the solubility of the digests. The amount of toluene insoluble material in the air-blown digests almost doubles in most cases and nearly triples in one case (Figure 4.34). This is an indication of the amount of higher molecular weight species present in the digest. This is a result of lower molecular weight species crosslinking during the air-blowing or possibly from the removal of lights which are stripped off during the air-blowing, thus concentrating the toluene insolubles. Consequently, this is also followed by a reduction in the volatile material present in the digest. This is especially good for coke feeds, which should have a higher percent of fixed carbon.

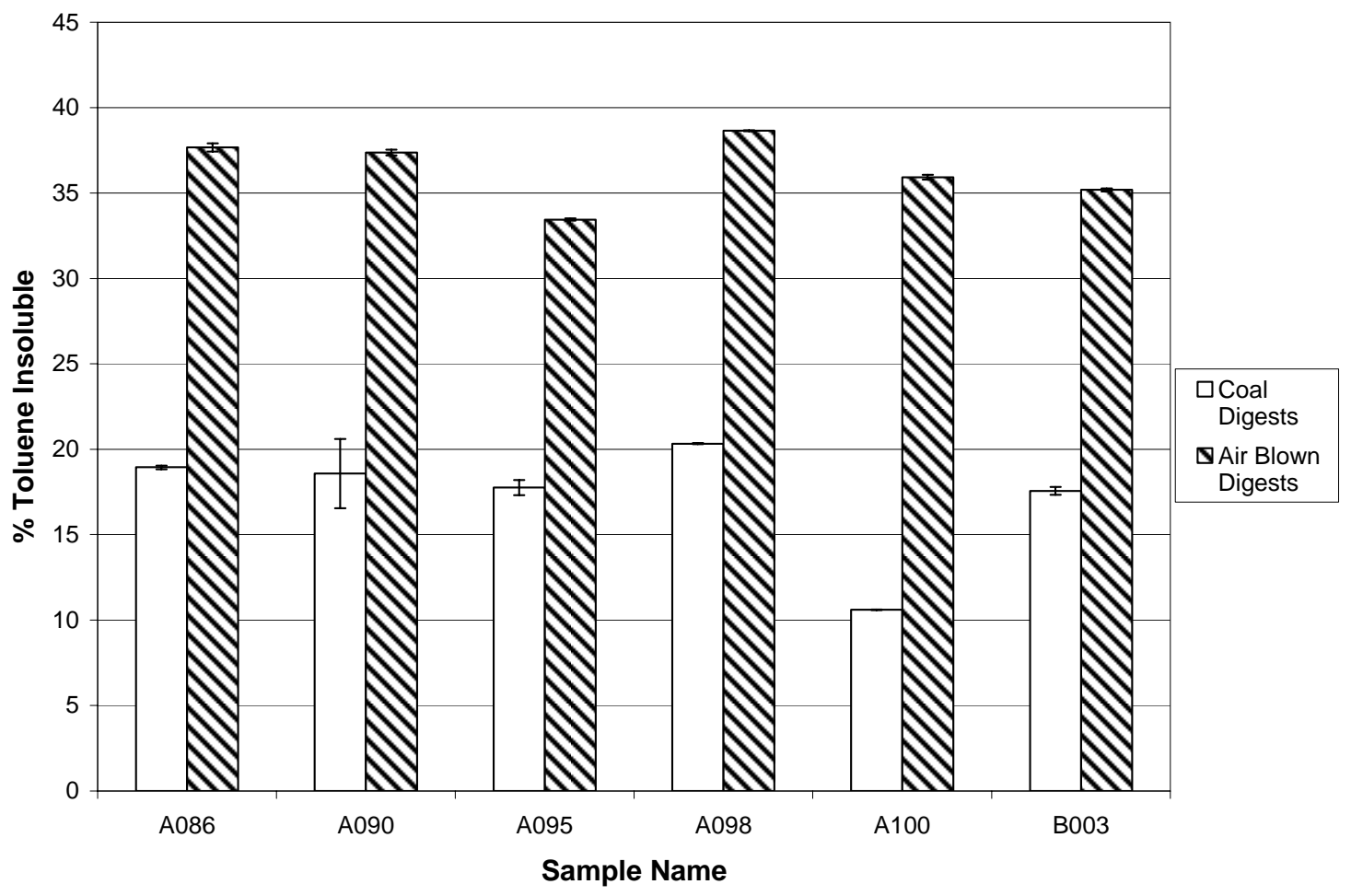

Figure 4.34 - Toluene Insolubles of Coal Digests 
The air blowing of the coal digests will also affect the density of the final product. Figure 4.35 shows the densities of the air-blown digests. It is interesting to note that the air blown A098 digest has a modestly higher density than the A086, even though they were prepared in the same method (only at a temperature of $425^{\circ} \mathrm{C}$ rather than $400^{\circ} \mathrm{C}$ ). This may imply that the more complete digestion that occurs at the higher temperature results in more lower molecular weight compounds that are more readily cross-linked during air-blowing.

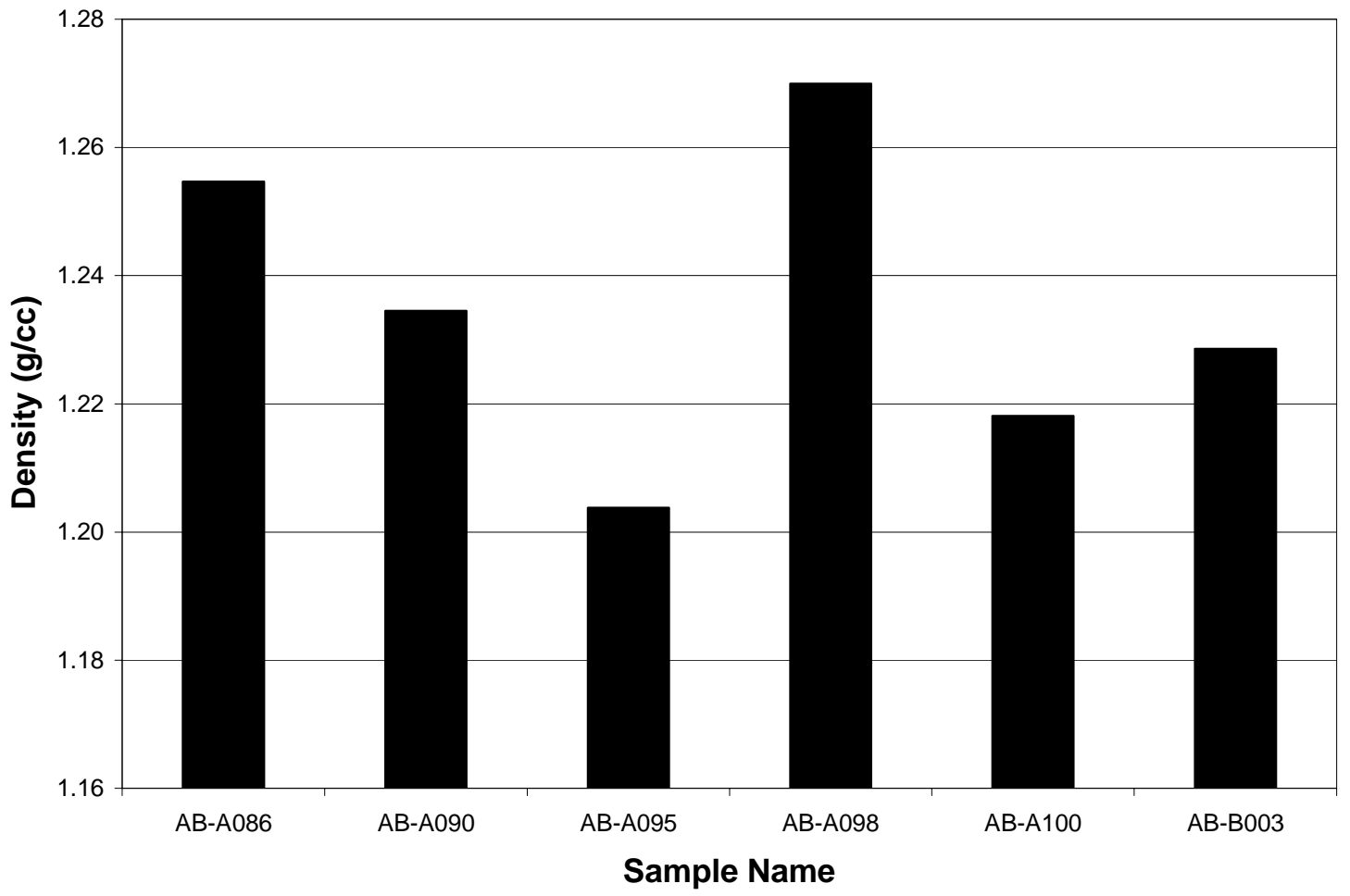

Figure 4.35 - Air Blown Sample Densities

The viscosity of the coal digests is much lower than the viscosities of the airblown coal digests. Each digest is affected in a very similar manner, as the viscosity curve shifts to the right (higher temperatures) after air blowing. The important point to make from these figures is that the viscosity of the coal digests, after centrifugation, is in 
an easily manageable range $(100-1000 \mathrm{cP})$ at temperatures below $100^{\circ} \mathrm{C}$ (Figure 4.36$)$. This has positive implications on the potential large-scale production of these materials. The pumping of these materials will not have to be done at very high temperatures, even though some of them are pasty at room temperature. The lone exception is the A095 coal digest, which was produced with level-3 hydrogenated slurry oil only. This coal digest liquid has a viscosity under $1000 \mathrm{cP}$ only after heated above $120^{\circ} \mathrm{C}$. 
A)

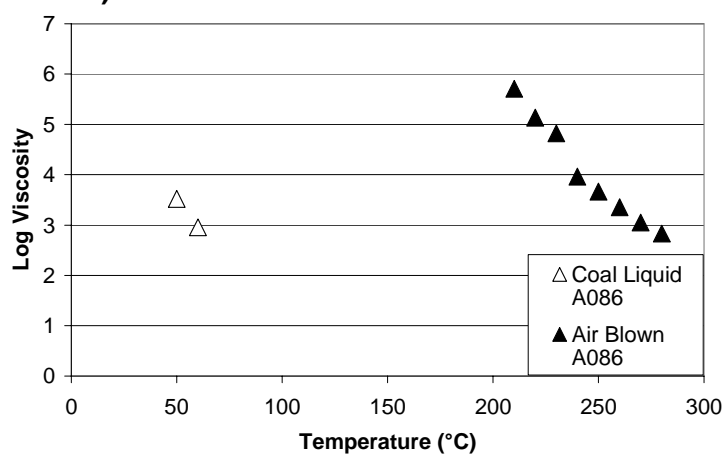

C)

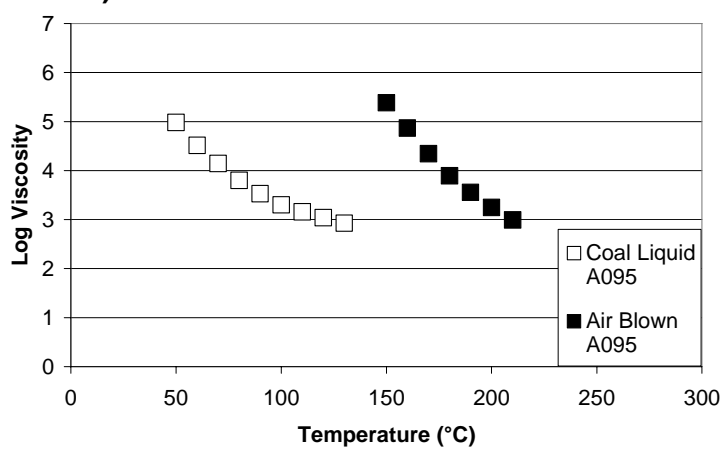

E)

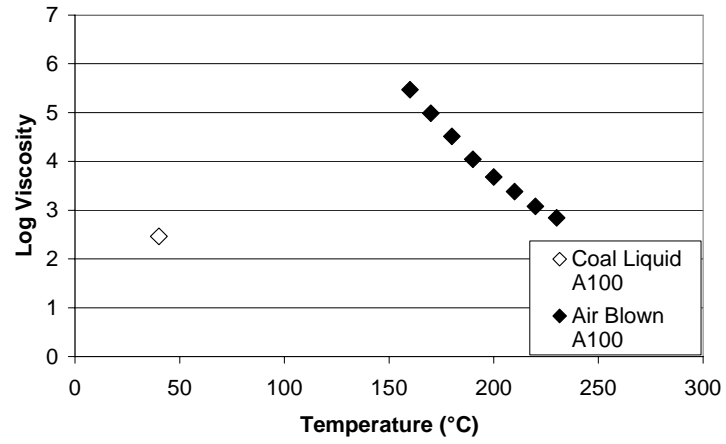

B)

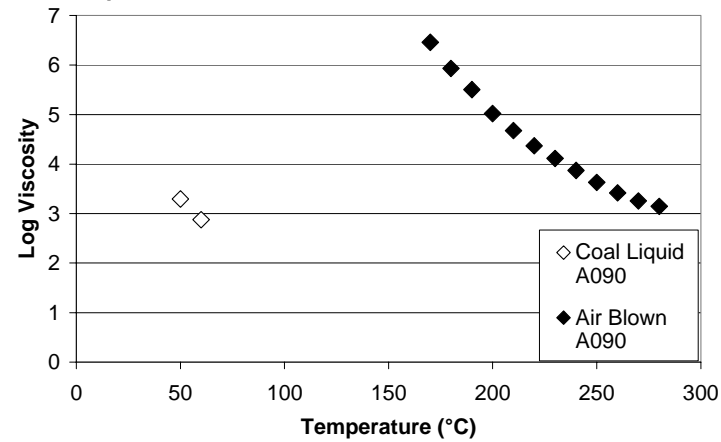

D)

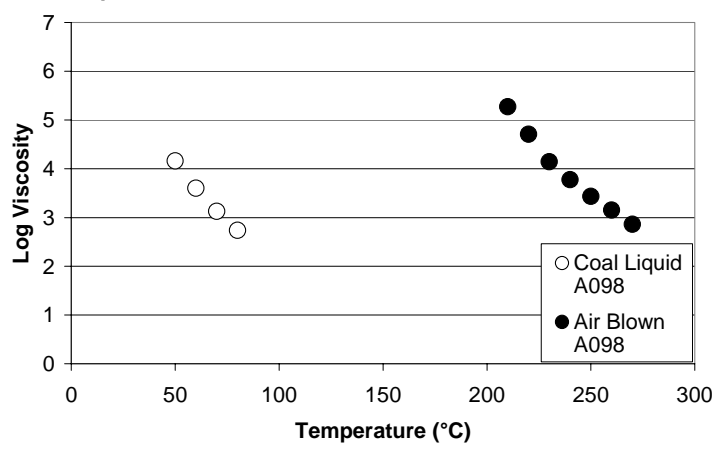

F)

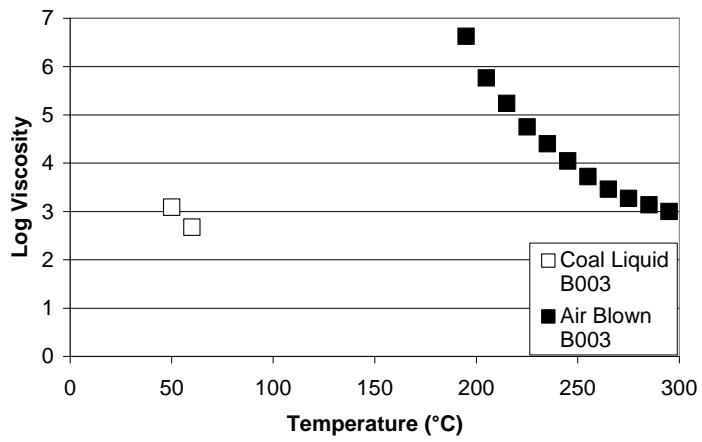

Figure 4.36 - Viscosity Vs Temperature for Coal Digests and Air-Blown Coal Digests: A) A086, B) A090, C) A095, D) A098, E) A100, F) B003

The cokes produced in the WVU Coke tests were also examined under the microscope to determine the microstructure. The cokes were placed in a plastic cup and then this cup is filled with epoxy and activator (mixed in a 5:1 ratio). After the epoxy hardens, the disks were next polished and cleaned, then placed under the microscope. As 
can be seen from the photomicrographs below, the carbonized coal digests give a highly anisotropic coke, but after the air-blowing, the coke produced is quite isotropic, even glassy, indicating that the air-blowing has destroyed the ability of the digest to develop anisotropic microstructure in the cokes (Figure 4.37-41).

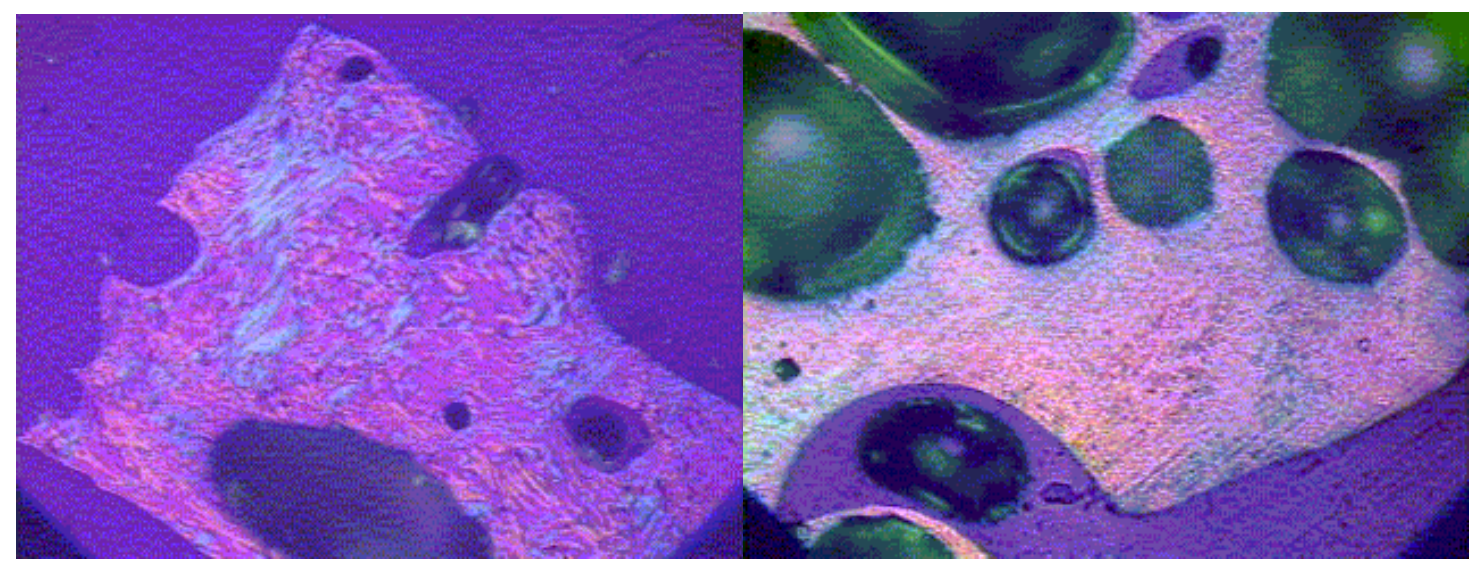

Figure 4.37 - Photomicrographs (160X) of green cokes made from A090 coal digest (left) and air-blown A090 coal digest (right)

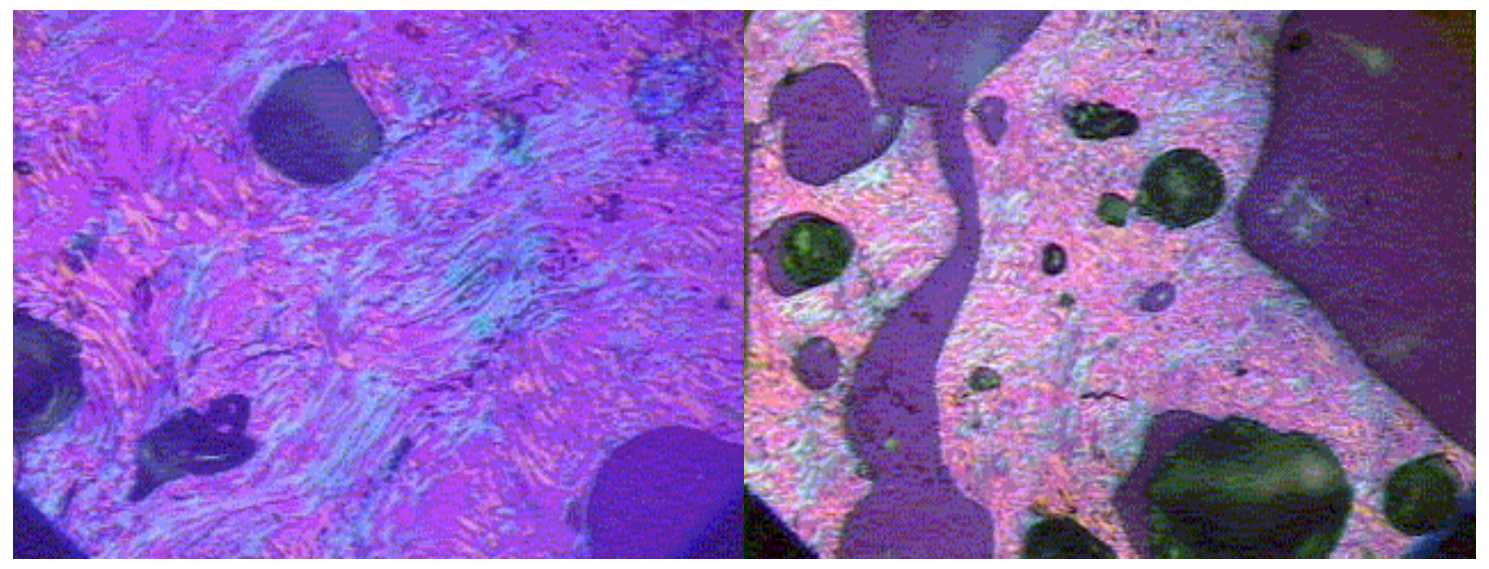

Figure 4.38 - Photomicrographs (160X) of green cokes made from A095 coal digest (left) and air-blown A095 coal digest (right) 


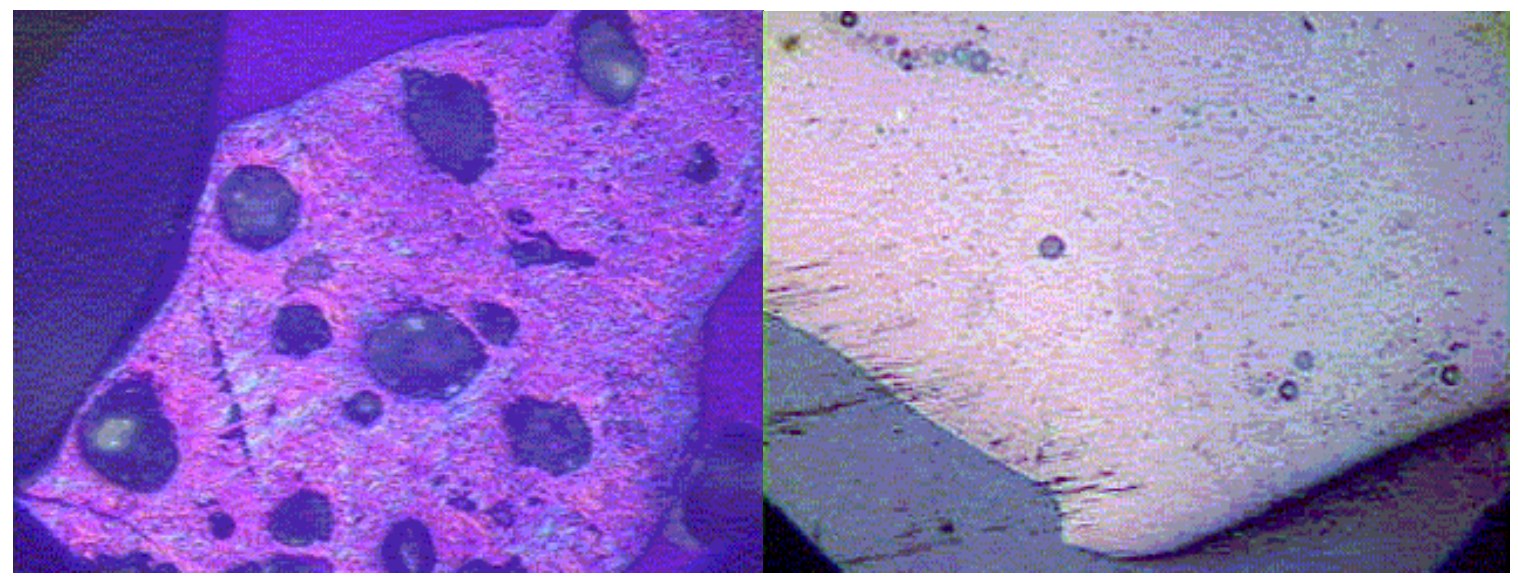

Figure 4.39 - Photomicrographs (160X) of green cokes made from A098 coal digest (left) and air-blown A098 coal digest (right)

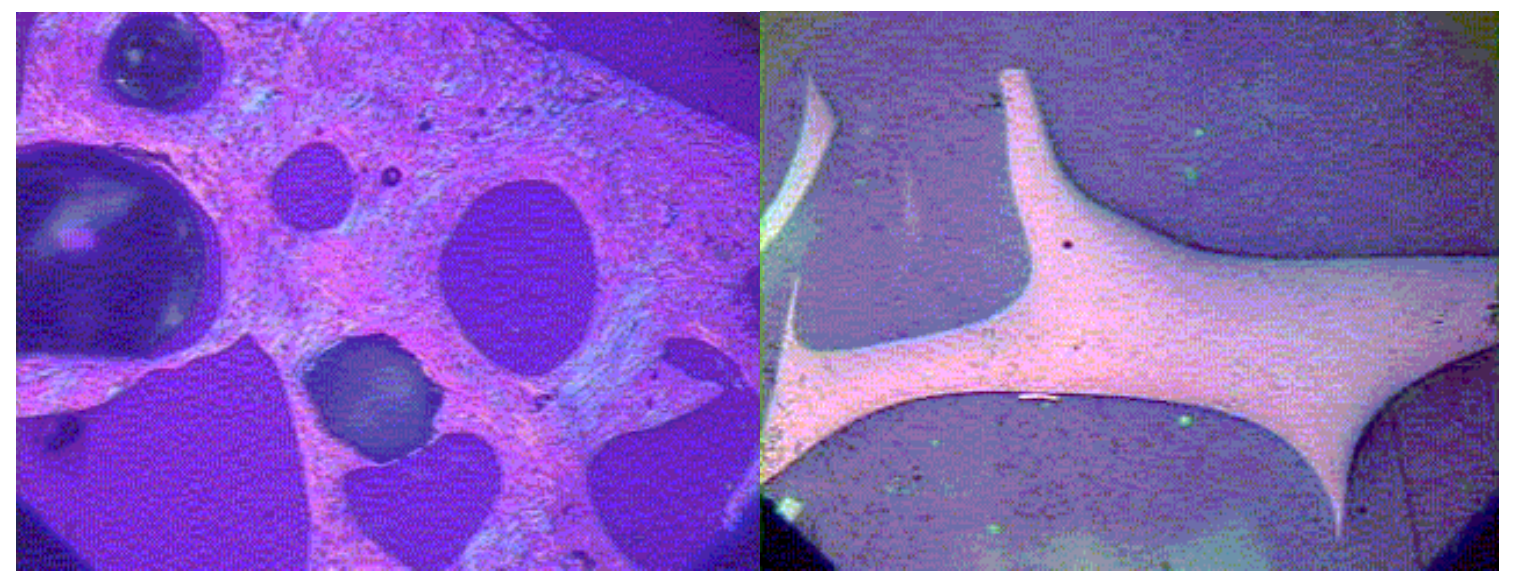

Figure 4.40 - Photomicrographs (160X) of green cokes made from A100 coal digest (left) and air-blown A100 coal digest (right)

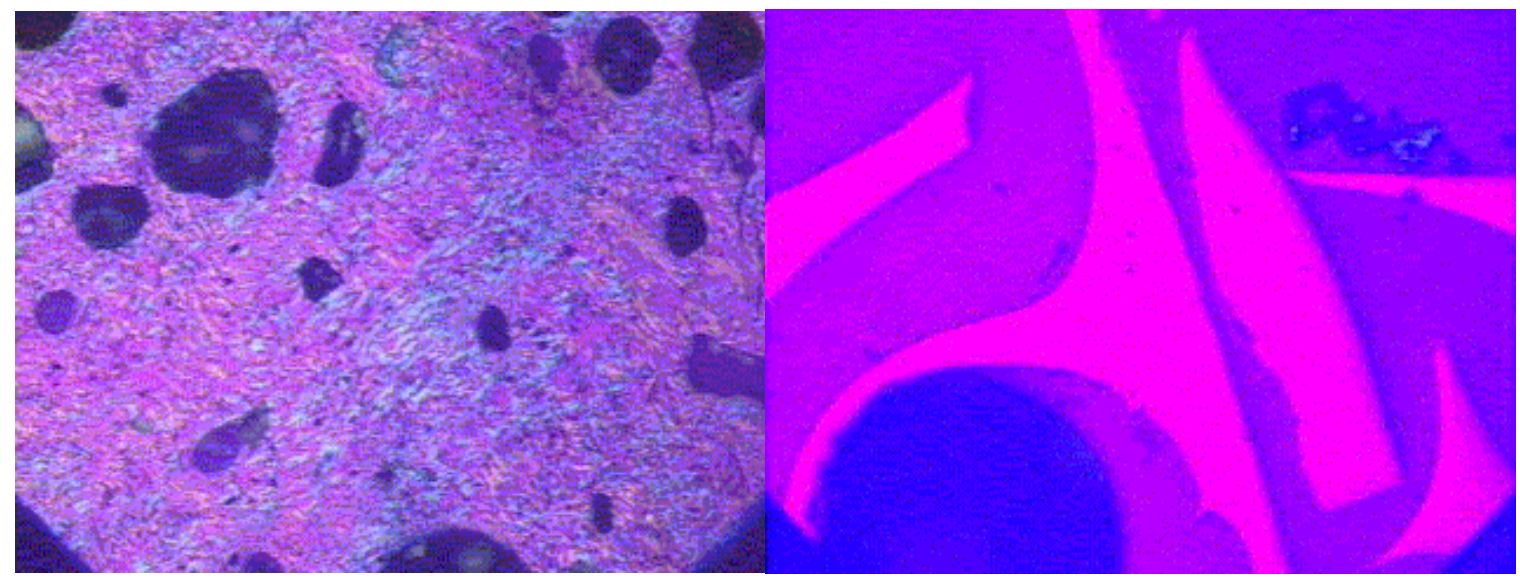

Figure 4.41 - Photomicrographs (160X) of green cokes made from B003 coal digest (left) and air-blown B003 coal digest (right) 


\subsection{Atomic Analyses}

As these products are intended for use as coke feeds for the carbon anode industry, it would be beneficial to observe how sulfur moves through this process. Sulfur in these processes not only causes the anodes to degrade more rapidly, it can also be introduced into the environment in stack gases and lead to the development of acid rain. A reduction in the sulfur content of the final product will cut down on the corrosion and environmental problems associated with using the carbon products. When compared to the original coal, the concentration of sulfur in most of the final products decreases with the digestion step (Figure 4.42). Unfortunately, though, the A095 run (hydrogenated slurry oil as solvent) shows an increase in the sulfur concentration. This is a result of the high sulfur concentration present in this solvent (Figure 4.18). Even after the air-blowing step, the sulfur concentration of the final digests is still lower than that of the original coal. When using the coal-derived solvent only (A086 and A098), there is over a 60\% reduction in the sulfur concentration. This, combined with the reduction in the ash content, makes for a more desirable pitch precursor or potential anode coke feed. 


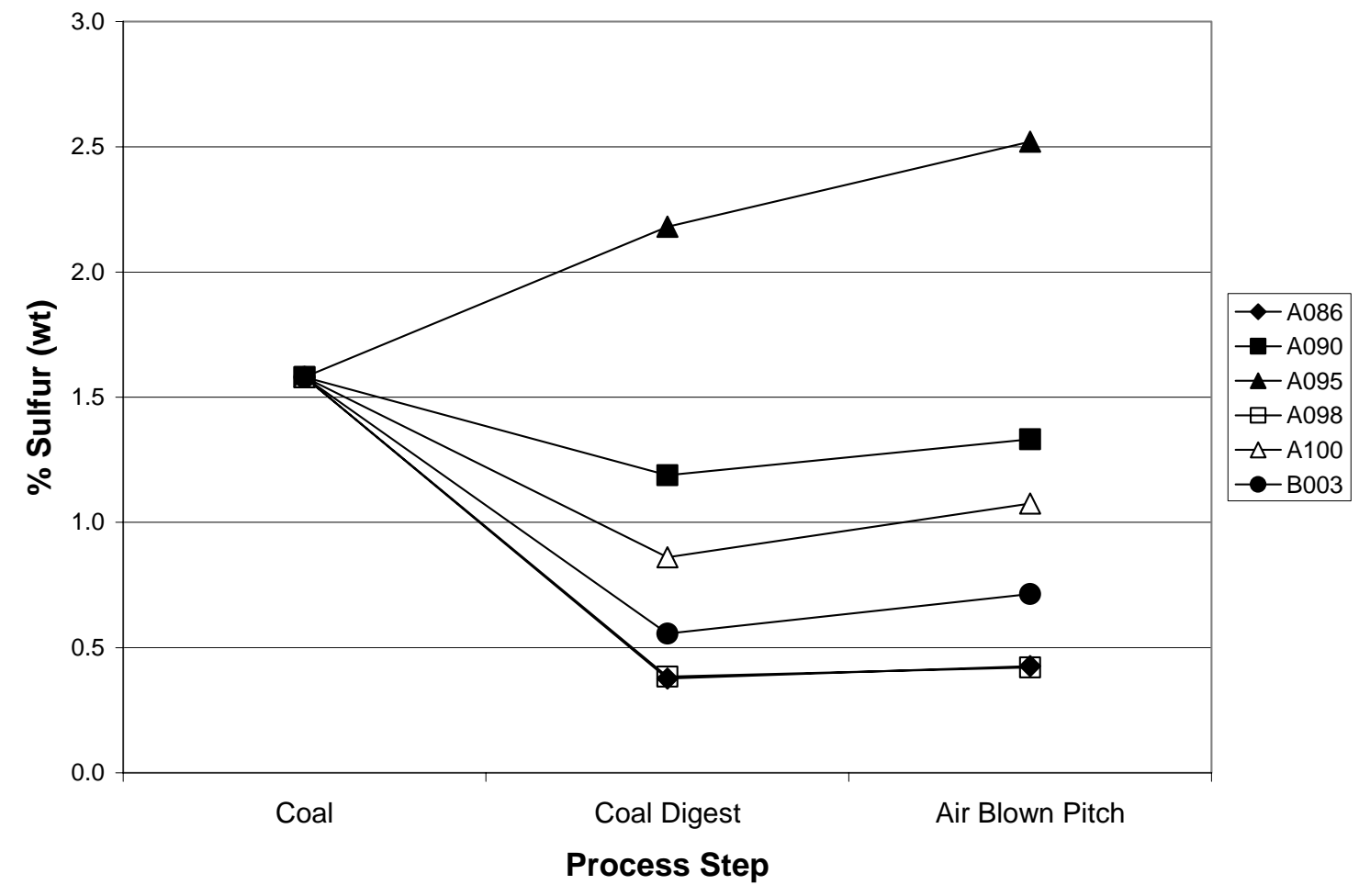

Figure 4.42 - Sulfur Concentrations in Digest Processing

While the reduction in sulfur is advantageous, there is little change in the nitrogen concentration throughout the process. The runs with HCBB-L3 only (A086 and A098) in fact show an increase in nitrogen, while the samples made with hydrogenated Maraflex oil (HMO-L3) only (A100 and B003) show a marked decrease in nitrogen concentration during the digestion step (Figure 4.43). The requirements on the nitrogen content of pitches or cokes, however, are not as stringent, so this is not necessarily a disappointing result. 


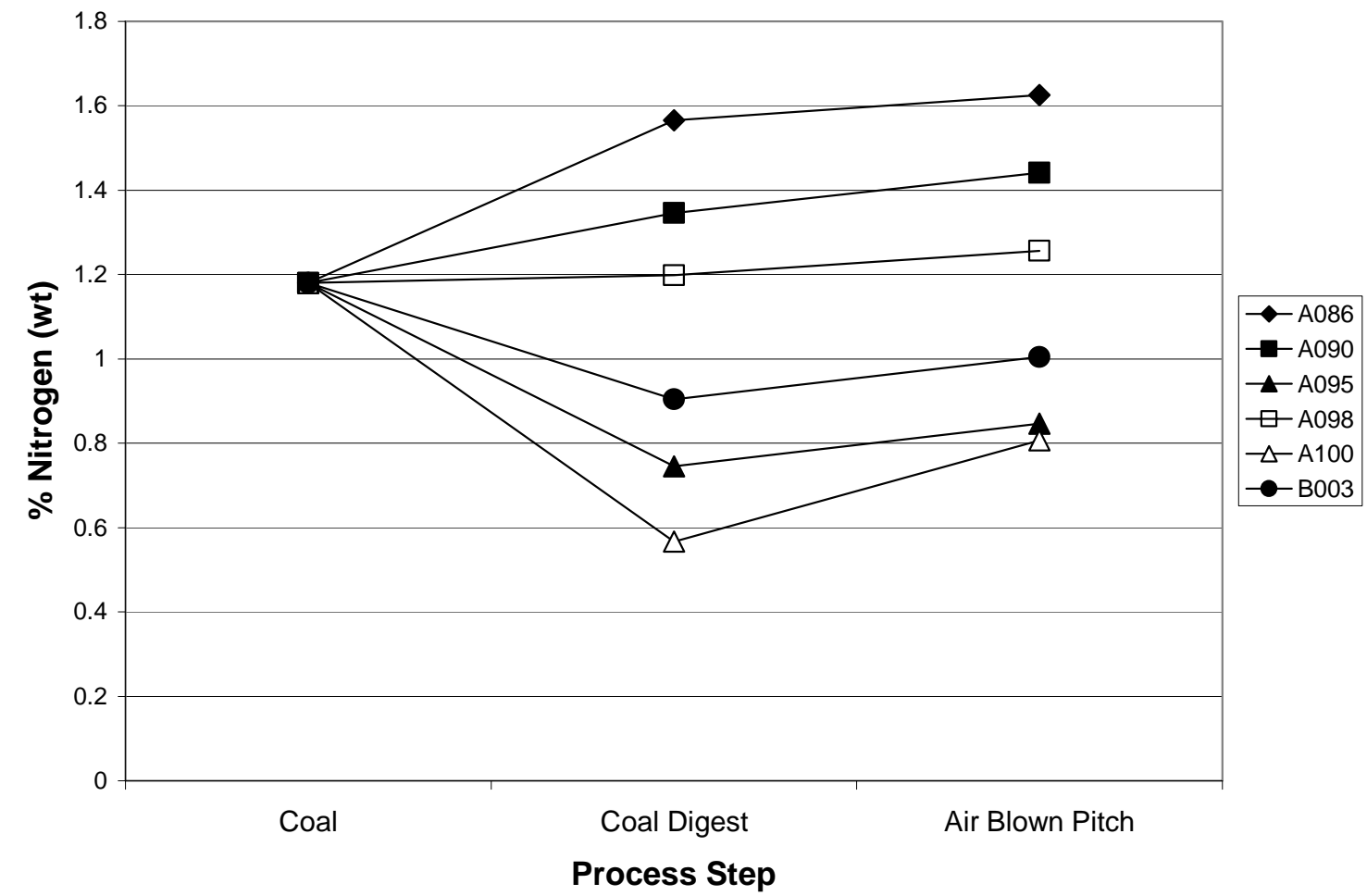

Figure 4.43 - Nitrogen Concentrations in Digest Processing

The hydrogen concentrations in the process increase dramatically during the digestion, which is the desired result (Figure 4.44). This makes for a better precursor, and even though the carbon concentration in the digests is higher than the feed coal (Figure 4.45), the atomic $\mathrm{C} / \mathrm{H}$ ratio is still lower in the digests. After air blowing, the pitches show a reduced concentration of hydrogen, and this is likely due to the distilling of lower molecular weight compounds and the cross-linking that takes place during airblowing. The desired pitch should have a higher $\mathrm{C} / \mathrm{H}$ atomic ratio than the feed material, and this is what increases properties like softening point, coke yield, viscosity, etc. 


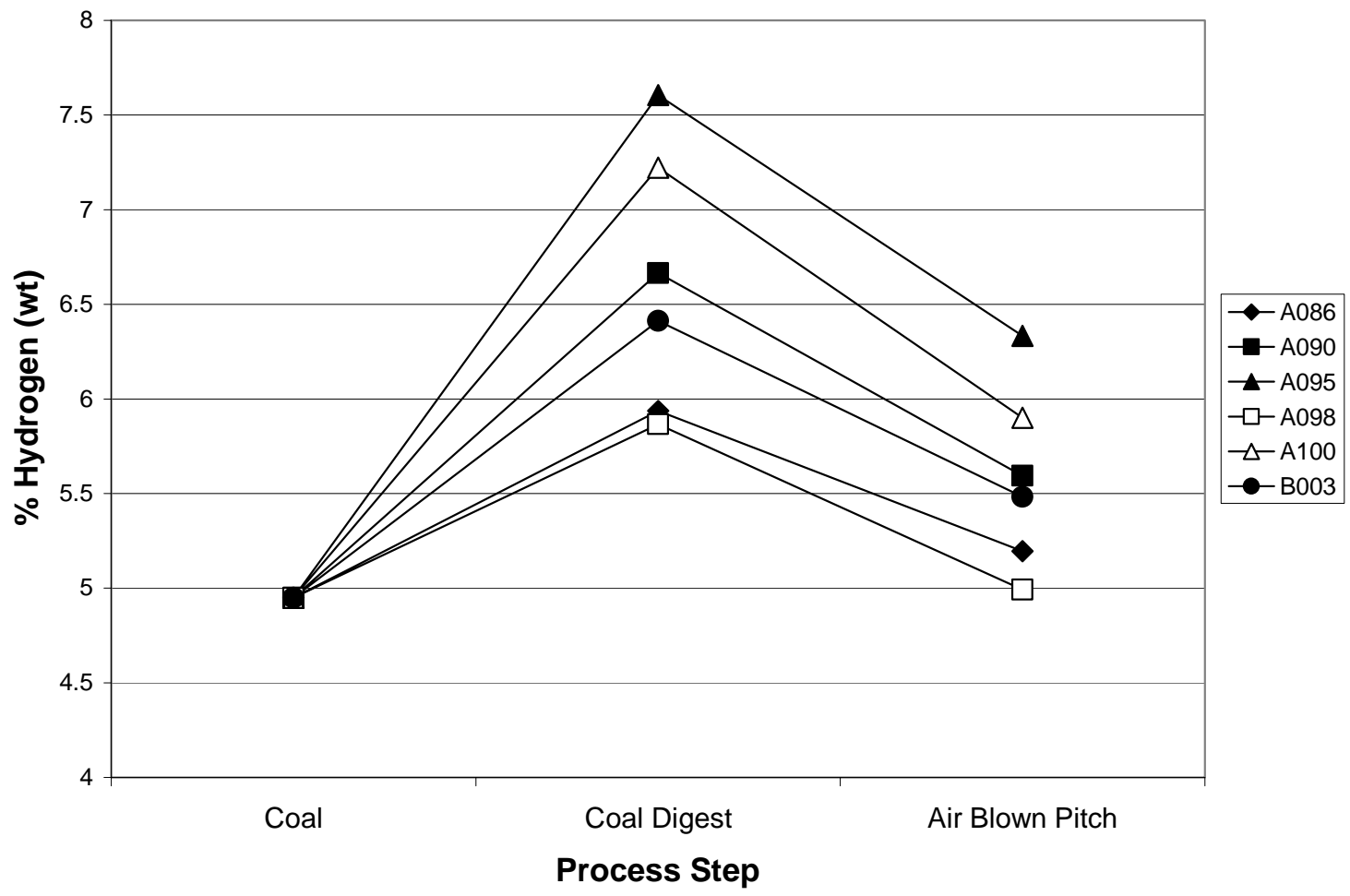

Figure 4.44 - Hydrogen Concentrations in Digest Processing

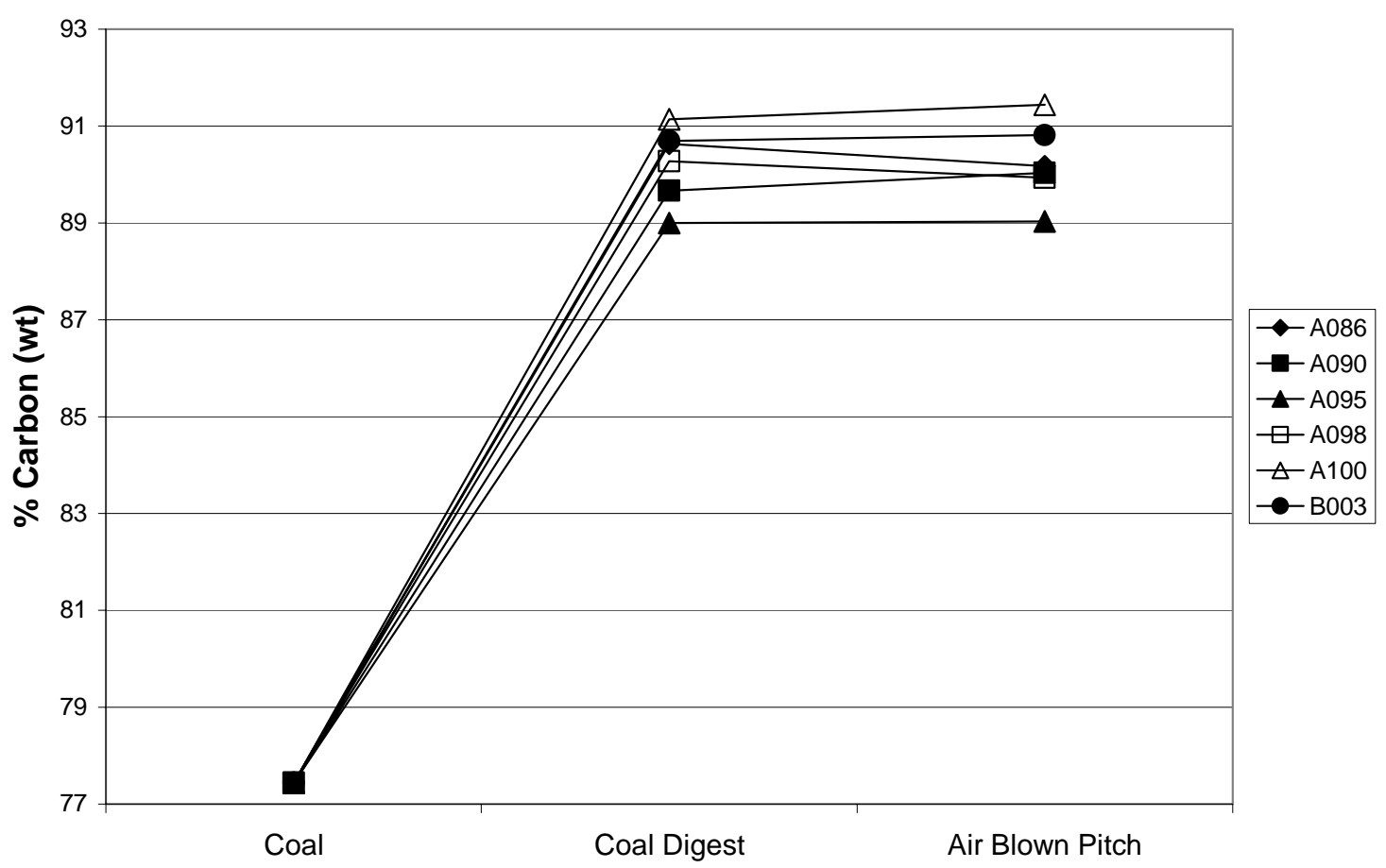

Process Step

Figure 4.45 - Carbon Concentrations in Digest Processing 
The atomic $\mathrm{C} / \mathrm{H}$ ratio and the types of hydrogen and carbon present in the solvents and coal digest liquids may have a significant influence on some of the physical properties of the products. An increase in the atomic $\mathrm{C} / \mathrm{H}$ ratio of the solvent yields an increase in the final softening point of the air-blown digest. The ratio of aromatic hydrogen content to total hydrogen content (the "aromaticity factor") versus the softening point show that as the aromaticity of the solvent increases, so does the softening point of the air-blown digest (Figure 4.46-47). Thus, high concentrations of carbon and aromatic hydrogen in a solvent should lead to a high softening point pitch. For a lower softening point pitch (for instance, a binder pitch), the opposite should hold true. These results are most encouraging, since even though the solvents used are derived from both coal and petroleum, and have many differences, the results are relatively consistent.

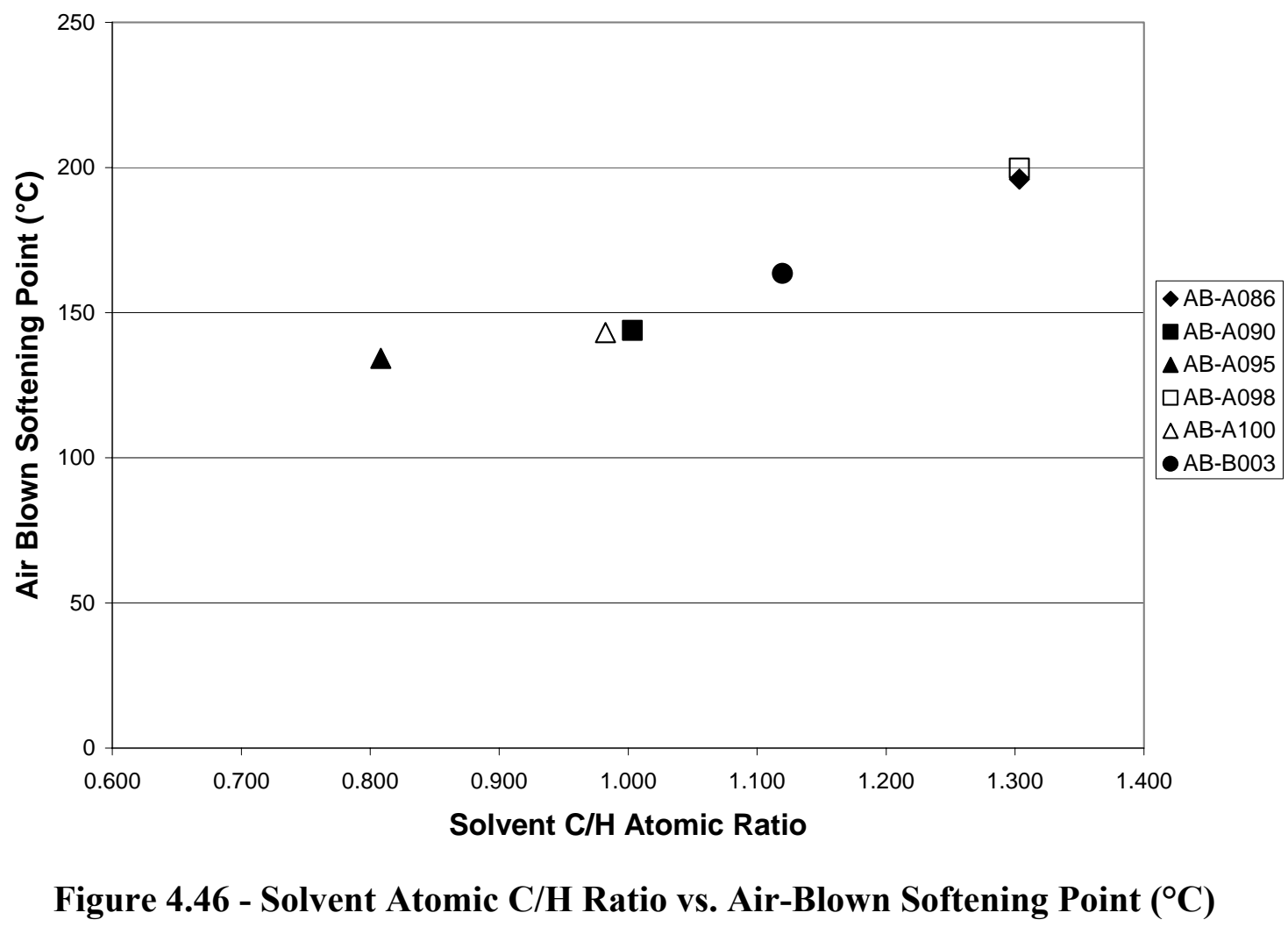




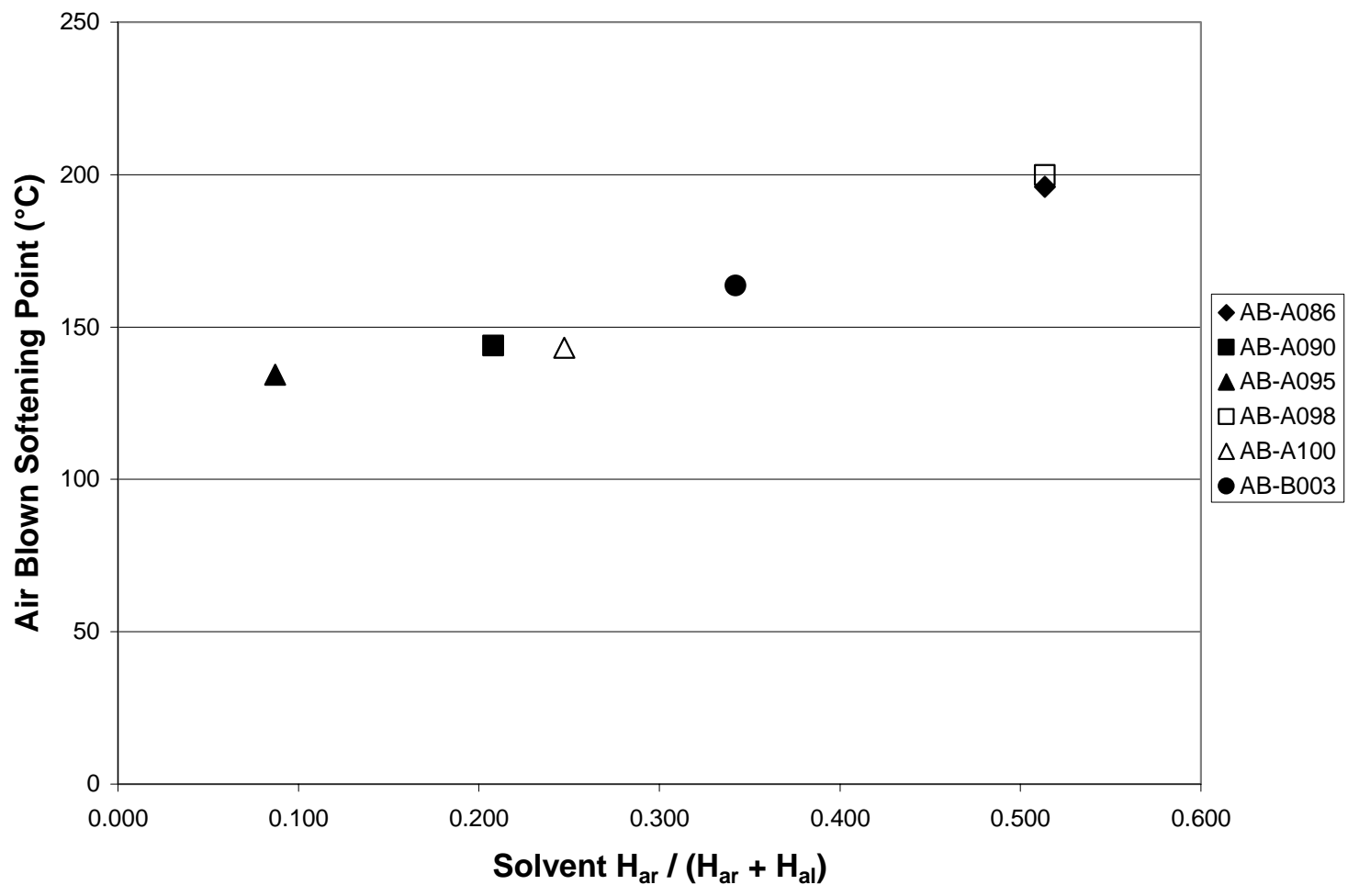

Figure 4.47 - Solvent Aromaticity vs. Softening Point $\left({ }^{\circ} \mathrm{C}\right)$

The tendency for materials with higher aromaticities and $\mathrm{C} / \mathrm{H}$ atomic ratios giving higher softening point air-blown materials carries over into the coal digest liquids. Examination of the coal digest $\mathrm{C} / \mathrm{H}$ atomic ratio vs. air-blown softening point shows nearly the same relationship as with the solvent. The aromaticity of the coal digest liquids also influences the air-blown softening points. As with the atomic $\mathrm{C} / \mathrm{H}$ ratio, as the aromaticity of the digests increases, so too do the air-blown softening points (Figures 4.48-49). 


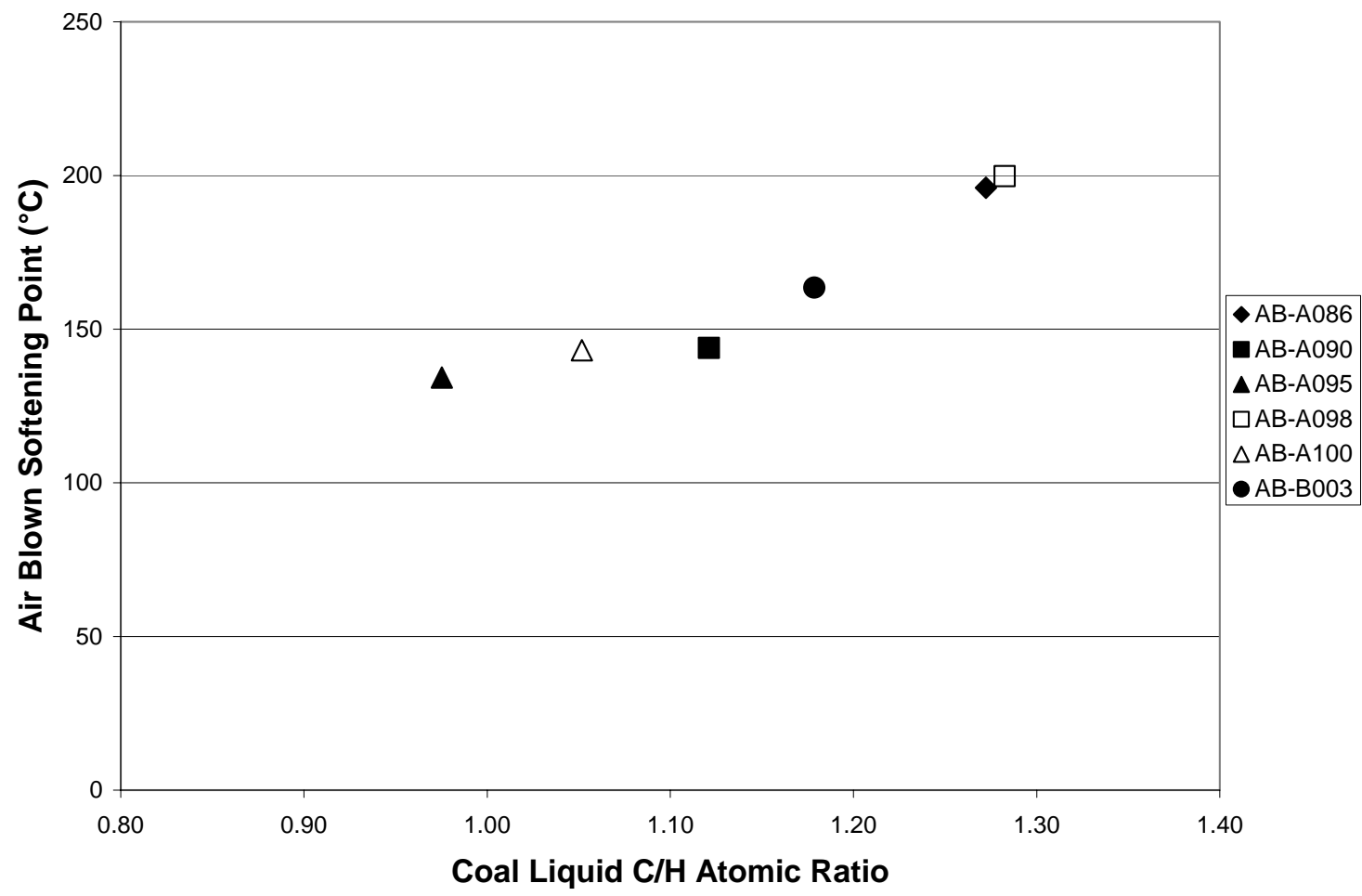

Figure 4.48 - Coal Digest C/H Atomic Ratio vs. Air-Blown Softening Point $\left({ }^{\circ} \mathrm{C}\right)$

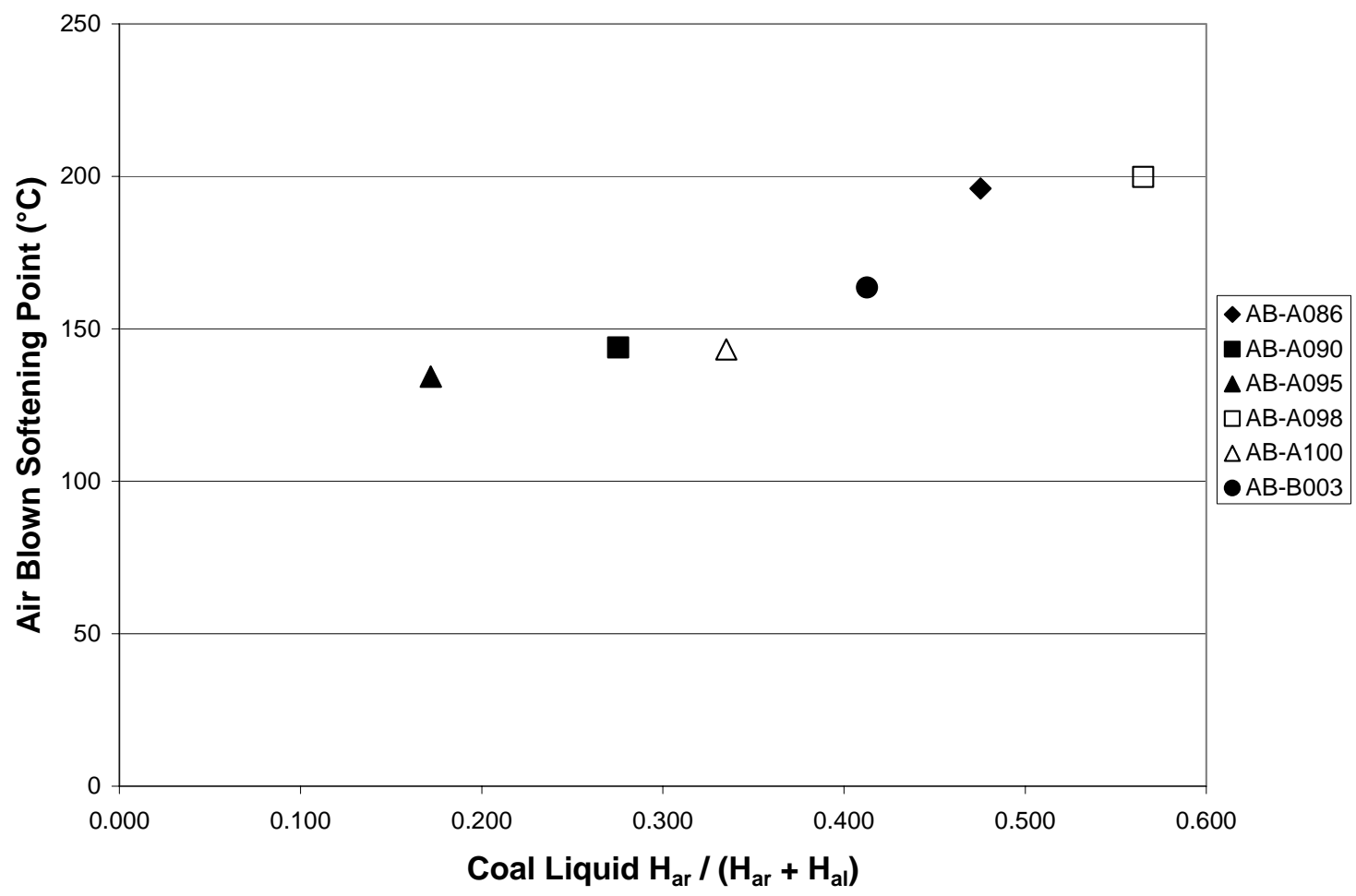

Figure 4.49 - Coal Digest Aromaticity Factor vs. Air-Blown Softening Points $\left({ }^{\circ} \mathrm{C}\right)$ 


\subsection{Vacuum Distillation of Coal Digest Liquids}

While most of the results of the 1-gallon scale-up studies are positive, and even though air-blowing in the past has proven an effective method of altering pitch properties, the coke precursors produced in this study are not affected by the air-blowing in as positive a manner. The process has effectively eliminated the possibility of using the airblown digests as coke feeds, since the cokes made from them are mostly isotropic in nature or very nearly glassy. This observation is in sharp contrast to King ${ }^{(4)}$, who showed that air blowing had little affect on diminishing the optical microstructure. The reason could be the presence of the solvent in the coal digest. King had worked with pitches in which the low molecular weight material was absent.

Vacuum distillation on the coal digest liquids was performed to remove most of the solvent. The use of vacuum distillation is based on the theory that part of the problem with the coal digest liquids is that there is too much solvent still left in the digest. This solvent is presumably much more reactive to air-blowing than the converted coal matter, and cross-links more rapidly so that only isotropic, glassy, carbons are formed upon carbonization.

A sample of each coal digest liquid was vacuum distilled, until about half of the original sample volume was distilled off. As this was still at temperatures below $300^{\circ} \mathrm{C}$, any distillate should be mostly or completely made up of the original digestion solvent, and since the original solvent to coal weight ratio was $2.5: 1$, this process lowers this ratio to approximately $0.75: 1$. The bottoms should be mostly converted coal with some entrained solvent. The details of the distillations are given in Table 4.5. After the distillation, the bottoms, all now solids at room temperature were weighed, and a sample 
of each was carbonized, using the WVU Coke process. The softening point of each one was found, and these along with the coke yield results are shown in Figure 4.50-51. The softening points of the three of these vacuum distilled coal digests are more in line with the production of a binder pitch, approximately $110^{\circ} \mathrm{C}$ (A086, A100 and B003). The vacuum distillation does a much better job of increasing the coke yields of the coal digests. Also, the WVU cokes of these vacuum distilled coal digests were examined under the microscope, and photomicrographs of each are shown next to the corresponding air-blown coal digest cokes (Figures 4.52-56). As can be seen, the vacuum distilled cokes are more anisotropic than the air-blown coal digest cokes. This shows that vacuum distilling some of the solvent off of the coal digests will produce a good pitch precursor, and potentially a coke feed. These vacuum distillations were done at the end of this study, and more research should be conducted to better characterize the types of cokes that could be made when combining vacuum distillation and air-blowing.

Table 4.5 - Details of Vacuum Distillation of Coal Digests

\begin{tabular}{|r|c|c|c|c|c|c|}
\hline & A086 & A090 & A095 & A098 & A100 & B003 \\
\hline Sample Wt. (g) & 670.78 & 514.10 & 521.52 & 510.91 & 512.38 & 516.69 \\
Max Temp. ('C) & 195 & 260 & 275 & 235 & 231 & 225 \\
Bottoms Wt. (g) & 320.05 & 345.40 & 296.15 & 212.04 & 141.29 & 235.08 \\
Distillate Wt. (g) & 350.73 & 279.88 & 252.85 & 292.37 & 277.00 & 268.38 \\
\hline
\end{tabular}




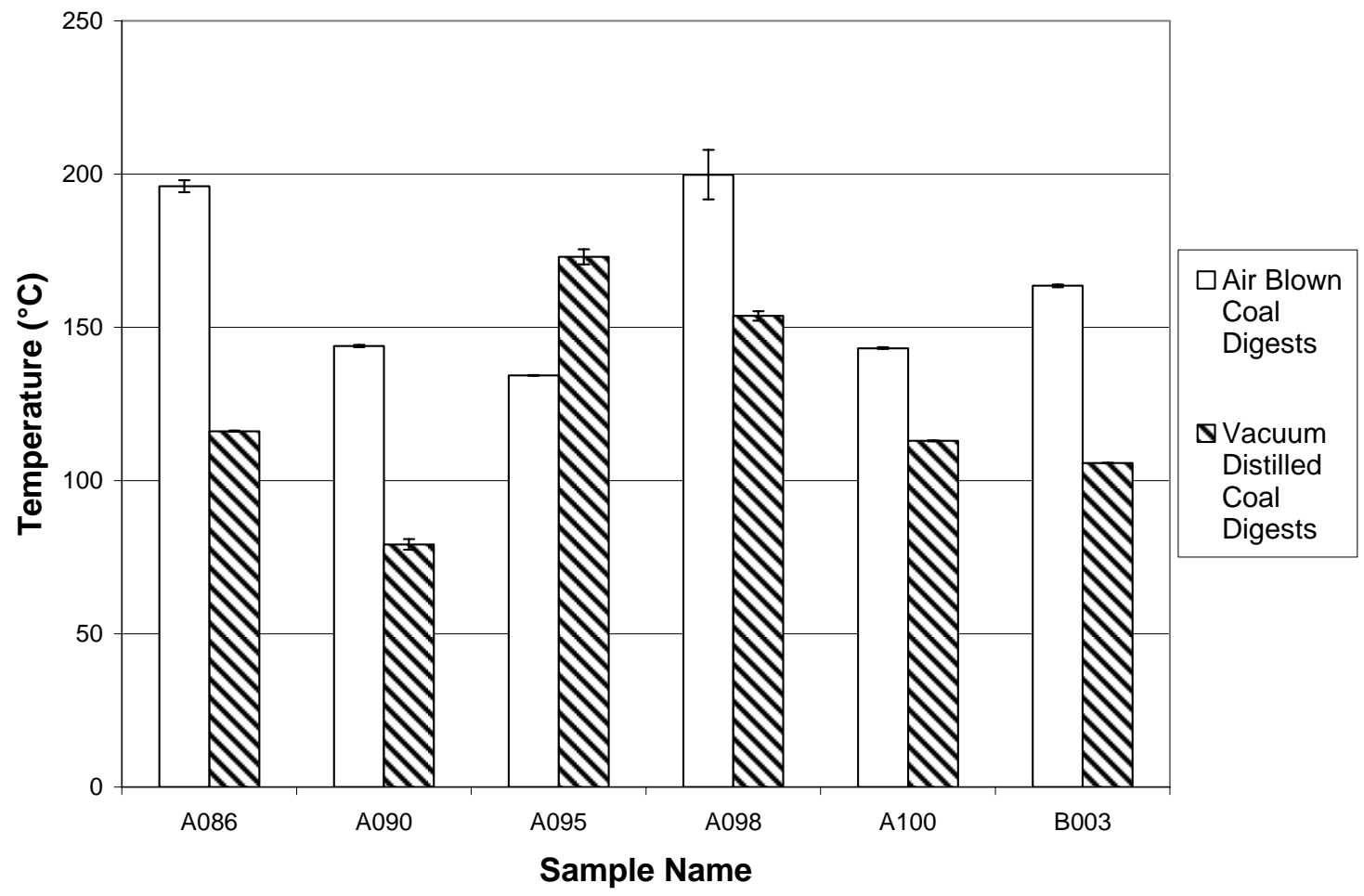

Figure 4.50 - Softening Point $\left({ }^{\circ} \mathrm{C}\right)$ for Air Blown and Distilled Digests

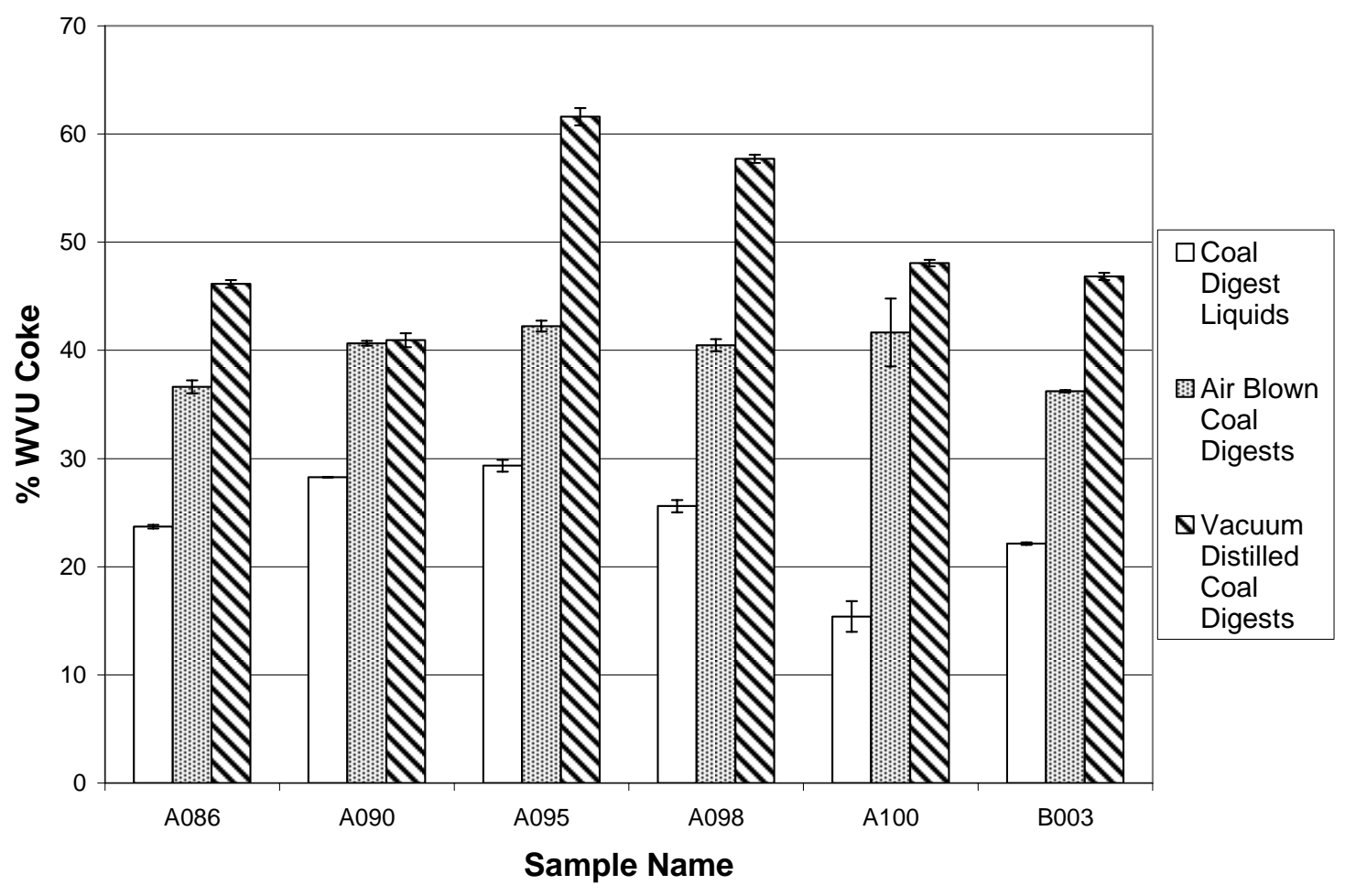

Figure 4.51 - WVU Coke Yield for Liquid, Air-Blown, and Vacuum Distilled Digests 


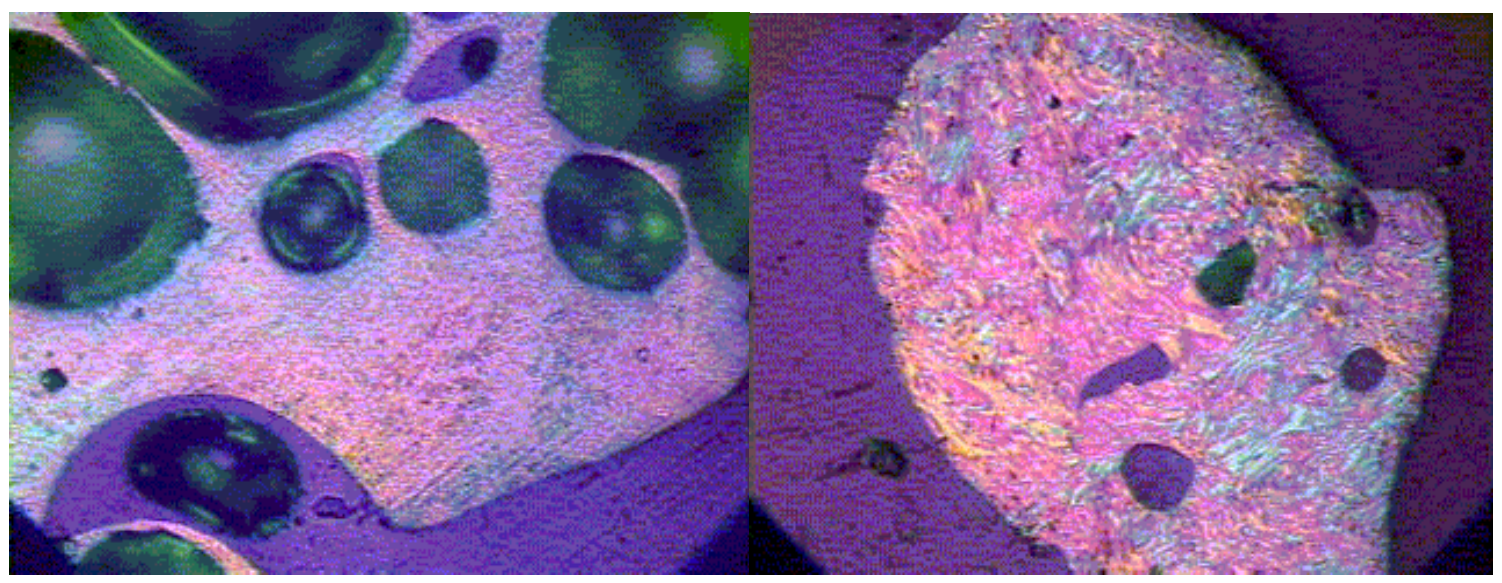

Figure 4.52 - Photomicrographs (160X) of green cokes made from A090 air-blown coal digest (left) and vacuum distilled A090 coal digest (right)

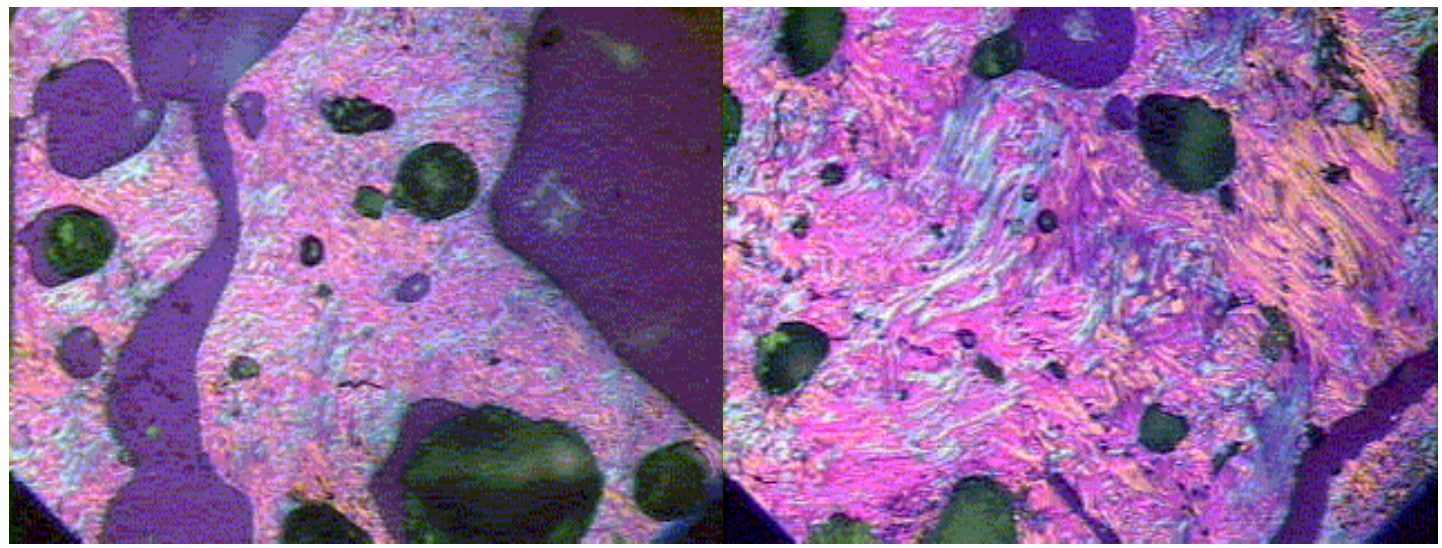

Figure 4.53 - Photomicrographs (160X) of green cokes made from A095 air-blown coal digest (left) and vacuum distilled A095 coal digest (right)

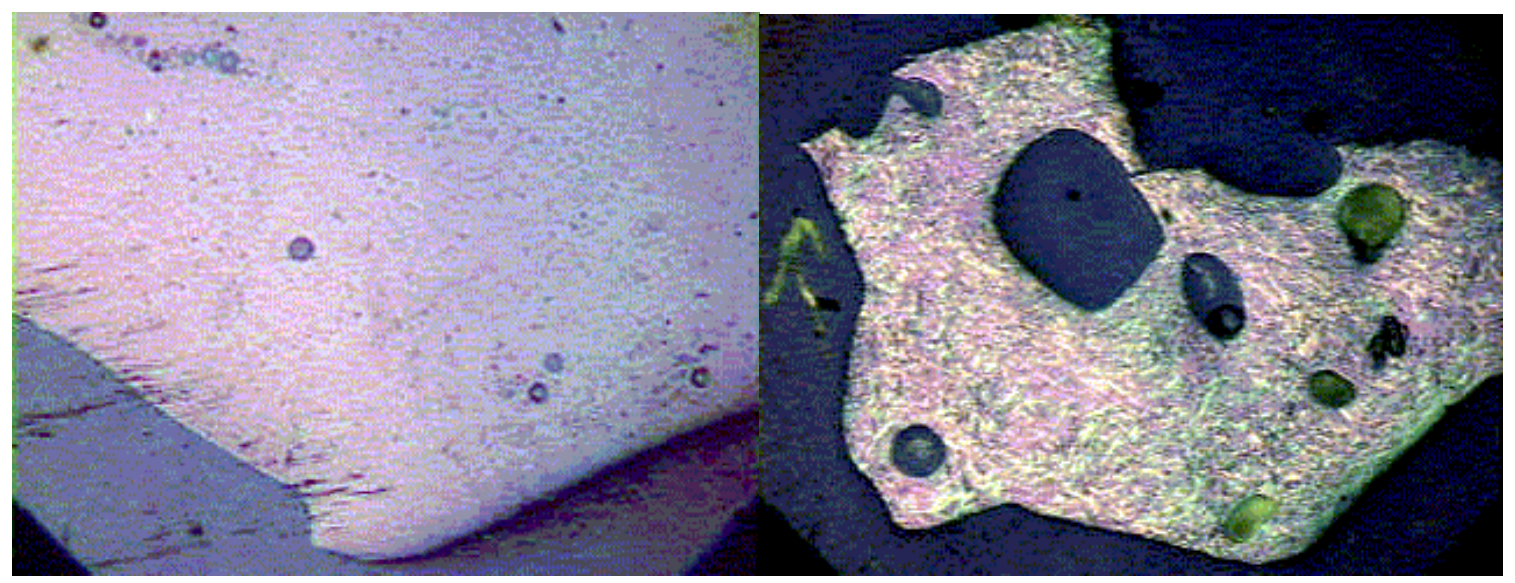

Figure 4.54 - Photomicrographs (160X) of green cokes made from A098 air-blown coal digest (left) and vacuum distilled A098 coal digest (right) 


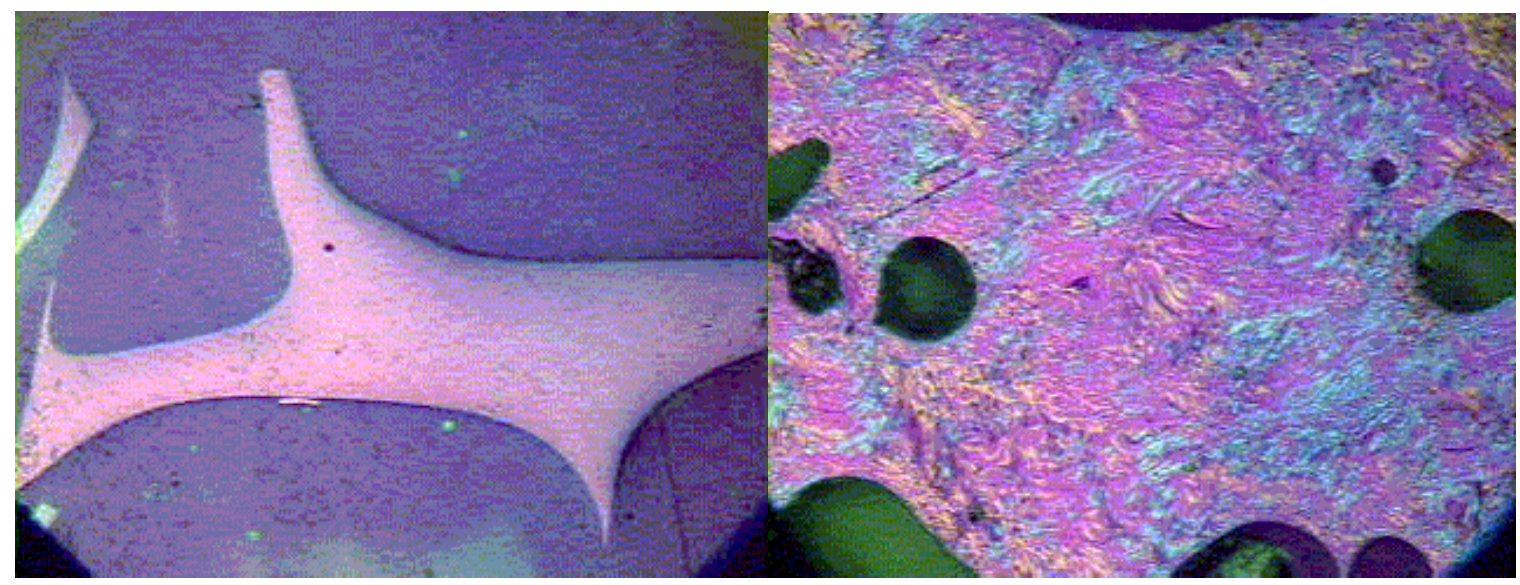

Figure 4.55 - Photomicrographs (160X) of green cokes made from A100 air-blown coal digest (left) and vacuum distilled A100 coal digest (right)

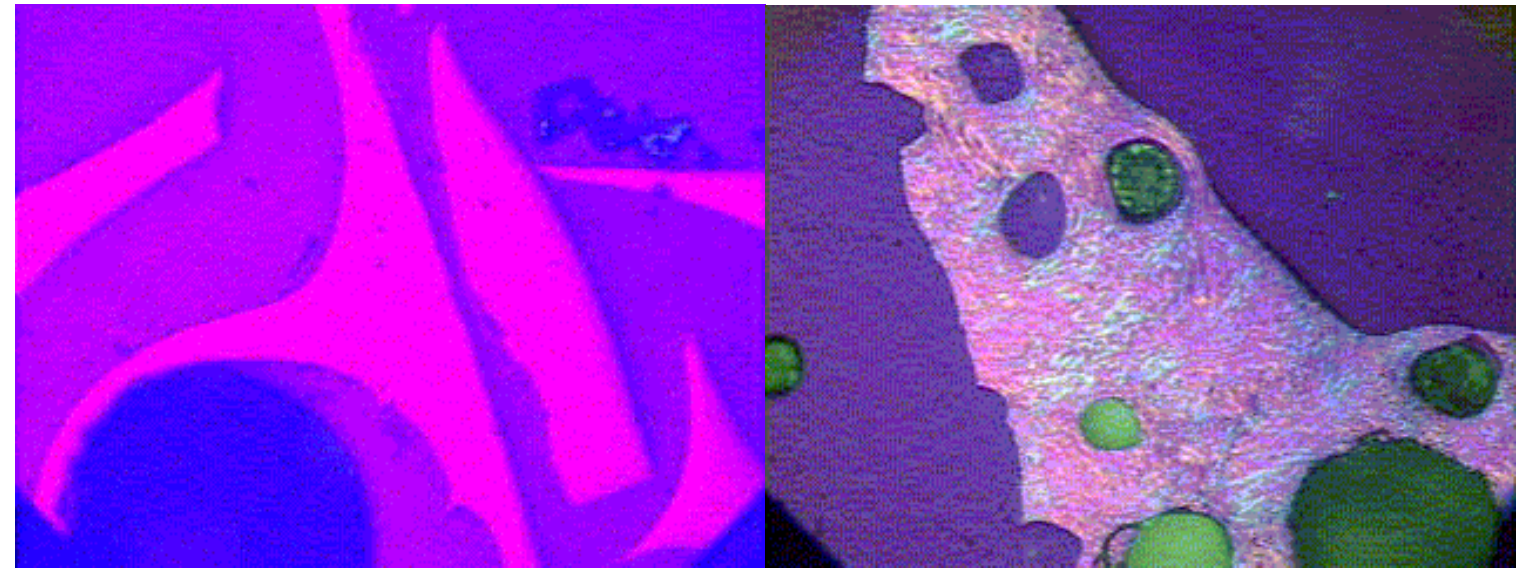

Figure 4.56 - Photomicrographs (160X) of green cokes made from B003 air-blown coal digest (left) and vacuum distilled $\mathrm{B} 003$ coal digest (right) 


\section{CHAPTER 5 - CONCLUSIONS AND RECOMMENDATIONS}

The objective of this thesis study was to develop a method for producing coke feeds suitable for use in carbon anodes by digesting coal with by-product aromatic oils. This digest would then be air-blown to increase the coke yield, and the resultant product could be carbonized into anode-grade coke. Four solvents (2 coal derived and 2 petroleum derived) were chosen, and used both raw and catalytically hydrogenated. The effectiveness of the solvents, best digestion conditions, effectiveness of air-blowing, and the quality of the coal digest liquids and air-blown coal digests were all evaluated. An initial scale-up was also conducted to determine if any changes in the process would be required. From this work, the following conclusions are drawn:

\subsection{CONCLUSIONS}

1. Of the four raw solvents selected, the carbon black base (CBB) worked best when compared to the standard solvent, tetralin. In the parametric studies using the tubing-bomb mini-reactors, the CBB worked the best, followed by the slurry oil (SO), maraflex oil (MO) and finally the anthracene oil (AO).

2. Solvent hydrogenations were conducted using a Ni/Mo catalyst support in a 5gallon stirred tank autoclave. These hydrogenations did use hydrogen gas under pressure, but the conditions for the reaction (temperature from $275-375^{\circ} \mathrm{C}$ and cold $\mathrm{H}_{2}$ pressure from 500-750 psig) were relatively mild. These "mild" hydrogenations only added up to $0.24 \%$ wt of hydrogen to the solvent. Each solvent likely requires its own hydrogenation conditions to be optimal. 
3. Of the three solvents (CBB, SO, MO) that were hydrogenated, the best results were still obtained by the hydrogenated CBB (HCBB-L3). However, the combinations of $\mathrm{CBB}$ variants with SO variants did give comparable conversions of coal as the HCBB-L3 alone. There was only a slight increase in conversion between HCBB-L2 and L3.

4. The slurry oil solvents (SO, HSO-L3) seemed unable to completely solvate the coal during digestion, leading to chunks forming in the reactors. This effect was diminished greatly when slurry oils were combined with the CBB and HCBB's, implying the CBB solvents had a much greater solvation ability, but the mixture still possessed an adequate amount of labile, or donatable, hydrogen.

5. The best conditions for digestion, determined from the parametric studies, was a temperature of $400^{\circ} \mathrm{C}$, digestion time of 1 hour and a reactor pressure of 0 psig after nitrogen purge.

6. Scale up to a 1-gallon autoclave reactor provided better mixing and more thorough conversion of the coal, with conversions as high as 96\% (daf). Again, use of the HCBB-L3 provided the best conversion of coal. Use of the slurry oil (HSO-L3) actually resulted in a lower than predicted conversion. It is believed this is due, again, to the inability of the slurry oil to keep the coal dissolved, even in this higher volume reactor. It was also discovered that reaction at $400^{\circ} \mathrm{C}$ in the 1-gallon reactor was not optimal, and does not properly digest the coal. Airblown digests made from this material were slightly rubbery, thus the temperature of the digestion reaction was raised to $425^{\circ} \mathrm{C}$, which seemed to diminish the rubberiness. 
7. Centrifugation, performed on the products of the 1-gallon digestions, adequately removed any unconverted coal and mineral matter, leaving coal digests with ash values as low as $0.35 \%$. One case, using hydrogenated Maraflex oil, HMO-L3, resulted in a coal digest with a negligible amount of ash material. This is likely due to the low viscosity of the starting solvent, thus improving the efficiency of centrifugation. However, most centrifugations were performed at lower temperatures, due to equipment limitations. Higher temperature separations in this step could result in better reduction in ash content.

8. Coal digests typically had reduced sulfur content, likely through the removal of pyritic sulfur, since upon air blowing the sulfur concentrations rose due to loss of light distillates during the process. Sulfur concentrations down in the tenths of a percent were achievable. However, use of slurry oil as a solvent introduces a significant amount of sulfur to the digest, and as this was the most difficult to process and achieved the lowest conversion, thorough removal of sulfur was not obtained in this run.

9. Air-blowing of the coal digests did result in pitch-like products. Softening points were raised, and the coke yields of the coal digests were increased as well. However, upon examination of the coke microstructures, it became apparent that these were not suitable anode coke feeds. The cokes made from the air-blown digests were almost completely isotropic, even glassy. This is likely due to the presence of too much solvent in the digest. These solvent compounds are probably more responsive to air-blowing than the coal, thus cross linking occurs 
with the solvent compounds. This prevents the development of any anisotropy in the cokes, and thus eliminating air-blown digests from use as anode coke feeds.

10. In an attempt to solve this situation, vacuum distillation of the coal digest liquids was used. After distillation of half of the volume of a sample of the coal digest liquids, the remaining material was then coked and tested for softening point. The coke yields for these vacuum distilled coal digests were even better than the airblown counterparts, but more importantly, examination of the coke microstructures showed the development of more anisotropic cokes. This is the desired result, and these materials may become suitable feeds for anode cokes.

11. The softening points of three of these vacuum distilled coal digests (VD-A086, VD-A100, and VD-B003) were in the range of a binder pitch (app. $110^{\circ} \mathrm{C}$ ). This is another possible application for these materials, provided the proper control of the softening point and coke yields.

\subsection{RECOMMENDATIONS FOR FUTURE WORK}

Based on the results of this thesis study, several recommendations must be made regarding future research work. As the vacuum distilled coal digests seem to provide the most suitable anode coke feed, but the coke yields are still not as high as desired, airblowing of these vacuum distilled digests is recommended. Even though there was a lack of success in air-blowing the coal digests, it is believed this is simply due to the presence of too much solvent in the digest. Lower solvent to coal ratios would likely reduce the conversion obtained in the digestion, so vacuum distillation after digestion should be used. However, once this is done, these bottoms, which are already much more pitch 
like, could be air-blown to improve the coke yields. These products are more like the pitches studied by N. King ${ }^{(4)}$ in a study on the effectiveness of air-blowing pitches, and it is believed air-blowing the vacuum distilled digests could result in a very high quality anode coke feed.

The level of hydrogenation chosen in this study was somewhat arbitrary, although the results seem to indicate the level 3 hydrogenation is more than adequate for this process. However, optimization of this has not been conducted, and according to Figure 4.22 and 4.23, level three hydrogenated CBB is not necessarily the lone best case. The optimal hydrogenation conditions need to be determined through a parametric style study, varying temperature, cold hydrogen pressure, reaction time, and even solvent or solvent combination. Each solvent or solvent combination may have a different set of optimal hydrogenation conditions. Care must be taken to control the amount of hydrogenation performed on solvents, as excessive pretreatment can result in the production of alicyclics that are poor $\mathrm{H}$-donors in comparison to aromatics.

While a temperature of $400^{\circ} \mathrm{C}$ was the best during the parametric studies, this was not the case after scale-up. More work could be done on the tubing bomb scale on temperature, and it may be worthwhile to investigate using even higher temperatures in the digestions. Optimum conversion may be observed at temperatures around 400$425^{\circ} \mathrm{C}$, but higher temperatures would induce retrograde reactions and may result in materials that would be more useful as coke feeds.

Compositional analysis of the off-gas from the solvent hydrogenations (via gas chromatography) would provide more insight on what is happening differently with each 
solvent during hydrogenation (i.e. CBB vs. SO). Gas samples were obtained, but analysis was not possible at the time.

Continued detailed chemical characterization of the solvents and coal digest liquids is highly recommended, especially through the use of proton n.m.r. or $\mathrm{C}^{13}$ n.m.r or molecular weight distributions. These analyses were not used in this study due to the lack of availability of an effective solvent to use in the NMR and lack of equipment for molecular weight distribution determination.

Additional work should include additional blending of solvents. It was apparent during the parametric studies that a mixture of the raw CBB and the HCBB-L2 performed nearly as well as the HCBB-L3 in the digestion. If only half of the solvent in the process had to be pre-hydrogenated, this would improve the process economics.

As the ash content of the coal liquids was not low enough for anode coke use, higher temperature solid-liquid separations should be employed after the digestion step. This will significantly lower the viscosity of the coal liquid, allowing for a more thorough removal of mineral matter and unconverted coal matter.

More work could be conducted on varying the time and temperature used during the air-blowing step, especially if this is employed after vacuum distillation. Additionally, the coal liquids could be carefully air-blown at low temperatures and times to produce a material that could be used as a binder pitch.

Pilot scale studies of this process (coal digestion, solvent removal, air-blowing) should be conducted at some point. This process can very easily be scaled up to the pilot scale, and the data obtained in this study (such as conversions, $\mathrm{T}$ and $\mathrm{P}$ data, and viscosities) will be valuable in the development of this pilot scale facility. 


\section{REFERENCES}

1. Song, C. and Schobert, H. H., Fuel, 75(6), 724-736, 1996.

2. Marsh, $\mathrm{H}$ and Fiorino, K. “The Manufacture of Carbon Anodes for Aluminum Production” Introduction to Carbon Technologies. Universidad de Alicante, Secretariado de Publicaciones, p 537, 1997.

3. Redmount, M.B. and Heintz, E.A. “The Manufacture of Graphite Electrodes” Introduction to Carbon Technologies. Universidad de Alicante, Secretariado de Publicaciones, p 519, 1997.

4. King, Nathan. Chemical and Physical Modification of Petroleum, Coal-tar, and Coal-extract Pitches by Air Blowing. Thesis, Department of Chemical Engineering, West Virginia University, Morgantown, WV, 2004.

5. Marsh, H. and Cornford, C. “Mesophase: The Precursor to Graphitizable Carbon” Petroleum Derived Carbons. Ed. Deviney, M.L. and O’Grady, T.M. ACS Symposium Series. American Chemical Society, Washington, D.C., p 266, 1976.

6. Fenton, D., Direct Liquefaction of Coal with Coal-Derived Solvents to Produce Precursors for Carbon Products. Thesis, Department of Chemical Engineering, West Virginia University, Morgantown, WV, 2001.

7. Hanson, W. E., “Nomenclature and Terms,” Bituminous Materials: Asphalts, Tars, and Pitches, Vol. I, Arnold J. Hoiberg, ed., Robert E. Krieger Publishing Company, Huntington, NY, 1979.

8. Gray, R.J. and Krupinski, K.C. "Pitch Production: Supply, coking, optical microscopy and applications.” Introduction to Carbon Technologies. Universidad de Alicante, Secretariado de Publicaciones, p 358, 1997. 
9. Newman, J.W. and Newman, K.L. "A History of Pitch Technologies.” Introduction to Carbon Technologies. Universidad de Alicante, Secretariado de Publicaciones, p 285, 1997.

10. "Final Technical Report”. Coal Based Nuclear Graphites for the New Production Gas Cooled Reactor. Task 1: Development of Coal-Derived Isotropic Coke and Nuclear Graphite. West Virginia University, 1994.

11. Yamaguchi, Chiharu; Mondori, Juji; Matsumoto, Akira; Honma, Hidekazu; Kumagai, Haruo; Sanada, Yuzo. “Air-blowing reactions of pitch: I. Oxidation of aromatic hydrocarbons.” Carbon, 33(2), 193-201, 1995

12. Maeda, Toyohiro; Zeng, Shu Ming; Tokumitsu, Katsuhisa; Mondori, Juji; Mochida, Isao. "Preparation of isotropic pitch precursors for general purpose carbon fibers (GPCF) by air blowing - I. Preparation of spinnable isotropic pitch precursor from coal-tar by air blowing.” Carbon, 31(3), 407-12, 1993.

13. Zeng, Shu Ming; Maeda, Toyohiro; Tokumitsu, Katsuhisa; Mondori, Juji; Mochida, Isao. "Preparation of isotropic pitch precursors for general purpose carbon fibers (GPCF) by air blowing - II. Air blowing of coal-tar, hydrogenated coal-tar, and petroleum pitches.” Carbon, 31(3), 413-19, 1993.

14. Fernandez, J. J.; Figueiras, A.; Granda, M.; Bermejo, J.; Menendez, R. "Modification of coal-tar pitch by air-blowing - I. Variation of pitch composition and properties.” Carbon, 33(3), 295-307, 1995.

15. Menendez, R.; Fleurot, O.; Blanco, C.; Santamaria, R.; Bermejo, J.; Edie, D. "Chemical and rheological characterization of air-blown coal-tar pitches." Carbon, 36(7-8), 973-979, 1998. 
16. Choi, J. H.; Kumagai, H.; Chiba, T.; Sanada, Y. “Carbonization of pitches in air blowing batch reactor.” Carbon, 33(2), 109-14, 1995.

17. Schobert, H. Coal: The Energy Source of the Past and Future. American Chemical Society, USA, 1987.

18. Adams, H.A. “Delayed Coking: Practice and Theory.” Introduction to Carbon Technologies. Universidad de Alicante, Secretariado de Publicaciones, p 491, 1997.

19. Stopes, M.C., Proc. R. Soc. London, 90B, 470, 1919.

20. Wiser, W. Preprints Fuel Division ACS Meeting, 20 (2), 122, 1975.

21. Ergun, S., “Coal Classification and Characterization,”. Coal Conversion Technology. Ed. C.Y.Wen and E.S. Lee, Addison-Wesley Publishing Company, Reading, Mass., 1979.

22. Webster's New Collegiate Dictionary, Random House Publising, New York, 1991.

23. Merrick, D. Coal Combustion and Conversion Technology. Elsevier. New York, 1984.

24. Speight, J.G. The Chemistry and Technology of Coal. Marcel Dekker. New York, 1983

25. Curran, G.P., Struck, R.T., and Gorin, E., Ind. Eng. Chem. Process Des. Dev., 6, 166, 1967.

26. Vernon, L.W., Fuel, 59, 102, 1980.

27. Wiser, W. H., Fuel, 47, 475, 1968.

28. Malhotra, R. and McMillen, D.F., Energy \& Fuels, 4, 184, 1990. 
29. Malhotra, R. and McMillen, D.F., Energy \& Fuels, 7, 227, 1993.

30. Berkowitz, N. An Introduction to Coal Technology. Academic Press, Inc., San Diego, 1994.

31. Virk, P.S., Fuel. 58, 149-151, 1979.

32. Farcasiu, M., Mitchell, T.O., and Whitehurst, D.D., ACS Div. Fuel Chem., Prepr. 27 (7), 11, 1976.

33. Fisher, C.H., Sprunk, G.C., Eisner, A., O’Donnell, H.J., Clarke, L., and Storch, H.H., Hydrogenation and Liquefaction of Coal: Part 2-Effect of Petrographic Composition and Rank of Coal.” U.S. Bureau of Mines Technical Paper, 642, 1942.

34. Keogh, R.A. and Davis, B.H., Energy \& Fuels, 8, 289, 1994.

35. Keogh, R.A., Taulbee, D.N., Hower, J.C., Chawla, G., and Davis, B.H., Energy \& Fuels, 6, 614, 1992.

36. Cloke, M. and Wang, C., Energy \& Fuels, 9, 560, 1995.

37. Given, P.H., Cronauer, D.C., Spackmann, W., Lovell, H.L., Davis, A., and Biswas, B. "Dependence of Coal Liquefaction Behavior on Coal Characteristics. 2. Role of Petrographic Composition.” Fuel, 54 (1), 40-49, 1975b.

38. Huang, H., Wang, K., Wang, S., Klein, M.T., and Calkins, W.H., Energy \& Fuels, 12, 95, 1998.

39. Whitehurst, D.D., Mitchell, T.O., and Farcasiu, M., Coal Liquefaction: The Chemistry and Technology of Thermal Processes, Mobil Research and Development Corporation, Central Research Division, Princeton, New Jersey, 1980. 
40. Shibaoka, M. Fuel, 60, 240-246., 1981b.

41. Miller, R.L., Silver, H.F., and Hurtubise, R.J., Ind. Eng. Chem. Process Des. Dev., 21, 170-173, 1982.

42. Davies, G.O., Derbyshire, F.J., and Price, R. J. Inst. Fuel, 121-126, 1977.

43. Oele, A.P., Waterman, H.I., Goedkoop, M.L., and Van Krevelen, D.W., Fuel, 30, 169, 1951.

44. Mukherjee, D.K., and Chowdry, P.B., Fuel, 55, 4, 1976.

45. Yen, Y.K., Furlani, D.E., and Weller, S.W., Ind. Eng. Chem. Prod. Res. Dev., 15, 24, 1976.

46. Tomic, J. and Schobert, H.H., Energy \& Fuels, 10, 709, 1996.

47. Finseth, D.H., Cillo, D.L., Sprecher, R.F., Retcofsky, H.L., and Lett, R.G., Fuel, 64, 1718, 1985.

48. Chawla, B., Keogh, R., and Davis, B.H., Energy \& Fuels, 3, 236, 1989.

49. Wilson, M.A., Pugmire, R.J., Vasallo, A.M., Grant, D.M., Collin, P.J., and Zilm, K.W., Ind. Eng. Chem. Prod. Res. Dev., 21, 477, 1982.

50. Curtis, C.W. and Hwang, Jong-Sic. “The Reactivity of Different Coal and Residuum Combinations in Coprocessing.” Fuel Processing Technology, 30, 4767, 1992.

51. Speight, J.G. and Moschopedis, S.E. “The Co-Processing of Coal with Heavy Feedstocks.” Fuel Processing Technology, 13, 215-232, 1986.

52. Artia, s., Osafune, K., Yamada, Y., Kakiyami, H., Honda, H., Tagawa, N., and Abe, S. Nenryo Kyokai Shi, 19, 1060, 1977. 
53. Orr, Edward C, Yanlong Shi, Lian Shao, Jing Liang, Weibing Ding, Larry L. Anderson, Edward M. Eyring. "Waste Oils used as Solvents for Different Ranks of Coal.” Fuel Processing Technology, 49, 233-246, 1996.

54. Wallace, S., Bartle, K.D., Kemp, W., Steedman, W., Flynn, T., Burke, M.P., Jones, C.J. and Taylor, N. "Coal Conversion in Co-Processing with Heavy Petroleum Residues.” Fuel Processing Technology, 24, 225-230, 1990.

55. Inukai, Yoshinari. "Hydroliquefaction of Illinois No.6 Coal with Petroleum Atmospheric Residue using Oil-Soluble Molybdenum Catalyst.” Fuel Processing Technology, 43, 157-167, 1995.

56. Wallace, S., Bartle, K.D., Burke, M.P., Egia, B., Lu, S., Taylor, N., Flynn, T., Kemp, W., and Steedman, W. “Characterization of Petroleum Feedstocks for Coal-Oil Co-Processing.” Fuel, 68, 961-967, 8/1989.

57. Kouzu, M., Saegusa, H., Hayashi, T., Nishibayashi, T., Kobayashi, M., Itoh, H., and Hattori, H. "Effect of Solvent Hydrotreatment on Product Yield in the Coal Liquefaction Process.” Fuel Processing Technology, 68, 237-254, 2000.

58. Sato, Y., Yamamoto, Y., Kamo, T., Inaba, A., Miki, K., and Saito, I. “Effect of Hydrotreatment of Various Heavy Oils as Solvent for Coal Liquefaction.” Energy \& Fuels, 5, 98-102, 1991.

59. Miranda, J.L., Palomar, V., Martinez, M.T., and Osacar, J. “Catalytic Hydrogenation of a Spanish Coal: a Study of the Operation Variables.” Fuel, 66, 119-122, 1/1987.

60. Caballero, B.M., de Marco, I., Chomon, M.J., and Legarreta, J.A. “Coal Liquefaction with Anthracene Oil: Influence of Solvent Pretreatment, 
Temperature, Catalyst and Pressure.” Fuel Science and Technology, International, 12, 1067-1079, 1994.

61. Shi, Yanlong, Shao, Lian, Olson, W.F. and Eyring, E.M. “Coprocessing Coal with Hydrogenated Vacuum Pyrolyzed Tire Oil.” Fuel Processing Technology, 58, 135-150, 1999.

62. Townsend, A.T. and Larkins, F.P. "Upgrading of an Australian Coal-Derived Liquid. 2. Characterization of Ni-Mo/ $/ \mathrm{Al}_{2} \mathrm{O}_{3}$ catalysts.” Fuel Processing Technology, 32, 115-131, 1992.

63. Awadalla, A.A., Cookson, D.J., and Smith, B.E. "Coal Hydrogenation: Quality of Start-Up Solvents and Partially Process-Derived Recycle Solvents.” Fuel, 64, 1097-1107, 8/1985. 


\section{APPENDIX A: MASS BALANCES}


Table A1 - Mass balances for Solvent Hydrogenations

\begin{tabular}{|c|c|c|c|c|c|c|c|c|}
\hline $\begin{array}{c}\text { SAMPLE } \\
\text { NAME }\end{array}$ & $\begin{array}{c}\text { MASS } \\
\text { SOLVENT } \\
\text { IN (g) }\end{array}$ & $\begin{array}{c}\text { MASS } \\
\text { SOLVENT } \\
\text { OUT (g) }\end{array}$ & $\begin{array}{c}\text { MASS } \\
\text { LOST } \\
\text { (g) }\end{array}$ & $\begin{array}{c}\text { \% MASS } \\
\text { BALANCE }\end{array}$ & $\begin{array}{c}\text { COLD } \\
\mathbf{H}_{2} \\
\text { (PSIG) }\end{array}$ & $\begin{array}{c}\text { FINALD } \\
\text { COLD } \\
\mathbf{H}_{2} \\
\text { (PSIG) }\end{array}$ & $\begin{array}{c}\mathrm{H}_{2} \\
\text { CONSUMED } \\
(\mathbf{g})\end{array}$ & $\begin{array}{c}\mathrm{H}^{2} \\
\text { CONSUMED } \\
\text { (wt \%) }\end{array}$ \\
\hline HCBB-L1 & 12518.00 & 11934.35 & 583.65 & 95.34 & 500 & 200 & 12.99 & 0.10 \\
\hline HCBB-L2 & 11926.60 & 11889.50 & 37.10 & 99.69 & 480 & 80 & 16.17 & 0.14 \\
\hline HCBB-L3 & 12016.30 & 12007.60 & 8.70 & 99.93 & 750 & 80 & 28.48 & 0.24 \\
\hline HSO-L3 & 12003.27 & 11833.75 & 169.52 & 98.59 & 750 & 100 & 28.64 & 0.24 \\
\hline HMO-L3 & 11577.75 & 11479.13 & 98.62 & 99.15 & 750 & 100 & 28.20 & 0.24 \\
\hline
\end{tabular}

Table A2 - Mass Balances for Parametric Studies

\begin{tabular}{|c|c|c|c|c|c|c|c|c|}
\hline Run Name & $\mathrm{T}\left({ }^{\circ} \mathrm{C}\right)$ & $\begin{array}{c}P \\
\text { (psig) }\end{array}$ & $\begin{array}{c}\text { Wt Coal } \\
\text { In (g) }\end{array}$ & $\begin{array}{c}\text { Wt } \\
\text { Solvent } \\
\text { In (g) }\end{array}$ & $\begin{array}{c}\text { Total In } \\
\text { (g) }\end{array}$ & $\begin{array}{c}\text { THF } \\
\text { Soluble } \\
\text { Digest (g) }\end{array}$ & $\begin{array}{c}\text { THF } \\
\text { Insoluble } \\
\text { Digest (g) }\end{array}$ & $\begin{array}{c}\text { In-Out I } \\
\text { In (\%) }\end{array}$ \\
\hline$\overline{C B B A}$ & 400 & 0 & 3.0122 & 8.1000 & 11.1122 & 9.1433 & 1.9772 & -0.07 \\
\hline CBB B & 400 & 0 & 3.0095 & 8.1754 & 11.1849 & 9.4559 & 1.6658 & 0.57 \\
\hline HCBB-L1 A & 400 & $\overline{0}$ & 3.0019 & 8.1885 & 11.1904 & 9.7366 & 1.4413 & 0.11 \\
\hline HCBB-L1 B & 400 & 0 & 3.0060 & 8.0100 & 11.0160 & 9.5510 & 1.3500 & 1.04 \\
\hline HCBB-L2 A & 400 & 0 & 3.0099 & 8.2010 & 11.2109 & 9.1218 & 1.9664 & 1.09 \\
\hline HCBB-L2 B & 400 & $\overline{0}$ & 3.0115 & 8.2345 & 11.2460 & 9.7291 & 1.1323 & 3.42 \\
\hline HCBB-L3 A & 400 & 0 & 3.0091 & 8.0189 & 11.0280 & 9.8029 & 1.1823 & 0.39 \\
\hline HCBB-L3 B & 400 & 0 & 3.0136 & 8.0097 & 11.0233 & 9.8765 & 1.1393 & 0.07 \\
\hline CBB/HCBB-L2 A & 400 & 0 & 3.0026 & 8.0515 & 11.0541 & 9.8314 & 1.0646 & 1.43 \\
\hline CBB/HCBB-L2 B & 400 & 0 & 3.0050 & 8.1418 & 11.1468 & 10.0869 & 0.9727 & 0.78 \\
\hline AO A & 400 & 0 & 3.0011 & 8.1275 & 11.1286 & 8.3529 & 1.9383 & 7.52 \\
\hline AO B & 400 & 0 & 3.0034 & 7.5074 & 10.5108 & 8.0864 & 1.9030 & 4.96 \\
\hline MO A & 400 & 0 & 3.0001 & 8.0307 & 11.0308 & 9.3717 & 1.7426 & -0.76 \\
\hline MO B & 400 & 0 & 3.0028 & 8.0564 & 11.0592 & 9.5057 & 1.7944 & -2.18 \\
\hline SO A & 400 & $\overline{0}$ & 3.0033 & 8.0368 & 11.0401 & $\mathrm{~N} / \bar{A}$ & 1.4888 & $\mathrm{~N} / \bar{A}$ \\
\hline SO B & 400 & $\overline{0}$ & 3.0105 & 8.0037 & 11.0142 & $\overline{\mathrm{N}} / \overline{\mathrm{A}}$ & 1.4954 & $\bar{N} / \bar{A}$ \\
\hline HSO-L3 A & 400 & 0 & 3.0036 & 8.0365 & 11.0401 & 9.5397 & 1.2420 & 2.34 \\
\hline HSO-L3 B & 400 & 0 & 3.0088 & 8.0304 & 11.0392 & 9.6252 & 1.1606 & 2.30 \\
\hline SO/HCBB-L2 A & 400 & 0 & 3.0076 & 8.0540 & 11.0616 & 9.3377 & 1.1293 & 5.38 \\
\hline SO/HCBB-L2 B & 400 & 0 & 3.0087 & 8.0692 & 11.0779 & 9.4799 & 1.2801 & 2.87 \\
\hline HCBB-L3/HSO-L3 A & 400 & 0 & 3.0014 & 8.0438 & 11.0452 & 9.3669 & 1.2820 & 3.59 \\
\hline HCBB-L3/HSO-L3 B & 400 & 0 & 3.0061 & 8.0363 & 11.0424 & 9.4312 & 1.3512 & 2.35 \\
\hline TETRALIN A & 400 & 0 & 3.0085 & 0 & 3.0085 & 2.495 & 0.8525 & -11.27 \\
\hline TETRALIN B & 400 & 0 & 3.0086 & 0 & 3.0086 & 2.6551 & 0.7308 & -12.54 \\
\hline
\end{tabular}

- - For Tetralin Samples, Mass Balance calculated using only coal material, as all spent tetralin out was removed with the THF.

- N/A indicates data not available 
Table A2 - Mass Balances for Parametric Studies (cont.)

\begin{tabular}{|c|c|c|c|c|c|c|c|c|}
\hline Run Name & $\mathrm{T}\left({ }^{\circ} \mathrm{C}\right)$ & $\begin{array}{c}\mathbf{P} \\
\text { (psig) }\end{array}$ & $\begin{array}{c}\text { Wt Coal } \\
\text { In (g) }\end{array}$ & $\begin{array}{c}\text { Wt } \\
\text { Solvent } \\
\text { In (g) }\end{array}$ & $\begin{array}{l}\text { Total In } \\
\text { (g) }\end{array}$ & $\begin{array}{c}\text { THF } \\
\text { Soluble } \\
\text { Digest (g) }\end{array}$ & $\begin{array}{c}\text { THF } \\
\text { Insoluble } \\
\text { Digest (g) }\end{array}$ & $\begin{array}{c}\text { In-Out } I \\
\text { In (\%) }\end{array}$ \\
\hline$\overline{\mathrm{CBB} A}$ & 400 & 500 & 3.0071 & 8.0042 & 11.0113 & 9.4114 & 1.4887 & 1.01 \\
\hline CBB B & 400 & 500 & 3.0030 & 7.9986 & 11.0016 & 9.4753 & 1.4635 & 0.57 \\
\hline HCBB-L1 A & 400 & 500 & 3.0024 & 7.9937 & 10.9961 & 9.7585 & 1.3287 & -0.83 \\
\hline HCBB-L1 B & 400 & 500 & 3.0062 & 7.9966 & 11.0028 & 9.7552 & 1.3741 & -1.15 \\
\hline HCBB-L2 A & $400^{-1}$ & 500 & 3.0050 & 8.0095 & 11.0145 & 9.8710 & 1.1761 & -0.30 \\
\hline HCBB-L2 B & $400^{\circ}$ & 500 & 3.0008 & 8.0513 & 11.0521 & 9.8404 & 1.1130 & 0.89 \\
\hline HCBB-L3 A & 400 & 500 & 3.0057 & 8.0359 & 11.0416 & 9.8906 & 1.0472 & 0.94 \\
\hline HCBB-L3 B & 400 & 500 & 3.0058 & 8.0721 & 11.0779 & 10.0575 & 1.0085 & 0.11 \\
\hline CBB/HCBB-L2 A & $400^{-1}$ & 500 & 3.0047 & 8.0679 & 11.0726 & 9.7177 & 1.1897 & 1.49 \\
\hline CBB/HCBB-L2 B & $400^{-}$ & 500 & 3.0098 & 8.0410 & 11.0508 & 9.8420 & 1.1144 & 0.85 \\
\hline AO A & 400 & 500 & 3.0104 & 8.0678 & 11.0782 & 8.1396 & 1.9353 & 9.06 \\
\hline AO B & $400^{-}$ & 500 & 3.0082 & 8.0196 & 11.0278 & 7.9887 & 2.0323 & 9.13 \\
\hline MO A & 400 & 500 & 3.0066 & 8.0258 & 11.0324 & $\mathrm{~N} / \mathrm{A}$ & 1.6787 & $\mathrm{~N} / \mathrm{A}$ \\
\hline MO B & $400^{-}$ & 500 & 3.0013 & 8.0368 & 11.0381 & $\bar{N} / \bar{A}$ & 1.6680 & $\mathrm{~N} / \mathrm{A}$ \\
\hline SO A & 400 & 500 & 3.0075 & 8.0190 & 11.0265 & $\mathrm{~N} / \mathrm{A}$ & 1.4177 & $\bar{N} / \bar{A}$ \\
\hline SO B & 400 & 500 & 3.0073 & 8.0197 & 11.0270 & $\mathrm{~N} / \mathrm{A}$ & 1.3730 & $\mathrm{~N} / \mathrm{A}$ \\
\hline HSO-L3 A & 400 & 500 & 3.0085 & 8.0164 & 11.0249 & 9.3809 & 1.2205 & 3.84 \\
\hline HSO-L3 B & 400 & 500 & 3.0017 & 8.0233 & 11.0250 & 9.5244 & 1.1841 & 2.87 \\
\hline SO/HCBB-L2 A & $400^{-}$ & 500 & 3.0035 & 8.1078 & 11.1113 & 9.4060 & 1.7037 & 0.01 \\
\hline SO/HCBB-L2 B & 400 & 500 & 3.0089 & 8.0561 & 11.0650 & 9.5509 & 1.2720 & 2.19 \\
\hline HCBB-L3/HSO-L3 A & $400^{-}$ & 500 & 3.0782 & 8.2198 & 11.2980 & 9.5113 & 1.4659 & 2.84 \\
\hline HCBB-L3/HSO-L3 B & $400^{-}$ & 500 & 3.0197 & 8.0904 & 11.1101 & 9.3220 & 1.4259 & 3.26 \\
\hline TETRALIN A & $400^{-1}$ & 500 & 3.0059 & 0 & 3.0059 & 3.7019 & 0.7164 & -46.99 \\
\hline TETRALIN B & 400 & 500 & 3.0033 & $\overline{0}$ & 3.0033 & 3.4033 & 0.7955 & -39.81 \\
\hline CBB A & 350 & 0 & 3.0009 & 8.1339 & 11.1348 & 8.7820 & 2.1683 & 1.66 \\
\hline CBB B & 350 & 0 & 3.0076 & 8.0473 & 11.0549 & 8.8640 & 2.0838 & 0.97 \\
\hline HCBB-L1 A & 350 & $\overline{0}$ & 3.0129 & 8.0054 & 11.0183 & 8.5655 & 2.4466 & 0.06 \\
\hline HCBB-L1 B & 350 & 0 & 3.0059 & 8.0007 & 11.0066 & 8.6043 & 2.3112 & 0.83 \\
\hline HCBB-L2 A & 350 & 0 & 3.0218 & 8.0186 & 11.0404 & 8.6734 & 2.2089 & 1.43 \\
\hline HCBB-L2 B & 350 & 0 & 3.0122 & 8.0740 & 11.0862 & 8.8502 & 2.1867 & 0.44 \\
\hline HCBB-L3 A & 350 & 0 & 3.0036 & 8.1312 & 11.1348 & 8.9092 & 2.2123 & 0.12 \\
\hline HCBB-L3 B & 350 & 0 & 3.0074 & 8.0504 & 11.0578 & 6.8922 & 2.1250 & 18.45 \\
\hline CBB/HCBB-L2 A & 350 & 0 & 3.0043 & 8.0356 & 11.0399 & 8.6477 & 2.1849 & 1.88 \\
\hline CBB/HCBB-L2 B & 350 & 0 & 3.0025 & 8.0900 & 11.0925 & 8.8385 & 2.1159 & 1.24 \\
\hline SO A & 350 & 0 & 3.0028 & 8.0187 & 11.0215 & 8.9189 & 2.1035 & -0.01 \\
\hline SO B & 350 & 0 & 3.0019 & 8.0430 & 11.0449 & 8.9747 & 2.0614 & 0.08 \\
\hline HSO-L3 A & 350 & 0 & 3.0101 & 8.0136 & 11.0237 & 8.8472 & 1.9257 & 2.28 \\
\hline HSO-L3 B & 350 & 0 & 3.0030 & 8.1314 & 11.1344 & 9.0745 & 1.9056 & 1.39 \\
\hline HCBB-L3/HSO-L3 A & 350 & 0 & 3.0078 & 8.0568 & 11.0646 & 9.0264 & 2.0009 & 0.34 \\
\hline HCBB-L3/HSO-L3 B & 350 & 0 & 3.0048 & 8.0357 & 11.0405 & 8.5683 & 1.9174 & 5.03 \\
\hline TETRALIN A & 350 & 0 & 3.0032 & 0 & 3.0032 & 1.0797 & 1.8442 & 2.64 \\
\hline TETRALIN B & 350 & 0 & 3.0042 & 0 & 3.0042 & 1.0181 & 1.9784 & 0.26 \\
\hline
\end{tabular}


Table A2 - Mass Balances for Parametric Studies (cont.)

\begin{tabular}{|c|c|c|c|c|c|c|c|c|}
\hline Run Name & $\mathrm{T}\left({ }^{\circ} \mathrm{C}\right)$ & $P$ (psig) & $\begin{array}{c}\text { Wt Coal } \\
\text { In (g) }\end{array}$ & $\begin{array}{c}\text { Wt } \\
\text { Solvent In } \\
\text { (g) }\end{array}$ & $\begin{array}{l}\text { Total In } \\
\text { (g) }\end{array}$ & $\begin{array}{c}\text { THF } \\
\text { Soluble } \\
\text { Digest (g) }\end{array}$ & $\begin{array}{c}\text { THF } \\
\text { Insoluble } \\
\text { Digest (g) }\end{array}$ & $\begin{array}{c}\text { In-Out / In } \\
(\%)\end{array}$ \\
\hline CBB A & 350 & 500 & 3.0007 & 8.2188 & 11.2195 & 9.0887 & 2.1000 & 0.27 \\
\hline CBB B & 350 & 500 & 3.0023 & 8.0193 & 11.0216 & 8.8546 & 2.0733 & 0.85 \\
\hline HCBB-L1 A & 350 & 500 & 3.0138 & 8.0580 & 11.0718 & 8.4942 & 2.2835 & 2.66 \\
\hline HCBB-L1 B & 350 & 500 & 3.0055 & 8.0396 & 11.0451 & 8.7105 & 2.2460 & 0.80 \\
\hline HCBB-L2 A & 350 & 500 & 3.0103 & 8.0267 & 11.0370 & 8.5658 & 2.1810 & 2.63 \\
\hline HCBB-L2 B & 350 & 500 & 3.0002 & 8.0853 & 11.0855 & 8.9528 & 1.9895 & 1.29 \\
\hline HCBB-L3 A & 350 & 500 & 3.0052 & 8.0560 & 11.0612 & 8.8289 & 2.2882 & -0.51 \\
\hline HCBB-L3 B & 350 & 500 & 3.0023 & 8.0157 & 11.0180 & 8.9443 & 2.0219 & 0.47 \\
\hline CBB/HCBB-L2 A & 350 & 500 & 3.0029 & 8.0770 & 11.0799 & 8.8913 & 2.0548 & 1.21 \\
\hline CBB/HCBB-L2 B & 350 & 500 & 3.0197 & 8.1246 & 11.1443 & 9.0494 & 2.1022 & -0.07 \\
\hline SO A & 350 & 500 & 3.0022 & 8.0468 & 11.0490 & 8.7363 & 2.2723 & 0.37 \\
\hline SO B & 350 & 500 & 3.0254 & 8.0038 & 11.0292 & 8.9181 & 2.2382 & -1.15 \\
\hline HSO-L3 A & 350 & $500^{-}$ & 3.0062 & 8.0142 & 11.0204 & 8.9044 & 1.9448 & 1.55 \\
\hline HSO-L3 B & 350 & 500 & 3.0026 & 8.1698 & 11.1724 & 8.8684 & 2.0346 & 2.41 \\
\hline HCBB-L3/HSO-L3 A & 350 & 500 & 3.0032 & 8.0355 & 11.0387 & 9.1775 & 1.8711 & -0.09 \\
\hline HCBB-L3/HSO-L3 B & 350 & 500 & 3.0013 & 8.0388 & 11.0401 & 9.0975 & 1.9742 & -0.29 \\
\hline TETRALIN A & 350 & 500 & 3.0027 & 0 & 3.0027 & 1.0353 & 1.9601 & 0.24 \\
\hline TETRALIN B & 350 & 500 & 3.0047 & 0 & 3.0047 & 1.0458 & 2.0308 & -2.39 \\
\hline CBB A & 450 & 0 & 3.0003 & 8.0014 & 11.0017 & 8.961 & 1.5256 & 4.68 \\
\hline CBB B & 450 & 0 & 3.0076 & 8.0257 & 11.0333 & 9.0252 & 1.6906 & 2.88 \\
\hline HCBB-L1 A & 450 & 0 & 3.0044 & 8.0536 & 11.058 & 9.094 & 1.6072 & 3.23 \\
\hline HCBB-L1 B & 450 & 0 & 3.0080 & 8.0623 & 11.0703 & 9.0306 & 1.5689 & 4.25 \\
\hline HCBB-L2 A & 450 & 0 & 3.0011 & 8.0145 & 11.0156 & 9.2831 & 1.4528 & 2.54 \\
\hline HCBB-L2 B & 450 & 0 & 3.0094 & 8.0088 & 11.0182 & 9.0974 & 1.5200 & 3.64 \\
\hline HCBB-L3 A & 450 & 0 & 3.0087 & 8.0365 & 11.0452 & 8.9404 & 1.6973 & 3.69 \\
\hline HCBB-L3 B & 450 & 0 & 3.0012 & 8.0745 & 11.0757 & 9.1919 & 1.4196 & 4.19 \\
\hline CBB/HCBB-L2 A & 450 & 0 & 3.0022 & 8.0252 & 11.0274 & 8.9699 & 1.6792 & 3.43 \\
\hline CBB/HCBB-L2 B & 450 & 0 & 3.0022 & 8.0706 & 11.0728 & 8.7763 & 1.8812 & 3.75 \\
\hline SO A & 450 & 0 & 3.0130 & 8.0352 & 11.0482 & 5.6835 & 2.2750 & 27.97 \\
\hline SO B & 450 & 0 & 3.0062 & 8.0227 & 11.0289 & 5.8242 & 2.9513 & 20.43 \\
\hline HSO-L3 A & 450 & 0 & 3.0015 & 8.0200 & 11.0215 & 6.5997 & 2.5373 & 17.10 \\
\hline HSO-L3 B & 450 & 0 & 3.0093 & 8.0216 & 11.0309 & 6.2980 & 2.6301 & 19.06 \\
\hline HCBB-L3/HSO-L3 A & 450 & 0 & 3.0006 & 8.0786 & 11.0792 & 8.1156 & 1.8978 & 9.62 \\
\hline HCBB-L3/HSO-L3 B & 450 & 0 & 3.0086 & 8.0363 & 11.0449 & 8.0970 & 1.8394 & 10.04 \\
\hline TETRALIN A & 450 & 0 & 3.0070 & 0 & 3.0070 & 1.8955 & 0.5418 & 18.95 \\
\hline TETRALIN B & 450 & 0 & 3.0027 & 0 & 3.0027 & 2.0517 & 0.6789 & 9.06 \\
\hline
\end{tabular}


Table A2 - Mass Balances for Parametric Studies (cont.)

\begin{tabular}{|c|c|c|c|c|c|c|c|c|}
\hline Run Name & $\mathrm{T}\left({ }^{\circ} \mathrm{C}\right)$ & $\begin{array}{c}P \\
\text { (psig) }\end{array}$ & $\begin{array}{c}\text { Wt Coal } \\
\text { In (g) }\end{array}$ & $\begin{array}{c}\text { Wt } \\
\text { Solvent In } \\
\text { (g) }\end{array}$ & $\begin{array}{c}\text { Total In } \\
\text { (g) }\end{array}$ & $\begin{array}{c}\text { THF } \\
\text { Soluble } \\
\text { Digest (g) }\end{array}$ & $\begin{array}{c}\text { THF } \\
\text { Insoluble } \\
\text { Digest (g) }\end{array}$ & $\begin{array}{c}\text { In-Out I } \\
\text { In (\%) }\end{array}$ \\
\hline$\overline{\mathrm{CBB} A}$ & 450 & 500 & 3.0087 & 8.0248 & 11.0335 & 9.0102 & 1.5908 & 3.92 \\
\hline CBB B & 450 & 500 & 3.0041 & 8.0257 & 11.0298 & 8.8009 & 1.7382 & 4.45 \\
\hline HCBB-L1 A & 450 & 500 & 3.0024 & 8.0781 & 11.0785 & 9.1984 & 1.4568 & 3.82 \\
\hline HCBB-L1 B & 450 & 500 & 3.015 & 8.0046 & 11.0196 & 9.1575 & 1.4582 & 3.67 \\
\hline HCBB-L2 A & 450 & 500 & 3.002 & 8.0127 & 11.0147 & 8.6899 & 1.5367 & 7.15 \\
\hline HCBB-L2 B & 450 & 500 & 3.0019 & 8.0437 & 11.0456 & 7.4654 & 1.8791 & 15.40 \\
\hline HCBB-L3 A & 450 & 500 & 3.0024 & 8.0267 & 11.0291 & 9.0637 & 1.4345 & 4.81 \\
\hline HCBB-L3 B & 450 & 500 & 3.0035 & 8.0346 & 11.0381 & 9.1511 & 1.3436 & 4.92 \\
\hline CBB/HCBB-L2 A & 450 & 500 & 3.0033 & 8.0250 & 11.0283 & 8.9014 & 1.6674 & 4.17 \\
\hline CBB/HCBB-L2 B & 450 & 500 & 3.0075 & 8.2651 & 11.2726 & 9.0723 & 1.6232 & 5.12 \\
\hline SO A & 450 & 500 & 3.0036 & 8.0197 & 11.0233 & 6.9829 & 2.9697 & 9.71 \\
\hline SO B & 450 & 500 & 3.0167 & $8.0380^{-}$ & 11.0547 & 6.6624 & 3.0119 & 12.49 \\
\hline HSO-L3 A & 450 & 500 & 3.0036 & 8.0115 & 11.0151 & 6.7525 & 2.6233 & 14.88 \\
\hline HSO-L3 B & 450 & 500 & 3.0029 & 8.1072 & 11.1101 & 6.9329 & 2.5608 & 14.55 \\
\hline HCBB-L3/HSO-L3 A & 450 & 500 & 3.0077 & 8.0799 & 11.0876 & 7.9507 & 1.8512 & 11.60 \\
\hline HCBB-L3/HSO-L3 B & 450 & 500 & 3.0074 & 8.2185 & 11.2259 & 8.3499 & 1.9421 & 8.32 \\
\hline TETRALIN A & 450 & 500 & 3.0026 & 0 & 3.0026 & 3.0277 & 0.5880 & -20.42 \\
\hline TETRALIN B & 450 & 500 & 3.0022 & 0 & 3.0022 & 1.9400 & 0.5966 & 15.51 \\
\hline
\end{tabular}

Table A3 - Mass Balances for One-Gallon Scale-Up Digestions

\begin{tabular}{|c|c|c|c|c|c|c|c|c|}
\hline $\begin{array}{c}\text { Sample } \\
\text { Name }\end{array}$ & Solvent Used & $\begin{array}{c}\text { Mass } \\
\text { Solvent } \\
\text { (g) }\end{array}$ & $\begin{array}{c}\text { Mass } \\
\text { Coal }(\mathrm{g})\end{array}$ & $\begin{array}{l}\text { Mass } \\
\text { Product } \\
(\mathrm{g})\end{array}$ & $\begin{array}{l}\text { THF Soluble } \\
\text { Residue (g) }\end{array}$ & $\begin{array}{c}\text { THF } \\
\text { Insoluble } \\
\text { Residue (g) }\end{array}$ & $\begin{array}{c}\text { Mass } \\
\text { Off-Gas } \\
\text { (g) }\end{array}$ & $\begin{array}{c}\text { In-Out I } \\
\text { In (\%) }\end{array}$ \\
\hline A086 & HCBB-L3 & 1495.94 & 600.18 & 1783.08 & 87.52 & 73.34 & 0 & 7.26 \\
\hline A090 & $\begin{array}{l}50 \% \text { HCBB-L3 } \\
\& 50 \% \text { HSO-L3 }\end{array}$ & 1530.86 & 600.38 & 1660.93 & 219.58 & 156.09 & 14.36 & 3.77 \\
\hline A095 & HSO-L3 & 1520.58 & 600.26 & 1088.18 & 515.36 & 322.53 & 10.38 & 8.69 \\
\hline A098 & HCBB-L3 & 1500.06 & 603.46 & 1787.57 & 138.64 & 87.39 & 6.38 & 3.97 \\
\hline A100 & HMO-L3 & 1509.38 & 600.15 & 1397.23 & 349.72 & 272.45 & 12.77 & 3.67 \\
\hline B003 & $\begin{array}{l}50 \% \text { HCBB-L3 } \\
\& 50 \% \text { HMO-L3 }\end{array}$ & 1537.26 & 600.31 & 1799.32 & 155.81 & 121.95 & 12.76 & 2.23 \\
\hline
\end{tabular}


Table A4 - Mass Balances for Air-Blowing Studies

\begin{tabular}{|c|c|c|c|c|}
\hline $\begin{array}{c}\text { Sample } \\
\text { Name }\end{array}$ & $\begin{array}{c}\text { Mass } \\
\text { Digest In } \\
(\mathbf{g})\end{array}$ & $\begin{array}{c}\text { Mass } \\
\text { Product } \\
(\mathbf{g})\end{array}$ & $\begin{array}{c}\text { Mass } \\
\text { Distillate } \\
(\mathbf{g})\end{array}$ & $\begin{array}{c}\text { In - Out I } \\
\text { In (\%) }\end{array}$ \\
\hline $\mathrm{A086}$ & 304.6 & 256.86 & 11.09 & 12.03 \\
\hline $\mathrm{A} 090$ & 306.02 & 249.12 & 12.93 & 14.37 \\
\hline $\mathrm{A095}$ & 297.04 & 247.37 & 16.12 & 11.29478 \\
\hline $\mathrm{A098}$ & 303.73 & 259.92 & 17.61 & 8.626082 \\
\hline $\mathrm{A} 100$ & 320.56 & 192.68 & 85.45 & 13.23621 \\
\hline $\mathrm{B003}$ & 307.01 & 264.58 & 14.71 & 9.029022 \\
\hline
\end{tabular}

Table A5 - Mass Balances for Vacuum Distillation of Digests

\begin{tabular}{|r|c|c|c|c|c|c|}
\hline & A086 & A090 & A095 & A098 & A100 & B003 \\
\hline Sample Wt. (g) & 670.78 & 514.10 & 521.52 & 510.91 & 512.38 & 516.69 \\
\hline Max Temp. ( ${ }^{\circ}$ C) & 195 & 260 & 275 & 235 & 231 & 225 \\
\hline Bottoms Wt. (g) & 320.05 & 345.40 & 296.15 & 212.04 & 141.29 & 235.08 \\
\hline Distillate Wt. (g) & 350.73 & 279.88 & 252.85 & 292.37 & 277.00 & 268.38 \\
\hline In-Out / In (\%) & 0.00 & -21.63 & -5.27 & 1.27 & 18.36 & 2.56 \\
\hline
\end{tabular}


APPENDIX B: $\quad$ ANALYTICAL DATA TABLES 
Table B1 - CBB Level 1 Hydrogenation P \& T Data

\begin{tabular}{|cccc|}
\hline $\begin{array}{c}\text { Time } \\
\text { (min) }\end{array}$ & $\begin{array}{c}\text { Furnace } \\
\mathbf{T}\left({ }^{\circ} \mathbf{C}\right)\end{array}$ & $\begin{array}{c}\text { Reactor } \\
\left({ }^{\circ} \mathbf{C}\right)\end{array}$ & $\begin{array}{c}\text { Reactor P } \\
\text { (psig) }\end{array}$ \\
\hline 0 & 200 & 23 & 500 \\
10 & 200 & 68 & \\
20 & 200 & 166 & \\
25 & 200 & 214 & 720 \\
30 & 250 & 229 & 760 \\
40 & 250 & 256 & 750 \\
$\mathbf{4 5}$ & 250 & $\mathbf{2 7 5}$ & $\mathbf{7 5 0}$ \\
50 & 250 & 275 & 700 \\
55 & 250 & 288 & 640 \\
65 & 250 & 276 & 580 \\
75 & 250 & 273 & 550 \\
85 & 250 & 275 & 510 \\
95 & 250 & 276 & 480 \\
$\mathbf{1 0 5}$ & $\mathbf{2 2 0}$ & $\mathbf{2 7 5}$ & $\mathbf{4 8 0}$ \\
Next Day & 32 & 200 \\
\hline \multicolumn{4}{|c}{} \\
\hline
\end{tabular}

Table B2 - CBB Level 2 Hydrogenation P \& T Data

\begin{tabular}{|c|c|c|c|}
\hline $\begin{array}{l}\text { Tíme } \\
\text { (min) }\end{array}$ & $\begin{array}{c}\text { Furnace } \\
\left.\text { T ( }{ }^{\circ} \mathrm{C}\right)\end{array}$ & $\begin{array}{c}\text { Reactor } \\
\left({ }^{\circ} \mathrm{C}\right)\end{array}$ & $\begin{array}{l}\text { Reactor P } \\
\text { (psig) }\end{array}$ \\
\hline 0 & 250 & 38 & 480 \\
\hline 15 & 250 & 151 & 600 \\
\hline 30 & 250 & 255 & 740 \\
\hline 32 & 300 & 270 & 760 \\
\hline 45 & 350 & 336 & 580 \\
\hline 50 & 325 & 354 & 480 \\
\hline 55 & 325 & 361 & 440 \\
\hline 60 & 320 & 355 & 400 \\
\hline 65 & 320 & 349 & 380 \\
\hline 75 & 320 & 358 & 350 \\
\hline 90 & 320 & 357 & 320 \\
\hline 100 & 320 & 352 & 300 \\
\hline 110 & 320 & 352 & 300 \\
\hline \multicolumn{2}{|c|}{ Next Day } & 39 & 80 \\
\hline
\end{tabular}


Table B3 - CBB Level 3 Hydrogenation P\&T Data

\begin{tabular}{|cccc|}
\hline $\begin{array}{c}\text { Time } \\
(\mathbf{m i n})\end{array}$ & $\begin{array}{c}\text { Furnace } \\
\mathbf{T}\left({ }^{\circ} \mathbf{C}\right)\end{array}$ & $\begin{array}{c}\text { Reactor T } \\
\left({ }^{\circ} \mathbf{C}\right)\end{array}$ & $\begin{array}{c}\text { Reactor } \mathbf{P} \\
(\mathbf{p s i g})\end{array}$ \\
\hline 0 & 400 & 25 & 750 \\
15 & 400 & 93 & 920 \\
30 & 400 & 209 & 1140 \\
35 & 400 & 252 & 1200 \\
40 & 400 & 295 & 1190 \\
45 & 400 & 326 & 1080 \\
$\mathbf{5 0}$ & $\mathbf{4 0 0}$ & $\mathbf{3 5 7}$ & $\mathbf{8 8 0}$ \\
53 & 375 & 372 & 750 \\
55 & 375 & 381 & 680 \\
65 & 375 & 372 & 540 \\
85 & 375 & 375 & 480 \\
100 & 375 & 375 & 460 \\
105 & 375 & 375 & 450 \\
$\mathbf{1 1 0}$ & $\mathbf{3 7 5}$ & $\mathbf{3 7 5}$ & $\mathbf{4 5 0}$ \\
Next Day & 47 & 80 \\
\hline \multicolumn{4}{|c}{} \\
\hline
\end{tabular}

Table B4 - Slurry Oil Level 3 Hydrogenation P \& T Data

\begin{tabular}{|c|c|c|c|}
\hline $\begin{array}{l}\text { Time } \\
\text { (min) }\end{array}$ & $\begin{array}{c}\text { Furnace } \\
\mathrm{T}\left({ }^{\circ} \mathrm{C}\right)\end{array}$ & $\begin{array}{c}\text { Reactor } \\
\left({ }^{\circ} \mathrm{C}\right)\end{array}$ & $\begin{array}{l}\text { Reactor P } \\
\text { (psig) }\end{array}$ \\
\hline$\overline{0}$ & 375 & 15 & 750 \\
\hline 10 & 375 & 69 & 820 \\
\hline 25 & 375 & 180 & 1080 \\
\hline 40 & 375 & 285 & 1320 \\
\hline 55 & 375 & 361 & 1080 \\
\hline 58 & 375 & 375 & 960 \\
\hline 60 & 375 & 382 & 880 \\
\hline 65 & 375 & 382 & 740 \\
\hline 67 & 375 & 377 & 680 \\
\hline 72 & 375 & 374 & 600 \\
\hline 80 & 375 & 378 & 580 \\
\hline 90 & 375 & 379 & 560 \\
\hline 100 & 375 & 377 & 550 \\
\hline 118 & 375 & 377 & 580 \\
\hline \multicolumn{2}{|c|}{ Next Day } & 35 & 100 \\
\hline
\end{tabular}


Table B5 - Maraflex Oil Level 3 Hydrogenation P \& T Data

\begin{tabular}{|cccc|}
\hline $\begin{array}{c}\text { Time } \\
(\mathbf{m i n})\end{array}$ & $\begin{array}{c}\text { Furnace } \\
\mathbf{T}\left({ }^{\circ} \mathbf{C}\right)\end{array}$ & $\begin{array}{c}\text { Reactor } \\
\left({ }^{\circ} \mathbf{C}\right)\end{array}$ & $\begin{array}{c}\text { Reactor } \mathbf{P} \\
(\mathbf{p s i g})\end{array}$ \\
\hline 0 & 200 & 19 & 750 \\
15 & 200 & 117 & 900 \\
25 & 200 & 200 & 1100 \\
36 & 250 & 247 & 1200 \\
55 & 275 & 275 & 1140 \\
115 & 305 & 300 & 820 \\
120 & 350 & 325 & 800 \\
125 & 375 & 350 & 750 \\
$\mathbf{1 3 5}$ & $\mathbf{3 7 5}$ & $\mathbf{3 7 5}$ & $\mathbf{6 2 0}$ \\
145 & 375 & 380 & 560 \\
150 & 375 & 375 & 500 \\
193 & 375 & 375 & 450 \\
$\mathbf{1 9 5}$ & $\mathbf{3 7 5}$ & $\mathbf{3 7 6}$ & $\mathbf{4 5 0}$ \\
Next Day & 35 & 100 \\
\hline \multicolumn{4}{|c}{}
\end{tabular}

Table B6 - Solvent Ash Analyses

\begin{tabular}{|c|c|c|}
\hline & \multicolumn{2}{|c|}{ \% Ash } \\
\hline Solvent & 1 & 2 \\
\hline CBB & 0.01 & 0 \\
\hdashline HCBB-L1 & 0.02 & 0.04 \\
\hdashline HCBB-L2 & 0.02 & 0.02 \\
HCBB-L3 & 0 & 0 \\
SO & 0.01 & 0.03 \\
HSO-L3 & 0.05 & 0.04 \\
\hdashline MO & 0 & 0 \\
HMO-L3 & 0.01 & 0.01 \\
\hline
\end{tabular}


Table B7 - A086 Digestion P \& T Data

\begin{tabular}{|c|c|c|c|c|c|}
\hline \multirow{2}{*}{$\begin{array}{c}\begin{array}{c}\text { Time } \\
(\mathrm{min})\end{array} \\
0\end{array}$} & \multicolumn{2}{|c|}{$\begin{array}{c}\text { Furnace T: Act } \\
\text { I SP }\left({ }^{\circ} \mathrm{C}\right)\end{array}$} & \multicolumn{2}{|c|}{$\begin{array}{l}\text { Reactor T: } \\
\text { Act / SP }\left({ }^{\circ} \mathrm{C}\right)\end{array}$} & \multirow{2}{*}{$\begin{array}{c}\begin{array}{c}\text { Pressure } \\
\text { (psig) }\end{array} \\
300\end{array}$} \\
\hline & 40 & 500 & 33 & 400 & \\
\hline 17 & 332 & 500 & 57 & 400 & 300 \\
\hline 27 & 414 & 500 & 100 & 400 & 300 \\
\hline 36 & 464 & 500 & 150 & 400 & 300 \\
\hline 46 & 495 & 500 & 200 & 400 & 300 \\
\hline 58 & 494 & 500 & 250 & 400 & 300 \\
\hline 67 & 495 & 590 & 279 & 400 & 300 \\
\hline 75 & 516 & 590 & 300 & 400 & 300 \\
\hline 80 & 558 & 590 & 350 & 400 & 300 \\
\hline 90 & 570 & 550 & 375 & 400 & 300 \\
\hline 93 & 539 & 500 & 385 & 400 & 300 \\
\hline 103 & 498 & 500 & 395 & 400 & 325 \\
\hline 115 & 455 & 500 & 402 & 400 & 400 \\
\hline 140 & 412 & 500 & 400 & 400 & 425 \\
\hline 163 & 407 & 500 & 399 & 400 & 450 \\
\hline \multicolumn{3}{|c|}{ Next Day } & 100 & 100 & 300 \\
\hline
\end{tabular}

Table B8 - A090 Digestion P \& T Data

\begin{tabular}{|c|c|c|c|c|c|}
\hline \multirow{2}{*}{$\begin{array}{c}\begin{array}{c}\text { Time } \\
\text { (min) }\end{array} \\
0\end{array}$} & \multicolumn{2}{|c|}{$\begin{array}{l}\text { Furnace T: } \\
\text { Act / SP }\left({ }^{\circ} \mathrm{C}\right)\end{array}$} & \multicolumn{2}{|c|}{\begin{tabular}{|c} 
Reactor T: Act \\
I SP $\left({ }^{\circ} \mathrm{C}\right)$
\end{tabular}} & \multirow{2}{*}{$\begin{array}{c}\begin{array}{c}\text { Pressure } \\
\text { (psig) }\end{array} \\
300\end{array}$} \\
\hline & 19 & 590 & 18 & 425 & \\
\hline 20 & 272 & 590 & 62 & 425 & 300 \\
\hline 35 & 287 & 590 & 153 & 425 & 300 \\
\hline 46 & 316 & 590 & 200 & 425 & 300 \\
\hline 65 & 368 & 590 & 275 & 425 & 300 \\
\hline 70 & 385 & 590 & 300 & 425 & 300 \\
\hline 85 & 427 & 590 & 360 & 425 & 300 \\
\hline 92 & 439 & 590 & 375 & 425 & 300 \\
\hline 100 & 456 & 590 & 400 & 425 & 325 \\
\hline 106 & 467 & 440 & 415 & 425 & 325 \\
\hline 110 & 447 & 440 & 423 & 425 & 450 \\
\hline 140 & 441 & 440 & 424 & 425 & 675 \\
\hline 155 & 441 & 450 & 423 & 425 & 825 \\
\hline 170 & 444 & 450 & 424 & 425 & 925 \\
\hline \multicolumn{3}{|c|}{ Next Day } & 100 & 100 & 525 \\
\hline
\end{tabular}


Table B9 - A095 Digestion P \& T Data

\begin{tabular}{|c|cc|cc|c|}
\hline $\begin{array}{c}\text { Time } \\
\text { (min) }\end{array}$ & \multicolumn{2}{|c|}{$\begin{array}{c}\text { Furnace T: } \\
\text { Act / SP }\left({ }^{\circ} \mathbf{C}\right)\end{array}$} & $\begin{array}{c}\text { Reactor T: } \\
\text { Act / SP }\left({ }^{\circ} \mathbf{C}\right)\end{array}$ & $\begin{array}{c}\text { Pressure } \\
(\text { psig) }\end{array}$ \\
\hline 0 & 23 & 590 & 25 & 425 & 300 \\
23 & 214 & 590 & 84 & 425 & 300 \\
52 & 315 & 590 & 205 & 425 & 300 \\
65 & 363 & 590 & 275 & 425 & 300 \\
87 & 427 & 590 & 350 & 425 & 300 \\
107 & 470 & 450 & 414 & 425 & 300 \\
109 & 450 & 450 & 421 & 425 & $\mathbf{3 7 5}$ \\
124 & 414 & 450 & 422 & 425 & 375 \\
142 & 414 & 450 & 421 & 425 & 450 \\
154 & 414 & 450 & 426 & 425 & 550 \\
$\mathbf{1 6 9}$ & $\mathbf{4 2 9}$ & $\mathbf{4 5 0}$ & $\mathbf{4 2 8}$ & $\mathbf{4 2 5}$ & $\mathbf{6 2 5}$ \\
& Next Day & & $\mathbf{1 0 0}$ & $\mathbf{1 0 0}$ & \\
\hline
\end{tabular}

Table B10 - A098 Digestion P \& T Data

\begin{tabular}{|c|c|c|c|c|c|}
\hline $\begin{array}{l}\text { Time } \\
(\min )\end{array}$ & \multicolumn{2}{|c|}{$\begin{array}{l}\text { Furnace T: } \\
\text { Act / SP }\left({ }^{\circ} \mathrm{C}\right)\end{array}$} & \multicolumn{2}{|c|}{$\begin{array}{l}\text { Reactor T: } \\
\text { Act / SP }\left({ }^{\circ} \mathrm{C}\right)\end{array}$} & \multirow{2}{*}{$\begin{array}{c}\text { Pressure } \\
\text { (psig) }\end{array}$} \\
\hline 0 & 23 & 590 & 21 & 425 & \\
\hline 30 & 243 & 590 & 116 & 425 & 300 \\
\hline 65 & 368 & 590 & 280 & 425 & 300 \\
\hline 90 & 514 & 590 & 370 & 425 & 300 \\
\hline 105 & 546 & 590 & 400 & 425 & 325 \\
\hline 110 & 553 & 450 & 410 & 425 & 325 \\
\hline 115 & 494 & 475 & 420 & 425 & 400 \\
\hline 140 & 473 & 485 & 422 & 425 & 500 \\
\hline 175 & 493 & 485 & 425 & 425 & 700 \\
\hline Next D & & & 100 & 100 & 400 \\
\hline
\end{tabular}

Table B11 - A100 Digestion P \& T Data

\begin{tabular}{|c|ccccc|}
\hline $\begin{array}{c}\text { Time } \\
\text { (min) }\end{array}$ & $\begin{array}{c}\text { Furnace T: Act } \\
\text { I SP }\left({ }^{\circ} \mathbf{C}\right)\end{array}$ & \multicolumn{2}{|c|}{$\begin{array}{c}\text { Reactor T: } \\
\text { Act } / \text { SP }\left({ }^{\circ} \mathbf{C}\right)\end{array}$} & $\begin{array}{c}\text { Pressure } \\
(\mathbf{p s i g})\end{array}$ \\
\hline 0 & 106 & 590 & 30 & 425 & 300 \\
20 & 237 & 590 & 95 & 425 & 300 \\
35 & 300 & 590 & 166 & 425 & 300 \\
50 & 346 & 590 & 235 & 425 & 300 \\
102 & 473 & 460 & 415 & 425 & 400 \\
104 & 466 & 460 & $\mathbf{4 2 0}$ & $\mathbf{4 2 5}$ & $\mathbf{4 0 0}$ \\
128 & 457 & 460 & 423 & 425 & 650 \\
155 & 406 & 460 & 428 & 425 & 850 \\
$\mathbf{1 6 4}$ & $\mathbf{4 1 9}$ & $\mathbf{4 6 0}$ & $\mathbf{4 2 2}$ & $\mathbf{4 2 5}$ & $\mathbf{8 5 0}$ \\
\multicolumn{3}{|c|}{ Next Day } & $\mathbf{1 0 0}$ & $\mathbf{1 0 0}$ & $\mathbf{5 0 0}$ \\
\hline
\end{tabular}


Table B12 - B003 Digestion P \& T Data

\begin{tabular}{|c|ccccc|}
\hline $\begin{array}{c}\text { Time } \\
(\mathbf{m i n})\end{array}$ & $\begin{array}{c}\text { Furnace T: Act } \\
\text { I SP }\left({ }^{\circ} \mathbf{C}\right)\end{array}$ & \multicolumn{2}{|c|}{$\begin{array}{c}\text { Reactor T: Act } \\
\text { I SP }\left({ }^{\circ} \mathbf{C}\right)\end{array}$} & $\begin{array}{c}\text { Pressure } \\
\text { (psig) }\end{array}$ \\
\hline 0 & 23 & 590 & 23 & 425 & 300 \\
33 & 131 & 590 & 160 & 425 & 300 \\
43 & 302 & 590 & 177 & 425 & 300 \\
$\mathbf{1 1 0}$ & $\mathbf{4 8 2}$ & $\mathbf{4 6 0}$ & $\mathbf{4 2 1}$ & $\mathbf{4 2 5}$ & $\mathbf{4 0 0}$ \\
115 & 436 & 460 & 431 & 425 & 500 \\
133 & 453 & 460 & 422 & 425 & 600 \\
148 & 429 & 450 & 422 & 425 & 700 \\
163 & 413 & 450 & 423 & 425 & 750 \\
$\mathbf{1 7 0}$ & $\mathbf{4 5 0}$ & $\mathbf{4 5 0}$ & $\mathbf{4 2 2}$ & $\mathbf{4 2 5}$ & $\mathbf{8 0 0}$ \\
\multicolumn{7}{c}{ Next Day } & & $\mathbf{1 0 0}$ & $\mathbf{1 0 0}$ & $\mathbf{5 0 0}$ \\
\hline
\end{tabular}

Table B13 - Coal Digest Analysis Data

\begin{tabular}{|c|c|c|c|c|c|c|c|c|c|}
\hline \multirow[b]{2}{*}{$\begin{array}{l}\text { Sample } \\
\text { Name }\end{array}$} & \multirow[b]{2}{*}{$\begin{array}{c}\text { Conversion } \\
\text { (\%daf) }\end{array}$} & \multicolumn{2}{|c|}{$\%$ Ash } & \multicolumn{2}{|c|}{$\begin{array}{c}\text { Conradson } \\
\text { Carbon Yield } \\
(\%)\end{array}$} & \multicolumn{2}{|c|}{$\begin{array}{l}\text { WVU Coke } \\
\text { Yield (\%) }\end{array}$} & \multicolumn{2}{|c|}{$\begin{array}{c}\text { Toluene } \\
\text { Insolubles } \\
(\%)\end{array}$} \\
\hline & & 1 & 2 & 1 & 2 & 1 & 2 & 1 & 2 \\
\hline A086 & 96.38 & 0.91 & 0.96 & 20.86 & 20.33 & 23.53 & 23.88 & 18.83 & 19.05 \\
\hline A090 & 81.25 & 0.35 & 0.34 & 24.87 & 25.04 & 28.30 & 28.25 & 16.56 & 20.60 \\
\hline A095 & 50.80 & 0.04 & 0.07 & 27.14 & 27.06 & 29.88 & 28.79 & 18.19 & 17.32 \\
\hline A098 & 93.89 & 1.09 & 1.08 & 22.60 & 22.65 & 26.17 & 25.03 & 20.36 & 20.29 \\
\hline A100 & 59.95 & 0.00 & 0.00 & 13.98 & 14.95 & 16.81 & 13.97 & 10.63 & 10.58 \\
\hline B003 & 87.49 & 0.50 & 0.44 & 20.55 & 20.35 & 22.24 & 22.01 & 17.33 & 17.79 \\
\hline
\end{tabular}

Table B14 - AB-A086 T Data

\begin{tabular}{|c|cc|cc|}
\hline $\begin{array}{c}\text { TIme } \\
\text { (min) }\end{array}$ & \multicolumn{2}{|c|}{$\begin{array}{c}\text { Furnace T: Act } \\
\text { I SP }\left({ }^{\circ} \mathbf{C}\right)\end{array}$} & \multicolumn{2}{c|}{$\begin{array}{c}\text { Process T: } \\
\text { Act } / \text { SP }\left({ }^{\circ} \mathbf{C}\right)\end{array}$} \\
\hline 0 & 268 & 350 & 50 & 250 \\
15 & 345 & 350 & 150 & 250 \\
21 & 348 & 300 & 200 & 250 \\
27 & 322 & 300 & 225 & 250 \\
30 & 307 & 300 & 235 & 250 \\
$\mathbf{3 5}$ & $\mathbf{3 0 5}$ & $\mathbf{3 0 0}$ & $\mathbf{2 4 5}$ & $\mathbf{2 5 0}$ \\
40 & 287 & 290 & 258 & 250 \\
65 & 302 & 270 & 255 & 250 \\
90 & 286 & 265 & 253 & 250 \\
105 & 225 & 255 & 255 & 250 \\
120 & 254 & 245 & 254 & 250 \\
215 & 221 & 245 & 251 & 250 \\
$\mathbf{3 3 5}$ & $\mathbf{2 5 5}$ & $\mathbf{2 4 5}$ & $\mathbf{2 5 0}$ & $\mathbf{2 5 0}$ \\
\hline
\end{tabular}

Table B15 - AB-A090 T Data

\begin{tabular}{|c|cc|cc|}
\hline $\begin{array}{c}\text { TIme } \\
\text { (min) }\end{array}$ & \multicolumn{2}{|c|}{$\begin{array}{c}\text { Furnace T: } \\
\text { Act / SP }\left({ }^{\circ} \mathbf{C}\right)\end{array}$} & $\begin{array}{c}\text { Process T: Act } \\
\text { I SP }\left({ }^{\circ} \mathbf{C}\right)\end{array}$ \\
\hline 0 & 250 & 350 & 50 & 350 \\
15 & 344 & 350 & 162 & 250 \\
19 & 345 & 300 & 200 & 250 \\
30 & 306 & 275 & 236 & 250 \\
35 & 272 & 260 & 245 & $\mathbf{2 5 0}$ \\
42 & 268 & 260 & 250 & 250 \\
46 & 246 & 260 & 251 & 250 \\
52 & 234 & 260 & 248 & 250 \\
101 & 224 & 260 & 248 & 250 \\
116 & 226 & 260 & 253 & 250 \\
224 & 207 & 260 & 249 & 250 \\
239 & 227 & 260 & 254 & 250 \\
289 & 211 & 260 & 248 & 250 \\
$\mathbf{3 6 5}$ & $\mathbf{2 4 9}$ & $\mathbf{2 6 0}$ & $\mathbf{2 5 4}$ & $\mathbf{2 5 0}$ \\
\hline
\end{tabular}


Table B16 - AB-A095 T Data

\begin{tabular}{|c|cccc|}
\hline $\begin{array}{c}\text { TIme } \\
(\mathbf{m i n})\end{array}$ & $\begin{array}{c}\text { Furnace T: Act } \\
\text { I SP }\left({ }^{\circ} \mathbf{C}\right)\end{array}$ & \multicolumn{2}{c|}{$\begin{array}{c}\text { Process T: } \\
\text { Act } / \text { SP }\left({ }^{\circ} \mathbf{C}\right)\end{array}$} \\
\hline 0 & 344 & 350 & 70 & 350 \\
9 & 349 & 300 & 179 & 250 \\
24 & 305 & 275 & 235 & 250 \\
$\mathbf{3 0}$ & $\mathbf{2 8 3}$ & $\mathbf{2 6 0}$ & $\mathbf{2 4 5}$ & $\mathbf{2 5 0}$ \\
34 & 263 & 260 & 248 & 250 \\
59 & 226 & 260 & 251 & 250 \\
87 & 264 & 260 & 253 & 250 \\
139 & 231 & 260 & 248 & 250 \\
207 & 246 & 260 & 255 & 250 \\
260 & 210 & 260 & 250 & 250 \\
$\mathbf{3 3 0}$ & $\mathbf{2 5 5}$ & $\mathbf{2 6 0}$ & $\mathbf{2 5 1}$ & $\mathbf{2 5 0}$ \\
\hline
\end{tabular}

Table B18 - AB-A100 Data

\begin{tabular}{|c|cc|cc|}
\hline $\begin{array}{c}\text { Time } \\
(\mathbf{m i n})\end{array}$ & \multicolumn{2}{|c|}{$\begin{array}{c}\text { Furnace T: } \\
\text { Act } / \text { SP }\left({ }^{\circ} \mathbf{C}\right)\end{array}$} & \multicolumn{2}{c|}{ Process T: } \\
Act & SP $\left({ }^{\circ} \mathbf{C}\right)$ \\
\hline 0 & 86 & 350 & 21 & 250 \\
20 & 350 & 350 & 138 & 250 \\
26 & 353 & 350 & 188 & 250 \\
34 & 346 & 250 & 220 & 250 \\
49 & 249 & 260 & 242 & 250 \\
57 & 269 & 250 & 245 & 250 \\
136 & 229 & 250 & 249 & 250 \\
184 & 264 & 250 & 251 & 250 \\
357 & $\mathbf{2 4 4}$ & $\mathbf{2 5 0}$ & $\mathbf{2 5 1}$ & $\mathbf{2 5 0}$ \\
\hline
\end{tabular}

Table B17 - AB-A098 T Data

\begin{tabular}{|c|cc|cc|}
\hline $\begin{array}{c}\text { Time } \\
\text { (min) }\end{array}$ & \multicolumn{2}{|c|}{$\begin{array}{c}\text { Furnace T: } \\
\text { Act } / \text { SP }\left({ }^{\circ} \mathbf{C}\right)\end{array}$} & \multicolumn{2}{c|}{$\begin{array}{c}\text { Process } / \text { S } \\
\text { Act }\left({ }^{\circ} \mathbf{C}\right)\end{array}$} \\
\hline 0 & 28 & 350 & 23 & 250 \\
6 & 191 & 350 & 41 & 250 \\
30 & 345 & 250 & 198 & 250 \\
45 & 250 & 260 & 229 & 250 \\
61 & 282 & 250 & 243 & 250 \\
63 & $\mathbf{2 4 5}$ & $\mathbf{2 5 0}$ & $\mathbf{2 4 5}$ & $\mathbf{2 5 0}$ \\
96 & 269 & 250 & 249 & 250 \\
144 & 259 & 250 & 252 & 250 \\
175 & 262 & 250 & 251 & 250 \\
$\mathbf{3 6 3}$ & $\mathbf{2 4 1}$ & $\mathbf{2 5 0}$ & $\mathbf{2 5 1}$ & $\mathbf{2 5 0}$ \\
\hline
\end{tabular}

Table B19 - AB-B003

\begin{tabular}{|c|cccc|}
\hline $\begin{array}{c}\text { Time } \\
\text { (min) }\end{array}$ & $\begin{array}{c}\text { Furnace T: Act } \\
\text { I SP }\left({ }^{\circ} \mathbf{C}\right)\end{array}$ & \multicolumn{2}{|c|}{$\begin{array}{c}\text { Process T: } \\
\text { Act } / \text { SP }\left({ }^{\circ} \mathbf{C}\right)\end{array}$} \\
\hline 0 & 162 & 350 & 36 & 250 \\
15 & 353 & 350 & 155 & 250 \\
20 & 346 & 250 & 180 & 250 \\
$\mathbf{4 5}$ & $\mathbf{2 4 9}$ & $\mathbf{2 6 0}$ & $\mathbf{2 4 5}$ & $\mathbf{2 5 0}$ \\
150 & 237 & 250 & 254 & 250 \\
260 & 232 & 250 & 251 & 250 \\
300 & 246 & 250 & 251 & 250 \\
$\mathbf{3 4 5}$ & $\mathbf{2 2 2}$ & $\mathbf{2 5 0}$ & $\mathbf{2 4 8}$ & $\mathbf{2 5 0}$ \\
\hline
\end{tabular}


Table B20 - Air Blown Digest Analysis Data

\begin{tabular}{|c|c|c|c|c|c|c|c|c|c|c|c|}
\hline \multirow{2}{*}{$\begin{array}{l}\text { Sample } \\
\text { Name }\end{array}$} & \multicolumn{2}{|c|}{$\%$ Ash } & \multicolumn{2}{|c|}{$\begin{array}{l}\text { \% Conradson } \\
\text { Carbon Yield }\end{array}$} & \multicolumn{2}{|c|}{$\begin{array}{c}\% \text { WVU } \\
\text { Coke Yield }\end{array}$} & \multicolumn{2}{|c|}{$\begin{array}{l}\text { Softening } \\
\text { Point }\left({ }^{\circ} \mathrm{C}\right)\end{array}$} & \multicolumn{2}{|c|}{$\begin{array}{c}\text { Toluene } \\
\text { Insoluble (\%) }\end{array}$} & \multirow[t]{2}{*}{$\begin{array}{c}\text { Density } \\
\text { (g/cc) }\end{array}$} \\
\hline & 1 & 2 & 1 & 2 & 1 & 2 & 1 & 2 & 1 & 2 & \\
\hline AB-A086 & 1.17 & 1.19 & 34.56 & 35.05 & 37.22 & 36.03 & 194.1 & 98.0 & 37.43 & 91 & 1.2547 \\
\hline 90 & 50 & 0.51 & 36.81 & 38.31 & 40.89 & 40.43 & 143.5 & 144.3 & 37.21 & 53 & 1.2345 \\
\hline AB-A095 & .06 & 0.06 & 39.88 & 39.06 & 42.74 & 41.74 & 134.2 & 134.4 & 33.38 & 33.51 & 1.2038 \\
\hline B-A098 & 2.72 & 2.49 & 37.93 & 36.58 & 41.04 & 39.91 & 191.7 & 207.9 & 38.62 & 38.67 & 1.2700 \\
\hline$\overline{B-A}$ & 1.64 & 2.00 & 37.98 & 37.53 & 44.80 & 38.52 & 142.9 & 143.5 & 35.78 & 36.06 & 1.2181 \\
\hline $\mathrm{AB}-\mathrm{B} 003$ & 0.65 & 0.65 & 35.14 & 34.61 & 36.34 & 36.12 & 164.0 & 163.1 & 35.09 & 35.26 & 1.2286 \\
\hline
\end{tabular}

Table B21 - Vacuum Distilled Digest Analysis Data

\begin{tabular}{|c|cc|cc|}
\hline \multirow{2}{*}{$\begin{array}{c}\text { Sample } \\
\text { Name }\end{array}$} & \multicolumn{2}{|c|}{ \% Ash } & \multicolumn{2}{c|}{$\begin{array}{c}\text { \%VU Coke } \\
\text { Yield }\end{array}$} \\
\cline { 2 - 5 } & $\mathbf{1}$ & $\mathbf{2}$ & $\mathbf{1}$ & $\mathbf{2}$ \\
\hline VD-A086 & 115.9 & 116.3 & 45.80 & 46.50 \\
\hline VD-A090 & 80.9 & 77.4 & 40.30 & 41.60 \\
\hline VD-A095 & 170.5 & 175.5 & 60.80 & 62.40 \\
\hline VD-A098 & 152.2 & 155.3 & 57.34 & 58.09 \\
\hline VD-A100 & 112.8 & 113.1 & 48.39 & 47.77 \\
\hline VD-B003 & 105.8 & 105.6 & 47.18 & 46.52 \\
\hline
\end{tabular}


Table B22 - FTIR Raw Data

\begin{tabular}{|c|c|c|c|c|}
\hline & \multicolumn{4}{|c|}{ Peak Areas } \\
\hline Sample Name & (Har + Hal) & Har & $\mathrm{Hal}$ & $(\mathrm{C}=\mathrm{C}) \mathrm{ar}$ \\
\hline Raw CBB & 124.276 & 73.765 & 50.511 & 30.712 \\
\hline Raw CBB baselined & 124.404 & 73.521 & 50.883 & 31.028 \\
\hline HCBB-L1 & $13 \overline{3} .092$ & 75.111 & 57.981 & 32.689 \\
\hline HCBB-L1 baselined & 132.398 & 74.881 & 57.517 & 32.67 \\
\hline HCBB-L2 & 129.276 & 70.705 & 58.571 & 29.222 \\
\hline HCBB-L2 baselined & 129.313 & 70.717 & 58.595 & $3 \overline{1.724}$ \\
\hline HCBB-L3 & 145.886 & 74.913 & 70.974 & 31.729 \\
\hline HCBB-L3 baselined & 147.078 & 75.648 & 71.43 & 31.864 \\
\hline Raw MO & 200.773 & 56.744 & 144.029 & 34.191 \\
\hline Raw MO baselined & 202.167 & 57.539 & 144.629 & 34.186 \\
\hline HMO-L3 & 206.695 & 51.114 & 155.581 & $30.6 \overline{9}$ \\
\hline HMO-L3 baselined & 206.298 & 50.984 & 155.313 & 30.63 \\
\hline Raw SO & 385.936 & 23.386 & 362.55 & 20.995 \\
\hline Raw SO baselined & 386.258 & 23.373 & 362.885 & 20.988 \\
\hline HSO-L3 & 323.564 & 28.178 & 295.386 & 23.179 \\
\hline HSO-L3 baselined & 323.24 & 28.086 & 295.154 & 23.169 \\
\hline A086 & 148.474 & 70.577 & $7 \overline{89} \overline{7}$ & 55.216 \\
\hline A086 baselined & 148.531 & 70.598 & $7 \overline{9} . \overline{3}$ & 55.221 \\
\hline A090 & 19.446 & $5 . \overline{5} 53$ & 14.093 & $5.6 \overline{1}$ \\
\hline A090 baselined & 19.417 & 5.342 & 14.075 & 5.609 \\
\hline A095 & $10 \overline{2} .052$ & 17.543 & 84.509 & 13.376 \\
\hline A095 baselined & $10 \overline{2} .118$ & 17.563 & 84.555 & 13.378 \\
\hline A098 & 6.047 & 3.417 & 2.629 & 2.979 \\
\hline A098 baselined & $\overline{6} . \overline{0} 2 \overline{2}$ & 3.408 & 2.615 & $2.977^{--}$ \\
\hline$\overline{A 100}$ & 1799.0̄7̄̄ & 602.66 & 1196.416 & 293.978 \\
\hline A100 baselined & 1803.115 & 604.031 & $119 \overline{9} .085$ & 294.105 \\
\hline B003 & 177.762 & 73.363 & 104.399 & 46.545 \\
\hline B003 baselined & $17 \overline{6} 6$ & 73.325 & 104.336 & 46.543 \\
\hline AB-A086 & 44.343 & 26.028 & 18.315 & $13.40 \overline{1}$ \\
\hline AB-A086 baselined & 43.471 & 25.63 & 17.841 & 13.383 \\
\hline AB-A090 & 30.356 & 11.107 & 19.25 & 9.078 \\
\hline AB-A090 baselined & 30.283 & 11.075 & 19.208 & 9.082 \\
\hline AB-A095 & 25.71 & 4.408 & 21.302 & 5.1 \\
\hline AB-A095 baselined & 25.67 & 4.393 & 21.277 & 5.099 \\
\hline AB-A098 & 8.559 & 5.719 & 2.84 & 2.291 \\
\hline AB-A098 baselined & 8.539 & 5.695 & $2 . \overline{4} \overline{4}$ & 2.291 \\
\hline AB-A100 & 39.213 & 13.92 & 25.293 & 7.513 \\
\hline AB-A100 baselined & 39.108 & 13.881 & 25.228 & $7.512^{-}$ \\
\hline AB-B003 & 15.663 & 7.741 & $7 . \overline{9} 23$ & 2.471 \\
\hline AB-B003 baselined & 15.396 & 7.627 & 7.769 & 2.47 \\
\hline Half HCBB-L3, Half HSO-L3 & $2 \overline{4} 6 . \overline{5} \overline{1}$ & 195.198 & 51.253 & $32.34 \overline{7}$ \\
\hline Half HCBB-L3, Half HSO-L3 baselined & 246.165 & 195.04 & 51.124 & 32.335 \\
\hline Half HCBB-L3, Half HMO-L3 & 198.001 & 130.222 & 67.779 & 36.306 \\
\hline
\end{tabular}


Table B23 - Elemental Analysis Raw Data

\begin{tabular}{|c|c|c|c|c|c|c|c|c|}
\hline Sample & $\% \mathrm{C}$ & $\begin{array}{l}\text { Std. } \\
\text { Dev. }\end{array}$ & $\% \mathrm{H}$ & $\begin{array}{l}\text { Std. } \\
\text { Dev. }\end{array}$ & $\% \mathrm{~N}$ & Std. Dev. & $\% \mathrm{~s}$ & $\begin{array}{l}\text { Std. } \\
\text { Dev. }\end{array}$ \\
\hline$\overline{\mathrm{CBB}}$ & 91.5764 & 0.28078 & 7119 & 0.00100 & 1.0866 & 0.02493 & 0.4763 & 0.01659 \\
\hline HCBB-L1 & .1533 & 6332 & 5.8110 & 01011 & 9719 & & 0.4249 & 51445 \\
\hline HCBB-L2 & 91.9730 & 08767 & 5.8233 & 0.02338 & 1.0383 & 0.06200 & 0.2983 & 0.03476 \\
\hline HCBB-L3 & 91 & 10807 & 5.8430 & 0.00985 & 0.9642 & 236 & 0.1625 & 0.00143 \\
\hline SO & & 8531 & & 04882 & 0.44 & & 36 & \\
\hline HSO-L3 & 87.1264 & 23346 & 8.9831 & 0.08379 & 0.5128 & 0.04904 & 2.3765 & 0.07350 \\
\hline$\overline{0}$ & $=-$ & 0.45136 & 7.7 & 0.12248 & 0.3694 & 0.0 & 0.9635 & 0.01237 \\
\hline MO-L3 & .1922 & 30244 & 7.8201 & 0.04267 & 0.3977 & 0.1 & 0.7539 & 0.03369 \\
\hline$\overline{0}$ & $92.4070^{-}$ & 47034 & 6.2072 & 0.05101 & 0.9417 & 579 & 0.2737 & 0.15040 \\
\hline$\overline{086}$ & 90.6363 & 22276 & 5.9363 & 0.01059 & 1.5661 & 0.0 & 0.3760 & 0.03112 \\
\hline 90 & 9.67 & 81714 & 6.6656 & 0.07779 & 1.3458 & 0.1 & 1.1881 & 0.02215 \\
\hline 4095 & 89.0003 & 0.30883 & 7.6051 & 0.10792 & 0.7455 & 0.05343 & 2.1811 & 0.07719 \\
\hline$\overline{A 098}$ & $90.2744^{-}$ & 0.57445 & 5.8655 & 0.05439 & 1.1984 & 0.0 & 0.3844 & 0.02807 \\
\hline 100 & & 5186 & & 0.0 & 0.5665 & & & 0.05843 \\
\hline$\overline{B 003}$ & 90.6945 & 0.16566 & 6.4126 & 0.00742 & 0.9041 & 0.02872 & 0.5559 & 0.04222 \\
\hline \begin{tabular}{|l|}
$A B-A 086$ \\
\end{tabular} & 90.1747 & 0.26776 & 5.1950 & 0.02840 & 1.6260 & 0.03041 & 0.4260 & 0.02044 \\
\hline$\overline{A B-A 090}$ & 90. & 0.21800 & 5.5945 & 0.01490 & 1.4410 & & 1.3325 & 0.01114 \\
\hline$\overline{A B-A 095}$ & & & & & 0.84 & & & - הono \\
\hline AB-A098 & 89.9293 & 0.13703 & 4.9918 & 0.00986 & 1.2566 & 0.02468 & 0.4202 & 0.00089 \\
\hline B-A10 & 91.4437 & 0.75151 & 5.9006 & 0.04223 & 0.8065 & 0.02576 & 1.0752 & 0.01259 \\
\hline B-B00 & 90.8184 & 0.34928 & 5.4825 & 0.03844 & 1.0044 & 0.02997 & 0.7139 & 0.01411 \\
\hline
\end{tabular}




\section{Table B24 - Simulated Distillation Boiling Point Curve Data}

\begin{tabular}{|c|c|c|c|c|c|c|c|c|}
\hline$\%$ Off & $\overline{C B B}$ & SO & MO & HCBB-L1 & HCBB-L2 & HCBB-L3 & HMO-L3 & HSO-L3 \\
\hline by Vol & $\mathrm{BP}(\mathrm{C})$ & $\mathrm{BP}(\mathrm{C})$ & $\mathrm{BP}(\mathrm{C})$ & $\mathrm{BP}(\mathrm{C})$ & $\mathrm{BP}(\mathrm{C})$ & $\mathrm{BP}(\mathrm{C})$ & $\mathrm{BP}(\mathrm{C})$ & $\mathrm{BP}(\mathrm{C})$ \\
\hline 0 & 234.7 & 161.7 & 136.5 & 235.0 & 23.4 & 22.2 & 140.7 & 102.1 \\
\hline 1 & 236.0 & 167.2 & 139.3 & 236.0 & 238.3 & 22.6 & 158.8 & 132.1 \\
\hline 2 & 236.9 & 191.6 & 161.7 & 236.7 & 238.4 & 23.7 & 162.7 & 138.9 \\
\hline 3 & 237.3 & 211.0 & 164.5 & 237.1 & 238.5 & 261.7 & 164.8 & 159.8 \\
\hline 4 & 237.7 & 230.2 & 166.5 & 237.4 & 238.6 & 261.9 & 166.8 & 164.1 \\
\hline 5 & 238.0 & 249.3 & 168.7 & 237.7 & 238.6 & 262.1 & 170.0 & 170.6 \\
\hline 6 & 238.3 & 262.1 & 172.5 & 237.9 & 238.7 & 262.4 & 181.3 & 184.5 \\
\hline 7 & 238.6 & 275.3 & 185.2 & 238.1 & 238.8 & 262.7 & 186.3 & 190.6 \\
\hline 8 & 238.8 & 284.4 & 189.1 & 238.3 & 238.8 & 263.0 & 190.1 & 197.7 \\
\hline 9 & 239.0 & 289.0 & 192.6 & 238.5 & 238.9 & 263.5 & 193.2 & 204.7 \\
\hline 10 & 239.2 & 296.9 & 195.9 & 238.7 & 239.0 & 264.3 & 196.5 & 212.5 \\
\hline 11 & 239.4 & 303.5 & 198.7 & 238.8 & 239.1 & 265.0 & 199.4 & 222.6 \\
\hline 12 & 239.5 & 308.6 & 201.7 & 239.0 & 239.1 & 287.2 & 202.9 & 228.0 \\
\hline 13 & 239.7 & 312.4 & 205.6 & 239.1 & 239.2 & 287.3 & 206.9 & 233.4 \\
\hline 14 & 239.9 & 317.1 & 209.4 & 239.3 & 239.3 & 287.5 & 210.4 & 237.2 \\
\hline 15 & 240.0 & 320.9 & 213.5 & 239.4 & 239.3 & 287.6 & 214.2 & 243.1 \\
\hline 16 & 240.2 & 324.4 & 218.0 & 239.5 & 239.4 & 287.7 & 219.9 & 249.4 \\
\hline 17 & 240.3 & 329.2 & 224.7 & 239.7 & 239.5 & 287.8 & 224.8 & 254.4 \\
\hline 18 & 240.5 & 334.3 & 227.6 & 239.8 & 239.6 & 287.8 & 226.8 & 259.9 \\
\hline 19 & 240.6 & 338.2 & 229.7 & 239.9 & 239.9 & 287.9 & 228.6 & 261.5 \\
\hline 20 & 240.7 & 341.2 & 231.7 & 240.0 & 240.1 & 287.9 & 230.5 & 262.8 \\
\hline 21 & 240.8 & 343.7 & 234.2 & 240.1 & 240.3 & 288.0 & 233.0 & 265.6 \\
\hline 22 & 241.0 & 346.4 & 237.6 & 240.2 & 245.2 & 288.1 & 235.4 & 271.2 \\
\hline 23 & 241.1 & 349.3 & 239.7 & 240.3 & 250.2 & 288.1 & 237.7 & 276.0 \\
\hline 24 & 241.2 & 352.9 & 241.0 & 240.5 & 250.6 & 288.2 & 239.0 & 280.7 \\
\hline 25 & 241.4 & 356.4 & 242.0 & 240.6 & 251.3 & 288.3 & 239.9 & 283.8 \\
\hline 26 & 241.6 & 359.5 & 242.9 & 240.8 & 256.9 & 288.3 & 240.7 & 285.7 \\
\hline 27 & 241.8 & 361.9 & 243.9 & 240.9 & 260.7 & 288.4 & 241.5 & 287.9 \\
\hline 28 & 242.0 & 364.6 & 246.1 & 241.1 & 261.3 & 288.5 & 244.0 & 289.5 \\
\hline 29 & 242.3 & 367.1 & 250.7 & 241.4 & 261.8 & 288.5 & 247.1 & 293.6 \\
\hline 30 & 243.0 & 370.3 & 253.3 & 245.1 & 262.3 & 288.5 & 249.5 & 295.9 \\
\hline 31 & 250.1 & 372.5 & 255.3 & 250.6 & 262.8 & 288.6 & 251.6 & 299.0 \\
\hline 32 & 251.4 & 375.1 & 257.1 & 251.4 & 263.4 & 288.6 & 253.2 & 303.0 \\
\hline 33 & 252.4 & 377.8 & 259.1 & 252.4 & 264.6 & 288.6 & 254.9 & 307.1 \\
\hline 34 & 253.8 & 380.6 & 261.7 & 256.7 & 270.6 & 288.7 & 256.7 & 309.7 \\
\hline 35 & 260.2 & 383.4 & 264.0 & 260.8 & 275.6 & 288.7 & 258.9 & 312.3 \\
\hline 36 & 261.9 & 386.2 & 265.3 & 261.9 & 286.8 & 288.8 & 260.7 & 315.9 \\
\hline 37 & 262.7 & 389.0 & 266.4 & 262.6 & 287.4 & 288.8 & 262.6 & 318.1 \\
\hline 38 & 263.4 & 391.5 & 267.3 & 263.3 & 287.7 & 288.8 & 263.9 & 320.7 \\
\hline 39 & 264.1 & 393.9 & 268.0 & 263.9 & 288.0 & 288.9 & 264.8 & 322.8 \\
\hline 40 & 265.2 & 396.2 & 268.7 & 265.2 & 288.2 & 288.9 & 265.5 & 326.9 \\
\hline
\end{tabular}


Table B24 - Simulated Distillation Boiling Point Curve Data (cont.)

\begin{tabular}{|c|c|c|c|c|c|c|c|c|}
\hline$\%$ Off & CBB & SO & MO & HCBB-L1 & HCBB-L2 & HCBB-L3 & HMO-L3 & HSO-L3 \\
\hline by Vol & $\mathrm{BP}(\mathrm{C})$ & $\mathrm{BP}(\mathrm{C})$ & $\mathrm{BP}(\mathrm{C})$ & $\mathrm{BP}(\mathrm{C})$ & $\mathrm{BP}(\mathrm{C})$ & $\mathrm{BP}(\mathrm{C})$ & $\mathrm{BP}(\mathrm{C})$ & $\mathrm{BP}(\mathrm{C})$ \\
\hline 41 & 266.7 & 399.1 & 269.4 & 269.6 & 288.4 & 289.0 & 266.1 & 331.6 \\
\hline 42 & 276.2 & 401.7 & 269.9 & 276.1 & 288.6 & 289.0 & 266.6 & 335.7 \\
\hline 43 & 285.7 & 404.6 & 270.5 & 286.8 & 288.7 & 289.0 & 267.1 & 338.8 \\
\hline 44 & 288.1 & 407.5 & 271.1 & 287.9 & 288.9 & 289.1 & 267.6 & 341.2 \\
\hline 45 & 288.7 & 410.2 & 271.7 & 288.5 & 289.0 & 289.1 & 268.0 & 343.5 \\
\hline 46 & 289.2 & 412.9 & 272.4 & 288.9 & 289.2 & 289.1 & 268.6 & 346.2 \\
\hline 47 & 289.6 & 415.5 & 273.2 & 289.2 & 289.3 & 289.2 & 269.5 & 349.4 \\
\hline 48 & 289.9 & 418.5 & 274.1 & 289.5 & 289.4 & 289.2 & 270.4 & 352.7 \\
\hline 49 & 290.2 & 421.4 & 276.4 & 289.7 & 289.5 & 289.3 & 271.2 & 356.1 \\
\hline 50 & 290.4 & 424.2 & 278.7 & 290.0 & 289.6 & 289.3 & 272.6 & 359.1 \\
\hline 51 & 290.7 & 427.2 & 281.0 & 290.2 & 289.7 & 289.3 & 274.8 & 361.5 \\
\hline 52 & 290.9 & 430.3 & 282.8 & 290.4 & 289.8 & 289.4 & 276.5 & 364.4 \\
\hline 53 & 291.1 & 433.4 & 284.7 & 290.5 & 289.9 & 289.5 & 278.7 & 367.5 \\
\hline 54 & 291.3 & 436.5 & 286.8 & 290.7 & 290.0 & 289.5 & 280.3 & 370.8 \\
\hline 55 & 291.5 & 439.5 & 288.6 & 2909 & 290.1 & 289.6 & 282.1 & 373.1 \\
\hline 56 & 291.7 & 442.5 & 289.8 & 291.0 & 290.2 & 289.7 & 284.2 & 376.2 \\
\hline 57 & 291.9 & 445.8 & 290.9 & 291.2 & 290.3 & 289.7 & 286.1 & 379.2 \\
\hline 58 & 292.1 & 449.3 & 291.8 & 291.4 & 290.5 & 289.8 & 287.4 & 382.3 \\
\hline 59 & 292.4 & 452.5 & 292.7 & 291.5 & 290.7 & 289.9 & 288.4 & 385.6 \\
\hline 60 & 292.6 & 455.8 & 293.5 & 291.7 & 290.9 & 289.9 & 289.2 & 388.8 \\
\hline 61 & 294.6 & 459.2 & 294.4 & 291.9 & 292.9 & 290.0 & 289.9 & 391.7 \\
\hline 62 & 296.3 & 462.8 & 295.3 & 294.8 & 294.8 & 290.1 & 290.7 & 394.4 \\
\hline 63 & 296.8 & 466.4 & 296.1 & 295.9 & 295.3 & 290.1 & 291.5 & 397.6 \\
\hline 64 & 297.3 & 470. & 2968 & 296.4 & 295.5 & 294.4 & 292.4 & 400.8 \\
\hline 65 & 297.6 & 475.1 & 297.6 & 296.8 & 295.8 & 294.6 & 293.1 & 404.2 \\
\hline 66 & 297.9 & 479.7 & 298.3 & 297.1 & 296.0 & 294.7 & 293.8 & 407.7 \\
\hline 67 & 298.2 & 484.3 & 299.1 & 297.3 & 296.2 & 294.8 & 294.5 & 411.0 \\
\hline 68 & 298.4 & 489.0 & 300.7 & 297.6 & 296.3 & 294.9 & 295.2 & 414.3 \\
\hline 69 & 298.7 & 493.8 & 302.6 & 297.8 & 296.5 & 295.0 & 296.1 & 418.0 \\
\hline 70 & 298.9 & 497.8 & 303.9 & 298.0 & 296.6 & 295.2 & 297.9 & 421.6 \\
\hline 71 & 299.2 & 500.9 & 304.8 & 298.2 & 296.7 & 295.3 & 299.8 & 425.2 \\
\hline 72 & 299.5 & 504.2 & 305.7 & 298.4 & 296.9 & 295.3 & 300.9 & 429.2 \\
\hline 73 & 3002 & 507.6 & 306.7 & 298.6 & 297.0 & 295.4 & 301.7 & 433.3 \\
\hline 74 & 301.0 & 510.9 & 3084 & 299.3 & 297.2 & 295.5 & 302.5 & 437.3 \\
\hline 75 & 301.8 & 514.4 & 310.4 & 300.1 & 297.4 & 295.5 & 303.7 & 441.2 \\
\hline 76 & 303.1 & 5180 & 312.3 & 3009 & 297.6 & 295.6 & 305.7 & 445.7 \\
\hline 77 & 305.7 & 521.6 & 314.5 & 302.0 & 297.8 & 295.6 & 307.8 & 450.3 \\
\hline 78 & 310.3 & 524.7 & 316.0 & 305.0 & 298.6 & 295.7 & 309.9 & 454.7 \\
\hline 79 & 313.8 & 527.6 & 317.6 & 310.0 & 299.2 & 295.7 & 312.0 & 459.6 \\
\hline 80 & 315.2 & 530.6 & 318.9 & 313.5 & 299.8 & 295.8 & 313.4 & 464.5 \\
\hline
\end{tabular}


Table B24 - Simulated Distillation Boiling Point Curve Data (cont.)

\begin{tabular}{|c|c|c|c|c|c|c|c|c|}
\hline$\%$ Off & CBB & SO & MO & HCBB-L1 & HCBB-L2 & HCBB-L3 & HMO-L3 & HSO-L3 \\
\hline by Vol & $\mathrm{BP}(\mathrm{C})$ & $\mathrm{BP}(\mathrm{C})$ & $\mathrm{BP}(\mathrm{C})$ & $\mathrm{BP}(\mathrm{C})$ & $\mathrm{BP}(\mathrm{C})$ & $\mathrm{BP}(\mathrm{C})$ & $\mathrm{BP}(\mathrm{C})$ & $\mathrm{BP}(\mathrm{C})$ \\
\hline 81 & 316.9 & 533.6 & 320.5 & 314.7 & 300.6 & 295.8 & 314.8 & 470.2 \\
\hline 82 & 318.5 & 536.8 & 322.6 & 316.3 & 301.7 & 295.9 & 316.1 & 476.8 \\
\hline 83 & 319.3 & 540.0 & 324.7 & 317.9 & 304.9 & 296.0 & 317.6 & 483.3 \\
\hline 84 & 321.2 & 543.4 & 326.1 & 318.8 & 309.9 & 296.0 & 319.9 & 490.4 \\
\hline 85 & 323.4 & 546.8 & 327.3 & 320.4 & 313.2 & 296.1 & 322.0 & 497.1 \\
\hline 86 & 333.6 & 550.5 & 328.8 & 322.5 & 313.9 & 296.1 & 323.2 & 502.0 \\
\hline 87 & 339.6 & 554.2 & 330.3 & 333.0 & 315.0 & 296.2 & 324.2 & 507.1 \\
\hline 88 & 342.2 & 558.1 & 331.6 & 338.8 & 316.9 & 296.3 & 326.0 & 512.4 \\
\hline 89 & 347.0 & 562.2 & 334.2 & 341.4 & 317.5 & 296.3 & 327.3 & 518.2 \\
\hline 90 & 351.5 & 566.5 & 338.9 & 346.4 & 318.3 & 296.4 & 329.1 & 523.7 \\
\hline 91 & 352.3 & 571.1 & 342.3 & 351.1 & 320.0 & 296.5 & 333.0 & 528.5 \\
\hline 92 & 352.9 & 576.0 & 345.2 & 351.8 & 321.9 & 296.6 & 337.9 & 533.7 \\
\hline 93 & 353.4 & 581.3 & 347.6 & 352.4 & 338.3 & 296.7 & 340.9 & 539.2 \\
\hline 94 & 353.9 & 587.0 & 349.7 & 352.9 & 340.5 & 296.8 & 343.6 & 545.2 \\
\hline 95 & 355.1 & 593.4 & 353.2 & 353.5 & 346.6 & 296.9 & 346.1 & 551.8 \\
\hline 96 & 359.0 & 600.6 & 358.6 & 354.4 & 350.6 & 296.9 & 349.0 & 559.2 \\
\hline 97 & 369.9 & 609.1 & 364.0 & 358.2 & 351.0 & 297.0 & 354.5 & 567.6 \\
\hline 98 & 376.1 & 619.6 & 371.7 & 370.6 & 351.4 & 297.9 & 360.9 & 577.9 \\
\hline 99 & 400.2 & 633.9 & 379.5 & 394.5 & 351.8 & 298.2 & 369.2 & 591.5 \\
\hline 100 & 402.4 & 644.2 & 390.3 & 401.8 & 352.0 & 298.4 & 373.9 & 601.2 \\
\hline
\end{tabular}

Table B25 Viscosity Data for A086

\begin{tabular}{|c|c|c|}
\hline $\mathrm{T}\left({ }^{\circ} \mathrm{C}\right)$ & RPM & Viscosity (cP) \\
\hline \multirow[t]{5}{*}{50} & 12.5 & 3359.28 \\
\hline & 13.5 & 3337.07 \\
\hline & 14.5 & 3326.19 \\
\hline & 15.5 & 3320.58 \\
\hline & 16.5 & 3304.75 \\
\hline \multirow[t]{5}{*}{60} & 46 & 923.28 \\
\hline & 49 & 916.95 \\
\hline & 52 & 911.34 \\
\hline & 55 & 901.99 \\
\hline & 58 & 900.84 \\
\hline
\end{tabular}


Table B26 - Viscosity Data for A090

\begin{tabular}{|c|c|c|}
\hline T ( $\left.{ }^{\circ} \mathrm{C}\right)$ & RPM & Viscosity (cP) \\
\hline 50 & 18 & 2032.90 \\
\hdashline & 20 & 2006.57 \\
\hline & 22 & 1982.30 \\
\hline & 24 & 1972.08 \\
\hline & 26 & 1965.73 \\
\hline 60 & 48 & 812.33 \\
\hline & 51 & 783.36 \\
\hline-54 & 770.95 \\
\hline & 57 & 757.73 \\
\hline & 60 & 750.84 \\
\hline
\end{tabular}

Table B27 - Viscosity Data for A095

\begin{tabular}{|c|c|c|}
\hline $\mathrm{T}\left({ }^{\circ} \mathrm{C}\right)$ & RPM & Viscosity (cP) \\
\hline \multirow[t]{5}{*}{50} & 3.7 & 111913.51 \\
\hline & 4.0 & 106560.00 \\
\hline & 4.3 & 100613.95 \\
\hline & 4.6 & 97808.70 \\
\hline & 4.9 & 96000.00 \\
\hline \multirow[t]{5}{*}{60} & 13 & 34363.08 \\
\hline & 14 & 33782.86 \\
\hline & 15 & 33237.33 \\
\hline & 16 & 32720.00 \\
\hline & 17 & 32376.47 \\
\hline \multirow[t]{5}{*}{70} & 30 & 14848.00 \\
\hline & 32 & 14580.00 \\
\hline & 34 & 14211.76 \\
\hline & 36 & 14008.89 \\
\hline & 38 & 13827.37 \\
\hline \multirow[t]{5}{*}{80} & 70 & 6619.43 \\
\hline & 75 & 6536.53 \\
\hline & 80 & 6448.00 \\
\hline & 85 & 6339.76 \\
\hline & 90 & 6272.00 \\
\hline \multirow[t]{5}{*}{90} & 120 & 3534.77 \\
\hline & 130 & 3574.15 \\
\hline & 140 & 3492.57 \\
\hline & 150 & 3434.67 \\
\hline & $160^{-}$ & 3376.00 \\
\hline \multirow[t]{5}{*}{100} & 210 & 2081.52 \\
\hline & 220 & 2056.73 \\
\hline & 230 & 2031.30 \\
\hline & 240 & 2000.00 \\
\hline & 250 & 1986.56 \\
\hline 110 & 250 & 1438.72 \\
\hline 120 & 250 & 1088.00 \\
\hline 130 & 250 & 837.12 \\
\hline
\end{tabular}


Table B28 - Viscosity Data for A098

\begin{tabular}{|c|c|c|}
\hline $\mathrm{T}\left({ }^{\circ} \mathrm{C}\right)$ & RPM & Viscosity (cP) \\
\hline \multirow[t]{5}{*}{50} & 2.4 & 15371.72 \\
\hline & 2.7 & 15063.45 \\
\hline & 3.0 & 14816.84 \\
\hline & 3.3 & 14687.78 \\
\hline & 3.6 & 14546.90 \\
\hline \multirow[t]{5}{*}{60} & 8 & 4536.53 \\
\hline & 9 & 4319.08 \\
\hline & 10 & 4181.11 \\
\hline & 11 & 4079.13 \\
\hline & 12 & 4014.14 \\
\hline \multirow[t]{5}{*}{70} & 27 & 1475.24 \\
\hline & 29 & 1414.87 \\
\hline & 31 & 1373.90 \\
\hline & 33 & 1354.26 \\
\hline & 35 & 1336.86 \\
\hline \multirow[t]{5}{*}{80} & 65 & 609.10 \\
\hline & 70 & 584.45 \\
\hline & 75 & 563.88 \\
\hline & 80 & 554.88 \\
\hline & 85 & 546.24 \\
\hline
\end{tabular}

Table B29 - Viscosity Data for A100

\begin{tabular}{|c|c|c|}
\hline $\mathbf{T}\left({ }^{\circ} \mathbf{C}\right)$ & RPM & Viscosity (cP) \\
\hline 40 & 130.0 & 296.71 \\
\hdashline & 140.0 & 293.94 \\
\hdashline & 150.0 & 289.54 \\
\hdashline & 160.0 & 290.94 \\
\hline
\end{tabular}

Table B30 - Viscosity Data for B003

\begin{tabular}{|c|c|c|}
\hline $\mathrm{T}\left({ }^{\circ} \mathrm{C}\right)$ & RPM & Viscosity (cP) \\
\hline \multirow[t]{5}{*}{50} & 26.0 & 1407.39 \\
\hline & 29.0 & 1327.99 \\
\hline & 32.0 & 1278.48 \\
\hline & 35.0 & 1246.02 \\
\hline & 38.0 & 1228.16 \\
\hline \multirow[t]{5}{*}{60} & 90 & 485.90 \\
\hline & 95 & 481.16 \\
\hline & 100 & 478.10 \\
\hline & 105 & 475.90 \\
\hline & 110 & 473.90 \\
\hline
\end{tabular}


Table B31 - Viscosity Data for AB-A086

\begin{tabular}{|c|c|c|}
\hline $\mathrm{T}\left({ }^{\circ} \mathrm{C}\right)$ & RPM & Viscosity (cP) \\
\hline 210 & 0.1 & 506891.84 \\
\hline \multirow{2}{*}{220} & 0.2 & 136170.94 \\
\hline & 0.3 & 135970.99 \\
\hline \multirow[t]{5}{*}{230} & 0.4 & 74084.19 \\
\hline & 0.5 & 70185.02 \\
\hline & 0.6 & 65785.96 \\
\hline & 0.7 & 65043.26 \\
\hline & 0.8 & 66360.84 \\
\hline \multirow[t]{5}{*}{240} & 4.7 & 9738.35 \\
\hline & 4.9 & 9451.04 \\
\hline & 5.1 & 9339.18 \\
\hline & 5.3 & 9224.45 \\
\hline & 5.5 & 9128.96 \\
\hline \multirow[t]{5}{*}{250} & 10 & 4607.02 \\
\hline & 10.5 & 4559.03 \\
\hline & 11 & 4569.93 \\
\hline & 11.5 & 4632.06 \\
\hline & 12 & 4624.01 \\
\hline \multirow[t]{5}{*}{260} & 20 & 2288.51 \\
\hline & 21 & 2313.79 \\
\hline & 22 & 2260.43 \\
\hline & 23 & 2274.3 \\
\hline & 24 & 2247.02 \\
\hline \multirow[t]{5}{*}{270} & 42 & 1112.62 \\
\hline & 44 & 1120.67 \\
\hline & 46 & 1112.37 \\
\hline & 48 & 1117.26 \\
\hline & 50 & 1121.76 \\
\hline \multirow[t]{5}{*}{280} & 69 & 659.86 \\
\hline & 72 & 673.19 \\
\hline & 75 & 668.66 \\
\hline & 78 & 671.4 \\
\hline & 81 & 679.11 \\
\hline
\end{tabular}


Table B32 - Viscosity Data for AB-A090

\begin{tabular}{|c|c|c|}
\hline $\mathrm{T}\left({ }^{\circ} \mathrm{C}\right)$ & RPM & Viscosity (cP) \\
\hline \multirow[t]{2}{*}{170} & 0.1 & 3020800 \\
\hline & $0 . \overline{2}$ & 2886400 \\
\hline \multirow[t]{4}{*}{180} & 0.3 & 913066.67 \\
\hline & 0.4 & 868800 \\
\hline & 0.5 & 853760 \\
\hline & $0 . \overline{6}$ & 849066.67 \\
\hline \multirow[t]{5}{*}{190} & 1.1 & 357818.18 \\
\hline & 1.2 & 344533.33 \\
\hline & 1.3 & 334276.92 \\
\hline & 1.4 & 325942.86 \\
\hline & 1.5 & 320000 \\
\hline \multirow[t]{5}{*}{200} & 4 & 115040 \\
\hline & 4.2 & 111390.48 \\
\hline & 4.4 & 106909.09 \\
\hline & 4.6 & 106017.39 \\
\hline & 4.8 & 104666.67 \\
\hline \multirow[t]{5}{*}{210} & 9.5 & 48370.53 \\
\hline & 10 & 47936 \\
\hline & 10.5 & 47603.81 \\
\hline & 11 & 47360 \\
\hline & 11.5 & 47304.35 \\
\hline \multirow[t]{5}{*}{220} & 21 & 22643.81 \\
\hline & 22 & 22778.18 \\
\hline & 23 & 23067.83 \\
\hline & 24 & 23146.67 \\
\hline & 25 & 23270.4 \\
\hline \multirow[t]{5}{*}{230} & 35 & 13165.71 \\
\hline & 37 & 13267.03 \\
\hline & 39 & 13193.84 \\
\hline & 41 & 13065.37 \\
\hline & 43 & 12963.72 \\
\hline \multirow[t]{5}{*}{240} & 60 & 7349.33 \\
\hline & 65 & 7453.54 \\
\hline & 70 & 7433.14 \\
\hline & 75 & 7424 \\
\hline & 80 & 7424 \\
\hline \multirow[t]{5}{*}{250} & 90 & 4323.56 \\
\hline & 100 & 4294.4 \\
\hline & 110 & 4305.45 \\
\hline & 120 & 4277.33 \\
\hline & 130 & 4243.69 \\
\hline \multirow[t]{5}{*}{260} & 150 & 2666.67 \\
\hline & 160 & 2636 \\
\hline & 170 & 2627.76 \\
\hline & 180 & 2624 \\
\hline & 190 & 2627.37 \\
\hline \multirow[t]{5}{*}{270} & 210 & 1749.33 \\
\hline & 220 & 1748.36 \\
\hline & 230 & 1769.74 \\
\hline & 240 & 1784 \\
\hline & 250 & 1809.92 \\
\hline 280 & 250 & 1392.64 \\
\hline
\end{tabular}


Table B33 - Viscosity Data for AB-A095

\begin{tabular}{|c|c|c|}
\hline$\overline{\mathrm{T}\left({ }^{\circ} \mathrm{C}\right)}$ & RPM & Viscosity (cP) \\
\hline 150 & 0.1 & 283139.58 \\
\hline 150 & 0.2 & 240848.61 \\
\hline 160 & 0.3 & 89180.97 \\
\hline 160 & 0.4 & 81732.56 \\
\hline 160 & 0.5 & 79543.03 \\
\hline 160 & 0.6 & 74484.11 \\
\hline 160 & 0.7 & 73098.69 \\
\hline 170 & 1.5 & 23754.93 \\
\hline 170 & 1.7 & 23395.01 \\
\hline 170 & 1.9 & 22858.28 \\
\hline 170 & 2.1 & 22252.39 \\
\hline 170 & 2.3 & 21908.37 \\
\hline 180 & 4.5 & 8318.23 \\
\hline 180 & 5 & 8158.26 \\
\hline 180 & 5.5 & 7994.66 \\
\hline 180 & 6 & 7898.31 \\
\hline 180 & 6.5 & 7835.25 \\
\hline 190 & 10 & 3731.2 \\
\hline 190 & 11 & 3664.67 \\
\hline 190 & 12 & 3639.22 \\
\hline 190 & 13 & 3608.46 \\
\hline 190 & 14 & 3582.09 \\
\hline 200 & 22 & 1796.89 \\
\hline 200 & 24 & 1782.12 \\
\hline 200 & 26 & 1783.47 \\
\hline 200 & 28 & 1769.62 \\
\hline 200 & 30 & 1763.62 \\
\hline 210 & 43 & 991.88 \\
\hline 210 & 46 & 993.7 \\
\hline 210 & 49 & 998.97 \\
\hline 210 & 52 & 1000.17 \\
\hline 210 & 55 & 983.79 \\
\hline
\end{tabular}


Table B34 - Viscosity Data for AB-A098

\begin{tabular}{|c|c|c|}
\hline $\mathrm{T}\left({ }^{\circ} \mathrm{C}\right)$ & RPM & Viscosity (cP) \\
\hline 210 & 0.2 & 188059.87 \\
\hline \multirow[t]{5}{*}{220} & 0.5 & 55668.12 \\
\hline & 0.6 & 54588.35 \\
\hline & 0.7 & 53474.3 \\
\hline & 0.8 & 51813.94 \\
\hline & 0.9 & 50989.12 \\
\hline \multirow[t]{5}{*}{230} & 2.6 & 14512.29 \\
\hline & 2.8 & 14268.38 \\
\hline & 3 & 14037 \\
\hline & 3.2 & 13984.52 \\
\hline & 3.4 & 13955.85 \\
\hline \multirow[t]{5}{*}{240} & 6.8 & 6016.36 \\
\hline & 7.3 & 5949.42 \\
\hline & 7.8 & 5944.89 \\
\hline & 8.3 & 5926.45 \\
\hline & 8.8 & 5964.64 \\
\hline \multirow[t]{5}{*}{250} & 14 & 2866.53 \\
\hline & 15 & 2811.4 \\
\hline & 16 & 2774.41 \\
\hline & 17 & 2773.53 \\
\hline & 18 & 2719.42 \\
\hline \multirow[t]{5}{*}{260} & 28 & 1411.84 \\
\hline & 30 & 1403.7 \\
\hline & 32 & 1379.71 \\
\hline & 34 & 1386.76 \\
\hline & 36 & 1423.03 \\
\hline \multirow[t]{5}{*}{270} & 50 & 749.84 \\
\hline & 55 & 744.93 \\
\hline & 60 & 726.84 \\
\hline & 65 & 732.77 \\
\hline & 70 & 727.56 \\
\hline
\end{tabular}


Table B35 - Viscosity Data for AB-A100

\begin{tabular}{|c|c|c|}
\hline $\mathrm{T}\left({ }^{\circ} \mathrm{C}\right)$ & RPM & Viscosity (cP) \\
\hline \multirow[t]{2}{*}{160} & 0.1 & 313133.18 \\
\hline & 0.2 & 297236.58 \\
\hline \multirow[t]{3}{*}{170} & 0.3 & 102778.07 \\
\hline & 0.4 & 97779.14 \\
\hline & 0.5 & 96339.44 \\
\hline \multirow[t]{5}{*}{180} & 1 & 36892.13 \\
\hline & 1.1 & 35446.98 \\
\hline & 1.2 & 34292.68 \\
\hline & 1.3 & 33223.68 \\
\hline & 1.4 & 32693.02 \\
\hline \multirow[t]{5}{*}{190} & 3.2 & 11772.49 \\
\hline & 3.4 & 11309.35 \\
\hline & 3.6 & 11030.98 \\
\hline & 3.8 & 10955.56 \\
\hline & 4 & 10977.66 \\
\hline \multirow[t]{5}{*}{200} & 9 & 4832.3 \\
\hline & 9.5 & 4824.23 \\
\hline & 10 & 4816.97 \\
\hline & 10.5 & 4816.12 \\
\hline & 11 & 4809.88 \\
\hline \multirow[t]{5}{*}{210} & 18 & 2436.15 \\
\hline & 19 & 2440.53 \\
\hline & 20 & 2411.49 \\
\hline & 21 & 2410.91 \\
\hline & 22 & 2410.39 \\
\hline \multirow[t]{5}{*}{220} & 34 & 1261.5 \\
\hline & 36 & 1246.4 \\
\hline & 38 & 1220.27 \\
\hline & 40 & 1210.24 \\
\hline & 42 & 1199.74 \\
\hline \multirow[t]{5}{*}{230} & 55 & 699.12 \\
\hline & 60 & 697.85 \\
\hline & 65 & 693.08 \\
\hline & 70 & 693.28 \\
\hline & 75 & 691.85 \\
\hline
\end{tabular}


Table B36 - Viscosity Data for AB-B003

\begin{tabular}{|c|c|c|}
\hline $\mathrm{T}\left({ }^{\circ} \mathrm{C}\right)$ & RPM & Viscosity (cP) \\
\hline 195 & 0.1 & 4224000 \\
\hline \multirow[t]{5}{*}{205} & 0.5 & 652800 \\
\hline & 0.6 & 626133.33 \\
\hline & 0.7 & 606171.43 \\
\hline & 0.8 & 592800 \\
\hline & 0.9 & 580266.67 \\
\hline \multirow[t]{5}{*}{215} & 1.8 & 194133.33 \\
\hline & 2 & 188160 \\
\hline & 2.2 & 183563.64 \\
\hline & 2.4 & 180000 \\
\hline & 2.6 & 173538.46 \\
\hline \multirow[t]{5}{*}{225} & 7.5 & 58965.33 \\
\hline & 8 & 58960 \\
\hline & 8.5 & 58202.35 \\
\hline & 9 & 57386.67 \\
\hline & 9.5 & 56656.84 \\
\hline \multirow[t]{5}{*}{235} & 15 & 27690.67 \\
\hline & 16 & 26600 \\
\hline & 17 & 26164.71 \\
\hline & 18 & 25706.67 \\
\hline & 19 & 25229.47 \\
\hline \multirow[t]{5}{*}{245} & 36 & 12444.44 \\
\hline & 38 & 11991.58 \\
\hline & 40 & 11696 \\
\hline & 42 & 11489.52 \\
\hline & $44^{-}$ & 11170.91 \\
\hline \multirow[t]{5}{*}{255} & 75 & 5811.2 \\
\hline & 80 & 5648 \\
\hline & 85 & 5481.41 \\
\hline & 90 & 5361.78 \\
\hline & $95^{-}$ & 5301.89 \\
\hline \multirow[t]{5}{*}{265} & 145 & 3014.62 \\
\hline & 155 & 3026.58 \\
\hline & 165 & 2932.36 \\
\hline & 175 & 2907.43 \\
\hline & 185 & 2902.49 \\
\hline \multirow[t]{5}{*}{275} & 210 & 1895.62 \\
\hline & 220 & 1847.27 \\
\hline & 230 & 1897.74 \\
\hline & 240 & 1853.33 \\
\hline & 250 & 1871.36 \\
\hline 285 & 250 & 1369.6 \\
\hline 295 & 250 & 998.4 \\
\hline
\end{tabular}




\section{APPENDIX C: $\quad$ MATERIAL SAFETY DATA SHEETS}

(abbreviated) 


\section{Material Safety Data Sheet}

MATERIAL K O P P E R S

SAFETY

DATA

SHEET

KOPPERS INC.

436 SEVENTH AVENUE

PITTSBURGH， PA. 15219-1800
MEDICAL EMERGENCIES: 1800 553-5631

OUTSIDE U.S.A.: $\quad 412$ 227-2001

GENERAL INFORMATION: 412 227-2424

CHEMTREC ASSISTANCE $1800 \quad 424-9300$

CANUTEC: $\quad 1613996-6666$

\section{SECTION I - PRODUCT IDENTIFICATION}

PRODUCT NAME: Carbon Black Base \#1

SYNONYM: None

PRODUCT USE: Burned to produce carbon black.

CHEMICAL FAMILY: Coal tar distillate

FORMULA: Complex mixture of hydrocarbons

CAS NUMBER: 65996-92-1

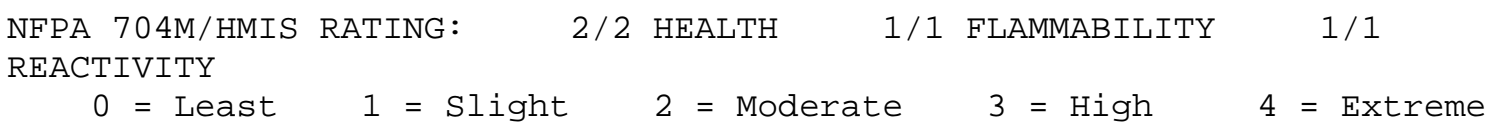

CANADIAN PRODUCT CLASSIFICATION: Class D, Division 2, Subdivision A, Very Toxic Material

\section{SECTION II - HEALTH/SAFETY ALERT WARNING}

MAY BE FATAL IF SWALLOWED

HARMFUL TO THE SKIN OR IF INHALED

CAUSES EYE AND SKIN IRRITATION

AVOID PROLONGED OR REPEATED CONTACT

OBSERVE GOOD HYGIENE AND SAFETY PRACTICES WHEN HANDLING THIS

PRODUCT DO NOT USE THIS PRODUCT UNTIL MSDS HAS BEEN READ AND UNDERSTOOD WARNING: THIS PRODUCT CONTAINS A CHEMICAL KNOWN TO THE STATE OF CALIFORNIA TO CAUSE CANCER. 


\section{$* * * *$ MATERIAL SAFETY DATA SHEET}

1, 2, 3, 4-Tetrahydronaphthalene 23050

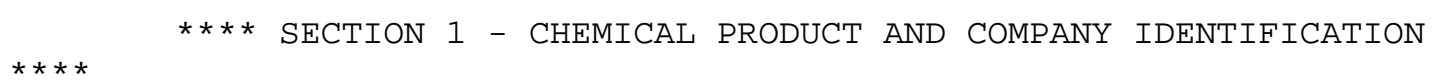




\section{Material Safety Data Sheet}

Tetrahydrofuran (uninhibited)

\section{Section 1 - Chemical Product and Company I dentification}

MSDS Name: Tetrahydrofuran (uninhibited)

Synonyms: Diethylene oxide; 1,4-Epoxybutane; Tetramethylene oxide; THF;

Oxacyclopentane.

Company I dentification: Fisher Scientific

1 Reagent Lane

Fair Lawn, NJ 07410

For information, call: 201-796-7100

Emergency Number: 201-796-7100

For CHEMTREC assistance, call: 800-424-9300

For International CHEMTREC assistance, call: 703-527-3887

Section 2 - Composition, Information on Ingredients

\begin{tabular}{|c|l|c|c|}
\hline CAS\# & Chemical Name & Percent & EI NECS/ ELI NCS \\
\hline $109-99-9$ & Tetrahydrofuran & 100 & $203-726-8$ \\
\hline
\end{tabular}

Hazard Symbols: XI F

Risk Phrases: 1119 36/37

\section{Section 3 - Hazards Identification}

\section{EMERGENCY OVERVIEW}

Appearance: colorless liquid. Flash Point: -14 deg C. Danger! May cause central nervous system depression. Uninhibited material, or material from which the inhibitor has been removed or reacted, may form explosive peroxides. May cause liver and kidney damage. May cause lung damage. May be harmful if swallowed. Hygroscopic (absorbs moisture from the air). Extremely flammable liquid and vapor. Vapor may cause flash fire. Causes severe eye irritation and possible eye injury. Causes skin and respiratory tract irritation. May be absorbed through intact skin.

Target Organs: Kidneys, central nervous system, liver, lungs, respiratory system, eyes, skin.

\section{Potential Health Effects}

Eye: Contact with eyes may cause severe irritation, and possible eye burns. Vapors may cause eye irritation. Damage may be permanent.

Skin: Causes skin irritation. May be absorbed through the skin. If absorbed, causes symptoms similar to those of inhalation. THF is not a skin sensitizer in animals.

I ngestion: May cause gastrointestinal irritation with nausea, vomiting and diarrhea. May cause central nervous system depression. May be harmful if swallowed.

I nhalation: Inhalation of high concentrations may cause central nervous system effects characterized by nausea, headache, dizziness, unconsciousness and coma. Vapors may cause dizziness or suffocation. Inhalation may cause coughing, difficulty breathing and loss of consciousness. Causes irritation of the mucous membrane and upper respiratory tract. Inhalation of tetrahydrofuran vapors may cause abnormal liver function as detected by laboratory tests. (Dupont) 


\section{Material Safety Data Sheet}

Toluene

Section 1 - Chemical Product and Company Identification

MSDS Name: Toluene

Synonyms: Methacide; Methylbenzene; Methylbenzol; Phenylmethane; Toluol.

Company I dentification: Fisher Scientific

1 Reagent Lane

Fair Lawn, NJ 07410

For information, call: 201-796-7100

Emergency Number: 201-796-7100

For CHEMTREC assistance, call: $800-424-9300$

For International CHEMTREC assistance, call: 703-527-3887

\begin{tabular}{|c|c|c|c|}
\hline \multicolumn{4}{|c|}{ Section 2 - Composition, Information on Ingredients } \\
\hline CAS\# & Chemical Name & Percent & EI NECS/ ELI NCS \\
\hline $108-88-3$ & Toluene & $>99$ & $203-625-9$ \\
\hline
\end{tabular}

Hazard Symbols: XN F

Risk Phrases: 1120

\section{Section 3 - Hazards I dentification}

\section{EMERGENCY OVERVIEW}

Appearance: colorless liquid. Flash Point: 40 deg F. Flammable liquid and vapor. May cause central nervous system depression. May cause liver and kidney damage. This substance has caused adverse reproductive and fetal effects in animals. Causes digestive and respiratory tract irritation. May cause skin irritation. Aspiration hazard if swallowed. Can enter lungs and cause damage. Danger! Harmful or fatal if swallowed. Causes eye irritation and possible transient injury. Poison! May be absorbed through intact skin. Vapor harmful. Call physician immmediately.

Target Organs: Kidneys, central nervous system, liver.

Potential Health Effects

Eye: Causes eye irritation. May result in corneal injury. Vapors may cause eye irritation.

Skin: May cause skin irritation. Prolonged and/or repeated contact may cause irritation and/or dermatitis. May be absorbed through the skin.

Ingestion: Aspiration hazard. May cause irritation of the digestive tract. May cause effects similar to those for inhalation exposure. Aspiration of material into the lungs may cause chemical pneumonitis, which may be fatal.

I nhalation: Inhalation of high concentrations may cause central nervous system effects characterized by nausea, headache, dizziness, unconsciousness and coma. Inhalation of vapor may cause respiratory tract irritation. May cause liver and kidney damage. Vapors may cause dizziness or suffocation. Overexposure may cause dizziness, tremors, restlessness, rapid heart beat, increased blood pressure, hallucinations, acidosis, kidney failure. 


\section{Material Safety Data Sheet}

ACC\# 96038

Methyl sulfoxide, $99.7 \%$

Section 1 - Chemical Product and Company Identification

MSDS Name: Methyl sulfoxide, 99.7\%

Catalog Numbers: AC127790000, AC127790010, AC127790025, AC127790200, AC127790250, AC127790500 AC127790500, AC127791000

Synonyms: Methyl sulfoxide; DMSO; Sulfinylbis(methane); Dimethyl sulfoxide.

Company I dentification:

Acros Organics N.V.

One Reagent Lane

Fair Lawn, NJ 07410

For information in North America, call: 800-ACROS-01

For emergencies in the US, call CHEMTREC: 800-424-9300

\begin{tabular}{|c|l|c|c|}
\hline \multicolumn{4}{|c|}{ Section 2 - Composition, Information on Ingredients } \\
\hline CAS\# & Chemical Name & Percent & EI NECS/ ELI NCS \\
\hline $67-68-5$ & Dimethyl sulfoxide & 99.7 & $200-664-3$ \\
\hline
\end{tabular}

Hazard Symbols: XI

Risk Phrases: 36/37/38

\section{Section 3 - Hazards Identification}

\section{EMERGENCY OVERVIEW}

Appearance: clear liquid. May be absorbed through intact skin. Hygroscopic (absorbs moisture from the air). May cause liver and kidney damage. Caution! Causes eye and skin irritation. Causes respiratory tract irritation.

Target Organs: Kidneys, liver, eyes, skin, mucous membranes.

Potential Health Effects

Eye: Produces irritation, characterized by a burning sensation, redness, tearing, inflammation, and possible corneal injury. May cause chemical conjunctivitis.

Skin: May cause irritation with burning pain, itching and redness. Substance is rapidly absorbed through the skin.

Ingestion: May cause gastrointestinal irritation with nausea, vomiting and diarrhea. May cause liver and kidney damage. May cause garlic smell on the breath and body. I nhalation: May cause respiratory tract irritation. Can produce delayed pulmonary edema.

Chronic: Prolonged or repeated skin contact may cause dermatitis. May cause liver and kidney damage. Effects may be delayed. 


\title{
**** MATERIAL SAFETY DATA SHEET
}

\author{
Carbon disulfide $\quad 04280$
}

$* * *$ SECTION 1 - CHEMICAL PRODUCT AND COMPANY IDENTIFICATION $* * * *$

MSDS Name: Carbon disulfide

Catalog Numbers: C/2880, C/2920

Synonyms: Carbon bisulfide; Dithiocarbonic anhydride; Sulphocarbonic

anhydride.

Company Identification: Fisher Scientific UK

Bishop Meadow Road

Loughborough, Leicestershire

LE11 5RG, UK

For information, call: 01509231166

For emergencies, call: 01509231166

*** * SECTION 2 - COMPOSITION, INFORMATION ON INGREDIENTS ***

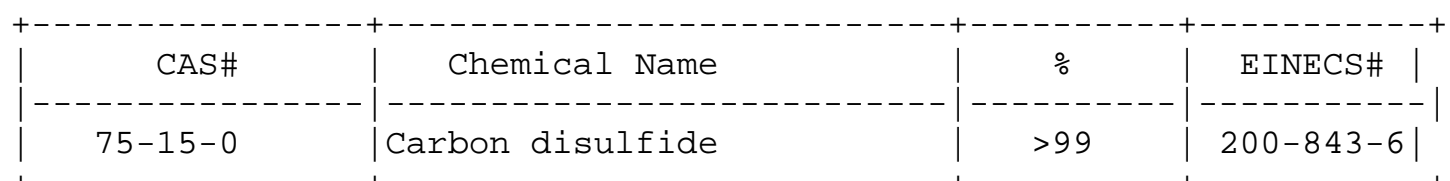

Hazard Symbols: T F $\quad$ Risk Phrases: $1136 / 38$ 48/23 6263

*** SECTION 3 - HAZARDS IDENTIFICATION ****

EMERGENCY OVERVIEW

Highly flammable. Irritating to eyes and skin. Toxic : danger of serious damage to health by prolonged exposure through inhalation. Possible risk of impaired fertility. Possible risk of harm to the unborn child. Stench.

Potential Health Effects

Eye: May cause severe eye irritation.

Skin: Causes skin irritation. May be absorbed through the skin in harmful amounts. Prolonged and/or repeated contact may cause defatting of the skin and dermatitis. Dermatitis and vesiculation may result from skin contact with the vapor or liquid.

Ingestion: May cause digestive tract disturbances. May cause effects similar to those for inhalation exposure. Aspiration of material into the lungs may cause chemical pneumonitis, which may be fatal. Can cause nervous system damage. Ingestion may cause convulsions, seizures and possible coma.

Inhalation: Intoxication can involve all parts of the central and peripheral nervous systems including damage to the nerves with paresthesias, muscle weakness, unsteady gait, and tremors. Exposure may accelerate the development or worsen, coronary heart disease.

Chronic: Prolonged or repeated exposure can cause psychic abnormalities such as anxiety, depression and excitability. May cause reproductive and fetal effects. Chronic exposure may cause visual disturbances. Repeated exposure may cause central and peripheral nervous system damage and digestive tract disturbances. Chronic exposure may cause coronary heart disease. 
MSDS ID NO.: 0161MAR019

\section{Material Safety Data Sheet}

Revision date: 02/05/2004

\section{IDENTIFICATION OF THE SUBSTANCE/PREPARATION AND} THECOMPANY/UNDERTAKING

Product name: MAPLLC Carbonblack Feedstock

Synonyms: Catalytic Cracked Clarified Oil; Catalytic Cracked Slurry Oil; Slurry Oil

Chemical Family: Petroleum Hydrocarbon

Formula: Mixture

Supplier:

Marathon Ashland Petroleum LLC

539 SOUTH MAIN STREET

FINDLAY OH 45840

Other information: 419-421-3070

Emergency telephone number: 877-627-5463

\section{COMPOSITION/INFORMATION ON INGREDIENTS}

Carbonblack Feedstock is a complex mixture of hydrocarbons produced as the residual fraction of distillation products from a catalytic cracking process. It consists of hydrocarbons having carbon numbers predominantly $>\mathrm{C} 20$ and boiling above $662 \mathrm{~F}$. The CAS description of this stream states that it is likely to contain $>5 \% 4$ to 6 -membered condensed ring polycyclic aromatic hydrocarbons. This product was analyzed by MAP and found to contain $1.2-2.3 \%$ of the 22 3-7 ring polycyclic aromatic compounds identified as Persistent Bioaccumulative Toxic (PBT) chemicals subject to reporting under EPA EPCRA Section 313 regulations.

\section{Product information}

Name CAS Number Weight \% ACGIH Exposure

Limits: OSHA - Vacated

PELs - Time Weighted Ave

Other: MAPLLC Carbonblack Feedstock 64741-62-4 100

\section{Component Information}

Product name: MAPLLC Carbonblack Feedstock Page 1 of 12 MSDS ID NO.: 0161MAR019

Name CAS Number Weight \% ACGIH Exposure

Limits: OSHA - Vacated

PELs - Time Weighted Ave

Other: Cat. Cracked Clarified Oil 64741-62-4 100

Sulfur Compounds Mixture 001.0000 - 003.0000

5-methylchrysene 3697-24-3 000.1000 - 000.6000

Benzo(j)fluoranthene 205-82-3 000.0900 - 000.5000

Benzo(a)phenanthrene 218-01-9 000.1000 - 000.3000 $=0.2 \mathrm{mg} / \mathrm{m}^{3}$

TWA as benzene soluble aerosol $=0.2 \mathrm{mg} / \mathrm{m}^{3}$

TWA benzene soluble fraction

Benzo(a)pyrene 50-32-8 000.0500 $-000.1000=0.2 \mathrm{mg} / \mathrm{m}^{3}$ TWA as benzene soluble aerosol

$=0.2 \mathrm{mg} / \mathrm{m}^{3}$ TWA benzene soluble fraction

Hydrogen Sulfide 7783-06-4 $<000.0100=10$ ppm TWA

$=15 \mathrm{ppm}$ STEL

$=10 \mathrm{ppm}$ TWA

$=14 \mathrm{mg} / \mathrm{m}^{3}$ TWA

$=15 \mathrm{ppm}$ STEL

$=21 \mathrm{mg} / \mathrm{m}^{3} \mathrm{STEL}$

Notes: The manufacturer has voluntarily elected to reflect exposure limits contained in OSHA's 1989 air contaminants standard in its MSDS's, even though certain of those exposure limits were vacated in 1992. 
MSDS ID NO.: 0229MAR019

\section{Material Safety Data Sheet}

Revision date: 02/02/2004

1. IDENTIFICATION OF THE SUBSTANCE/PREPARATION AND THECOMPANY/UNDERTAKING

Product name: MAPLLC Pitch Vacuum Overheads

Synonyms: Distillate, Heavy Thermal Cracked; Dubbs Vacuum Overhead; Maraflex 1000; Pitch

Vac Oh; PVO Oil

Chemical Family: Petroleum Pitch

Formula: Mixture

Supplier:

Marathon Ashland Petroleum LLC

539 SOUTH MAIN STREET

FINDLAY OH 45840

Other information: 419-421-3070

Emergency telephone number: 877-627-5463

\section{COMPOSITION/INFORMATION ON INGREDIENTS}

Pitch Vacuum Overheads (PVO) is a complex mixture of hydrocarbons produced as distillate from a thermal cracking process. It consists predominantly of unsaturated hydrocarbons having carbon numbers predominantly in the range of $\mathrm{C} 15-\mathrm{C} 36$ and boiling in the range of 500 to $896 \mathrm{~F}$. The CAS description of this stream states that it is likely to contain $>5 \% 4$ to 6 -membered condensed ring polycyclic aromatic hydrocarbons. This product was analyzed by MAP and found to contain $0.8 \%$ of the 22 3-7 ring polycyclic aromatic compounds identified as Persistent Bioaccumulative Toxic (PBT) Chemicals subject to reporting under EPA EPCRA Section 313 regulations.

\section{Product information}

Name CAS Number Weight \% ACGIH Exposure

Limits:

OSHA - Vacated

PELs - Time

Weighted Ave

Other:

MAPLLC Pitch Vacuum Overheads 64741-81-7 100

\section{Component Information}

Name CAS Number Weight \% ACGIH Exposure

Limits:

OSHA - Vacated

PELs - Time

Weighted Ave

Other:

Distillates (Petroleum), Heavy

Thermal Cracked

64741-81-7 100

Benzo(j,k)fluorene 206-44-0 000.1000 - 000.3000

Sulfur Compounds Mixture 000.5000 - 004.0000

Benzo(a)phenanthrene 218-01-9 000.1000 $-000.2000=0.2 \mathrm{mg} / \mathrm{m}^{3}$ TWA

as benzene soluble

aerosol

$=0.2 \mathrm{mg} / \mathrm{m}^{3}$ TWA

benzene soluble

fraction

Benz(a)anthracene 56-55-3 000.1000 - 000.1500

Product name: MAPLLC Pitch Vacuum

Overheads

Page 1 of 10 MSDS ID NO.: 0229MAR019

Notes: The manufacturer has voluntarily elected to reflect exposure limits contained in OSHA's 1989 air contaminants standard in its MSDS's, even though certain of those exposure limits were vacated in 1992.

Product name: MAPLLC Pitch Vacuum

Overheads 